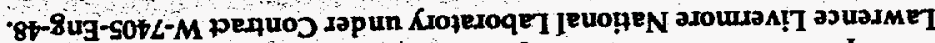

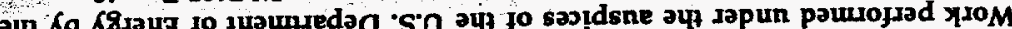
KjopesoqeT गut jo asout aq jou Kew do

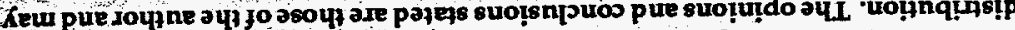

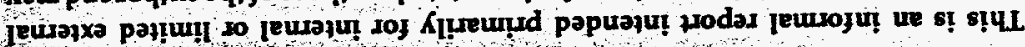

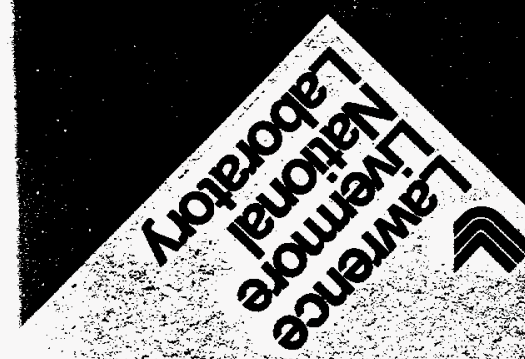
1.

\title{
966I.I KrensqaA
}

\section{If ueuplaseH つ’T}

$$
\text { nM uวg }
$$

sianos $0 \%$

\section{S66I TINT

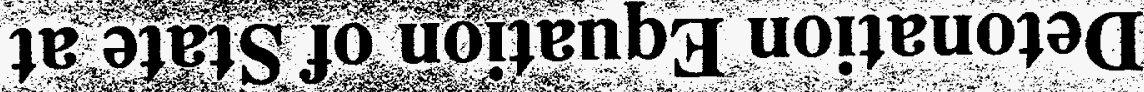

1150

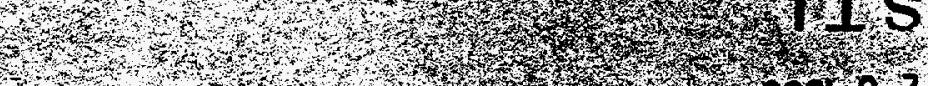

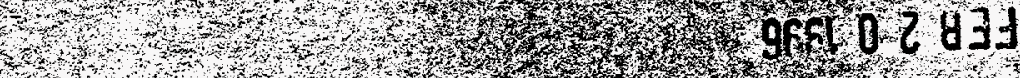

.

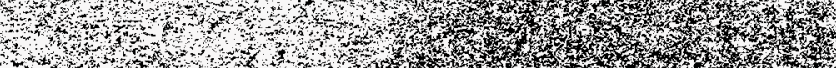

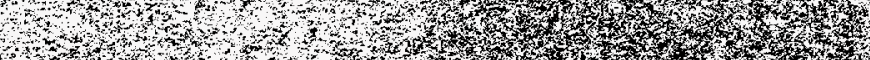

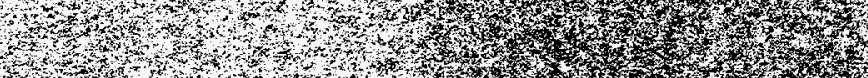

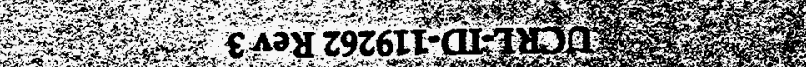

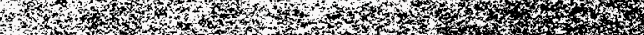


Th is document was prepared as an account of work sponsored by an agency of the United States Government. Neither the United States Government nor the University of California nor any of their employ es, makes any warranty, express or implied, or assumes any legal fiability or responsibility for the accuracy, completeness, or usefulness of any information, a pparatus, product, or process disclosed, or represents that its use would not infringe privately owned rights. Reference herein to any specific commercial products, process, or service by trade name, trademark, manufacturer, or otherwise, does not necessarily constitute or imply its endorsement. recommendation, of favoring by the United States Government or the Universit F of Calfornia. The Views and opinions of authors expressed herein do not necessarily state or reflect those of the United States Government or the University of Californiz, and shall not be used for advertising or product endorsenent purposes.

The University of California, and shall not be used for ad vertising o

This report has been reproduced directly from the best available copy

Available to $\mathrm{DOE}$ and DOE contractors from the Office of Scientific and Technical Information

P.O. Box 62, Oak Ridge, TN 37831

Prices available from (615) 576-8401, FTS 626-8401

Available to the public from the

National Tectnical Information Service

U.S. Department of Commerce

5285 Port Royal Rd.

Springfièl, VA 22161 


\title{
Detonation Equation of State at LLNL, 1995
}

\author{
P. C. Souers, Ben Wu and L. C. Haselman, Jr.
}

with Contributions from Larry Fried, Raul Garza, Dave Goosman, Don Griswold, Frank Helm, Neil Holmes, Ron Lee, Stella McGuire, Al Nichols, W. J. Pitz, Francis Ree, Craig Tarver, Matt van Thiel, Harry Vantine and Choong-Shik Yoo.

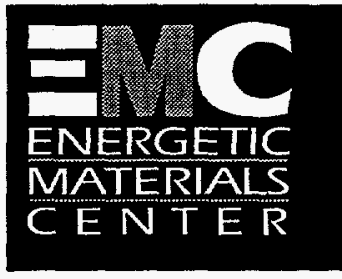

UCRL-ID-119262 Rev. 3

Energetic Materials Center

Lawrence Livermore National Laboratory

Livermore, CA 94566

February 1, 1996 


\begin{abstract}
JWL's and 1-D Look-up tables are shown to work for "one-track" experiments like cylinder tests, plate shots and the expanding sphere. They fail for "many-track" experiments like the compressed sphere. As long as the one-track experiment has dimensions larger than the explosive's reaction zone and the explosive is near-ideal, a general JWL with $R_{1}=4.5$ and $R_{2}=1.5$ can be constructed, with both $\omega$ and $\mathrm{E}_{\mathrm{o}}$ being calculated from thermochemical codes. These general JWL's allow comparison between various explosives plus recalculation of the JWL for different densities. The Bigplate experiment complements the cylinder test by providing continuous oblique angles of shock incidence from $0^{\circ}$ to $70^{\circ}$. Explosive reaction zone lengths are determined from metal plate thicknesses, extrapolated run-to-detonation distances, radius size effects and detonation front curvature. Simple theories of the cylinder test, Bigplate, the cylinder size effect and detonation front curvature are given. The detonation front lag at the cylinder edge is shown to be proportional to the half-power of the reaction zone length. By calibrating for wall blow-out, a full set of reaction zone lengths from PETN to ANFO are obtained. The $1800-2100 \mathrm{~K}$ freezing effect is shown to be caused by rapid cooling of the product gases. Compiled comparative data for about 80 explosives is listed. Ten Chapters plus an Appendix.
\end{abstract}




\section{DISCLAMMER}

Portions of this document may be illegible in electronic image products. Images are produced from the best available original document. 


\section{Table of Contents}

Chapter 1. Introduction

1. C-J Theory

2. Energy Partition in C-J Theory

3. ZND Theory and the Existence of the C-J Point

4. Definition of the JWL

5. Adiabatic and Gruneison Gamma

6. The One-Dimensional Table

7. Program Burn/ Beta Burn Package

8. Terminilogy of Detonation Points

9. Units, etc.

Chapter 2. JWL Construction

1. Cylinder JWL's: the Old Method

2. Observations from Data

3. The Freeze Assumption

4. The Reasons why CHEQ is Good at Large Volumes (by Al Nichols)

5. Setting $\omega$ from $\mathrm{CHEQ} /$ What determines $\omega$

6. Infinite-Volume Energy in the JWL

7. Fixing $\mathrm{R}_{1}$

8. JWL-Changing with Density

Chapter 3. Cylinder JWL Fits

1. The Code and JWL-Making

2. JWL Cylinder Results (with Stella McGuire)

3. The Detonation Energy Standard

4. Problem: Changing the Density of LX-14

5. Problem: Calculating an HMX Derivitive

Chapter 4. More Cylinders

1. Comparing Metal-Pushing

2. Using CHEETAH to Generate JWL's (with Larry Fried)

3. Fabry dependence on the Velocity Angle

4. Accuracy of the Cylinder Fabry Trace (with Dave Goosman)

5. Accuracy of the Cylinder Streak Camera (with Raul Garza and Frank Helm)

6. Simple Theory of the Cylinder

7. Jump-Off Velocities for the Cylinder and the Plate

Chapter 5. Bigplate

1. Bigplate: a Oblique Angle EOS Test

2. Simple Model of Oblique Contact with a Wall

3. Detailed Code Studies

4. Angular Behavior in the Code

5. EOS Comparison in the Code

6. Bigplate as an EOS Test 
Chapter 6. Plates and the Expanding Sphere

1. Electric Gun Flatness

2. Small Metal Plate Shots and the Reaction Zone

3. Effect of the Hugoniot on a Plate Shot

4. The Expanding Wilkins' Spheres

Chapter 7. Imploding Spheres

1. Compression and the Hugoniot

2. Reacted Hugoniot Data from Supracompression

3. Hugoniot EOS's for LX-14 and Others

4. The French Compressed PBX-9404 Sphere

5. Measuring High Pressure Adiabats

6. Calculating High Pressure Adiabats for LX-14 (with Al Nichols)

Chapter 8. Size Effects

1. Reaction Zone Definitions

2. Size Effect on Detonation Velocity

3. Calculating the Inverse Radius Model

4. Detonation Front Curvature Fits

5. Reaction-Zone Model of the Curvature

6. Estimate of the Shadow Velocity

Chapter 9. More Reaction Zones

1. Time Constant on the Prinicpal Rayleigh Line

2. Walker-Wasley and Reaction Zones

3. Run-to-Detonation Distance

4. Reaction Zones in Ignition \& Growth (with Craig Tarver)

5. Simplest I\&G Rate Term that causes Curvature (with Craig Tarver)

Chapter 10. Temperature and Thermochemical Codes

1. Direct Temperature Measurements (by Choong-Shik Yoo and Neil Holmes)

2. Semi-Empirical Calibration of the CHEQ Intermolecular Potentials (by Matt van Thiel and Francis Ree)

3. Carbon not the Reason for the Freeze (by Francis Ree, W. J. Pitz, and Matt van Thiel)

4. Temperature Quenching is the Reason for the Freeze (by Francis Ree, W. J. Pitz, and Matt van Thiel)

5. Variability of the Freeze?

Appendix A: Explosives and their Properties

Appendix B: Impedance Calculations

1. Simple Flyer into Explosive

2. Simple Forward Detonation into a Plate

3. Improved Flyer to Explosive Problem

4. Defining the Instant of Impact 


\section{Chapter 1. Introduction}

An Equation of State (EOS) is the description of explosive performance that is used as the input to a hydrodynamic code (hydrocode), a Newtonian $\mathrm{F}=$ ma engine running on a computer. The EOS can come from a test or a thermochemical code, a semi-empirical computer program that can predict a chemical reaction using thermodynamics. The thermochemical code and the hydrocode live in different worlds, with different points of view and different code-runners.

The first report sought to collect old explosives data and show its several-percent crudity relative to complex models with fraction-of-a-percent precision. 1 This report deals with "one track" EOS models and how well they do in hydrocodes. A one-track EOS is an input with the P-v path laid out explicitly and it is usually, but not always, the Principal Adiabat. It can be a JWL, a many-term polynomial, a time-dependent combination of JWL's or a 1-D table. Such an EOS will work well for any one-track experiment, which includes cylinders, flat plates and expanding spheres. It does not work reproduceably for any geometry with many tracks, eg. a compressed sphere. A complex geometry probably cannot be described by a single track.

\section{C-J Theory}

This turn-of-the century theory is illustrated in Fig. 1-1a. The explosive detonates instantly and completely at the C-J point, which always occurs for a given explosive at the same pressure, $P$, and relative volume, $v$, It lies on the Principal Rayleigh Line, which is determined from combined mass and momentum conservation :

$$
P=\rho_{0} D^{2}(1-v)
$$

where $\rho_{o}$ is initial explosive density, and $D$ is the detonation velocity . One end of this line is anchored on the C-J point and the other at the starting room condition where $\mathrm{v}=1$. Eq. 1 may also be rewritten in terms of particle (ie. mass) velocity $u_{p}$ as

$$
P=\rho_{o} D u_{p}
$$

Thermochemical codes are built around C-J Theory. Such a code starts at room conditions, then dumps in all of the energy of detonation, $E_{0}$. The code then guesses the reaction products of the 
explosive and calcuates their thermodynamic properties. This causes a big jump in pressure and temperature at $\mathrm{v}=1$. The code then compresses the reaction products with maximum entropy increase, a line called the reacted Hugoniot. At the same time, it calculates possible Rayleigh Lines. Each point on the Hugoniot is the composition with minimum Gibbs free energy. The intersection of the Hugoniot and the Rayleigh Line that are tangent to each other is taken as the C-J point.

Once at the C-J point, the explosive's energy is all released, ie. the burn fraction, $F$, is 1 . The reaction products now expand down the adiabat, a line of no entropy change. The problem is entirely a function of $P, v$ and energy and is size-independent. The JWL and the 1-D table are hydrocode EOS's that are based on C-J Theory.

\section{Energy Partition in C-J Theory}

The eneriges in C-J Theory are worth studying, just to see where they belong. We rewrite Eq. 2 a different way at the C-J point to see where the parts come from: 2,3

$$
\rho_{o} D(A t)\left[E_{m}+\frac{u_{p}^{2}}{2}-E_{o m}\right]=P u_{p}(\text { At })
$$

where $A$ is the cross sectional area, $t$ the time, $E_{m}$ the total internal (adiabat) energy in units per gram, and $E_{0 m}$ the chemical energy in units per gram. The right side is the PdV work done on the sample by compressing it. The left side is the resulting particle velocity and energy delivery in a section moving with the detonation velocity, $D$. We remove (At) and substitute for the particle velocity $u_{p}=D(1-v)$ from Eqs. 1 and 2 and $\rho_{0} E_{m}=E$ (energy/unit volume) to get the total internal energy:

$$
E=E_{0}+P(1-v)-\frac{\rho_{o} u_{p}^{2}}{2}
$$

This says that the total mechanical energy of compression is $P(1-v)$. The total energy put in from mechanical and chemical sources is

$$
\mathrm{E}(\text { total })=E_{0}+P(1-v)
$$

We now take Eqs. 1 and 2 and exclude $u_{p}$. We obtain the C-J relation 


$$
\frac{1}{2} \rho_{0} u_{p}^{2}=\frac{1}{2} P_{c j}\left(1-v_{c j}\right)
$$

This says that the kinetic energy of the explosive particle velocity is one-half the total Pdv energy, ie. our usual energy of compression, $\mathrm{E}_{\mathrm{c}}$. So half the Pdv energy goes to kinetic energy and half to internal.

The entire internal energy goes into maintaining the wave velocity. We write the C-J relation

$$
E_{s}(c j)=\frac{1}{2} \rho_{0} \lambda\left(1-v_{c j}\right)\left(D^{2}-u_{p}^{2}\right)
$$

This energy moves a "particle" of mass $\rho_{0} \lambda\left(1-v_{c j}\right)$, where we might expect the coefficient $\lambda$ to be near 1 , the depth of the compression wave being pushed through the material.

The total detonation energy becomes

$$
E_{o}=\frac{\rho_{0}}{2}\left[\lambda\left(1-v_{j}\right)\left(D^{2}-u_{p}^{2}\right)\right]-\frac{\rho_{o}}{2} u_{p}^{2}
$$

Using Eqs. 1 and 2, we exclude $u_{p}$ to get

$$
E_{0}=\frac{\rho_{0} D^{2}}{2}\left\{\lambda\left(1-v_{j}\right)\left[1-\left(1-v_{j}\right)^{2}\right]-\left(1-v_{j}\right)^{2}\right\}
$$

From Table 1-1, we find that $\left(1-v_{j}\right) \lambda$ is roughly inversely proportional to $\rho_{0}$, we get the important approximation

$$
E_{0} \sim D^{2}
$$

This may be extended to the same explosive with size effects, where $\mathrm{E}_{0}$ becomes a smaller average detonation energy, $E_{\mathrm{a}}$, which creates the smaller detonation velocity, $U_{\mathrm{s}}$. The Inverse Radius size effect theory depends on this relation.

We may also use the Rayleigh Line definition to convert Eq. 9 to 


$$
\left.E_{o}=\frac{P_{j}}{2}\left\{\lambda 1-\left(1-v_{j}\right)^{2}\right]-1\right\}
$$

which produces

$$
P_{j} \sim \rho_{0} E_{0}
$$

We previously noted that "efficient" explosives have a high value of $P_{c j} / E_{0} .{ }^{4}$ This category includes the best military explosives, which are not only explosive but have a high value of $E_{0}$ as well. A equally valid but more general measure of efficiency at the C-J point is

$$
f_{c j}=\frac{P_{c j} v_{c j}}{E_{s}(c j)}
$$

Table 1-1 lists the C-J properties for a wide assortment of explosives, along with their CHEETAH-calulated detonation point values. In Fig. 1-2, we show the cylinder jump-off velocities divided by $E_{0}$ as a function of the CHEETAH $f_{c j}$. Most of the cylinders were full-wall with some half-wall, but the jump-off velocity is largely independent of the metal plate thickness. Although most of the jump-off data is old streak camera data, we see that $f_{c j}$ measures the efficiency of jumpoff. A larger value of $f_{c j}$ also translates to a larger detonation volume, i.e.

$$
v_{c j} \approx 0.67+0.052 f_{c j}
$$

This seems surprising, but efficient explosives produce a energized product mixes that resist compression. In contrast, an inert substance can be squeezed to much smaller volumes because no chemical energy exists to re-expand the material.

In Eq. 8, the "mass" $\lambda(1-v)$ corresponding to the detonation wave is similiar to the effective mass of a hole in a semiconductor, which can be smaller or greater than the mass of an electron. The constant $\lambda$ is listed in Table 1-1 and shown in Fig. 1-3. We see that efficient explosives (those with large f) are "lighter" in their detonation masses while inefficient explosives have considerably "heavier" masses. 
In Table 1-1, we also note the percent of total energy at the detonation point that is kinetic, i.e. $E_{C}\left(2 E_{c}+E_{S}\right)$. These numbers tend to be higher for the high-f, efficient explosives. Also, the numbers appear to approach $25 \%$ as an upper limit.

\section{ZND Theory and the Existence of the C-J Point}

This is a World War II expansion of C-J Theory. It takes into account that most explosives do not react instantly but detonate over a period of time. The shock wave compresses the mixture of the initial explosive plus some of its products to a pressure spike that is higher than the C-J pressure. For real explosives, where a wall moves and the volume increases, we may consider two cases.

The first case is shown in Fig. 1-1b. Here, the solid, unreacted explosive is shocked and compressed and is driven up the unreacted Hugoniot to the spike point. Once there, it starts to react and it moves down the Rayleigh Line to the C-J point. At the C-J point, the explosive is all burned $(F=1)$ and the products are in thermodynamic equilibrium. This occurs only for explosives for which the shock wave thickness (about $1 / 4 \mathrm{~mm}$ - see Appendix B) equals the reaction zone thickness. This occurs only for PETN in normal experiments with imperfect confinement. In fact, no one has ever seen the spike for PETN- only the C-J point, which is why it was selected as the standard for the Cylinder Test (see Chapters 2 and 3). HMX and CL-20 are on the edge of this range with visible spikes but could be brought into it with heavier confinement.

Almost all explosives fall into the second ZND category shown in Fig. 1-1c. The explosive is compressed up to a spike point, which is shown by a square because a small amount of reaction has taken place even up to this point. For this explosive, the reaction zone is much longer than the skinny shock wave width. So the explosive continues to react long after the shock wave has passed and at pressures far below the spike. The C-J point does not exist at all. What we have is a release curve that is not really an adiabat because energy is being emitted. Because the spike pressure is greater than the C-J pressure, then in Eq. 2, $\rho_{0}$ and D are unchanged so that the particle velocity will be higher at the spike than at the C-J point. The particle velocity, $u_{p}$, decreases until we reach the sonic point where

$$
C_{o}+u_{p}=D
$$


where $\mathrm{C}_{0}$ is the speed of sound. The distance from the sonic point to the detonation front will be the effective reaction zone length. The energy from the this zone can be transmitted forward to set the properties of the detonation front.

Behind the sonic point, the explosive may burn but the result is not seen in the detonation velocity or curvature. Eventually, everything does burn, and the distance to this point may be called the true reaction zone. It is evident that the true reaction zone will be longer than the effective reaction zone, maybe much longer. Once $F=1$, the further release is a true adiabat.

No one agrees as to what an "ideal" explosive is. We might try this: an ideal explosive follows ZND theory with a true C-J point under the condition of practical heavy confinement. This places PETN, HMX and CL-20 in the ideal category. A non-ideal explosive never has a true C-J point even with practical heavy confinement. This includes all composite explosives and probably TATB.

Fig. 1-4 illustrates this issue. The jump-off velocities of LX-14 and LX-17 pushing copper and tantalum plates were measured using Fabry velocimetry. 5 These were converted to detonation pressures using the impedance equations (see Appendix B). They are plotted as a function of the thickness of the metal plate. $\mathrm{LX}-14$ is close to ideal., because the spike at 44 to $48 \mathrm{GPa}$ is seen through very thin foils. The calculated pressure then quickly drops to about $36 \mathrm{GPa}$, from which it decreases slowly. The drop occurs at about $0.25 \mathrm{~mm}$, which is taken as the approximate reaction zone length (even though we measured it in metal). The lower curve, extrapolated back to zero thickness, is taken as the "C-J" pressure of LX-14. This is how the first quantitative detonation pressure was historically obtained. The result agrees with the 35 to $38 \mathrm{GPa}$ C-J point calculated from the thermochemical codes.

LX-17 is a different story. The pressure declines slowly from $36 \mathrm{GPa}$ for thin foils to $34 \mathrm{GPa}$ for thick plates. The thermochemical code C-J point is 27 to $28 \mathrm{GPa}$, so we are way high. The fact that no slope change appears is what led to the conclusion that the reaction zone is greater than the maximum $2.5 \mathrm{~mm}$-thick plate. But there is still a problem. Suppose the reaction zone were $3 \mathrm{~mm}$ long with the spike at one end and the C-J point at the other. Then, we would expect $35 \mathrm{GPa}$ for thin plates and maybe $28 \mathrm{GPa}$ for the thickest. We see no such rapid decline. The answer comes if we assume that no C-J point exists at all, and that we decline from a single detonation pressure, which comes from the largely unburned spike. 
For a $Z$ ND explosive, the energy $E_{0}$ comes out over a distance, and we would not expect the energy partition equations for C-J Theory to work except for one. This one is reasonable at steady state:

$$
E_{0} \sim D^{2}
$$

This should work because the detonation velocity is constant across the detonation front and is created by all or part of the chemical reaction behind it. This relation will in Chapter 6 become the underpinning for the theory of the size effect and detonation front curvature.

\section{Definition of the JWL}

The most used Equation of State is the JWL, which is usually used to define the Principal Adiabat, i. e. the steady state expansion of the reaction product gases. The adiabat pressure is defined by

$$
P_{s}=A \exp \left(-R_{1} v\right)+B \exp \left(-R_{2} v\right)+\frac{C}{v^{1+\omega}}
$$

where $A, B, C, R_{1}, R_{2}$ and $\omega$ are constants. If it is an adiabat (with constant entropy), then it may be integrated to give the total internal energy

$$
E_{s}=-\int P_{s} d v=\left(\frac{A}{R_{1}}\right) \exp \left(-R_{1} v\right)+\left(\frac{B}{R_{2}}\right) \exp \left(-R_{2} v\right)+\frac{C}{\omega v^{\omega}}
$$

We may now eliminate $C$ between the two equations to get the JWL form used in production hydrocodes

$$
P_{s}=A\left(1-\frac{\omega}{R_{1} v}\right) \exp \left(-R_{1} v\right)+B\left(1-\frac{\omega}{R_{2} v}\right) \exp \left(-R_{2} v\right)+\frac{\omega E_{s}}{v}
$$

$\mathrm{E}_{\mathrm{S}}$ is always positive, starting with a large number at $\mathrm{v}_{\mathrm{j}}$ and declining to zero at some large volume. Also $\mathrm{E}_{\mathrm{S}}=\mathrm{E}_{\mathrm{O}}-\mathrm{E}_{\mathrm{d}}$. We use the physicists' convention, where $\mathrm{E}_{\mathrm{O}}$ is positive. Hence, the detonation energy, $E_{d}$, is negative for small volumes and positive for large volumes. At $v_{j}, E_{d}$ equals the energy of compression, $\mathbf{E}_{\mathrm{c}}$. Eq. 19 requires the energy to be inserted at every time step, which makes codewriters feel better about its conservation. However, the energy is computed by adding or subtracting 
increments on each cycle, so that the quality of conservation depends ultimately on the smallness of the steps.

Eq. 19 is used only to set the pressure. The detonation velocity, D, is used to set the timing for the program burn part of the burn package. For the beta burn part, the code needs to be told the detonation point so it can calculate compression. One might give it the detonation volume $\mathrm{v}_{\mathrm{j}}$ directly, which is what it really wants or $P_{j}$ as is done in DYNA2D. Another common entry in LLNL production codes is $\Gamma_{j}+1$ sometimes called $\beta$ or bhe, which is defined by,

$$
\Gamma_{j}+1=\frac{v_{j}}{P_{j}}\left(\frac{\partial P_{j}}{\partial v_{j}}\right)_{S}+1=\frac{1}{1-v_{j}}
$$

$\Gamma_{\mathrm{j}}$ is the adiabatic gamma at the detonation point, and it varies only between 3.5 to about 4.2. The " $\mathrm{j"}$ means that the detonation point is probably not a true C-J point but is an approximation depending on how it was generated.

The hydrocode doesn't care if the actual detonation point is reached or not. It will be reached only in the case of heavy containment and with zoning to convergence. The energy dumped into the explosive is $\mathrm{FE}_{0}$, which rises to $\mathrm{E}_{0}$ after the shock wave has passed through. The reason why nobody cares about the detonation point is that $E_{0}$ is more or less the same for a large section of the upper Rayleigh Line. For LX-14 and the $381 \mathrm{JWL}$, we find these results from the detonation point on down:

\begin{tabular}{|c|c|c|}
\hline $\begin{array}{l}\text { Det } \\
\text { Volume, } \\
\text { v }\end{array}$ & $\begin{array}{l}\text { Det } \\
\text { Pressure, } \\
\mathrm{P}_{\mathrm{j}}(\mathrm{GPa})\end{array}$ & $\begin{array}{l}\text { Det } \\
\text { Energy, } \\
\mathrm{E}_{\mathrm{o}}(\mathrm{kJ} / \mathrm{cc})\end{array}$ \\
\hline 0.734 & 38.1 & 10.1 \\
\hline 0.75 & 35.8 & 10.1 \\
\hline 0.78 & 32.1 & 10.0 \\
\hline 0.80 & 29.8 & 10.0 \\
\hline 0.82 & 27.7 . & 9.9 \\
\hline 0.84 & 25.8 & 9.8 \\
\hline
\end{tabular}

We see that $\mathrm{E}_{\mathrm{O}}$ remains almost constant despite a huge drop in the detonation pressure. This is because compressing less means that the system restores itself more easily while putting out almost the same chemical energy. 


\section{Adiabatic and Gruneison Gamma}

In the reacted explosive, there are two important derivitives. Adiabatic gamma was just defined at one point. More generally, it describes the derivitive along the adiabat and is

$$
\Gamma=\frac{\mathrm{v}}{\mathrm{P}_{\mathrm{s}}}\left(\frac{\partial \mathrm{P}_{\mathrm{s}}}{\partial \mathrm{v}}\right)_{\mathrm{s}}
$$

The second derivitive is the Gruneison gamma. This is defined by

$$
\Gamma_{\mathrm{G}}=v\left(\frac{\partial \mathrm{P}_{\mathrm{s}}}{\partial \mathrm{E}_{\mathrm{s}}}\right)_{\mathrm{v}}
$$

The partial derivitive of pressure with internal energy is at constant volume, so that this is a derivitive that is transverse with respect to the adiabat.

It is believed that both $\Gamma$ and $\Gamma_{G}$ increase slowly as the pressure of the reacted explosive increases. Fig. 1-5 shows the results from the JWL. At the detonation point, we see that $\Gamma$ is moving in the opposite direction from what we expected. This is the result of the highest pressure exponential, which has the same structure as its derivitive. For the $A$ term, $\Gamma \approx v$ and $\Gamma$ is increasing with the volume. The next exponential term starts $\Gamma$ down again. Hence, $\Gamma$ for a JWL forms a set of two peaks, all the result of the JWL function. At large volume we get ${ }^{6}$

$$
\Gamma=\omega
$$

To sum it up for the JWL, $\Gamma$ increases with increasing pressure for awhile; then it decreases. $\Gamma$ remains constant at the low pressure value throughout. Now, it is probably true that this JWL behavior is unphysical on both counts. But it is not yet clear that this greatly hampers its performance as an EOS, because many problems spend little time at high pressure. An on-going question for those who like one-track EOS's is how important the behavior of the two gammas really is in the construction of a one-track EOS.

\section{The One-Dimensional Table}


If it is desired to make the gammas behave more physically, then the one-dimensional LookUp Table is an alternate EOS that has been placed into LLNL codes. ${ }^{7}$ We write the equation

$$
P_{s} v=f(v) E_{s}
$$

where $P_{S}$ and $E_{S}$ are the pressure and internal energy of the adiabat. This is the same $f$ that appeared above as a measure of detonation efficiency. The differential form is

$$
\frac{d P_{s}}{d v}=\frac{E_{s}}{v}\left(\frac{\partial f}{\partial v}\right)+\frac{f}{v}\left(\frac{\partial E_{s}}{\partial v}\right)-\frac{f E_{s}}{v^{2}}
$$

We substitute Eq. 25 and $\partial \mathrm{E}_{\mathrm{S}} / \partial \mathrm{v}=-\mathrm{P}_{\mathrm{S}}$ to obtain

$$
\frac{d \ln P_{s}}{d v}=\frac{1}{f}\left(\frac{\partial f}{\partial v}\right)-\frac{1}{v}(f+1)
$$

This can be converted into $\Gamma$ to give

$$
\Gamma=-\frac{v}{f}\left(\frac{\partial f}{\partial v}\right)+(f+1)
$$

Also, the Gruneison gamma is

$$
\Gamma_{G}=f+E_{\mathrm{g}}\left(\frac{\partial f}{\partial E_{s}}\right)_{v}
$$

If $f$ is a function of $v$ only, which is the simplest assumption in the absence of transverse knowledge, then $\Gamma_{G}=f$.

Eq. 27 may be numerically integrated to provide

$$
\ln P_{s}=\int_{v(1)}^{v}\left\{\frac{1}{f}\left(\frac{\partial f}{\partial v}\right)-\frac{1}{v}(f+1)\right\} d v
$$

where the integration starts at the large 1 atm volume, $v(1)$, which is several thousand, and proceeds to some small volume, $\mathrm{v}$, which can be the detonation volume or beyond. Thus, all pressures and 
energies are derived on a spreadsheet. The actual input to the code, however, is a list of $\eta$ (inverse of the volume, $v$ ) and $f$ and the code does the rest. The need for a numerical integration makes the Look-Up Table more abstract than the JWL.

We return to adiabatic $\Gamma$ in Fig. 1-5. Also shown in the figure is the direct result from the thermochemical code CHEQ. A dip appears as the result of a calculated phase transition in the products. The result is to produce a nearly constant value over a large expanse of volume. If this is considered to be a realistic description of the explosive, then only a table format will work.

The current procedure for creating a custom look-up table is to take an adiabat from CHEQ and directly generate a table of $f$ values. A hydrocode run is made on a compressive geometry. If the energy is off by $10 \%$, then the makers of CHEQ are asked to recalibrate their code for this material and the process is repeated. The problems will always be at high pressures and small volumes. If the answers are within 5\%, then the table is massaged. Adjustments are made from the C-J point on down to low pressures. A typical change is to increase the the C-J pressure and the pressure just below C-J, because CHEQ usually has too little energy in this region. This is done by either deepening the phase transition just below C-J or moving the phase transition to a lower volume expansion. This process will modify the adiabat everywhere, including at lower pressures.

A disadvantage of this process is the need for the subjective judgement by the maker of the Table. At present, there is no way to make this process as straight-forward as the derivation of a cylinder JWL.

\section{Program Burn/ Beta Burn Package}

The LLNL production codes have an overlaid program burn/ beta burn turn-on package. The program burn turns on $F$ in a given zone at time $t_{0}$, based on the distance to the zone and the detonation velocity, $\mathrm{D}$. The burn fraction in each zone, $\mathrm{F}$, is calculated by

$$
F=\frac{t-t_{0}}{\Delta t}
$$

where $t$ is time and $\Delta t$ is the approximate time for the detonation wave to cross the diagonal of a zone. It is possible for Eq. 31 to turn on linearly if it is increased very slowly, but often F steps from 0 to 1 in one zone. 
The beta burn calculates the burn fraction from

$$
F=\frac{1-v}{1-v_{j}}=\left(\Gamma_{j}+1\right)(1-v)
$$

The name beta burn comes from calling $\Gamma_{j}+1$ beta. On each cycle and in each zone, the two F's from Eqs. 31 and 32 are compared and the bigger one is used. Thus the program and beta burn mechanisms are overlaid.

The code also contains an artifical pressure, Q (usually called the "artificial viscosity"). This gets turned on during a shock when the pressure is changing rapidly. It goes away when the pressure change is slow. It provides extra pressure to accelerate mass, which increases the rate at which the code problem turns on. This is shown in Fig. 1-6 for a point $30 \mathrm{~mm}$ into a piece of LX-14. A point detonation is used so that the pressure rises slowly. The boost given by $Q$ as the zones compress turns the total code pressure, $P+Q$, on sooner. We have seen in a section above that the shock wave rolls through quickly but the chemical reaction can go on for a long time. We have a fast physical process overlaid on a generally slower chemical one. The hydrocode does it just this way: The artifical viscosity ensures the existence of the shock wave but nothing else. If the system needs to go "off-adiabat", ie. onto a track not described by the EOS, the artifical viscosity has no ability to add the necessary entropy.

The time for a burn package to turn on is a function of the zoning. The turn-on of detonation velocity is the best indicator in a real explosive but is hard to get from a code. Thus, we use the total pressure, $P+Q$, instead. The fact that $Q$ pushes like a real explosive means that $P+Q$ should cause the movement of the detonation front. For a point detonation (with no flyer), we may write

$$
(P+Q) \approx(P+Q)_{0}+\Delta(P+Q)\left[1-\exp \left(-t / \tau_{\mathrm{SS}}\right)\right]
$$

Here, $\Delta(P+Q)$ is the difference between the minimum value, $(P+Q)_{0}$, and the steady state value of $0.373 \mathrm{Mb}$ for $\mathrm{LX}-14$. The $1 / \mathrm{e}$-pressure will be about $0.300 \mathrm{Mb}$ for the point detonation. Near steady state, $Q$ will approach zero and $P+Q$ will be all $P$. For planar LX-14 in the 1-D code with monatonic $Q$, the time constants to $P+Q$ steady state are: 


\begin{tabular}{cl} 
zones $/ \mathrm{mm}$ & $\tau_{\mathrm{sS}}(\mu \mathrm{s})$ \\
\hline 1 & 1.2 \\
2 & 0.55 \\
5 & 0.20 \\
10 & 0.10 \\
20 & 0.053 \\
40 & 0.028 \\
80 & 0.016
\end{tabular}

The total times to steady state will be 4 or 5 times these values. This could be $5 \mu$ s at 1 zone/mm. At $8.8 \mathrm{~mm} / \mu \mathrm{s}$ for LX-14, this amounts to a long $44 \mathrm{~mm}$ distance. LX-14 is itself much faster than this, so one has to be sure that the proper speed is coming from the zoning.

The jump-off velocity is a high frequency edge, so we expect zoning to be critical here as well, as shown in Fig. 1-7. Samples phm85007 and phm85008 were $39 \mathrm{~mm}$ thick LX-14 with $0.762 \mathrm{~mm}$ ( $30 \mathrm{mil}$ ) tantalum driven by a $10 \mathrm{mil}$ Mylar flyer at $4.3 \mathrm{~mm} / \mu \mathrm{s}$. The experiment is modeled with 1,5 and $20 \mathrm{zone} / \mathrm{mm}$ in the high explosive with the metal zoning being left constant. We see that 1 zone/mm is far too coarse, 5 is all right but 10 or more is needed to get it right. With more emphasis on the EOS near the detonation point, finer zoning appears necessary.

The codes slso allow initiation using a flyer or a pressure pulse, as shown for planar LX-14 in the 1-D code in Fig. 1-8. Shown is the underdriven point detonation case plus two overdriven cases based on the $0.25 \mathrm{~mm}$ (10 mil) Mylar flyer: a flyer moving at $10 \mathrm{~mm} / \mu \mathrm{s}$ and a pressure pulse $0.02 \mu \mathrm{s}$ long at $0.80 \mathrm{Mb}$. Both drive the explosive to near $1 \mathrm{Mb}$; then the pressure decays back to the expected $0.373 \mathrm{Mb}$ detonation point for the $\mathrm{A}=7.65 \mathrm{JWL}$. The detonation velocities for the overdriven case also decline to near the steady state value. The point detonation and the flyer case both lock onto the program burn detonation velocity. The pressure pulse approaches a slightly low but probably acceptable velocity derived from its own beta-burn calculation.

\section{Terminilogy of Detonation Points}

The term "C-J point" is used very loosely in the explosives community to describe the start of a detonation. We shall try to distinquish between the various sources and meanings of these points. For "detonation pressure," as an example, we shall use these various subscripts. 
$P_{c j}$, the C-J pressure, is the equilibrium pressure as calculated in a thermochemical code (eg. CHEQ or CHEETAH). It may exist in the few explosives whose reaction zones are as wide as the shock wave.

$P_{j}$ is an EOS detonation pressure, obtained either from a cylinder test or by fitting a hydrocode to a plate shot.

$\mathrm{P}_{\mathrm{e}}$ is the pressure obtained by the use of impedance equations without further analysis. When the plate thickness is thin, the pressure seen is $\mathrm{P}_{\mathbf{k}}$, the spike pressure, for a near-ideal explosive. When the plate thickness is equal to the reaction zone for a near-ideal explosive, the pressure is thought to be close to $P_{c j}$ for an ideal explosive.

$\mathrm{P}_{\mathrm{k}}$. This is the first high, von Neumann spike pressure seen in the thin plate test or as found in the I\&G code. It results from the initial compression of the unburned or partly burned explosive. As the reaction continues, this pressure drops down the Rayleigh Line toward the detonation point.

$P_{S}$ is the pressure on the Principal Adiabat at any point on it. The detonation point is the highest pressure point on the curve.

$\mathrm{P}_{\mathrm{h}}$ is the pressure on the explosive Hugoniot. The detonation point is the lowest pressure point on this curve.

\section{Units, etc.}

Mbar and GPa units are mixed thoughout this report. In the first, pressures are in $\mathbf{M b}$; energies in $\mathrm{Mb} \cdot \mathrm{cc} / \mathrm{cc}$ and velocity in $\mathrm{cm} / \mu \mathrm{s}$. In the second set of units, we have GPa, $\mathrm{kJ} / \mathrm{cc}$ and $\mathrm{mm} / \mu \mathrm{s}$.

Conversions may be made as follows:

$$
\begin{array}{lll}
\text { Pressure: } & \text { Mbar } \rightarrow->\text { GPa } & \text { multiply by } 100 \\
\text { Energy: } & \text { Mbar·cc/cc } \rightarrow \text { kJ/cc } & \text { multiply by } 100 .
\end{array}
$$

A calorie becomes a joule by multiplying by 4.184. TIGER or the long output file of CHEETAH give atmospheres for pressure. A Matm goes to a GPa by multiplying by 101.325 . 
Pressure in the Rankine-Hugoniot momentum relation is luckily maintained with the following mongrel equality:

$$
\mathrm{GPa}=(\mathrm{g} / \mathrm{cc})(\mathrm{mm} / \mu \mathrm{s})^{2}
$$

Pressure and energy may be compared using

$$
\mathrm{GPa}=\mathrm{kJ} / \mathrm{cc}
$$

\section{References}

1. P. C. Souers and L. C. Haselman, Jr., Detonation Equation of State at LLNL, 1993, LLNL Report UCRL-ID-116113 (March, 1994).

2. Ia. B. Zeldovich and A. S. Kompaneets, Theory of Detonation (Academic Press, New York, 1960), pp. 10-11.

3. Ya. B. Zel'dovich and Yu. P. Raizer, Physics of Shock Waves and High-Temperature

Hydrodynamic Phenomena, W. D. Hayes and R. F. Probstein, ed. (Academic Press, New York, 1967), p. 705 .

4. Reference 1, p. 5-3.

5. Reference 1 , pp. 6-15, 6-18 to 6-21.

6. Pier Tang, Los Alamos National Laboratory, private communication, 1995.

7. The function used in the Look-Up Table is similiar to the EOS described by W. C. Davis of LANL in "Equation of State for Detonation Products," The Tenth International Detonation Symposium. Boston. MA, July 12-16, 1993, pp. 112 -113 of Paper Summaries; private communication, 1993. The LLNL and LANL use of this function occured independently. A discussion of both $\gamma$ and $\Gamma$ is included and an off-adiabat form is given. 


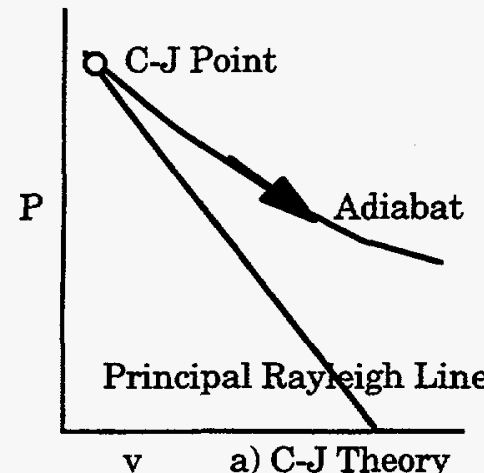

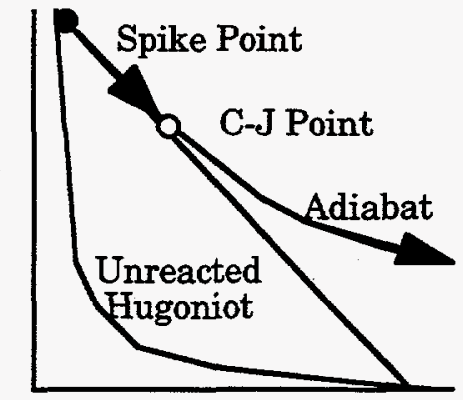

b) Unusual ZND Theory

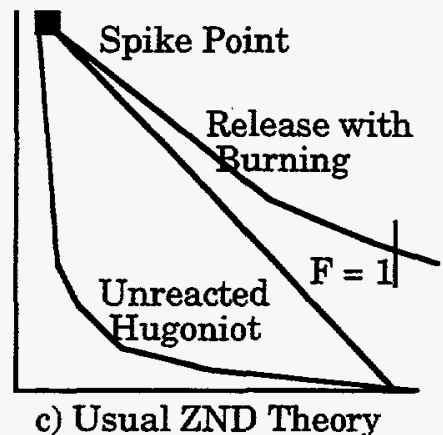

c) Usual ZND Theory

Fig. 1-1. Pressure (Y-axis) - relative volume (horizontal axis) plots of the major explosive theories.

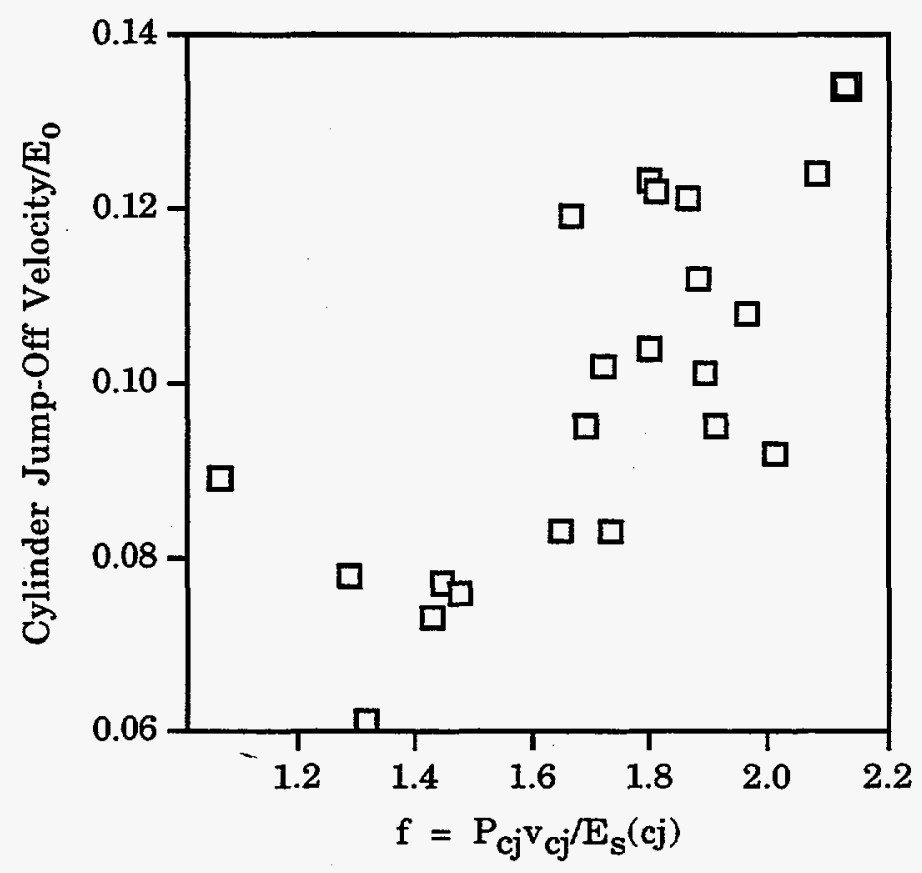

Fig. 1-2. Cylinder Test jump-off efficiency plotted as a function of $f$, showing a definite linear trend. 


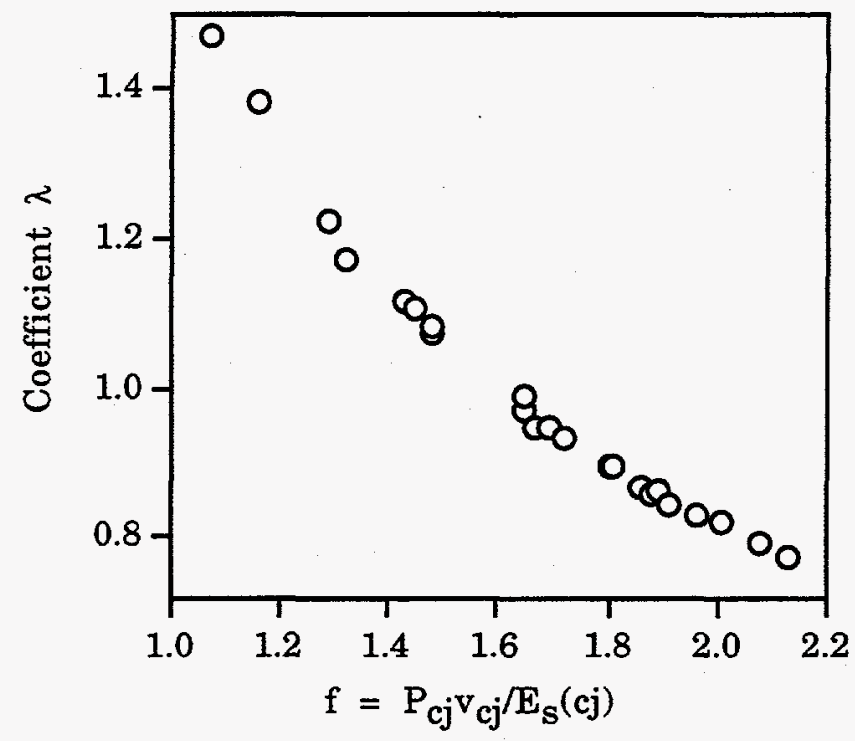

Fig. 1-3. Coefficient $\lambda$, which describes the effective mass of the detonation wave, is shown to be a function of $f$.

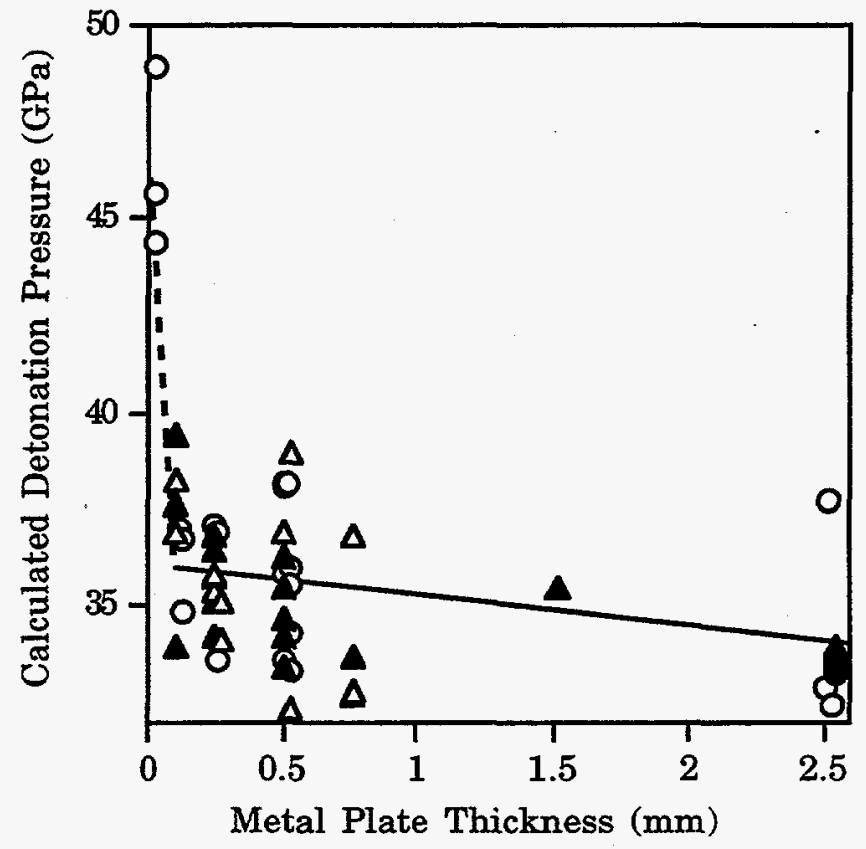

Fig. 1-4. Detonation pressures as calculated from impedance matching plotted as a function of metal plate thickness. Electric guns were used to initiate; Fabry velocitmetry was used to measure the jump-off velocities. The samples are: LX-14 with $\mathrm{Ta}(\Delta)$ and $\mathrm{Cu}(\mathrm{O}) ; \mathrm{LX}-17$ with $\mathrm{Ta}(\Delta)$ and $\mathrm{Cu}$ (O). Both explosives have the same 36 to $34 \mathrm{GPa}$ curve. 


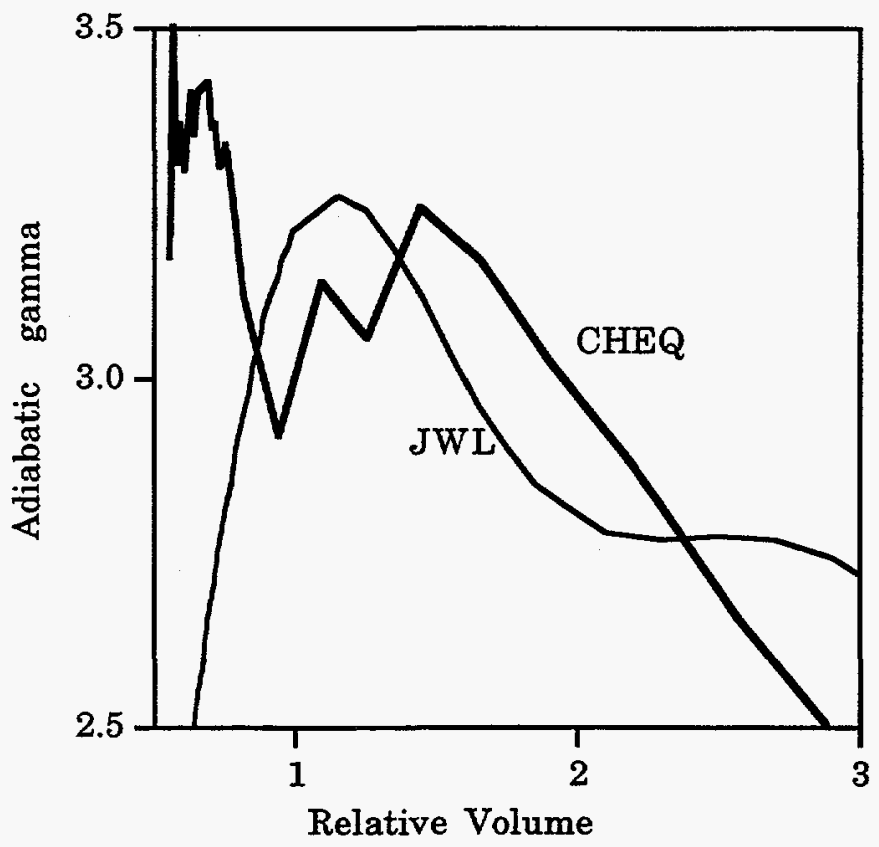

Fig. 1-5. Adiabatic $\Gamma$ for $L X-14$ from the $A=7.65 \mathrm{JWL}$ and the thermochemical code CHEQ. The $J W L ' s$ declining value at small volumes is the non-physical issue to be considered.

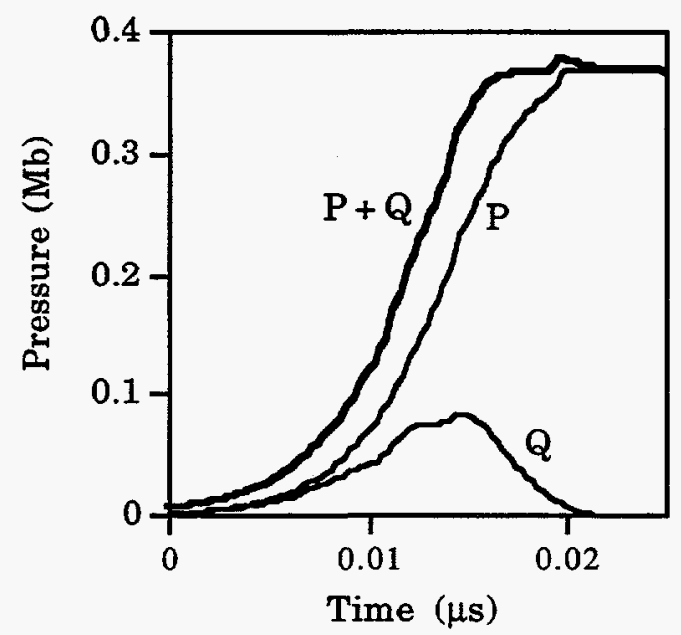

Fig. 1-6. Effect of the artifical pressure $Q$ in a given cell. $Q$ turns on when the zone compresses and advances the timing. 


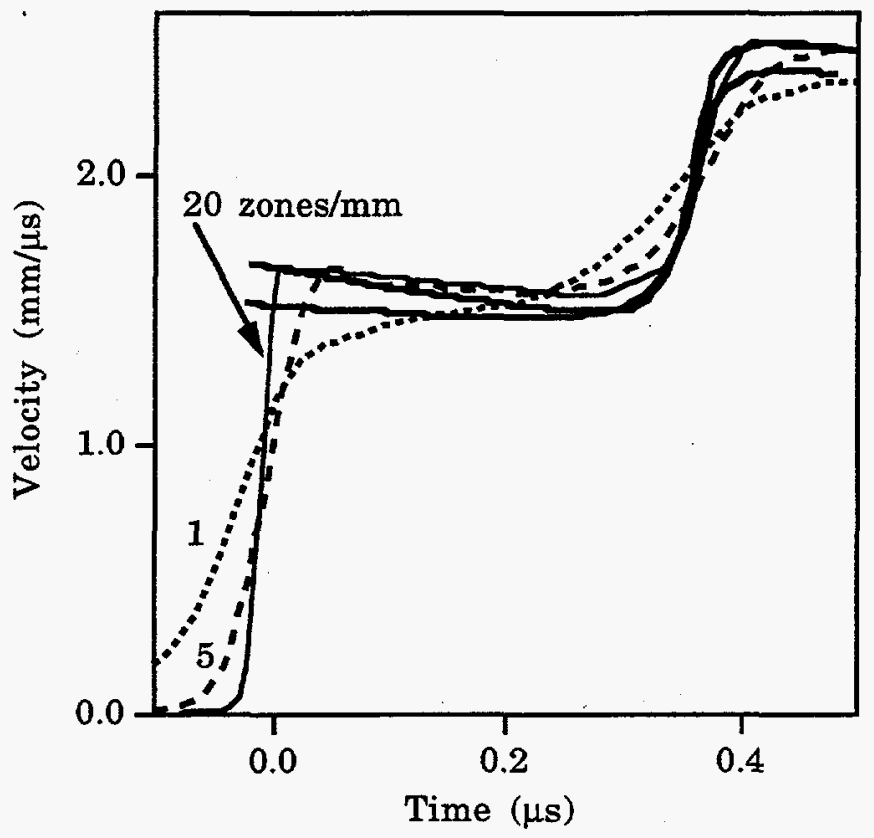

Fig. 1-7. Jump-off velocities as a function of zoning for $39 \mathrm{~mm} \mathrm{LX}-14$ with a $0.762 \mathrm{~mm}$ (30 mil) tantalum plate. The data are the two heavy curves. At least 5 zones $/ \mathrm{mm}$ are needed to get close; 10 to $20 \mathrm{zones} / \mathrm{mm}$ is better.

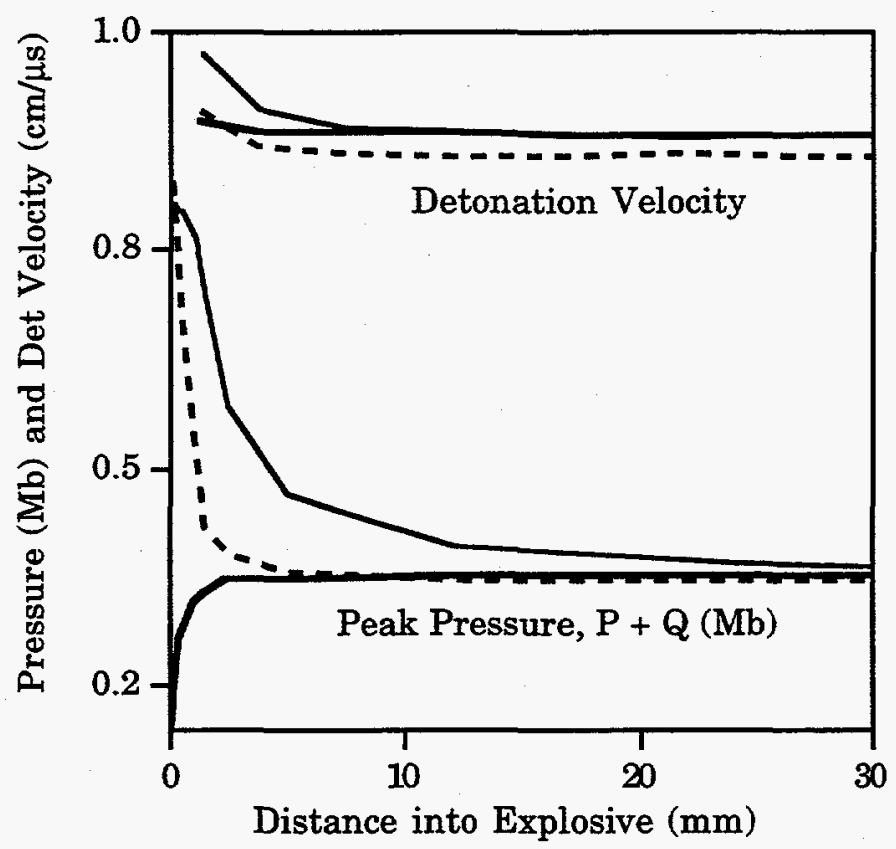

Fig. 1-8. Motion of the 1-D code toward steady state for planar LX-14: underdriven point detonation (heavy line); overdriven with a Mylar flyer at $10 \mathrm{~mm} / \mu \mathrm{s}$ (light full line) and overdriven with a pressure pulse $0.02 \mu \mathrm{s}$ and $0.80 \mathrm{Mb}$ (dashed). The peak pressure is shown which is virtually all $P$. The detonation velocity for the pressure pulse is slightly below the program burn value. 
Table 1-1. Results of the energy partition studies of explosives at the C-J detonation point, as described by CHEETAH. Cylinder jump-off velocity data is also included. the explosives are ordered according to descending $f=P_{c j} v_{c j} / E_{s}(c j)$.

\begin{tabular}{|c|c|c|c|c|c|c|c|c|c|}
\hline & $\begin{array}{c}\text { Den- } \\
\text { sity } \\
\rho_{0} \\
(\mathrm{~g} / \mathrm{cc})\end{array}$ & $\begin{array}{l}\text { Pres- } \\
\text { sure } \\
P_{c j} \\
(\mathrm{GPa})\end{array}$ & $\begin{array}{c}\text { Det } \\
\text { Vel, D } \\
(\mathrm{mm} / \\
\mu \mathrm{s})\end{array}$ & $\begin{array}{c}\text { Det } \\
\text { Energy } \\
\mathrm{E}_{\mathrm{o}} \\
(\mathrm{kJ} / \mathrm{cc})\end{array}$ & $\begin{array}{c}\text { C-J } \\
\text { Volume } \\
\text { v }_{\text {cj }}\end{array}$ & $f$ & $\lambda$ & $\begin{array}{c}\text { Cyl } \\
\text { Jump- } \\
\text { Off } \\
\text { Velocity } \\
(\mathrm{mm} / \mu \mathrm{s})\end{array}$ & $\begin{array}{c}\% \\
\text { Kinetic } \\
\text { to Total } \\
\text { Energy }\end{array}$ \\
\hline AFX-902 & 1.74 & 24.8 & 8.08 & 6.40 & 0.782 & 2.13 & 0.77 & 0.86 & 22.9 \\
\hline TNM & 1.65 & 14.3 & 6.36 & 3.87 & 0.786 & 2.08 & 0.79 & 0.48 & 22.0 \\
\hline RX-23-AA & 1.42 & 23.5 & 8.73 & 6.61 & 0.784 & 2.01 & 0.82 & 0.61 & 21.7 \\
\hline $\mathrm{RX}-39-\mathrm{AB}$ & 1.94 & 41.0 & 9.38 & 11.37 & 0.760 & 1.91 & 0.84 & 1.08 & 23.2 \\
\hline $\mathrm{RX}-45-\mathrm{AA}$ & 1.75 & 24.0 & 7.78 & 6.76 & 0.774 & 1.96 & 0.83 & 0.73 & 22.3 \\
\hline HMX & 1.89 & 38.8 & 9.26 & 11.04 & 0.761 & 1.88 & 0.86 & 1.24 & 22.8 \\
\hline LX-17 & 1.91 & 26.8 & 7.72 & 7.83 & 0.763 & 1.86 & 0.86 & 0.95 & 22.4 \\
\hline $\mathrm{RX}-41-\mathrm{AB}$ & 1.86 & 34.1 & 8.90 & 9.93 & 0.768 & 1.89 & 0.86 & 1.00 & 22.2 \\
\hline $\mathrm{RX}-26-\mathrm{AF}$ & 1.84 & 30.6 & 8.26 & 9.12 & 0.757 & 1.80 & 0.89 & 1.12 & 22.4 \\
\hline LX-14 & 1.83 & 34.2 & 8.78 & 10.24 & 0.757 & 1.80 & 0.89 & 1.07 & 22.4 \\
\hline TATB & 1.83 & 26.2 & 7.75 & 7.92 & 0.761 & 1.81 & 0.89 & 0.97 & 22.1 \\
\hline $\mathrm{RX}-36-\mathrm{AH}$ & 1.83 & 33.3 & 8.52 & 10.30 & 0.750 & 1.73 & 0.93 & 0.85 & 22.3 \\
\hline RX-47-AA & 1.82 & 25.9 & 7.66 & 8.25 & 0.758 & 1.72 & 0.93 & 0.84 & 21.6 \\
\hline BTF & 1.85 & 36.5 & 8.58 & 11.12 & 0.732 & 1.67 & 0.95 & 1.32 & 23.4 \\
\hline RX-48-AA & 1.85 & 27.8 & 7.77 & 8.91 & 0.751 & 1.69 & 0.95 & 0.85 & 21.9 \\
\hline PETN & 1.76 & 30.8 & 8.51 & 10.39 & 0.758 & 1.65 & 0.97 & 0.86 & 20.9 \\
\hline HNB & 1.97 & 37.5 & 9.23 & 13.43 & 0.776 & 1.65 & 0.99 & & 19.3 \\
\hline HNS & 1.66 & 20.6 & 6.99 & 7.73 & 0.745 & 1.48 & 1.07 & 0.59 & 20.2 \\
\hline FEFO & 1.61 & 21.5 & 7.35 & 8.28 & 0.752 & 1.48 & 1.08 & 0.63 & 19.5 \\
\hline TNT & 1.63 & 18.8 & 6.80 & 7.38 & 0.751 & 1.45 & 1.11 & 0.57 & 19.4 \\
\hline PETN & 1.50 & 22.3 & 7.65 & 8.79 & 0.746 & 1.43 & 1.12 & 0.64 & 19.6 \\
\hline $\mathrm{NM}$ & 1.13 & 14.6 & 6.68 & 5.73 & 0.710 & 1.32 & 1.17 & 0.35 & 21.3 \\
\hline HMX & 1.19 & 14.4 & 6.76 & 6.28 & 0.735 & 1.29 & 1.22 & 0.49 & 18.9 \\
\hline PETN & 1.26 & 14.0 & 6.64 & 7.29 & 0.748 & 1.16 & 1.38 & & 16.3 \\
\hline HNS & 1.00 & 7.3 & 5.15 & 3.94 & 0.726 & 1.07 & 1.47 & 0.35 & 16.8 \\
\hline
\end{tabular}




\section{Chapter 2. JWL Construction}

\section{Cylinder JWL's: the Old Method}

The big problem with Principal Adiabat JWL's is that the numbers from one explosive can't be compared with those from another. The numbers are all over the map. There is no possibility of prediction and it probably ensures that the rate constants in Ignition \& Growth will also be all over the map. We shall attempt to create a global JWL description.

We first summarize the old cylinder JWL process as described in the previous report. It is based on the old $1.763 \mathrm{~g} / \mathrm{cc}$ PETN "standard" JWL:

$\begin{array}{ll}\mathrm{A} & 1032.158 \mathrm{GPa}=10.32158 \mathrm{Mbars} \\ \mathrm{B} & 90.57014 \mathrm{GPa}=0.9057014 \mathrm{Mbars} \\ \mathrm{C} & 3.72735 \mathrm{GPa}=0.0372735 \mathrm{Mbars} \\ \mathrm{R}_{1} & 6.0 \\ \mathrm{R}_{2} & 2.6 \\ \omega & 0.57 \\ \mathrm{P}_{\mathrm{j}} & 31.5 \mathrm{GPa}=0.315 \mathrm{Mbars} \\ \mathrm{D} & 8.274 \mathrm{~mm} / \mu \mathrm{s}=0.8274 \mathrm{~cm} / \mathrm{s} \\ \mathrm{E}_{0} & 10.8 \mathrm{~kJ} / \mathrm{cc}=0.108 \mathrm{Mbar} \cdot \mathrm{cc} \\ \Gamma_{\mathrm{j}}+1 & 3.832\end{array}$

The detonation pressure is very close to the $31 \pm 2 \mathrm{GPa}$ value considered to be the best such measurement. The total energy of detonation is also close to the $10.3 \mathrm{~kJ} / \mathrm{cc}$ value from the calorimeter. The data for the PETN standard are as follows:

\begin{tabular}{|c|c|c|c|c|}
\hline \multirow[b]{2}{*}{$\begin{array}{c}\mathrm{R}-\mathrm{R}_{0} \\
(\mathrm{~mm})\end{array}$} & \multirow[b]{2}{*}{$\mathbf{v}$} & \multirow{2}{*}{$\begin{array}{c}\mathrm{E}_{\mathrm{d}} \\
(\mathrm{PETN}) \\
(\mathrm{kJ} / \mathrm{cc})\end{array}$} & \multicolumn{2}{|c|}{ Velocity (mm/us) } \\
\hline & & & $\begin{array}{c}\text { PETN } \\
\text { half-wall }\end{array}$ & $\begin{array}{c}\text { PETN } \\
\text { full-wall }\end{array}$ \\
\hline 6 & 2.2 & 6.51 & 2.09 & 1.58 \\
\hline 12.5 & 4.1 & 7.87 & 2.28 & 1.72 \\
\hline 19 & 6.5 & 8.55 & 2.38 & 1.78 \\
\hline
\end{tabular}

From this, we can create a table that relates all measured velocity-squares to those of PETN. This makes the assumption that velocity-squared is always proportional to the energy of detonation. 
The old "3-point" method of JWL calculation seems so straight-forward that the psychology of the estimating process is ususally ignored. We start with the \%-difference of the unknown explosive with PETN at each of the three volumes from the velocity-squared. This data is fit closely- in fact, to better than it deserves, given that the scatter can be considerable. All other constants are made to fit this data by the following process.

1) We estimate $E_{0}$ and $P_{j}$. Our brains are calibrated for LX-17 and LX-14 at 26 and $38 \mathrm{GPa}$ and 7 and $10 \mathrm{~kJ} / \mathrm{cc}$. We estimate where our unknown explosive is linearly using these points. We could be somewhat more quantitative by using a CHEETAH $\mathrm{E}_{0}$, which will probably be within $5 \%$ (unless it is a composite). We could also get $P_{j}$ fairly close from

$$
P_{j}=\rho_{0} D^{2}\left(1-v_{j}\right) \approx \rho_{0} D^{2} / 4
$$

We know the density and detonation velocity and we set the detonation volume to be roughly 0.75 .

2) We set

$$
R_{1} \approx 4.5, R_{2} \approx 1.5, \omega \approx 0.3
$$

3) We now fiddle with $R_{1}, R_{2}, \omega, E_{0}$ and $P_{j}$ (but not $A, B$ and $C$, which are calculated) and look at the \%-agreement at each volume of the calculated JWL detonation energy, $\mathrm{E}_{\mathrm{d}}$, with that calculated using velocities from PETN. The three \%-agreement numbers are averaged and are zeroed out by our selection of constants, so we are really looking at the two extreme \%'s, one negative and one positive. In this fiddling, is it expected that the resultant $\mathrm{A}$ will bear a roughly linear relation with the explosive strength, eg. about $4 \mathrm{Mb}$ for ANFO and 8 to $12 \mathrm{Mb}$ for PETN. We also require that $A$ be 10 to 50 times $B$ and $B 10$ to 50 times $C$. Also, $4<R_{1}<7 ; 0.8<R_{2}<2$; and $0.25<$ $\omega<0.6$.

4) When the two extemes fall within $\pm 1 \%$, we are satisfied. Sometimes, by luck, they quickly fall to $\pm 1 / 2 \%$ or even better. Occasionally, we can't quite make it to $1 \%$. These are all better than the $\pm 5 \%$ actual error present in streak camera velocities and this is the cause of the many widelyvarying JWL's.

5) There is no reason why the above parameters have to be as described in the 3-point method. Sometimes, agreement with the data is not obtained and the poor quality of the streak- 
derived velocities is never blamed. So another way out is to let $R_{1}$ and $R_{2}$ together drift upward until agreement is reached. This happened with the PETN standard listed above.

Thus, the operator makes assumptions as to what the constants should be and stops when $1 \%$-agreement is reached for the first time.

\section{Observations from Data}

We now sum up the derived JWL's for 53 full-wall homogeneous and heterogeneous (but not composite) explosives. The averages and standard deviations are as follows. For $\mathrm{A}$ we have:

$$
\mathrm{A} / \mathrm{\rho}_{\mathrm{o}} \mathrm{D}^{2}=6.2 \pm 2.0
$$

and

\begin{tabular}{llllll} 
& $\mathrm{B}$ & $\mathrm{C}$ & & & \\
& $(\mathrm{GPa})$ & $(\mathrm{GPa})$ & $\mathrm{R}_{1}$ & $\mathrm{R}_{2}$ & $\omega$ \\
\cline { 2 - 6 } average & 11 & 1.6 & 4.6 & 1.2 & 0.42 \\
stdev & 17 & 0.9 & 0.5 & 0.4 & 0.11
\end{tabular}

These results confirm the structure we desire to impose. We recall that Urtiew and Hayes tried to relate all JWL's through the assumption that $A, B$ and $C$ are proportional to $\rho_{0} D^{2}{ }^{1}$ It appears that this is probably true only for $A$. A is really proportional to the detonation pressure, with $\rho_{0} D^{2}$ being closely related through the Rayleigh Line.

We note that our standard PETN is way out of line from these results. PETN has a funnylooking JWL, which we would have never obtained from our JWL-making process.

Next, most cylinder JWL's are based on old streak camera data. The process of differentiation introduces considerable error, so that the typical wall velocity fit to the JWL is

$$
\mathrm{u}(\mathrm{wall}) \sim 2 \pm 0.1 \mathrm{~mm} / \mu \mathrm{s} \text { or } \pm 5 \%
$$

So, an error of $\pm 5 \%$ is suspected. The practice of leading ourselves to the first $\pm 1 \%$ JWL fit probably assures that JWL's will have no relation to one another. 


\section{The Freeze Assumption}

We are going to get some help from thermochemical codes to set part of the JWL. First, we need to settle one issue: that of freezing the reaction products at some temperature. Traditionally, this has consisted of stopping the code on the adiabat when the temperature dropped to $1800 \mathrm{~K}$. The chemical composition is fixed at this point and the adiabat then resumes. Presently in CHEETAH, the freezing temperature is $2145 \mathrm{~K}$, as determined by the normalization of the parameters of the code. The difference is insignificant, given our present knowledge of the adiabat. However, the procedure of freezing is important and the two reasons are as follows:

1. Increased carbon monoxide. Calorimetric data taken by D. Ornellas always shows the presence of considerable CO. ${ }^{2}$ This is also present in the codes near $2000 \mathrm{~K}$ but the CO reacts to $\mathrm{CO}_{2}$ if chemical equlibrium is allowed to persist to near $1 \mathrm{~atm}$.

2. Effect on $E_{0}$. The total energy of detonation is determined following cut-off of the CHEQ calculation at $1 \mathrm{~atm}$, with the water remaining completely as gas. We find these results:

\begin{tabular}{llrrr} 
& Density & \multicolumn{3}{c}{$\mathrm{E}_{0}(\mathrm{~kJ} / \mathrm{cc})$} \\
\cline { 3 - 5 } & $(\mathrm{g} / \mathrm{cc})$ & Freeze & \multicolumn{1}{c}{ Eqm } & Meas \\
\hline BTF & 1.852 & 10.8 & 12.0 & 11.2 \\
FEFO & 1.607 & 8.8 & 9.3 & 8.3 \\
HNS & 1.655 & 7.6 & 8.3 & 7.5 \\
HMX & 1.893 & 11.1 & 11.9 & 11.0 \\
HNB & 1.965 & 13.9 & 13.9 & 13.7 \\
PETN & 1.763 & 10.6 & 11.0 & 10.3 \\
TATB & 1.83 & 7.8 & 8.3 & 7.3
\end{tabular}

The CHEQ calculations with freezing are considerably smaller than those run all the way at equilibrium and they agree with the calorimetrically measured energies of detonation listed in the previous report.

The reasons for the freeze are considered further in Chapter 10.

\section{The Reasons why CHEQ is Good at Large Volumes (by Al Nichols)}

We are going to use CHEQ to build the low-pressure third term of the JWL, so we hope it will be correct. It has been noted that the adiabats calculated in CHEQ go to the corrrect low density 
limit while those calculated by CHEETAH or TIGER do not. The question is why do we expect CHEQ to do this part of the problem correctly.

1. The heat capacity of each species in CHEQ has been fit by one of two algebraic functions. The heat capacities used in these fits come from a combination of theory and experimental data. The fitting ensures that the reference energy of each species is correct at the low density limit.

2. At its core, CHEQ uses intermolecular potentials to describe the interactions between species. In a real non-Coulombic system, the interactions between two molecules is short-range. Therefore, at low enough density, the collision cross-section between two molecules becomes small compared to the distance between them. Under such a circumstance, the fluid will behave as if it were an ideal gas.

3. Each fluid phase in CHEQ is mapped into an equivalent one-component fluid. The fluid has the same number of molecules as the original fluid and an average interaction potential which is determined by mixing rules from the intermolecular potentials. For a pure system, the onecomponent system is just the pure material.

4. The excess free energy of the effective one-component fluid is calculated by finding the hard sphere reference system which minimizes the excess free energy of the system. This methodology is called a variational perturbation theory because the term for the excess free energy is a perturbation expansion from the reference system. It is known that the excess free energy which is calculated by this procedure is an upper bound on the excess free energy of the actual system. Because the interaction between two hard spheres has a finite range, this kind of system goes to the ideal gas limit.

All the thermodynamic variables in CHEQ are expressed in terms of their difference from the ideal gas contribution. Furthermore, CHEQ uses variational perturbation theory to calculate the thermodynamic quantities for the single component reference fluid. The produces the best value of the free energy for the single component fluid.

Because CHEQ calculates corrections to the ideal gas and low temperature behavior of the material, when the state that is being calculated is going to lower density, CHEQ will automatically revert back to the ideal gas state. 


\section{Setting $\omega$ from CHEQ/What determines $\omega$}

We are going to use CHEQ to set the values of $\omega$ in the JWL. They are obtained by fitting pressure for $10>v>1 \mathrm{~atm}$ point with a power fit, as shown in Fig. 2-1. The power is $1+\omega$.

The derived $\omega$ 's are listed in Table $2-1$ with the $1800 \mathrm{~K}$ freeze included. The order of the $\omega$ values does not appear obvious. We shall here show generally why it occurs this way and show why a thermochemical code is needed to get it. In the previous report, we showed that, at constant volume,

$$
\omega=\mathrm{R} / \mathrm{C}_{\mathrm{V}}
$$

where $\mathrm{C}_{\mathrm{V}}$ is the heat capacity at constant volume. We, therfore, assume that $\omega$ is inversely proportional to the heat capacity, $\mathrm{C}_{\mathrm{a}}$, now described along the adiabat. We also assume that

$$
\mathrm{C}_{\mathrm{a}} \sim \mathrm{a}+\mathrm{bT}
$$

where we make it somewhat temperature-dependent. The variable $\omega$ averages over the heat capacity in the temperature range it covers. We write

$$
\omega=\frac{1}{\left\langle\mathrm{C}_{\mathrm{a}}\right\rangle}=\left(\mathrm{T}_{\mathrm{cj}}-\mathrm{T}_{\mathrm{e}}\right)\left[\int_{\mathrm{T}_{\mathrm{e}}}^{\mathrm{T}_{\mathrm{cj}}}(\mathrm{a}+\mathrm{bT}) \mathrm{dT}\right]^{-1},
$$

where $\left\langle\mathrm{C}_{\mathrm{a}}\right\rangle$ is the average heat capacity on the adiabat (different from $\mathrm{C}_{\mathrm{v}}$ and $\mathrm{C}_{\mathrm{p}}$ ), $\mathrm{T}_{\mathrm{cj}}$ is the temperature at the $\mathrm{C}-\mathrm{J}$ point and $\mathrm{T}_{e}$ is the temperature at the end of the adiabat, which is at $1 \mathrm{~atm}$ if the code can take it that far. We assume that the products are unchanged from start to finish, integrate Eq. 11 and cancel $\left(\mathrm{T}_{\mathrm{cj}}-\mathrm{T}_{\mathrm{e}}\right)$. We have

$$
\omega=\frac{1}{a+\frac{b}{2}\left(T_{c j}+T_{e}\right)}
$$

This says that the higher the C-J temperature (which probably also raises $T_{e}$ ), the smaller is $\omega$. 
Five extreme explosives have been selected in Table 2-2. The first two are HNB and TNM. HNB has a perfect two oxygens per carbon and nothing else but nitrogen, so that the products can only be $\mathrm{CO}_{2}$ and $\mathrm{N}_{2}$. TNM has an overabundance of oxygen so that the carbon burns to $\mathrm{CO}_{2}$ and excess $\mathrm{O}_{2}$ is released. Again, only nitrogen makes up the rest. HNB has one the highest C-J temperatures and TNM one of the lowest, with a factor of 2.5 difference. Neither makes any solid carbon. We expect $\omega$ for TNM to be this much larger than that of $\mathrm{HNB}$, with there being no difference between the equilibrium and freeze runs.

The results are listed in Table 2-2, with the temperatures coming from CHEQ and the compositions from CHEETAH. The compositions are generally similar from both codes but the C-J temperatures in CHEETAH BKWC ${ }^{3}$ are usually somewhat lower than those of $\mathrm{CHEQ}$. What we really need from CHEQ are the 1 atmosphere temperatures, which are only roughly obtained from CHEETAH. We see that these temperatures can be below room temperature. This is what the code predicts with no heat flow in from the surroundings.

There is another difference between HNB and TNM. The former has $63 \%$ polyatomic product molecules and the TNM only $17 \%$. The HNB has a larger heat capacity because the larger molecules have more degrees of freedom to store it in. This reduces the difference in $\omega$ to about 2.

The other examples all have carbon which can burn partially. The codes have carbon creating solid and $\mathrm{CO}_{2}$ at the C-J point. These recombine to form maximum $\mathrm{CO}$ at the freeze point. If freezing does not occur, the $\mathrm{CO}$ reverts back to carbon and $\mathrm{CO}_{2}$ at 1 atmosphere. In $\mathrm{CHEQ}$ and CHEETAH, the carbon never precipitates and is always ready to back-react. The effect of the freeze is to halt the reaction with less polyatomic product (smaller $C_{a}$ ). This decreases the 1 atmosphere temperature for the frozen products. With the same energy and less heat capacity, the temperature distance to be traveled is longer. Once again the issue of freezing has a big effect on the EOS.

\section{Infinite-Volume Energy in the JWL}

Most energies in JWL's have been selected to agree with the measured heat of detonation at 1 atmosphere. One can refine this to the energy of detonation, but this is still not right. The energy should be that taken out to infinite volume. This will add about $0.5 \mathrm{~kJ} / \mathrm{cc}$ to the 1-atmosphere $E_{0}$ values, an important correction. We shall consider the reason why this should be done. From Eq. 18 in Chapter 1, we have the JWL adiabat energy

$$
E_{S}=\left(A / R_{1}\right) \exp \left(-R_{1} v\right)+\left(B / R_{2}\right) \exp \left(-R_{2} v\right)+C / \omega v \omega
$$


Eq. 13 has this form only as long as one limit is $v$ and the other is $\infty$. If 1 atm were the limit, then three small but finite terms would appear. We can get the infinite volume energy using the Look-Up Table function

$$
\mathbf{f}=P_{\mathrm{S}} \mathrm{v} / \mathrm{E}_{\mathrm{S}}
$$

At large volumes, we can equate Eq. 14 with the third term of the JWL. As previously shown, as the pressure approaches zero

$$
\text { f } \rightarrow \omega=\Gamma-1 \text {. }
$$

In CHEQ, we obtain internal energy as the result of the calculation. This can be turned into an approximate energy of detonation by adding an energy (not the heat) of formation. We can then add a constant of integration until we get the proper adiabat energy. We already know what $\omega$ is from the fit of $P_{S}$ versus $v$ from $v>10$ on. We now change the added energy until $f=\omega$ agrees with the value we expect. Now we can read off both $\mathrm{E}_{0}(1 \mathrm{~atm})$ and $\mathrm{E}_{0}(\infty)$.

\section{Fixing $\mathbf{R}_{1}$}

The second part of JWL construction occurs in the first term, where we seek to determine $R_{1}$. We recall that the Lennard-Jones hard-core potential energy goes as $r^{-12} \sim v^{-4}$. Then, 4

$$
\mathrm{P} \sim \partial \mathrm{U} / \mathrm{r}=\mathrm{v}^{-4.33}
$$

Now, let us consider volumes from $0.75<v<1.5$, ie. from the detonation point until the A-term of the JWL is down $95 \%$ in pressure. Empirically, we find that

$$
\mathrm{v}^{-\mathrm{n}} \sim 100 \exp (-\mathrm{nv}) \quad 4<\mathrm{n}<5
$$

We, therefore, select

$$
R_{1} \approx 4.5 \text {. }
$$

We expect $R_{1}$ to be a constant for all explosives providing temperature is not folded into it. Harold Zimmerman notes that the JWL is a Gruneison EOS of the form 


$$
P_{S}=M(v)+N(v) E
$$

so that temperature will appear in the energy term. The $0 \mathrm{~K}$ cold compression is the first term, which includes the $R_{1}$ exponential. Thus, we do not expect $R_{1}$ to change from one explosive to another because it is the $0 \mathrm{~K}$ adiabat. If we consider an explosive Hugoniot up to $\mathrm{P} \sim 80 \mathrm{GPa}$, we find that $R_{1}$ needs to be about 6 to account for the higher pressure created by temperature and entropy.

Larry Fried notes that the constant- $R_{1}$ assumption is true only for spherical molecules. CHEQ presently takes product of all shapes and "spherizes" them. In reality, the variation of shape and composition should cause $R_{1}$ to be somewhat different.

\section{JWL-Changing with Density}

It is common for samples to have slight density differences. We need a general process of converting JWL's as a function of density, a process that leads us to expecting certain relationships between variables. Table 2-3 shows the density relationships for several variables. ${ }^{2,5-7}$ The oxygen balance is defined as being 8,9

$$
\text { oxygen balance }=\left[\frac{2 \mathrm{O}+\mathrm{F}+\mathrm{Cl}}{2 \mathrm{C}+\mathrm{H}}\right]
$$

where the oxidizers are in the numerator and the fuel in the denominator. The bigger the ratio, the more oxygen there is. A ratio of 1.0 gives a fully oxidized explosive. In Table 2-3, we see that the power of the density is 1.0 only for the over-oxidized TNM. Otherwise, the power increases slightly as the oxygen balance decreases: 1.1 for PETN, 1.2 for HMX and 1.4 for TATB. The thermodynamic reason for this is shown for TATB in Table 2-4. As the density decreases, so does the efficiency of burning. More $\mathrm{CO}$ is formed and less $\mathrm{CO}_{2}$. Because most explosives are underoxidized, we take as an general relation

$$
E_{0} \sim \rho_{0}^{4 / 3}
$$

Using Eqs. 10 and 12 from Chapter 1, we get

$$
\mathrm{D} \sim \mathrm{E}_{\mathrm{o}}^{1 / 2} \sim \rho_{\mathrm{o}}^{2 / 3}
$$


and

$$
P_{j}=\rho_{o} D^{2}\left(1-v_{j}\right) \approx \rho_{o}^{7 / 3}
$$

The final thing we need is the power for the cylinder energies of detonation, $\mathbf{E}_{\mathbf{d}}$. From the cylinder data, we get these powers:

\begin{tabular}{lcccc} 
& Density & \multicolumn{3}{c}{ Powers at these Volumes } \\
\cline { 3 - 5 } & $(\mathrm{g} / \mathrm{cc})$ & 2.2 & 4.1 & 6.5 \\
\hline HNS & 1.0 to 1.68 & 1.66 & 1.53 & 1.50 \\
PETN & 1.27 to 1.77 & 1.83 & 1.65 & 1.51 \\
HMX & 1.19 to 1.89 & 1.47 & 1.40 & 1.32
\end{tabular}

We, therefore, generalize to

$$
\mathrm{E}_{\mathrm{d}}(2<\mathrm{v}<7) \sim \rho_{0}{ }^{3 / 2} .
$$

\section{References}

1. P. A. Urtiew and B. Hayes, Empirical Estimate of Detonation Parameters in Condensed Explosives, Lawrence Livermore National Laboratory Report UCRL-JC-103219 (1990).

2. D. L. Ornellas, Calorimetric Determinations of the Heat and Products of Detonation for Explosives: October 1961 to April 1982, LLNL Report UCRL-52821 (1982).

3. We consider the old CHEETAH and TIGER BKWR to be totally obsolete and it will not be used in this report. The difficulties of BKWC were considered in P. C. Souers and L. C. Haselman, Jr., Detonation Equation of State at LLNL, 1993, LLNL Report UCRL-ID-116113 (March, 1994).

4. G. C. Maitland, M. Rigby, E. B. Smith and W. A. Wakeham, Intermolecular Forces (Clarendon Press, Oxford, 1981), pp. 29-31.

5. D. J. Steinberg, "Comparison of Experimental Data on Detonation Velocity and Chapman-Jouget Pressure vs Initial HE Density with Predictions from Ree's Model Equation of State," Proceedings Eighth Symposium (International) on Detonation, Albuquerque, NM, July 15-19, 1985, p. 513.

6. D. Price, The Detonation Velocity - Loading Density Relation for Selected Explosives and Mixtures of Explosives, Naval Surface Weapons Center Report NSWC TR 82-298 (1982). 
7. N. L. Coleburn, B. E. Drimmer, and T. P. Liddiard, Jr., "The Detonation Properties of DATB (1, 3-Diamino, 2, 4, 6-Trinitrobenzene," Third Symposium on Detonation. Princeton University, September $26-28,1960$, p. 761.

8. M. J. Kamlet and H. G. Adolph, Propellants Explosives \& Pyrotechnics $\underline{4} 30$ (1979).

9. S. R. Jain, Propellants Explosives \& Pyrotechnics 12188 (1987).

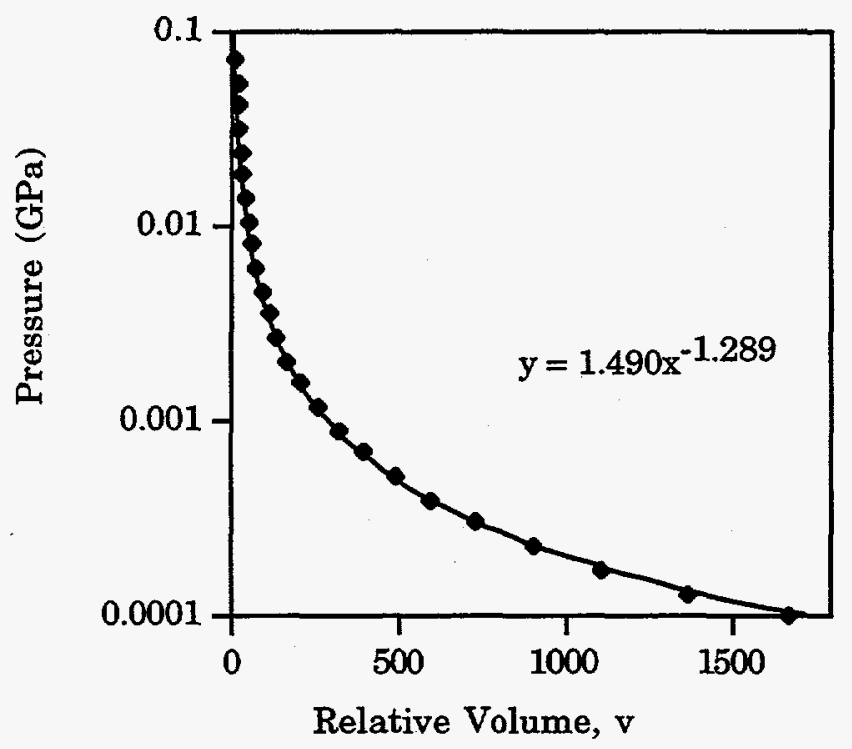

Fig. 2-1. Adiabat pressure versus relative volume for $1.763 \mathrm{~g} / \mathrm{cc}$ PETN with an $1800 \mathrm{~K}$ freeze as calculated from CHEQ. The smallest volume used is just above 10 so that the pressure curve describes only the third term of the JWL. The fit from $10<\mathrm{v}$ about 2000 is good. 
Table 2-1. CHEQ-calculated third-term JWL parameters for various explosives. $E_{0}$ is the 1 atm energy of detonation; $E_{0}(\infty)$ is the energy at infinite volume. An $1800 \mathrm{~K}$ freeze was used.

\begin{tabular}{|c|c|c|c|c|c|c|c|c|}
\hline & $\begin{array}{c}\text { Main } \\
\text { ingred- } \\
\text { ient }\end{array}$ & $\begin{array}{c}\text { Density } \\
(\mathrm{g} / \mathrm{cc})\end{array}$ & $\omega$ & $\begin{array}{c}\mathrm{E}_{\mathbf{0}}(\infty) \\
(\mathrm{kJ} / \mathrm{cc})\end{array}$ & $\begin{array}{c}\mathbf{E}_{o} \\
(\mathrm{~kJ} / \mathrm{cc})\end{array}$ & $\begin{array}{c}\text { diff } \\
(\mathrm{kJ} / \mathrm{cc})\end{array}$ & $\begin{array}{c}\text { CHEQ } \\
\mathrm{T}_{\mathrm{cj}} \\
(\mathrm{K})\end{array}$ & $\begin{array}{c}\mathrm{CHEQ} \\
\mathrm{P}_{\mathrm{cj}} \\
(\mathrm{GPa})\end{array}$ \\
\hline TNM & & 1.65 & 0.37 & 3.7 & 3.6 & 0.1 & 2506 & 15.1 \\
\hline RDX & & 1.72 & 0.35 & 10.2 & 9.8 & 0.3 & 4266 & 32.2 \\
\hline RX-39-AB & $\varepsilon \mathrm{CL}-20$ & 1.94 & 0.34 & 11.8 & 11.4 & 0.3 & 4333 & 40.9 \\
\hline HMX & & 1.89 & 0.34 & 11.4 & 11.1 & 0.3 & 4131 & 42.5 \\
\hline NM & & 1.13 & 0.34 & 5.7 & 5.4 & 0.3 & 3729 & 12.3 \\
\hline BTF & & 1.85 & 0.33 & 11.2 & 10.8 & 0.4 & 4714 & 32.2 \\
\hline LX-14 & HMX & 1.83 & 0.33 & 10.6 & 10.3 & 0.4 & 3997 & 34.7 \\
\hline $\mathrm{RX}-41-\mathrm{AB}$ & $\mathrm{K}-6$ & 1.86 & 0.32 & 10.7 & 10.3 & 0.4 & 4208 & 36.1 \\
\hline RX-23-AA & hyd nit & 1.42 & 0.32 & 7.6 & 7.0 & 0.5 & 3126 & 26.6 \\
\hline FEFO & & 1.61 & 0.31 & 9.3 & 8.8 & 0.5 & 4584 & 30.0 \\
\hline HNS & & 1.00 & 0.31 & 4.0 & 3.7 & 0.3 & 3656 & 7.1 \\
\hline $\mathrm{RX}-45-\mathrm{AA}$ & ANTA & 1.75 & 0.30 & 6.9 & 6.6 & 0.4 & 3192 & 27.4 \\
\hline NQ & & 1.69 & 0.30 & 7.1 & 6.7 & 0.4 & 2884 & 24.8 \\
\hline PETN & & 1.76 & 0.29 & 11.2 & 10.6 & 0.6 & 4376 & 32.0 \\
\hline $\mathrm{RX}-52-\mathrm{AE}$ & TATB & 1.78 & 0.29 & 8.5 & 8.0 & 0.5 & 3759 & 25.7 \\
\hline $\mathrm{RX}-48-\mathrm{AA}$ & ADNBF & 1.85 & 0.28 & 9.5 & 9.0 & 0.5 & 4144 & 30.1 \\
\hline $\mathrm{RX}-47-\mathrm{AA}$ & CL-14 & 1.82 & 0.28 & 8.8 & 8.3 & 0.5 & 3890 & 27.7 \\
\hline HNS & & 1.66 & 0.27 & 8.1 & 7.6 & 0.6 & 4052 & 20.2 \\
\hline LX-17 & TATB & 1.91 & 0.26 & 8.6 & 8.1 & 0.5 & 3284 & 28.0 \\
\hline TNT & & 1.63 & 0.25 & 7.8 & 7.2 & 0.6 & 3888 & 20.4 \\
\hline PETN & & 1.26 & 0.25 & 8.1 & 7.3 & 0.8 & 4631 & 14.0 \\
\hline TATB & & 1.83 & 0.25 & 8.4 & 7.8 & 0.6 & 3365 & 25.8 \\
\hline HNB & & 1.97 & 0.22 & 15.2 & 13.9 & 1.3 & 5116 & 39.9 \\
\hline
\end{tabular}


Table 2-2. Properties of five explosives with extreme values of $\omega$. "Eqm" is equilibrium. The temperatures $T_{c j}$ and $T_{e}$ are at the C-J point and at the 1 atmosphere end of the adiabat.

\begin{tabular}{ccc|ccc|} 
& $\omega$ & $\omega$ & Temp. (K) from CHEQ \\
\cline { 2 - 6 } $\begin{array}{c}\text { Explo- } \\
\text { sive }\end{array}$ & $\begin{array}{c}\text { Equi- } \\
\text { librium }\end{array}$ & $\begin{array}{c}2145 \mathrm{~K} \\
\text { Freeze }\end{array}$ & $\mathrm{C}-\mathrm{J}$ & $\mathrm{Eqm}$ & freeze \\
\hline HNB & 0.21 & 0.21 & 5116 & 664 & 663 \\
TNM & 0.37 & 0.36 & 2275 & 120 & 120 \\
& & & & & \\
BTF & 0.12 & 0.33 & 4714 & 817 & 257 \\
RDX & 0.20 & 0.35 & 4266 & 443 & 206 \\
TATB & 0.18 & 0.25 & 3365 & 438 & 284
\end{tabular}

\begin{tabular}{|c|c|c|c|c|c|c|c|c|}
\hline \multirow{2}{*}{$\begin{array}{l}\text { Explo- } \\
\text { sive }\end{array}$} & & \multicolumn{6}{|c|}{ mols/mol CHEETAH } & \multirow{2}{*}{$\begin{array}{c}\text { fraction } \\
\text { polyat- } \\
\text { omic }\end{array}$} \\
\hline & & $\mathrm{H}_{2} \mathrm{O}$ & $\mathrm{N}_{2}$ & $\mathrm{CO}_{2}$ & $\mathrm{CO}$ & $\mathrm{C}$ & Total & \\
\hline \multirow[t]{3}{*}{ HNB } & $\mathrm{CJ}$ & 0.1 & 3.0 & 5.7 & 0.3 & $\overline{0.0}$ & $\overline{9.1}$ & 0.63 \\
\hline & Freeze & 0.0 & 3.0 & 6.0 & 0.0 & 0.0 & 9.0 & 0.66 \\
\hline & Eqm End & 0.0 & 3.0 & 6.0 & 0.0 & 0.0 & 9.0 & 0.67 \\
\hline \multirow[t]{4}{*}{ TNM } & CJ & 3.0 & 2.0 & 1.0 & 0.0 & $\overline{0.0}$ & 6.0 & 0.17 \\
\hline & Freeze & 3.0 & 2.0 & 1.0 & 0.0 & 0.0 & 6.0 & 0.17 \\
\hline & Eqm End & 3.0 & 2.0 & 1.0 & 0.0 & 0.0 & 6.0 & 0.17 \\
\hline & & $\mathrm{H}_{2} \mathrm{O}$ & $\mathrm{N}_{2}$ & $\mathrm{CO}_{2}$ & $\mathrm{CO}$ & $\mathrm{C}$ & Total & \\
\hline \multirow[t]{3}{*}{ BTF } & CJ & & 3.0 & 2.6 & 0.7 & $\overline{2.6}$ & 9.0 & 0.29 \\
\hline & Freeze & & 3.0 & 1.3 & 3.5 & 1.3 & 9.0 & 0.14 \\
\hline & Eqm End & & 3.0 & 2.9 & 0.3 & 2.9 & 9.0 & 0.32 \\
\hline \multirow[t]{3}{*}{ RDX } & CJ & 3.0 & 3.0 & 1.3 & 0.4 & 1.3 & 9.0 & 0.48 \\
\hline & Freeze & 2.6 & 3.0 & 0.9 & 1.5 & 3.6 & 11.9 & 0.29 \\
\hline & Eqm End & 2.7 & 3.0 & 1.6 & 0.0 & 1.2 & 8.7 & 0.50 \\
\hline \multirow[t]{3}{*}{ TATB } & CJ & 3.0 & 3.0 & 1.4 & 0.2 & 4.4 & 12.0 & 0.37 \\
\hline & Freeze & 2.9 & 3.0 & 1.2 & 0.7 & 4.1 & 11.9 & 0.34 \\
\hline & Eqm End & 2.8 & 3.0 & 1.6 & 0.1 & 4.3 & 11.8 & 0.37 \\
\hline
\end{tabular}


Table 2-3. Density dependence of various parameters as a function of oxygen balance.

\begin{tabular}{cccccccc} 
& \multicolumn{3}{c}{$\begin{array}{c}\text { No. of } \\
\text { Explo- }\end{array}$} & Oxygen & Data & \multicolumn{5}{c}{ Power of the Density } & & \\
\cline { 4 - 7 } sive & Balance & Points & $\mathrm{D}$ & $\mathrm{E}_{\mathrm{o}}$ & $\mathrm{P}_{\mathrm{j}}$ & $1-\mathrm{v}_{\mathbf{j}}$ & Source \\
\hline TNM & 8.00 & & 0.80 & 1.0 & 2.2 & -0.36 & CHEETAH \\
HNB & 2.00 & & 0.78 & 1.1 & 2.3 & -0.29 & CHEETAH \\
PETN & 1.33 & 3 & 0.68 & 1.2 & 2.0 & -0.33 & Cyl JWL \\
PETN & 1.33 & 2 & & 1.0 & & & 2 \\
PETN & 1.33 & 11 & 0.76 & & & & 6 \\
PETN & 1.33 & & 0.74 & 1.1 & 2.3 & -0.18 & CHEETAH \\
HMX & 1.00 & 2 & 0.66 & 1.2 & 2.1 & -0.26 & Cyl JWL \\
HMX & 1.00 & 2 & & 1.3 & & & 2 \\
HMX & 1.00 & 31 & 0.66 & & & & 5 \\
HMX & 1.00 & & 0.65 & 1.2 & 2.1 & -0.19 & CHEETAH \\
RDX & 1.00 & 25 & 0.65 & & & & 5 \\
DATB & 0.71 & 11 & 0.60 & & & & 7 \\
HNS & 0.71 & 8 & 0.65 & 1.4 & 2.2 & -0.11 & Cyl JWL \\
HNS & 0.71 & 2 & & 1.5 & & & 2 \\
HNS & 0.71 & & 0.61 & 1.3 & 2.1 & -0.14 & CHEETAH \\
TATB & 0.67 & & 0.74 & 1.4 & 2.3 & -0.16 & CHEETAH \\
Tetryl & 0.63 & 13 & 0.61 & & & & 6
\end{tabular}

Table 2-4. Change of carbon composition with density for TATB as calculated by CHEETAH.

\begin{tabular}{cc|cccc}
\multirow{2}{*}{$\begin{array}{c}\text { Density } \\
\text { (g/cc) }\end{array}$} & $\begin{array}{c}\text { C-J } \\
\text { Pressure }\end{array}$ & \multicolumn{4}{|c}{ Composition (mols/mol) } \\
\cline { 3 - 6 }$(\mathrm{GPa})$ & $\mathrm{H}_{2} \mathrm{O}$ & $\mathrm{CO}_{2}$ & $\mathrm{CO}$ & $\mathrm{C}$ \\
\hline 1.9 & 29.2 & 3.0 & 1.3 & 0.4 & 4.3 \\
1.8 & 25.0 & 3.0 & 1.4 & 0.2 & 4.4 \\
1.6 & 18.3 & 3.0 & 1.3 & 0.5 & 4.3 \\
1.4 & 13.2 & 3.0 & 1.0 & 0.9 & 4.0 \\
1.2 & 9.5 & 2.9 & 1.5 & 0.8 & 3.6 \\
1.0 & 6.7 & 2.6 & 0.6 & 2.2 & 3.0
\end{tabular}




\section{Chapter 3. Cylinder JWL Fits}

\section{The Code and JWL-Making}

The cylinder test has been used for three decades at LLNL and is the heart of the explosive performance data base. ${ }^{1}$ A schematic of the cylinder is shown in Fig. 3-1 for a 1-inch diameter size. For a half-wall cylinder, this is a $12.72 \mathrm{~mm}$ radius and a $1.36 \mathrm{~mm}$ wall thickness. The full-wall thickness is twice as great. The cylinder is $300 \mathrm{~mm}$ long, with the observation position $210 \mathrm{~mm}$ out. The Fabry angle is $7^{\circ}$ at this position. It is initiated with a plane-wave lens, whose detonation front in the inner half is, according to Larry Shaw, about \pm 40 to $\pm 60 \mathrm{~ns}$ wide. It is usually assumed, without confirmation, that the detonation wave has smoothed itself out by the time the wall measurment is made.

The cylinder code in HEMP was designed by Bill Quirk. The copper is 24 zones thick. In a halfwall cylinder, the initial zone size is $0.2 \mathrm{~mm}$ along the wall length and $0.0567 \mathrm{~mm}$ width for an aspect ratio of 3.5 and 88 zones $/ \mathrm{mm}^{2}$. As the problem progresses, the zones become ever thinner. The explosive has lines added and deleted. Just before detonation, which can be $20 \mu$ after the problem begins, there are 48 zones in the explosive from the axis to the inner wall. The zone dimensions are $0.40 \times 0.265 \mathrm{~mm}$ for an aspect ratio of 1.5 and 9.4 zones $/ \mathrm{mm}^{2}$. As compression narrows the zones, a line is removed to change the aspect ratio to about 0.7 with 4 zones $/ \mathrm{mm}^{2}$. A type- 1 slideline runs between the metal and the explosive; both ends are stonewalled and a line detonation is used with the combination beta/program burn. The copper has the Steinberg-Guinan metal model with the principal stress spall model. The cutoff pressure is taken from Steinberg and is not changed. ${ }^{2}$ The CPU time is about 90 minutes per run.

The timing between the code run and the averaged data is never known absolutely and we use these rules. For velocities, the two curves are fitted together in the middle of the vertical rise between the jump-off and the second bump. The streak distances $\left(R-R_{0}\right)$ are fitted by bringing them together at time zero. This requires noting where the change of slope is for the code run and moving the curve both in magnitude and time until the distance starts to first increase at zero. The streak camera starts at time zero or the data cannot be used. Even though the timing may be reset to zero, the magnitude of the distance covered cannot be touched. 
We now wish to generate JWL's which will be compared with cylinder data using the HEMP code. Because of the constraints on the variables, the process will be different than it was for the old 3-point cylinder method. There, only the density $\rho_{0}$ and the detonation energies at $v=2.2,4.1$ and 6.5 were fixed. We then guessed $R_{1}, R_{2}, \omega$ and $P_{j}$ and calculated $A, B$ and $C$ with three linear equations. We were willing to adjust 7 variables in order to get the linear equations to work. The result gave self-consistent numbers because all the equations had been folded in. We also assumed the data, ie. the detonation energies, were perfect and changed constants to get the best agreement. This overreliance on the data led to JWL's with vastly different constants, because a small error in the data could be greatly magnified by the linear equation solution process.

Here we use a different procedure, one in which the constants are overspecified and which really works only for near-ideal explosives. We will have to check that the detonation velocities and pressures are consistent when calculated by different routes. Fig. 3-2 shows this process. The third, low-pressure term with $\omega$ and $E_{0}(\infty)$ is built entirely from a thermochemical code. Then $R_{1}$ and $R_{2}$ are fixed at 4.5 and 1.5, $\Gamma_{j}+1$ is set to some value and $A$ and $B$ are estimated. $A$ is expected to have a certain relation to $\rho_{0} D^{2}$ and $B$ is some factor smaller than $A$. The energies of detonation, $E_{d}$, are calculated and compared to the expected values obtained by use of the standard. Both $\mathrm{D}$ and $\mathrm{P}_{\mathrm{j}}$ are calculated and are brought into agreement by two different paths of calculation. We require that $D$ and $\mathrm{P}_{\mathrm{j}}$ agree to less than $0.005 \%$ whereas $\mathrm{E}_{\mathrm{d}}$ need only agree to with a few $\%$. Thus is the uncertainty of the data folded into this approach. If the set does not match, we change bhe and repeat until agreement is reached.

\section{JWL Cylinder Results (with Stella McGuire)}

The JWL's used in this study are listed in Table 3-1. Below the constants are the \% agreement of the code as compared with the data. Two points are listed for simplicity: the jump-off velocity as compared with Fabry data, if any, and the last measured streak camera $R$ - $R_{0}$ distance point where the run went beyond $5 \mu \mathrm{s}$. So this shows with two numbers the fit at short and at long times. The cylinder test is based on long-time data and we can only expect this to be handled accurately, with $\pm 1 \%$ being obtainable for all long-time points. Jump-off can vary by $\pm 15 \%$ with ease; this value declines as one proceeds to the later bumps. The streak camera distance is used because this is the measured value, whose accuracy improves as time increases. The differentiated streak camera data is not used because this creates a "curtain" of noise about \pm 3 to $4 \%$ wide.

Some of the explosives in Table 3-1 are mixtures. Their prime ingredients are: LX-14 (HMX), LX-17 (TATB), AFX-902 (NQ, nitroguanidine), RX-26-AF (HMX-TATB), RX-39-AB (ECL-20), 
RX-45-AA (ANTA), RX-47-AA (CL-14), RX-48-AA (ADNBF) and RX-52-AE (TATB-FEFO). More details are given in Appendix A. The CO Look-Up Table needed for LX-14 is listed in Table 3-2.

We wish to note: because the low pressure end of the adiabat is built up with CHEQ, the $R_{1}$ $=4.5 \mathrm{JWL}$ 's may be used for large volume far-field blast problems. They are not restricted to $\mathrm{v}<10$ problems!

The $1.76 \mathrm{~g} / \mathrm{cc}$ PETN "standard" is shown in Fig. 3-3a. The old standard with $\mathrm{A}=10.32$ is shown for Fabry velocity by curve $\mathrm{C}$ (dashed) and the streak camera distance by curve $\mathrm{G}$ (dotted). Neither curve is good. The new $A=6.17$ standard is shown for Fabry velocity by curve $B$ (solid) and the streak distance by curve A (solid). Both are excellent fits. The new JWL method clearly produces a better EOS and the percent-plot shows off the differences with great accuracy. LX-14 is in Fig. 3$3 \mathrm{~b}$. Although we consider the $\mathrm{A}=7.65 \mathrm{JWL}$ to be essentially the well-known $381 \mathrm{JWL}$, the former does fit better for streak camera distance at an earlier time. Also shown is the result of the CO Look-Up Table. All of the Fabrys look like the one plotted for the A=7.65 JWL. Fig. 3-3d shows RX41- $\mathrm{AB}$ with typical values for a more modern shot with a longer and more accurate Fabry.

LX-17, shown in Fig. 3-3c, is so non-ideal that the JWL system does not work. The $A=6.92$ JWL obtained by this chapter's method is not as good as the old $A=4.603 \mathrm{JWL}$ where $R_{1}$ and $R_{2}$ are different. There is no way to set $R_{1}=4.5$ and $R_{2}=1.5$ and get good agreement. We recall in Chapter 1 that only a spike pressure of about $36 \mathrm{Gpa}$ exists, yet we have a $30 \mathrm{GPa}$ detonation pressure in the $A=4.603 \mathrm{JWL}$. Is it not possible to raise the pressure to what it really is and reset the JWL? The answer is no, because we must first match up the slopes at the detonation point

$$
\left(\frac{\partial P}{\partial v}\right)_{j}=-R_{1} \operatorname{Aexp}\left(-R_{1} v_{j}\right)=-\rho_{0} D^{2}
$$

These are hard to match up, because one is constant and the other changes rapidly with volume. The smaller the detonation volume, the harder the match is. Moreover, the EOS must also fit to the three cylinder energies. So the detonation pressure cannot be cranked up to add non-ideal effects to a JWL. The actual C-J pressure calculated for LX-17 is $27 \mathrm{GPa}$; the use of $30 \mathrm{GPa}$ in the A $=4.603 \mathrm{JWL}$ represents the maximum pressure that was squeezed out of the JWL form. This means that a JWL detonation point is achieved to fit the math and this point cannot be taken as more than an approximation to the detonation C-J point. 
When we calculate the cylinder energies using the $A=4.603 \mathrm{LX}-17 \mathrm{JWL}$, we find that they are 9,7 and 5\% larger than expected at $\mathrm{v}=2.2,4.1$ and 6.5. "Expected", of course, is a linear relationship with the standard PETN. All this means is that LX-17, with its time-dependent burn, cannot be simply related to PETN and is not derivable from the $R_{1}=4.5$ system. The only way to get a JWL is from hydrocode runs and these are empirical fits only to those experiments.

Assuming we try the new JWL-making system, what can we find for a trend? We expect $A$ to be proportional to pressure, ie to

$$
A \sim \rho_{0} D^{2}(1-v)
$$

The coefficients from Table 3-1 are plotted in Fig. 3-4. The powers of A, B and C are 1.2, 0.75 and 0.35 . So A may well be directly proportional had the process of picking coefficients been set that way. $B$ and $C$ are certainly not proportional. Urtiew and Hayes tried a universal JWL fit to $\rho_{0} D^{2.3}$ now we see that all the coefficients cannot fit this requirement.

The detonation pressures in the Table 3-1 JWL's are higher than the C-J pressures of CHEETAH by $7 \pm 7 \%$. For CHEQ, it is $4 \pm 6 \%$. This does not mean very much. The JWL tends to predict high pressures because of the steepness of the A-term. Also, our process probably raised the pressures as well. The question of whether thermochemical codes calculate the true detonation pressure is still unanswered.

\section{The Detonation Energy Standard}

Each JWL that is validated against a cylinder shot is a legitimate standard, although some have better quality measurements than others. Some don't fit very well with the JWL function like LX-17, NM, low density HNS and especially TNM. With each JWL, we can calculate detonation energies at $v=2.2,4.1$ and 6.5 with each of the three paths. At each volume, we require that

$$
\frac{E_{d}}{E_{d}(\text { PETN })}=\left[\frac{u}{u(P E T N)}\right]^{1 / 2},
$$

where $u$ and uPETN are the cylinder velocities of explosive $x$ and the PETN standard and simularly for the detonation energy at some specified volume. This allows us to convert the results of many JWL's into a PETN standard energy. By averaging, we can get a better result than if we had relied only on the one PETN shot. Put another way, we really don't know the absolute values of $E_{d}$. These 
are also adjusted so that the group of cylinder shots have the best overall agreement. We get these results.

\begin{tabular}{cccc} 
& \multicolumn{3}{c}{ Detonation Energies $(\mathrm{kJ} / \mathrm{cc})$} \\
\cline { 2 - 4 } PETN & $\mathrm{v}=2.2$ & $\mathrm{v}=4.1$ & $\mathrm{v}=6.5$ \\
\cline { 2 - 4 } Average all & 0.0650 & 0.0775 & 0.0831 \\
\% stdev all & 0.0658 & 0.0775 & 0.0823 \\
& 1.9 & 0.9 & 1.7
\end{tabular}

The energies for PETN are those that match its specific JWL. All 14 runs from Table 3-1 are averaged into the bold-faced result, and these are the detonation energies we use as the standard at the cylinder positions of $R-R_{0}$ of $6,12.5$ and $19 \mathrm{~mm}$. The standard deviation of $\pm 2 \%$ at each volume, which is precision, not accuracy. We had previously guessed our knowledge to be $\pm 5 \%$.

The assumption that detonation energy has the same proportion to velocity-squared leads to an important conclusion: that all explosives have the same efficiency at the same volume. We define the efficiency, e, as the energy imparted to the copper wall divided by the detonation energy, both at the same volume. The volume of copper is $(1 / 2) \rho_{m} \pi\left[\left(R_{0}+W_{0}\right)^{2}-R_{0}^{2}\right]$ hu ${ }^{2}$, where $R_{0}$ is the initial inner radius, $W_{0}$ the initial wall thickness, $h$ the height and $u$ the measured velocity, which is considered to be the same at every point in the metal. The energy in the explosive is $\pi R_{0}{ }^{2} E_{d}$. The efficiency ratio, converted to percent, is

$$
e=50 \frac{\rho_{m}}{E_{d}}\left[\left(1+\frac{W_{0}}{R_{0}}\right)^{2}-1\right] \mathfrak{u}^{2}
$$

The calculated results are as follows:

\begin{tabular}{ccccc} 
& & \multicolumn{3}{c}{ \% Efficiency } \\
\cline { 4 - 5 } metal & wall & $\mathrm{e}(2.2)$ & $\mathrm{e}(4.1)$ & $\mathrm{e}(6.5)$ \\
\hline $\mathrm{Cu}$ & half & 59.3 & 59.5 & 59.8 \\
$\mathrm{Ta}$ & half & 66.1 & 66.9 & 67.1 \\
$\mathrm{Cu}$ & full & 74.1 & 75.0 & 75.2
\end{tabular}

We recall that the half-wall cylinder has $\mathrm{W}_{O} / \mathrm{R}_{0}=0.10$ and the full-wall 0.20 . The full-wall, with more confinement, is more efficient. The tantalum, which has a higher density and thereby creates 
more confinement. Also, the efficiency is set at a volume of 2.2. The assumption that the velocity represents the rigid body is probably good by the volume of 2.2 .

Detailed cylinder data are needed to give our JWL's a real pedigree. This data, listed in Table 3-3, is also needed to extend to generating other JWL's. From this, we generate the JWL's of Table 3-4, for which no code checks with data have been run. The cylinder data for HNS is from Pantex 4 and that for TNAZ from ARDEC. 5

\section{Problem: Changing the Density of LX-14}

Most JWL's do not come from a laborious comparison of data with a hydrocode run. They are whomped up on a moment's notice with whatever guessing material is available. The advantage of the systematic JWL's presented here is that they can better used for estimating. The easiest switch is a slight change of density, here illustrated in switching from $1.825 \mathrm{~g} / \mathrm{cc} \mathrm{LX}-14$ to $1.80 \mathrm{~g} / \mathrm{cc}$.

Previously, we might have thought these were so close that we don't have to bother. Now we can find out.

The density ratio is $1.80 / 1.825=0.9863$. We next use the relations of Chapter 2 to estimate:

$$
\begin{aligned}
& \mathrm{D} \sim(0.9863)^{0.667}(0.8783)=0.8703 \mathrm{~cm} / \mathrm{s} \\
& \mathrm{E}_{0}(\infty) \sim(0.9863)^{1.2}(0.1064)=0.1047 \mathrm{Mbar} \cdot \mathrm{cc} / \mathrm{cc} \\
& \mathrm{E}_{\mathrm{d}}(\mathrm{v}=2.2) \sim(0.9863)^{1.5}(0.0687)=0.0669 \mathrm{Mb} \cdot \mathrm{cc} / \mathrm{cc} \\
& \mathrm{E}_{\mathrm{d}}(\mathrm{v}=4.1) \sim(0.9863)^{1.5}(0.0797)=0.0778 \mathrm{Mb} \cdot \mathrm{cc} / \mathrm{cc} \\
& \mathrm{E}_{\mathrm{d}}(\mathrm{v}=6.5) \sim(0.9863)^{1.5}(0.0837)=0.0818 \mathrm{Mb} \cdot \mathrm{cc} / \mathrm{cc}
\end{aligned}
$$

Staying the same is $R_{1}, R_{2}, \omega$ and $\Gamma_{j}+1$. A adjusts automatically in the spreadsheet. We then vary $B$ to obtain the new JWL, which we compare here with the old:

\begin{tabular}{ccc} 
& LX-14 & LX-14 \\
& JWL & new density \\
\cline { 2 - 3 }$\rho_{0}(\mathrm{~g} / \mathrm{cc})$ & 1.825 & 1.80 \\
$\mathrm{~A}(\mathrm{Mb})$ & 7.74304 & 7.49773 \\
$\mathrm{~B}(\mathrm{Mb})$ & 0.20927 & 0.20264 \\
$\mathrm{C}(\mathrm{Mb})$ & 0.01390 & 0.01397 \\
$\mathrm{R}_{1}$ & 4.5 & 4.5 \\
$\mathrm{R}_{2}$ & 1.5 & 1.5 \\
$\omega$ & 0.33 & 0.33 \\
$\mathrm{E}_{\mathrm{o}}(\mathrm{Mb})$ & 0.1064 & 0.1047 \\
$\Gamma_{\mathrm{j}}+1$ & 3.80 & 3.80
\end{tabular}




$\begin{array}{ccc}P_{j} & 0.371 & 0.360 \\ D & 0.8783 & 0.8703 \\ \mathrm{v}_{j} & 0.737 & 0.737\end{array}$

In this process, $P_{j}$ changes as the 2.2 power and $A$ as the 2.3 .

\section{Problem: Calculating an HMX Derivitive}

Often, the unknown explosive has some cylinder data and is similiar to a well-known explosive. Here we consider an HMX explosive close enough in properties to LX-14. PBXW-9 is $92 \%$ HMX with 6\% di(2-ethylhexyl)adiapate and $2 \%$ Hycar 4054 with a density of $1.73 \mathrm{~g} / \mathrm{cc}$. The measured detonation velocity was $0.849 \mathrm{~cm} / \mu \mathrm{s}$. The data was taken with a full-wall LLNL-type cylinder at the $R-R_{0}$ values of 6 and $19 \mathrm{~mm} .{ }^{6}$ These points are averaged to estimate the value at 12 $\mathrm{mm}$. We have:

\begin{tabular}{cccccc}
$\mathrm{R}-\mathrm{R}_{\mathrm{o}}$ & & \multicolumn{2}{c}{$\mathrm{V}$ elocity $(\mathrm{mm} / \mathrm{\mu s})$} & \multicolumn{2}{c}{$\mathrm{E}_{\mathrm{d}}(\mathrm{Mb} \cdot \mathrm{cc} / \mathrm{cc})$} \\
\cline { 4 - 7 }$(\mathrm{mm})$ & $\mathrm{v}$ & PETN & PBXW-9 & PETN & PBXW-9 \\
\hline 6 & 2.2 & 1.575 & 1.55 & 0.0665 & 0.0644 \\
12.5 & 4.1 & 1.720 & 1.68 & 0.0781 & 0.0745 \\
19 & 6.5 & 1.775 & 1.74 & 0.0829 & 0.0797
\end{tabular}

The detonation energies are always referenced to PETN for simplicity. For example, we got the $\mathrm{v}=$ 2.2 detonation energy by calculating

$$
\mathrm{E}_{\mathrm{d}}(\mathrm{PBXW}-9,2.2)=(1.55 / 1.575)^{2}(0.665)=0.0644 \mathrm{Mbar} \cdot \mathrm{cc} / \mathrm{cc}
$$

The scalings are done directly against the LX-14 cylinders as before:

$$
\begin{aligned}
& \mathrm{D} \sim\left[(1.73 / 1.825)^{0.667}\right] 0.8783 \sim 0.848 \mathrm{~cm} / \mu \mathrm{s}, \text { compare with } 0.849 \text { measured } \\
& \mathrm{E}_{0} \sim(1.73 / 1.825)^{1.2}(0.1064) \sim 0.1015 \mathrm{Mbar} \cdot \mathrm{cc}
\end{aligned}
$$

We get: 


\begin{tabular}{cc} 
& PBXW-9 \\
\cline { 2 - 2 }$\rho_{\mathbf{o}}(\mathrm{g} / \mathrm{cc})$ & 1.73 \\
$\mathrm{~A}(\mathrm{Mb})$ & 6.79607 \\
$\mathrm{~B}(\mathrm{Mb})$ & 0.19417 \\
$\mathrm{C}(\mathrm{Mb})$ & 0.01417 \\
$\mathrm{R}_{1}$ & 4.5 \\
$\mathrm{R}_{2}$ & 1.5 \\
$\omega$ & 0.33 \\
$\mathrm{E}_{\mathbf{0}}(\mathrm{Mb} . \mathrm{cc} / \mathrm{cc})$ & 0.1015 \\
$\Gamma_{\mathrm{j}}+1$ & 3.80 \\
$\mathrm{P}_{\mathbf{j}}$ & 0.330 \\
$\mathrm{D}$ & 0.849 \\
$\mathrm{v}_{\mathbf{j}}$ & 0.737
\end{tabular}

\section{References}

1. P. C. Souers and L. C. Haselman, Jr., Detonation Equation of State at LLNL, 1993, LLNL Report UCRL-ID-116113 (March, 1994), pp. 3-1 to 3-7; 4-1 to 4-4, 5-1 to 5-20.

2. D. J. Steinberg, Equation of State and Strength Properties of Selected Materials, LLNL Report UCRL-MA-106439 (991).

3. P. A. Urtiew and B. Hayes, Parametric Study of the Dynamic JWL-EOS for Detonation Products, LLNL Report UCRL-JC-103218 (1990).

4. P. E. Kramer, HNS Cylinder Tests, Mason \& Hanger Pantex Plant Report MHSMP-75-17, Amarillo, TX (1975).

5. S. A. Aubert, U. S. Army Research, Development and Engineering Center, Picatinny Arsenal, NJ, private communication, 1994.

6. K. Alexander, Naval Surface Warfare Center Report NSWC TR 86-334, Silver Spring, MD, section sent as a Private Communication, 1994. 


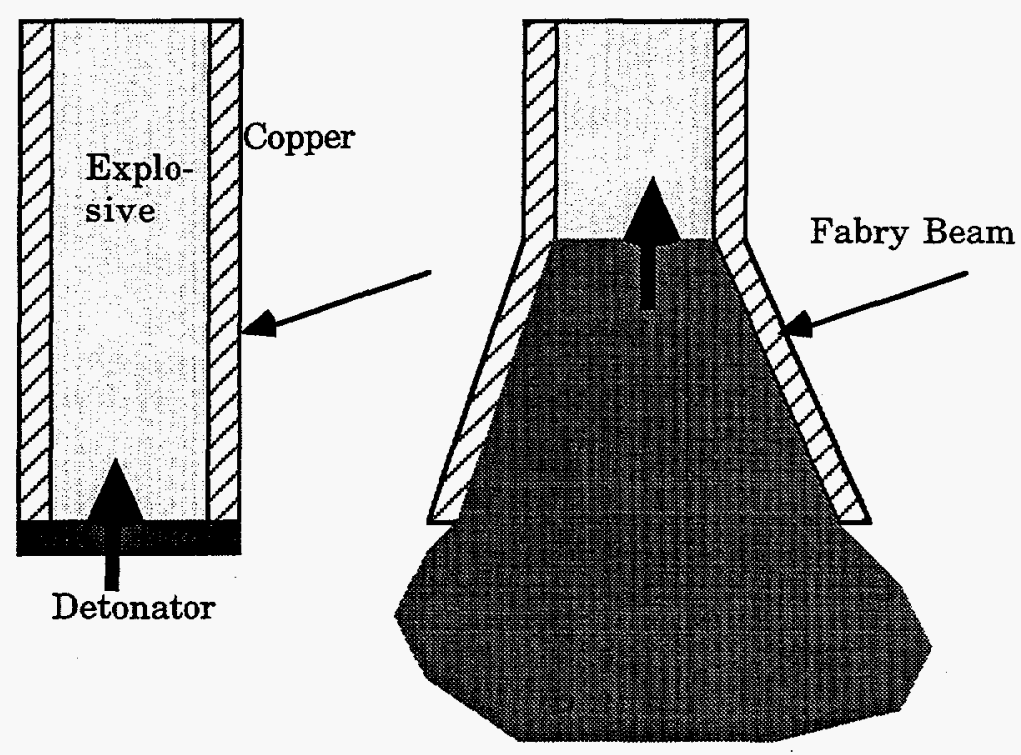

Fig. 3-1. Schematic of the Cylinder Test. The detonation front moves left to right. The velocity of the fanning copper walls is measured. Cylinder diameters include 1, 2, 4 and 8 -inches. Detonation is upward. 
Get $D$ (meas) and $E_{d}(v)$ from experiment. Get $R_{1} \cdot R_{2}$, and $E_{0}(\infty)$ from CHEQ or CHEETAH. Estimate $A, B$, and $\Gamma_{j}+1$.

$$
\begin{aligned}
& v_{j}=\Gamma_{j} /\left(\Gamma_{j}+1\right)=1-1 / \text { he } \\
& P_{j}=\frac{A\left(1-\omega / R_{1} v_{j}\right) \exp \left(-R_{1} v_{j}\right)+B\left(1-\omega / R_{2} v_{j}\right) \exp \left(-R_{2} v_{j}\right)+\omega E_{o}(\infty) / v_{j}}{1-\omega\left(1-v_{j}\right) / 2 v_{j}} \\
& C=v_{j}^{1+\omega}\left[P_{j}-A \exp \left(-R_{1} v_{j}\right)-B \exp \left(-R_{2} v_{j}\right)\right] \\
& E_{d}(v)=E_{o}-\left[\frac{A}{R_{1}} \exp \left(-R_{1} v\right)+\frac{B}{R_{2}} \exp \left(-R_{2} v\right)+\frac{C}{\omega v^{w}}\right] \quad \text { Do for } v=2.2,4.1 \text { and } 6.5 ;
\end{aligned}
$$

compare with experimental $\mathrm{E}_{\mathrm{d}}(\mathrm{v})$

$$
\begin{aligned}
& D=\left(\frac{1}{\rho_{0}} \frac{\partial P}{\partial v}\right)_{j}^{1 / 2}=\left\{\frac{1}{\rho_{0}}\left[A R_{1} \exp \left(-R_{1} v_{j}\right)+B R_{2} \exp \left(-R_{2} v_{j}\right)+\frac{(1+\omega) C}{v_{j}^{2+\omega}}\right]\right\}^{1 / 2} \text { compare with } D(\text { meas }) \\
& P_{j}=\rho_{0} D(\text { meas })^{2}\left(1-v_{j}\right) \\
& P_{j}=A \exp \left(-R_{1} v_{j}\right)+B \exp \left(-R_{2} v_{j}\right)+\frac{C}{v_{j}^{1+\omega}} \quad \text { compare these two } P_{j} \text { results }
\end{aligned}
$$

Reset $A, B, \Gamma_{j}+1$; reiterate to $0.01 \%$ agreement for $D$ and $P_{j} ; \pm 5 \%$ agreement for $E_{d}$.

Fig. 3-2. Path of cylinder JWL calculations starting with A and B and working to the energies. This is backwards to the old way of accepting energies as perfect and doing a linear omputation of A, B and $C . P_{j}$ and $E_{d}$ must be reiterated. 

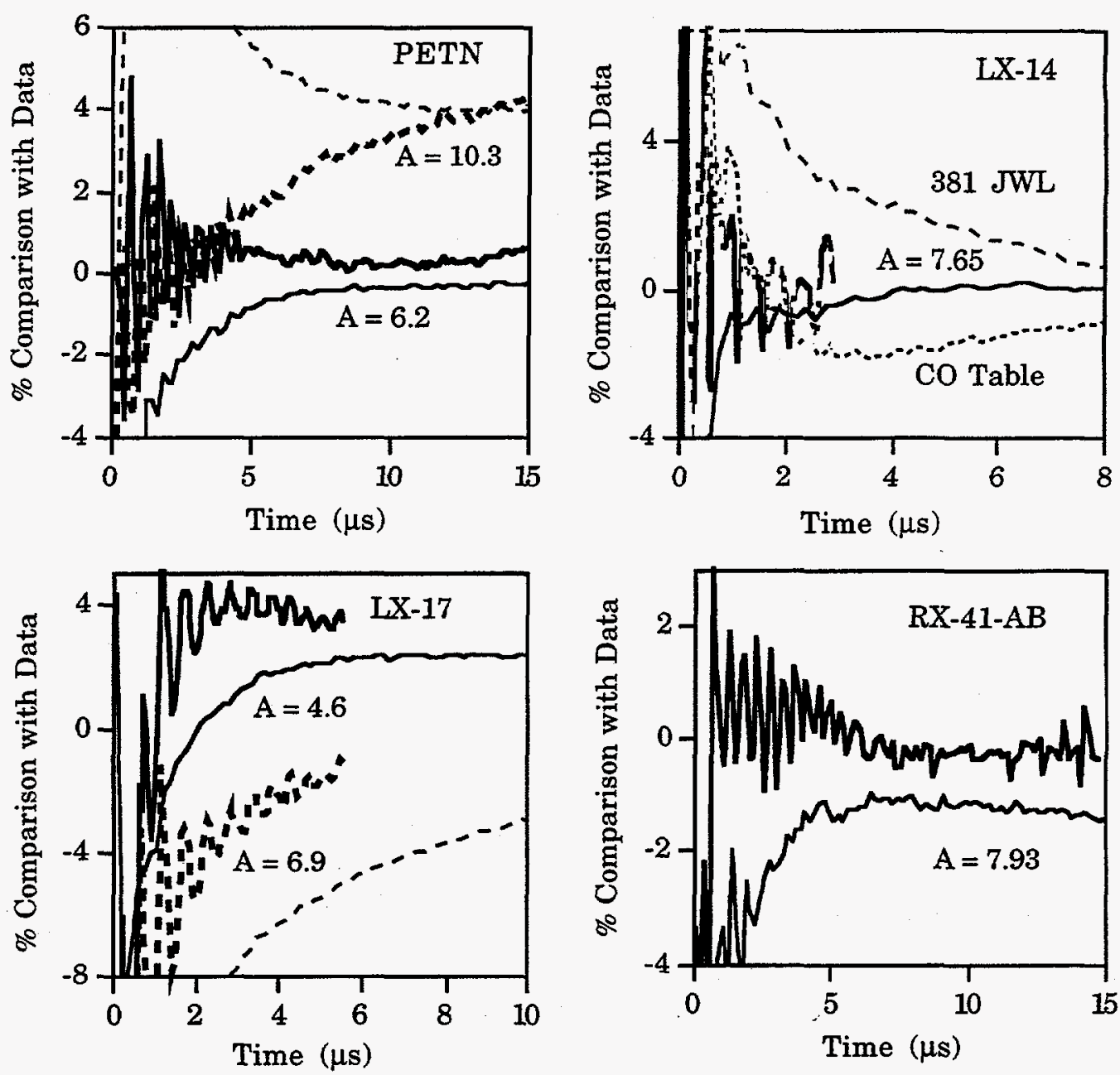

Fig. 3-3. \% comparison of code runs with experiment. a) PETN. The new standard is $A=6.2$ Fabry (heavy full line) and streak camera $R-R_{0}$ (light full). The old standard is $A=10.3$ with Fabry (heavy dashed) and $R-R_{0}$ (light dashed). b) $L X-14$. The new cylinder JWL $A=7.65$ is Fabry (heavy full) and $\mathrm{R}-\mathrm{R}_{0}$ (light full); also, we have the $381 \mathrm{R}-\mathrm{R}_{0}$ (dashed) and the CO Table (dotted). Their Fabrys are the same. c) $L X-17$. The best JWL is the $A=4.603$ with Fabry (heavy full) and $R-R_{0}$ (light full); not so good is the new $R_{1}=4.5 \mathrm{JWL}$ with $A=6.9$ Fabry (heavy dashed) and $R-R_{0}$ (dashed). d) $R X-41-A B$. Shown is the $A=7.93$ Fabry (heavy full) and $R-R_{0}$ (light full). 


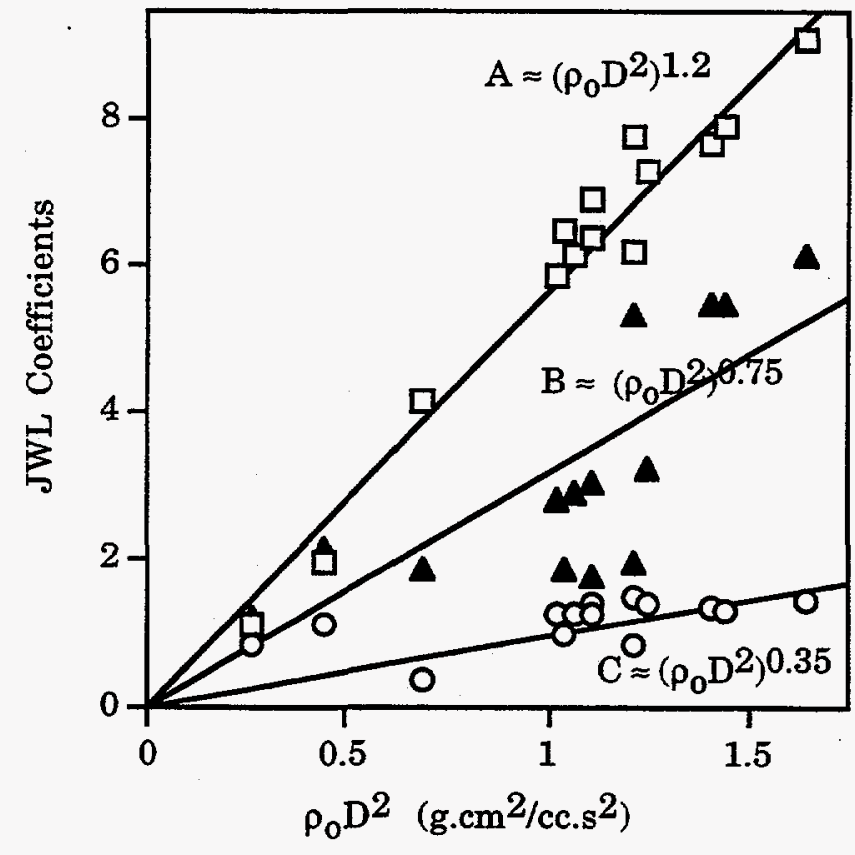

Fig. 3-4. JWL coefficients from Table 3-1 plotted as a function of $\rho_{0} D^{2}$. Only the coefficient $A$ is likely to be truly proportional. Units of $A$ are used on the $\mathrm{Y}$-axis. $\mathrm{B}$ is multiplied by 25 and $\mathrm{C}$ by 100. 
Table 3-1. List of JWL's run in 2-D cylinder calculations. The bold-face indicates the best fit. The $R_{1}=4.5 ; R_{2}=1.5$ JWL's give the best fit except for LX-17. Various famous old JWL's are included. Mbar units are used. "Fabry" is the \% agreement of the code with the measured jump-off velocity' "R - $R_{0}$ " is the \% agreement with the long-time streak camera distance. The $R_{1}=4.5$ JWL's are designed to be used in large volume far-field blast problems.

\begin{tabular}{|c|c|c|c|c|c|c|c|c|c|}
\hline & PETN & PETN & LX-14 & LX-14 & LX-14 & LX-17 & LX-17 & AFX-902 & HNS \\
\hline & Old & $\mathrm{A}=$ & 381 & 358 & $A=$ & LH & $A=$ & $A=$ & $A=$ \\
\hline & Std. & 6.22 & JWL & JWL & 7.65 & JWL & 6.92 & 7.76 & 1.12 \\
\hline \multirow{2}{*}{$\begin{array}{c}\rho_{0} \\
\mathrm{~A}\end{array}$} & 1.763 & 1.766 & 1.835 & 1.8358 & 1.825 & 1.900 & 1.909 & 1.742 & 1.001 \\
\hline & 10.32158 & 6.22045 & 7.55980 & 31.04400 & 7.65434 & 4.60300 & 6.92131 & 7.76067 & 1.12502 \\
\hline $\mathrm{B}$ & 0.90570 & 0.21465 & 0.22670 & 1.74430 & 0.21932 & 0.09544 & 0.07150 & 0.07895 & 0.04767 \\
\hline $\mathrm{C}$ & 0.037274 & 0.014966 & 0.009825 & 0.013035 & 0.013300 & 0.012650 & 0.012710 & 0.008192 & 0.008294 \\
\hline$R_{1}$ & 6.0 & 4.5 & 4.44 & 7.66 & 4.5 & 4.0 & 4.5 & 4.5 & 4.5 \\
\hline $\mathrm{R}_{2}$ & 2.6 & 1.5 & 1.5 & 2.65 & 1.5 & 1.7 & 1.5 & 1.5 & 1.5 \\
\hline$\omega$ & 0.57 & 0.29 & 0.30 & 0.40 & 0.33 & 0.48 & 0.26 & 0.30 & 0.31 \\
\hline$E_{0}$ & 0.1080 & 0.1120 & 0.101 & 0.095 & 0.1060 & 0.069 & 0.0860 & 0.0680 & 0.0400 \\
\hline \multirow{2}{*}{$\Gamma_{\mathrm{j}}+1$} & 3.83 & 3.66 & 3.755 & 3.998 & 3.77 & 3.654 & 4.06 & 4.13 & 3.40 \\
\hline & 0.8274 & 0.8283 & 0.883 & 0.883 & 0.8783 & 0.7596 & 0.7636 & 0.8340 & 0.5100 \\
\hline \multirow{2}{*}{$\begin{array}{l}P_{j} \\
v_{i}\end{array}$} & 0.315 & 0.331 & 0.381 & 0.358 & 0.373 & 0.300 & 0.274 & 0.293 & 0.077 \\
\hline & 0.739 & 0.727 & 0.734 & 0.750 & 0.735 & 0.726 & 0.754 & 0.758 & 0.706 \\
\hline$\frac{1}{\text { Fabry }}$ & -2 & 5 & 14 & 10 & -11 & -6 & -15 & none & none \\
\hline \multirow[t]{4}{*}{$R-R_{0}$} & 4 & 0 & -1 & -3 & 0 & 2 & 1 & -1 & 0 \\
\hline & NM & $\mathrm{RX}-26-\mathrm{AF}$ & $\mathrm{RX}-39-\mathrm{AB}$ & RX-41-AB & RX-45-AA & RX-47-AA & RX-48-AA & $\mathrm{RX}-52-\mathrm{AE}$ & TNM \\
\hline & $\mathrm{A}=$ & $\mathrm{A}=$ & $\mathrm{A}=$ & $\mathrm{A}=$ & $A=$ & $\mathrm{A}=$ & $\mathrm{A}=$ & $\mathrm{A}=$ & $A=$ \\
\hline & 1.98 & 7.31 & 9.08 & 7.92 & 6.46 & 6.13 & 6.37 & 5.84 & 4.14 \\
\hline \multirow{2}{*}{$\begin{array}{c}\rho_{0} \\
\mathrm{~A}\end{array}$} & 1.13 & 1.844 & 1.942 & 1.857 & 1.75 & 1.82 & 1.848 & 1.78 & 1.65 \\
\hline & 1.98182 & 7.31061 & 9.08741 & 7.92731 & 6.46425 & 6.13610 & 6.37869 & 5.84476 & 4.14336 \\
\hline \multirow{2}{*}{$\begin{array}{l}\mathrm{B} \\
\mathrm{C}\end{array}$} & 0.08723 & 0.13066 & 0.24627 & 0.21868 & 0.07543 & 0.11710 & 0.12196 & 0.11262 & 0.07499 \\
\hline & 0.011364 & 0.014105 & 0.014506 & 0.012978 & 0.009915 & 0.012730 & 0.014176 & 0.012821 & 0.003697 \\
\hline$R_{1}$ & 4.5 & 4.5 & 4.5 & 4.5 & 4.5 & 4.5 & 4.5 & 4.5 & 4.5 \\
\hline \multirow{2}{*}{$\begin{array}{c}\mathrm{R}_{2} \\
\omega\end{array}$} & 1.5 & 1.5 & 1.5 & 1.5 & 1.5 & 1.5 & 1.5 & 1.5 & 1.5 \\
\hline & 0.34 & 0.30 & 0.34 & 0.32 & 0.30 & 0.28 & 0.28 & 0.29 & 0.37 \\
\hline \multirow{3}{*}{$\begin{array}{c}E_{0} \\
\Gamma_{j}+1 \\
D\end{array}$} & 0.0569 & 0.0960 & 0.1180 & 0.1070 & 0.0690 & 0.0880 & 0.0950 & 0.0850 & 0.0374 \\
\hline & 3.44 & 3.92 & 3.80 & 3.79 & 4.06 & 3.89 & 3.88 & 3.88 & 3.99 \\
\hline & 0.6280 & 0.8240 & 0.9208 & 0.8814 & 0.7710 & 0.7650 & 0.7760 & 0.7570 & 0.6450 \\
\hline \multirow{2}{*}{$P_{j}$} & 0.130 & 0.319 & 0.433 & 0.381 & 0.256 & 0.274 & 0.287 & 0.263 & 0.172 \\
\hline & 0.709 & 0.745 & 0.737 & 0.736 & 0.754 & 0.743 & 0.742 & 0.742 & 0.749 \\
\hline Fabry & none & none & 16 & 10 & -15 & 0 & 2 & -7 & none \\
\hline R-R & 3 & -1 & -1 & -1 & 0 & -1 & -2 & -1 & 3 \\
\hline
\end{tabular}


Table 3-2. The CO Look-Up Table for LX-14. The table contains $\eta(1 / v)$ and $f$. The volume $v$ is listed for comparison.

\begin{tabular}{ccc|ccc}
$\rho_{\mathrm{o}}$ & 1.835 & \multicolumn{1}{c}{$\eta$} & $\mathrm{f}$ & $\mathrm{v}$ \\
$\mathrm{D}$ & 0.8815 & & 1.05 & 1.7030 & 0.9524 \\
$\mathrm{E}_{\mathrm{o}}$ & 0.100 & & 1.00 & 1.6576 & 1.0000 \\
$\Gamma_{\mathrm{j}}+1$ & 3.660 & & 0.95 & 1.6034 & 1.0526 \\
$\mathrm{P}_{\mathrm{j}}$ & 0.340 & & 0.90 & 1.5440 & 1.1111 \\
& & & 0.85 & 1.4807 & 1.1765 \\
$\eta$ & $\mathrm{f}$ & $\mathrm{v}$ & 0.80 & 1.4127 & 1.2500 \\
\hline 2.00 & 2.2147 & 0.5000 & 0.75 & 1.3414 & 1.3333 \\
1.90 & 2.1795 & 0.5263 & 0.70 & 1.2700 & 1.4286 \\
1.80 & 2.1380 & 0.5556 & 0.65 & 1.2012 & 1.5385 \\
1.70 & 2.0891 & 0.5882 & 0.60 & 1.1355 & 1.6667 \\
1.60 & 2.0313 & 0.6250 & 0.55 & 1.0722 & 1.8182 \\
1.50 & 1.9650 & 0.6667 & 0.50 & 1.0126 & 2.0000 \\
1.45 & 1.9285 & 0.6897 & 0.45 & 0.9560 & 2.2222 \\
1.40 & 1.8931 & 0.7143 & 0.40 & 0.9040 & 2.5000 \\
1.35 & 1.8606 & 0.7407 & 0.35 & 0.8563 & 2.8571 \\
1.3131 & 1.8424 & $\mathbf{0 . 7 6 1 6}$ & 0.30 & 0.8132 & 3.3333 \\
1.30 & 1.8360 & 0.7692 & 0.25 & 0.7771 & 4.0000 \\
1.25 & 1.8104 & 0.8000 & 0.20 & 0.7528 & 5.0000 \\
1.20 & 1.7890 & 0.8333 & 0.15 & 0.7487 & 6.6667 \\
1.15 & 1.7678 & 0.8696 & 0.10 & 0.7857 & 10.000 \\
1.10 & 1.7390 & 0.9091 & 0.05 & 0.9288 & 20.000
\end{tabular}


Table 3-3. Complete list of cylinder data. $\mathrm{E}_{\mathrm{d}}$ is the energy of detonation. "Ptx" is Pantex and "A" is ARDEC. The PETN standard is in bold-face.

\begin{tabular}{|c|c|c|c|c|c|c|c|c|c|c|c|}
\hline & \multirow{2}{*}{$\begin{array}{l}\text { Explo- } \\
\text { sive }\end{array}$} & \multirow{2}{*}{$\begin{array}{l}\text { Den- } \\
\text { sity } \\
\text { (g/cc) }\end{array}$} & \multirow{2}{*}{$\begin{array}{l}\text { Shot } \\
\text { No. }\end{array}$} & \multirow{2}{*}{$\begin{array}{c}\text { Det } \\
\text { Vel } \\
(\mathrm{mm} / \\
\mu \mathrm{s})\end{array}$} & \multirow{2}{*}{$\begin{array}{l}\text { Dia } \\
\text { (in) }\end{array}$} & \multicolumn{3}{|c|}{$\begin{array}{c}\text { Scaled Wall } \\
\text { Velocity }(\mathrm{mm} / \mu \mathrm{s})\end{array}$} & \multicolumn{3}{|c|}{ Approx $E_{d}(M b . c c)$} \\
\hline & & & & & & 2.2 & 4.1 & 6.5 & 2.2 & 4.1 & 6.5 \\
\hline HALF & PETN & 1.766 & 586 & 8.28 & 1 & 2.040 & 2.217 & 2.292 & 0.0658 & 0.0775 & 0.0823 \\
\hline WALL & LX-04-1 & 1.869 & 547 & 8.49 & 2 & 2.002 & 2.158 & 2.231 & 0.0634 & 0.0734 & 0.0780 \\
\hline FABRY, & RX-39-AB & 1.942 & 564 & 9.21 & 1 & 2.224 & 2.396 & 2.462 & 0.0782 & 0.0905 & 0.0950 \\
\hline \multirow[t]{2}{*}{$\mathrm{Cu}$} & $\mathrm{RX}-41-\mathrm{AB}$ & 1.857 & 560 & 8.81 & 1 & 2.086 & 2.262 & 2.327 & 0.0688 & 0.0807 & 0.0849 \\
\hline & $\mathrm{RX}-52-\mathrm{AE}$ & 1.780 & 585 & 7.57 & 2 & 1.728 & 1.874 & 1.938 & 0.0472 & 0.0554 & 0.0589 \\
\hline HALF & PETN & 1.766 & 586 & 8.28 & $\mathbf{1}$ & 2.080 & 2.283 & 2.376 & & & \\
\hline WALL & PETN & 1.761 & 511 & 8.32 & 1 & 2.086 & 2.286 & 2.377 & & & \\
\hline STREAK & Average & 1.764 & & 8.30 & & 2.083 & 2.285 & 2.377 & 0.0658 & 0.0775 & 0.0823 \\
\hline \multirow[t]{8}{*}{$\mathrm{Cu}$} & COMP B & 1.714 & 583 & 7.90 & 2 & 2.109 & 2.248 & 2.321 & 0.0675 & 0.0750 & 0.0785 \\
\hline & LX-04-1 & 1.869 & 547 & 8.49 & 2 & 2.062 & 2.245 & 2.310 & 0.0645 & 0.0748 & 0.0778 \\
\hline & PETN & 1.261 & 264 & 6.76 & 1 & 1.576 & 1.780 & 1.857 & 0.0377 & 0.0470 & 0.0503 \\
\hline & PETN & 1.507 & 265 & 7.49 & 1 & 1.842 & 2.048 & 2.118 & 0.0515 & 0.0623 & 0.0654 \\
\hline & $R X-39-A B$ & 1.942 & 564 & 9.21 & 1 & 2.300 & 2.474 & 2.545 & 0.0802 & 0.0909 & 0.0944 \\
\hline & $\mathrm{RX}-41-\mathrm{AB}$ & 1.857 & 560 & 8.81 & 1 & 2.135 & 2.327 & 2.411 & 0.0691 & 0.0804 & 0.0847 \\
\hline & $\mathrm{RX}-52-\mathrm{AE}$ & 1.780 & 585 & 7.57 & 2 & 1.774 & 1.924 & 1.998 & 0.0477 & 0.0550 & 0.0582 \\
\hline & \multirow[t]{4}{*}{$\overline{\mathrm{LX}}-14$} & 1.819 & 469 & 8.77 & 1 & 2.120 & 2.323 & 2.379 & \multirow[b]{5}{*}{0.0695} & \multirow[b]{5}{*}{0.0802} & \multirow[b]{5}{*}{0.0840} \\
\hline & & 1.821 & 510 & 8.78 & 2 & 2.153 & 2.318 & 2.408 & & & \\
\hline & & 1.83 & 520 & 8.79 & 2 & 2.128 & 2.320 & 2.397 & & & \\
\hline & & 1.83 & 521 & 8.80 & 2 & 2.163 & 2.332 & 2.418 & & & \\
\hline & Average & 1.825 & & 8.78 & & 2.141 & 2.323 & 2.401 & & & \\
\hline & \multirow[t]{5}{*}{$\mathrm{LX}-17$} & 1.912 & 439 & 7.66 & 2 & 1.740 & 1.890 & 1.950 & \multirow[b]{6}{*}{0.0460} & \multirow[b]{6}{*}{0.0522} & \multirow[b]{6}{*}{0.0549} \\
\hline & & 1.904 & 470 & 7.62 & 2 & 1.750 & 1.856 & 1.933 & & & \\
\hline & & 1.906 & 471 & 7.62 & 2 & 1.724 & 1.861 & 1.930 & & & \\
\hline & & 1.908 & 522 & 7.62 & 2 & 1.741 & 1.880 & & & & \\
\hline & & 1.917 & 523 & 7.66 & 2 & 1.754 & 1.884 & 1.953 & & & \\
\hline & Average & 1.909 & & 7.64 & & 1.742 & 1.874 & 1.942 & & & \\
\hline & \multirow[t]{2}{*}{$\overline{\text { PBX-9404 }}$} & 1.849 & 512 & 8.78 & 2 & 2.180 & 2.350 & 2.430 & \multirow{3}{*}{0.0714} & \multirow[b]{3}{*}{0.0813} & \multirow[b]{3}{*}{0.0855} \\
\hline & & 1.835 & 528 & 8.77 & 2 & 2.160 & 2.330 & 2.415 & & & \\
\hline & Average & 1.842 & & 8.77 & & 2.170 & 2.340 & 2.423 & & & \\
\hline & \multirow[t]{6}{*}{$\mathrm{RX}-26-\mathrm{AF}$} & 1.844 & 460 & 8.24 & 2 & 1.955 & 2.120 & 2.160 & & & \\
\hline & & 1.845 & 495 & 8.25 & 2 & 1.945 & 2.100 & 2.165 & & & \\
\hline & & 1.845 & 484 & 8.24 & 2 & 1.945 & 2.095 & 2.190 & & & \\
\hline & & 1.846 & 455 & 8.24 & 1 & 1.940 & 2.085 & 2.155 & & & \\
\hline & & 1.844 & 490 & 8.24 & 2 & 1.940 & 2.095 & 2.160 & & & \\
\hline & & 1.840 & 444 & 8.23 & 2 & 1.940 & 2.095 & 2.170 & & & \\
\hline & Average & 1.842 & & 8.23 & & 1.940 & 2.095 & 2.165 & 0.0571 & 0.0652 & 0.0683 \\
\hline $\begin{array}{c}\text { F. WALL } \\
\text { FABRY }\end{array}$ & Octol & 1.809 & 593 & 8.23 & 1 & 1.510 & 1.647 & 1.708 & 0.0631 & 0.0755 & 0.0820 \\
\hline FULL- & $\overline{\text { PETN }}$ & 1.765 & 187 & & 1 & 1.570 & 1.710 & 1.780 & & & \\
\hline WALL & PETN & 1.765 & 209 & 8.28 & 1 & 1.580 & 1.730 & 1.770 & & & \\
\hline STREAK & Average & 1.765 & & 8.28 & & 1.575 & 1.720 & 1.775 & 0.0658 & 0.0775 & 0.0823 \\
\hline
\end{tabular}




\begin{tabular}{|c|c|c|c|c|c|c|c|c|c|c|c|}
\hline \multirow[t]{2}{*}{$\begin{array}{c}\text { Table 3-3 } \\
\text { part } 2\end{array}$} & \multirow{2}{*}{$\begin{array}{l}\text { Explo- } \\
\text { sive }\end{array}$} & \multirow{2}{*}{$\begin{array}{l}\text { Den- } \\
\text { sity } \\
(\mathrm{g} / \mathrm{cc})\end{array}$} & \multirow{2}{*}{$\begin{array}{l}\text { Shot } \\
\text { No. }\end{array}$} & \multirow{2}{*}{$\begin{array}{c}D \\
(\mathrm{~mm} / \\
\mu \mathrm{s})\end{array}$} & \multirow{2}{*}{$\begin{array}{l}\text { Dia } \\
\text { (in) }\end{array}$} & \multicolumn{3}{|c|}{$\begin{array}{c}\text { Scaled Wall } \\
\text { Velocity (mm/ } / \mathrm{s})\end{array}$} & \multicolumn{3}{|c|}{ Approx $E_{d}(k J / c c)$} \\
\hline & & & & & & 2.2 & 4.1 & 6.5 & 2.2 & 4.1 & 6.5 \\
\hline FULLr & $\overline{B T F}$ & 1.852 & 270 & 8.49 & 1 & 1.605 & 1.755 & 1.835 & 0.0683 & 0.0807 & 0.0880 \\
\hline WALL & 1,2-DP & 1.256 & 115 & 5.97 & 1 & 1.090 & 1.235 & 1.310 & 0.0315 & 0.0400 & 0.0448 \\
\hline STREAK & FEFO & 1.607 & 513 & 7.45 & 1 & 1.340 & 1.490 & 1.560 & 0.0476 & 0.0582 & 0.0636 \\
\hline \multirow[t]{39}{*}{$\mathrm{Cu}$} & FM-1 & 1.509 & 514 & 6.57 & 1 & 1.258 & 1.390 & 1.455 & 0.0420 & 0.0506 & 0.0553 \\
\hline & HMX & 1.894 & 106,7 & 9.10 & 1 & 1.650 & 1.820 & 1.883 & 0.0722 & 0.0868 & 0.0926 \\
\hline & HMX & 1.188 & 198,9 & 6.68 & 1 & 1.173 & 1.314 & 1.384 & 0.0365 & 0.0452 & 0.0500 \\
\hline & HNB & 1.965 & 326 & 9.34 & 0.75 & 1.700 & 1.880 & 1.955 & 0.0767 & 0.0926 & 0.0998 \\
\hline & HNS & 1.681 & Ptx & 7.08 & 1 & 1.255 & 1.385 & 1.458 & 0.0418 & 0.0503 & 0.0555 \\
\hline & HNS & 1.655 & Ptx & 7.03 & 1 & 1.228 & 1.362 & 1.433 & 0.0400 & 0.0486 & 0.0536 \\
\hline & HNS & 1.610 & Ptx & 6.96 & 1 & 1.210 & 1.335 & 1.393 & 0.0388 & 0.0467 & 0.0507 \\
\hline & HNS & 1.597 & Ptx & 6.96 & 1 & 1.229 & 1.340 & 1.413 & 0.0401 & 0.0470 & 0.0522 \\
\hline & HNS & 1.504 & Ptx & 6.70 & 1 & 1.170 & 1.297 & 1.368 & 0.0363 & 0.0441 & 0.0489 \\
\hline & HNS & 1.402 & Ptx & 6.34 & 1 & 1.081 & 1.207 & 1.266 & 0.0310 & 0.0382 & 0.0419 \\
\hline & HNS & 1.200 & Ptx & 5.74 & 1 & 0.959 & 1.073 & 1.145 & 0.0244 & 0.0302 & 0.0342 \\
\hline & HNS & 1.001 & Ptx & 5.10 & 1 & 0.817 & 0.931 & 0.981 & 0.0177 & 0.0227 & 0.0251 \\
\hline & NM & 1.13 & 391 & 6.28 & 2 & 1.045 & 1.180 & 1.230 & 0.0290 & 0.0365 & 0.0395 \\
\hline & NNE & 1.034 & 324 & 5.31 & 2 & 0.836 & 0.935 & 0.990 & 0.0185 & 0.0229 & 0.0256 \\
\hline & PETN & 1.498 & 262 & 7.44 & 1 & 1.365 & 1.533 & 1.601 & 0.0494 & 0.0616 & 0.0670 \\
\hline & PETN & 1.266 & 261 & 6.66 & 1 & 1.162 & 1.305 & 1.381 & 0.0358 & 0.0446 & 0.0498 \\
\hline & $\mathrm{PF}$ & 1.833 & 194 & 7.29 & 1 & 1.395 & 1.525 & 1.585 & 0.0516 & 0.0609 & 0.0656 \\
\hline & RX-23-AA & 1.424 & 402 & 8.65 & 2 & 1.356 & 1.499 & 1.541 & 0.0488 & 0.0589 & 0.0620 \\
\hline & $\mathrm{RX}-23-\mathrm{AB}$ & 1.356 & 378 & 7.48 & 2 & 1.088 & 1.179 & & 0.0314 & 0.0364 & 0.0000 \\
\hline & $\mathrm{RX}-23-\mathrm{AB}$ & 1.356 & 246 & & 1 & 1.075 & 1.182 & 1.210 & 0.0307 & 0.0366 & 0.0382 \\
\hline & $\mathrm{RX}-23-\mathrm{AC}$ & 1.136 & 245 & 7.88 & 2 & 1.077 & 1.175 & 1.219 & 0.0308 & 0.0362 & 0.0388 \\
\hline & TATB & $1.80^{*}$ & 322 & 7.48 & 2 & 1.281 & 1.392 & 1.448 & 0.0435 & 0.0508 & 0.0548 \\
\hline & TATB & 1.854 & 379 & 7.68 & 2 & 1.312 & 1.417 & 1.470 & 0.0457 & 0.0526 & 0.0564 \\
\hline & TNAZ & 1.83 & $\mathrm{~A}$ & 8.75 & 1 & 1.64 & & 1.87 & 0.0713 & & 0.0913 \\
\hline & TNGU & 1.885 & 413 & 8.71 & 1 & 1.600 & 1.750 & 1.825 & 0.0679 & 0.0802 & 0.0870 \\
\hline & TNM & 1.650 & 474 & 6.45 & 1 & 1.000 & 1.095 & 1.130 & 0.0265 & 0.0314 & 0.0334 \\
\hline & TNT & 1.632 & $\begin{array}{c}140-1 \\
271\end{array}$ & 7.07 & 2 & 1.210 & 1.355 & 1.410 & 0.0388 & 0.0481 & 0.0519 \\
\hline & AFX-902 & 1.742 & 407 & 8.34 & 2 & 1.297 & 1.384 & 1.417 & 0.0446 & 0.0502 & 0.0524 \\
\hline & $\mathrm{C}-4$ & 1.601 & 289 & 8.19 & 1 & 1.378 & 1.522 & 1.582 & 0.0504 & 0.0607 & 0.0654 \\
\hline & LX-14 & 1.83 & 353 & 8.86 & 1 & 1.59 & 1.73 & 1.79 & 0.0671 & 0.0784 & 0.0837 \\
\hline & $\mathrm{RX}-27-\mathrm{AD}$ & 1.638 & 515 & 6.93 & 1 & 1.187 & 1.320 & 1.377 & 0.0374 & 0.0456 & 0.0495 \\
\hline & $\mathrm{RX}-36-\mathrm{AH}$ & 1.83 & 501 & 8.51 & 1 & 1.573 & 1.716 & 1.785 & 0.0656 & 0.0771 & 0.0832 \\
\hline & $\mathrm{RX}-43-\mathrm{AC}$ & 1.772 & 574 & 7.51 & 1 & 1.253 & 1.364 & 1.412 & 0.0416 & 0.0487 & 0.0521 \\
\hline & $\mathrm{RX}-45-\mathrm{AA}$ & 1.752 & 575 & 7.71 & 1 & 1.243 & 1.340 & 1.382 & 0.0410 & 0.0470 & 0.0499 \\
\hline & $\mathrm{RX}-47-\mathrm{AA}$ & 1.820 & 578 & 7.65 & 1 & 1.351 & 1.480 & 1.532 & 0.0484 & 0.0574 & 0.0613 \\
\hline & $\mathrm{RX}-48-\mathrm{AA}$ & 1.848 & 579 & 7.76 & 1 & 1.384 & 1.521 & 1.585 & 0.0508 & 0.0606 & 0.0656 \\
\hline & \multirow[t]{2}{*}{ LX-17 } & 1.900 & 349 & 7.63 & 2 & 1.293 & 1.409 & 1.453 & & & \\
\hline & & 1.875 & 434 & 7.55 & 2 & 1.29 & 1.41 & 1.46 & & & \\
\hline & Average & 1.874 & & 7.70 & & 1.294 & 1.408 & 1.454 & 0.0444 & 0.0519 & 0.0552 \\
\hline
\end{tabular}




\begin{tabular}{|c|c|c|c|c|c|c|c|c|c|c|c|}
\hline \multirow[t]{2}{*}{$\begin{array}{c}\text { Table 3-3 } \\
\text { part } 3\end{array}$} & \multirow{2}{*}{$\begin{array}{l}\text { Explo- } \\
\text { sive }\end{array}$} & \multirow{2}{*}{$\begin{array}{l}\text { Den- } \\
\text { sity } \\
(\mathrm{g} / \mathrm{cc})\end{array}$} & \multirow{2}{*}{$\begin{array}{l}\text { Shot } \\
\text { No. }\end{array}$} & \multirow{2}{*}{$\begin{array}{c}\mathrm{D} \\
(\mathrm{mm} / \\
\mu \mathrm{s})\end{array}$} & \multirow{2}{*}{$\begin{array}{l}\text { Dia } \\
\text { (in) }\end{array}$} & \multicolumn{3}{|c|}{$\begin{array}{c}\text { Scaled Wall } \\
\text { Velocity }(\mathrm{mm} / \mu \mathrm{s})\end{array}$} & \multicolumn{3}{|c|}{ Approx $\mathrm{E}_{\mathrm{d}}(\mathrm{kJ} / \mathrm{cc})$} \\
\hline & & & & & & 2.2 & 4.1 & 6.5 & 2.2 & 4.1 & 6.5 \\
\hline & PBX-9404 & 1.845 & 237 & 8.77 & 1 & 1.610 & 1.735 & 1.810 & & & \\
\hline & & 1.842 & 293 & 8.76 & 1 & 1.600 & 1.740 & 1.800 & & & \\
\hline & & 1.844 & 235 & 8.76 & 1 & 1.600 & 1.765 & 1.825 & & & \\
\hline & & 1.843 & 273 & 8.76 & 1 & 1.590 & 1.730 & 1.785 & & & \\
\hline & Average & 1.844 & & 8.76 & & 1.603 & 1.747 & 1.812 & 0.0682 & 0.0799 & 0.0857 \\
\hline HALF- & LX-17-1 & 1.91 & 554 & 7.65 & 2 & 1.394 & 1.498 & 1.540 & & & \\
\hline
\end{tabular}

Table 3-4. JWL' for explosives not run with the code. For PBX-9404, " $\mathrm{H}$ " is the more recent half-wall shot and "F" is the full-wall. These JWL's are designed to be used to large volumes in far-field blast problems. Mbar units are used.

\begin{tabular}{|c|c|c|c|c|c|c|c|c|c|}
\hline & BTF & C-4 & Comp-B & FEFO & HMX & $\mathrm{HMX}$ & HNB & HNS & $L X-04$ \\
\hline \multirow{2}{*}{$\begin{array}{c}\rho_{0} \\
\mathbf{A}\end{array}$} & 1.852 & 1.601 & 1.714 & 1.607 & 1.894 & 1.188 & 1.965 & 1.681 & 1.869 \\
\hline & 7.02570 & 5.97404 & 4.88963 & 4.64961 & 8.81453 & 2.22436 & 9.90453 & 4.63191 & 7.44450 \\
\hline $\mathrm{B}$ & 0.21901 & 0.13903 & 0.28264 & 0.12455 & 0.20902 & 0.12689 & 0.19758 & 0.10309 & 0.20923 \\
\hline C & 0.01587 & 0.01314 & 0.00799 & 0.01660 & 0.01565 & 0.01215 & 0.01807 & 0.01319 & 0.00996 \\
\hline$R_{1}$ & 4.5 & 4.5 & 4.5 & 4.5 & 4.5 & 4.5 & 4.5 & 4.5 & 4.5 \\
\hline $\mathbf{R}_{2}$ & 1.5 & 1.5 & 1.5 & 1.5 & 1.5 & 1.5 & 1.5 & 1.5 & 1.5 \\
\hline$\omega$ & 0.33 & 0.32 & 0.30 & 0.31 & 0.34 & 0.30 & 0.22 & 0.27 & 0.33 \\
\hline$E_{0}$ & 0.1120 & 0.0870 & 0.0940 & 0.0930 & 0.1140 & 0.0720 & 0.1520 & 0.0840 & 0.0930 \\
\hline \multirow{2}{*}{$\begin{array}{c}\Gamma_{\mathrm{j}}+1 \\
\mathrm{D}\end{array}$} & 3.71 & 3.82 & 3.45 & 3.70 & 3.84 & 3.34 & 3.89 & 3.795 & 3.80 \\
\hline & 0.8490 & 0.8190 & 0.7900 & 0.7450 & 0.9100 & 0.6680 & 0.9340 & 0.7080 & 0.8490 \\
\hline \multirow{3}{*}{$\begin{array}{l}P_{j} \\
v_{j}\end{array}$} & 0.360 & 0.281 & 0.310 & 0.241 & 0.408 & 0.159 & 0.441 & 0.222 & 0.355 \\
\hline & 0.730 & 0.738 & 0.710 & 0.730 & 0.740 & 0.701 & 0.743 & 0.736 & 0.737 \\
\hline & Octol & PBX-9404 & PBX-9404 & PETN & PETN & $\mathrm{RX}-27-\mathrm{AD}$ & TNAZ & TNGU & TNT \\
\hline \multirow{2}{*}{$\begin{array}{c}\rho_{0} \\
\mathrm{~A}\end{array}$} & 1.809 & $1.842 \mathrm{H}$ & $1.844 \mathrm{~F}$ & 1.263 & 1.503 & 1.638 & 1.83 & 1.885 & 1.632 \\
\hline & 6.42274 & 7.62770 & 7.73037 & 2.64744 & 3.99585 & 4.37298 & 7.39357 & 7.89524 & 4.54864 \\
\hline B & 0.20442 & 0.23276 & 0.21629 & 0.11501 & 0.17381 & 0.09548 & 0.24083 & 0.20668 & 0.10119 \\
\hline $\mathrm{C}$ & 0.014660 & 0.01257 & 0.01339 & 0.01256 & 0.01357 & 0.01119 & 0.01388 & 0.01429 & 0.01093 \\
\hline \multirow{3}{*}{$\begin{array}{c}R_{1} \\
R_{2} \\
\omega\end{array}$} & 4.5 & 4.5 & 4.5 & 4.5 & 4.5 & 4.5 & 4.5 & 4.5 & 4.5 \\
\hline & 1.5 & 1.5 & 1.5 & 1.5 & 1.5 & 1.5 & 1.5 & 1.5 & 1.5 \\
\hline & 0.32 & 0.33 & 0.33 & 0.25 & 0.27 & 0.30 & 0.30 & 0.30 & 0.25 \\
\hline \multirow{3}{*}{$\begin{array}{c}E_{0} \\
\Gamma_{j}+1 \\
D\end{array}$} & 0.105 & 0.1060 & 0.1060 & 0.0810 & 0.0960 & 0.0700 & 0.1150 & 0.1120 & 0.0780 \\
\hline & 3.70 & 3.75 & 3.78 & 3.47 & 3.52 & 3.82 & 3.71 & 3.80 & 3.82 \\
\hline & 0.8233 & 0.8770 & 0.8760 & 0.6760 & 0.7490 & 0.6930 & 0.8750 & 0.8710 & 0.7070 \\
\hline \multirow{2}{*}{$\begin{array}{l}P_{j} \\
v_{j}\end{array}$} & 0.331 & 0.378 & 0.374 & 0.166 & 0.240 & 0.206 & 0.378 & 0.376 & 0.214 \\
\hline & 0.730 & 0.733 & 0.735 & 0.712 & 0.716 & 0.738 & 0.730 & 0.737 & 0.738 \\
\hline
\end{tabular}





\section{Chapter 4. More Cylinders}

\section{Comparing Metal-Pushing}

The detonation energy/velocity-squared relation is quite powerful for comparing explosives fired with the same cylinder wall thicknesses as long as they are close to being ideal. Fig. 4-1 compares the velocity-squared ratio of TATB (cylinder \#379, $1.854 \mathrm{~g} / \mathrm{cc}$ ) with TNT (\#140-141, 1.634 g/cc), where both cylinders were 2-inch diameter, full-wall. The data was the distance measured by the streak camera, $R-R_{0}$ versus time. Both these were divided by 2 to scale to 1 -inch cylinders, and the derivitive was taken. The data was then replotted in terms of wall velocity versus $R-R_{0}$. The distance was converted into geometric volume, $v g$, by

$$
\mathrm{v}_{\mathrm{g}}=\left(1+\frac{\mathrm{R}-\mathrm{R}_{\mathrm{o}}}{\mathrm{S}_{\mathrm{o}}}\right)^{2}
$$

where $S_{0}$ is the initial inner radius. From the previous report, ${ }^{1}$ we equate the geometric cylinder volume to the approximate actual reaction gas volume, $\mathrm{va}_{\mathbf{a}}$, by

$$
\mathrm{v}=\left\{1.01\left[1-\exp \left(-1.8 \mathrm{v}_{\mathrm{g}}\right)\right]+0.003 \mathrm{v}_{\mathrm{g}}\right\} \mathrm{v}_{\mathrm{g}}
$$

When $v_{g}=1.00, v \approx 0.85$. As shown below, this is very close to the detonation volume at the edge of a cylinder.

We now are able to plot $[\mathrm{u}(\mathrm{TATB}) / \mathrm{u}(\mathrm{TNT})]^{2}$ as a function of the relative volume as shown in Fig. 4-1.

\section{Using CHEETAH to Generate JWL's (with Larry Fried)}

Larry Fried has written CHEETAH, the successor thermochemical code to TIGER. ${ }^{2-4}$ The model is BKW, the library is that Sandia's Hobbs and Baer ${ }^{5}$ and the normalization is BKWC (for CHEETAH). Besides being more robust, faster and having better summary print-outs than TIGER, CHEETAH's normalization has improved features. TIGER concentrated solely on the C-J point, while CHEETAH adds cylinder data at the three special volumes of 2.2, 4.1 and 6.5 and detonation 
energies at $1 \mathrm{~atm}$. A weighting of $50 \%$ is given to detonation velocity, $25 \%$ to C-J pressure and $25 \%$ to the adiabat. Agreement is set to $\pm 1 \%$ detonation velocity, $\pm 10 \%$ C-J pressure, $\pm 5 \%$ detonation energy on the adiabat, $\pm 5 \% 1 \mathrm{~atm}$ total energy of detonation from old cylinder JWL's and $\pm 3 \%$ total energy of detonation from calorimetry. A total of 34 CHNO explosives were incorporated into the normalization. Most were close enough to being ideal so that the cylinder data could be considered almost infinite-diameter. Two were highly non-ideal ANFO explosives for which actual infinitediameter detonation velocities were measured. The fit shifts the freeze temperature from the traditional $1800 \mathrm{~K}$ to an empirical best fit at $2145 \mathrm{~K}$.

CHEETAH adiabats are now locked to real data. This removes the rapidly plunging adiabats generally seen in TIGER. The adiabats still drop too fast in some cases (eg. nitroguanidine) so that the $1 \mathrm{~atm}$ temperature would be far below room temperature. The code now halts at the 1 atm point, wherever it is encountered.

The experimental CHEETAH V1.0 now contains a JWL fit modeled on the results already shown in this chapter. The total energy is considered to be the infinite-volume result, $\mathrm{E}_{0}(\infty)$. The pressure from $10<\mathrm{v}<$ largest value is fitted to a power function, which gives $\mathrm{C}, \omega$ and $\mathrm{E}_{0}$. The curve is extrapolated to infinity to get $E_{0}(\infty)$. Next, $A, B, R_{1}$ and $R_{2}$ are found by fitting the entire adiabat. The average error in the pressure and the energy is minimized. The variables $R_{1}$ and $R_{2}$ are varied and the $\mathrm{A}$ and $\mathrm{B}$ are calculated from the linear $\mathrm{C}-\mathrm{J}$ equations:

$$
\begin{aligned}
& \frac{1}{2} P_{c j}\left(1-v_{c j}\right)=\left(\frac{A}{R_{1}}\right) \exp \left(-R_{1} v_{c j}\right)+\left(\frac{B}{R_{2}}\right) \exp \left(-R_{2} v_{c j}\right)+\frac{C}{\omega v_{c j}^{\omega}}+E_{o} \\
& \rho_{0} D^{2}=A R_{1} \exp \left(-R_{1} v_{c j}\right)+B R_{2} \exp \left(-R_{2} v_{c j}\right)+\frac{(1+\omega) C}{v_{c j}^{(2+\omega)}}
\end{aligned}
$$

An example of the CHEETAH-generated JWL's, containing the $2145 \mathrm{~K}$ freeze, are in Mbar units:

\begin{tabular}{cccc} 
& PETN & LX-14 & LX-17 \\
\cline { 2 - 4 }$\rho_{\mathrm{o}}$ & 1.766 & 1.825 & 1.909 \\
$\mathrm{~A}$ & 8.6735 & 10.0004 & 8.6781 \\
$\mathrm{~B}$ & 0.0762 & 0.1052 & 0.0649 \\
$\mathrm{R}_{1}$ & 4.61 & 4.73 & 4.77 \\
$\mathrm{R}_{2}$ & 0.94 & 1.05 & 1.00 \\
$\omega$ & 0.33 & 0.35 & 0.27 \\
$\mathrm{E}_{\mathrm{o}}$ & 0.1086 & 0.1066 & 0.0835
\end{tabular}




$\begin{array}{cccc}\mathrm{D} & 0.852 & 0.876 & 0.773 \\ \Gamma_{\mathrm{j}}+1 & 4.144 & 4.127 & 4.225 \\ \mathrm{P}_{\mathrm{j}} & 0.309 & 0.340 & 0.270 \\ \mathrm{C} & 0.0151 & 0.0141 & 0.0094 \\ \mathrm{v}_{j} & 0.759 & 0.757 & 0.764\end{array}$

The average of 11 explosives gives

$$
\begin{aligned}
& R_{1}=4.69 \pm 0.10 \\
& R_{2}=1.01 \pm 0.04
\end{aligned}
$$

which is close to the results from the previous chapter. The CHEETAH JWL's are derived from previous cylinder JWL's so that they should be good but cannot provide independent information. The user should always check the coefficients closely, because CHEETAH sometimes diverges to give strange values.

\section{Fabry Dependence on the Velocity Angle}

The Cylinder Test has the wall perpendicular and the velocity pointing in different directions. In the previous report, we used a simple model in which the wall bends out like a hinge. ${ }^{6}$ The wall is always straight and has an angle $\Theta_{\mathrm{S}}$ to the original wall, as seen in this diagram. The detonation wave moves left to right at the bottom and the wall pushes out upward. The hinge-wall is the line $\mathrm{BC}$.

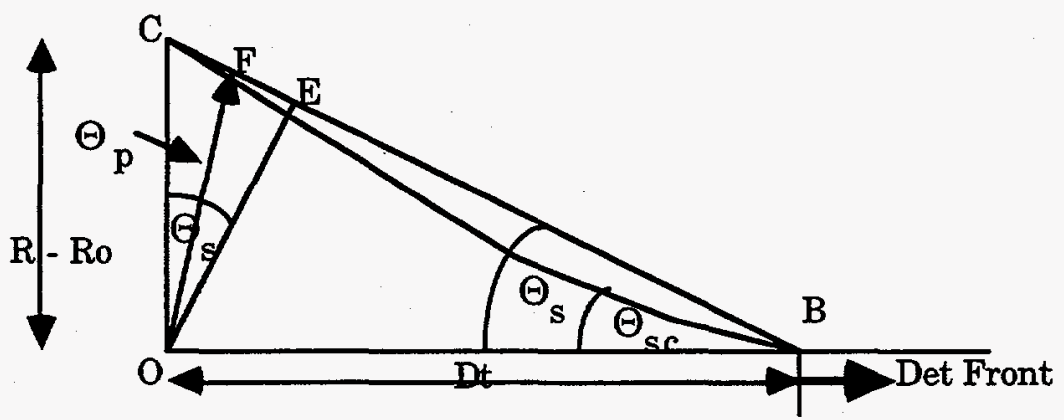

If this were true, then the wall angle as seen from the outer surface would be

$$
\Theta_{s}=\tan ^{-1}\left(\frac{R-R_{0}}{D t}\right)
$$


where $R-R_{0}$ is the measured streak camera distance (line OC above), $t$ is the time and $D t$ is the distance OB. As shown in Fig. 4-2, this grows to about $13^{\circ}$.

The expanding wall is actually bent as shown by the curved line $\mathrm{BC}$ above.

The instantaneous wall position may be again be calculated differentially from the streak camera distance to give

$$
\Theta_{s c}=\tan ^{-1}\left[\frac{\left(R-R_{o}\right)_{i+1}-\left(R-R_{o}\right)_{i}}{D\left(t_{i+1}-t_{i}\right)}\right]
$$

where the ith and $(i+1)$ st data points are used. This angle, which reaches $15^{\circ}$, is shown in Fig. $4-2$ with the HEMP code values, which are taken by hand-measuring snapshot views.

The wall velocity does not move normal to the wall's surface. We showed this simply before with the hinge model. ${ }^{6}$ The hinge wall swings out from $\mathrm{OB}$ to $\mathrm{OF}$ along an arc. The approximate path OF is different from the normal to the wall OE. We showed that the angle belonging to $O F, \Theta_{p}$, was equal to $\Theta_{\mathrm{sc}} / 2$. Because the hinge model is not quite right, the approximate velocity angle is

$$
\Theta_{p} \approx \frac{\Theta_{s c}}{2}=\frac{1}{2} \tan ^{-1}\left[\frac{\left(R-R_{o}\right)_{I+1}-\left(R-R_{o}\right)_{i}}{D\left(t_{i+1}-t_{i}\right)}\right]
$$

This allows the use of streak camera data. The result is shown in Fig. 4-2 along with the nodal vector angles from HEMP. The approximate velocity angle is $7^{\circ}$.

At one time, it was not clear whether the Fabry beam should be set to the velocity vector or the wall surface normal. This was studied experimentally by David Goosman, et. al., 7 who found that their Fabry-Perot velocimetry ${ }^{8}$ is sensitive only to the direction of the velocity, and not to the position of the surface. They fired aluminum projectiles at $1 \mathrm{~mm} / \mu$ s in a $19 \mathrm{~mm}$ diameter gun. The front of each projectile had a flat center face plus a $20^{\circ}$ or $45^{\circ}$ ramp face to the side. The fiducial projectile had a flat $0^{\circ}$ front face with the Fabry also hitting on a flat $90^{\circ}$ "ramp" side face of the projectile. The Fabry beam was bounced off the two approaching faces when the gun was fired. The expected measured velocity was calculated for dependence on the velocity only and on the position, i.e. the tilt of the surface. The results show that the velocity alone affects the Fabry result. 


\begin{tabular}{|c|c|c|c|c|}
\hline \multirow[b]{2}{*}{ Configuration } & \multicolumn{3}{|c|}{ Velocity $(\mathrm{mm} / \mu \mathrm{s})$} & \multirow[b]{2}{*}{$\begin{array}{l}\text { Sur- } \\
\text { face }\end{array}$} \\
\hline & $\begin{array}{c}\text { Calcd. } \\
\text { Velocity }\end{array}$ & $\begin{array}{c}\text { Calcd. } \\
\text { Position }\end{array}$ & $\begin{array}{l}\text { Exper- } \\
\text { iment }\end{array}$ & \\
\hline $90^{\circ}$ Incidence-flat & 0 & 0 & 0 & smooth \\
\hline $90^{\circ}$ Incidence-ramp & 0 & 2.00 & 0 & smooth \\
\hline $45^{\circ}$ Incidence-flat & 1.41 & 0 & $1.43 \pm 0.07$ & rough \\
\hline $45^{\circ}$ Incidence-ramp & 1.41 & $1 . \dot{4} 1$ & $1.43 \pm 0.07$ & rough \\
\hline $20^{\circ}$ Incidence-flat & 1.88 & 2.13 & $1.91 \pm 0.07$ & smooth \\
\hline $20^{\circ}$ Incidence-ramp & 1.88 & 2.45 & $\begin{array}{l}1.87 \pm 0.04 \\
1.86 \pm 0.06\end{array}$ & $\begin{array}{l}\text { rough } \\
\text { smooth }\end{array}$ \\
\hline
\end{tabular}

The error between the Fabry measurement and the velocity is the cosine of the difference between them.

Fig. 4-3 shows the velocity angle, $\Theta_{\mathrm{p}}$, for various explosives, as calculated from Eq. 10. In the $2<\mathrm{v}, 7$ region used for making JWL's, the angle is about $7^{\circ}$ for powerful explosives, which is the traditional angle the Fabry beam is set at. However, it appears that this angle could be as low as $5^{\circ}$ for weak explosives.

\section{Accuracy of the Cylinder Fabry Trace (with Dave Goosman)}

Consider a 1-inch half wall PETN of $1.76 \mathrm{~g} / \mathrm{cc}$ (old standard cylinder \#511). At an $R-R_{0}$ of 32 $\mathrm{mm}$, the time is about $15 \mu \mathrm{s}$ and the velocity about $2.5 \mathrm{~mm} / \mu \mathrm{s}$. We require that the record go at least to $R-R_{0}$ of $25 \mathrm{~mm}$ so that we amply pass the the cylinder-measuring distance of $19 \mathrm{~mm}$. This way, we have the three points we need to get the JWL. As of September, 1994, the slit widths were 0.05 $\mathrm{mm}$ and the slit image on the phosphor $0.15 \mathrm{~mm}$. The number of time resolution elements is the distance divided by the larger image or $32 / 0.15=213$. The resolution is $15 \mu \mathrm{s} / 213 \sim 70 \mathrm{~ns}$ or roughly, 1/250th of the record length. The shorter the record, the greater the resolution. The time fiducial markers (fids) have a 30 ns full-width-at-half-height, with an \pm 11 ns uncertainty in reading the position. We must read between two fids, which increases the error to $22 \mathrm{~ns}$ to which is added a $20 \mathrm{~ns}$ error due to uncertainty in jump-off. Then, the error in reading the time at $15 \mu \mathrm{s}$ is

$$
\text { time error } 0.042 / 15=0.28 \%
$$

There are two errors in considering the velocity. The first is the difficulty in resolving the laser interferometer fringes. The finesse is estimated to be 25 , where the finesse is the ratio of distance between fringes to the fringe width. The uncertainty is about $\pm 0.6 \mathrm{~mm} / \mu \mathrm{s} \cdot$ fringe. For 50 
fringes, we have $\pm 0.012 \mathrm{~mm} / \mu \mathrm{s}$. At the $2.5 \mathrm{~mm} / \mu \mathrm{s}$ velocity, this is an error of $\pm 0.5 \%$. There is a second error which has to do with spacing variations of the mirrors, which is estimated to be $\pm 0.25 \%$. The velocity error, assuming the worst case where they add in the same direction, is then

$\mathrm{u}$ error $\sim 0.012+\mathrm{u} / 400 \sim 0.018 \mathrm{~mm} / \mu \mathrm{s}$ or $\pm 0.73 \%$ at $2.5 \mathrm{~mm} / \mu \mathrm{s}$.

An even larger error occurs for the jump-off. If the laser spot size is $2 \mathrm{~mm}$ and the detonation velocity is $8 \mathrm{~mm} / \mu \mathrm{s}$, then the jump-off takes $0.25 \mu \mathrm{s}$ to move from one edge of the spot to the other. This uncertainty translates directly into the jump-off time, where the jump-off will rise linearly for the $0.25 \mu \mathrm{s}$. Once it tops out, if the velocity pull-back is $10 \%$, then a $5 \%$ error could be present by averaging across the first step. More error enters when the second jump occurs. The spot-size error decreases with time as the acceleration of the wall declines.

To get the distance, the Fabry velocity must be integrated.

$$
R-R_{o}=\int_{0}^{t_{f}}\left[u \pm 0.012 t \pm \frac{u}{400}\right] d t
$$

The overall time uncertainty for the upper limit of integration, $t_{f}$, is set at $\pm 33 \mathrm{~ns}$. This affects the final velocity by $2.5 \mathrm{~mm} / \mu \mathrm{s}(33 \mathrm{~ns}) \sim \pm 0.08 \mathrm{~mm}$. The velocity itself has the two components we mentioned above: the time-increasing part with the error $\pm 0.012 \mathrm{t}$ and the constant $1 / 4 \%$ error. The first is $0.012(15 \mu \mathrm{s}) \sim 0.18 \mathrm{~mm}$; the seond is $32 \mathrm{~mm} / 400 \sim 0.08 \mathrm{~mm}$. It is likely that the errors will partly cancel, but we will add up the worst case and get $0.08+0.18+0.08= \pm 0.34$ $\mathrm{mm}$ or $0.34 / 32$ or $\pm 1.1 \%$. We arrive at the long-time error for the integration of the Fabry as being about

$$
R-R_{0} \text { error } \sim \pm 1 \% \text {. }
$$

Soon a new timing system will come from EG\&G, which will sharpen the fids by a factor of 2 . A new laser fiber taper technique is expected to give 3 times more light. This is expected to cut in half the time uncertainty of $t_{f}$ and $2 / 3$ rds off the time-dependent error to $0.008 \mathrm{t}$. The quarter-\% mirror error remains, so that we now get

$$
\begin{aligned}
& \mathbf{u} \text { error } \sim 0.008+\mathrm{u} / 400 \sim 0.014 \mathrm{~mm} / \mu \mathrm{s} \text { or } \pm 0.57 \% \text { at } 2.5 \mathrm{~mm} / \mu \mathrm{s} . \\
& R-R_{0} \text { error } \sim 0.04+0.12+0.08= \pm 0.24 \mathrm{~mm} \text { or } \pm 0.75 \%
\end{aligned}
$$




\section{Accuracy of the Cylinder Streak Camera (with Raul Garza and Frank Helm)}

The expansion of the cylinder is captured on a large piece of film such that the initial outer diameter is always reduced to about 22 to $23 \mathrm{~mm}$. The starting cylinder is first photographed for perhaps a microsecond with movie studio lights to produce the "still" film portion. Then the argon candles (about 6 lbs of pyrotechnics) are lit and the original cylinder is photographed for several $\mu \mathrm{s}$ and the static film portion is taken. The test begins and the cylinder expands, using the candles as illumination of the edges. The rotor speed of the film driver is known accurately. The largest error comes from the $25 \mu \mathrm{m}$-wide slit through which the light comes in to hit the film. The image on the film is doubled to $50 \mu \mathrm{m}$ so that the error in position caused by the slit is $\Delta \mathrm{x} \sim 25 \mu \mathrm{m}$. For a writing speed on the film, $u_{w}$, we have a time uncertainity of $\Delta x / u_{w}$. This times the wall velocity, $u$, gives a distance uncertainty, which may be compared with the total distance, $R-R_{0}$. The error is then

$$
\% \text { distance error }=100 \frac{\mathrm{u}}{\mathrm{u}_{\mathrm{w}}} \frac{\Delta \mathrm{x}}{\mathrm{R}-\mathrm{R}_{\mathrm{o}}} \text {. }
$$

Below, we list various writing speeds used recently, which vary from 0.1 to $5 \mathrm{~mm} / \mu \mathrm{s}$. Data from $1.76 \mathrm{~g} / \mathrm{cc}$.PETN (shot \#586) is used for illustration. We have:

\begin{tabular}{ccc|cccc} 
& & & \multicolumn{5}{|c}{$\begin{array}{c}\text { distance error at these writing } \\
\text { scaled }\end{array}$} & $\mathrm{u}$ & $\mathrm{R}-\mathrm{R}_{0}$ & \multicolumn{4}{|c}{ speeds $(\mathrm{mm} / \mu \mathrm{s})$} \\
\cline { 4 - 7 } $\mathrm{t}(\mu \mathrm{s})$ & $(\mathrm{mm} / \mu \mathrm{s})$ & $(\mathrm{mm})$ & 0.1 & 0.5 & 1 & 5 \\
\hline 5 & 2.2 & 9 & 6.1 & 1.2 & 0.6 & 0.12 \\
10 & 2.4 & 20.5 & 2.9 & 0.6 & 0.3 & 0.06 \\
15 & 2.5 & 33 & 1.9 & 0.4 & 0.2 & 0.04
\end{tabular}

We see that the error drops as $R-R_{0}$ and the writing speed increase. In prectice, the accuracy of a single streak camera distance could be very good. We now look at actual recent cylinder shots and measure the precision of the four camera traces, two on each side. With time in 1-inch-scaled seconds, the data is as follows:

\begin{tabular}{c|ccc|ccc|c} 
& \multicolumn{3}{|c|}{$\begin{array}{c}\text { Standard } \\
\text { Deviation (mm) at }\end{array}$} & \multicolumn{3}{c|}{$\begin{array}{c}\mathrm{R}-\mathrm{R}_{\mathrm{o}} \\
\text { \% } \mathrm{R}_{\mathrm{o}} \text { at }\end{array}$} & $\begin{array}{c}\text { at } 15 \mu \mathrm{s} \\
\text { at }\end{array}$ \\
\cline { 2 - 8 } & $5 \mu \mathrm{s}$ & $10 \mu \mathrm{s}$ & $15 \mu \mathrm{s}$ & $5 \mu \mathrm{s}$ & $10 \mu \mathrm{s}$ & $15 \mu \mathrm{s}$ & $(\mathrm{mm})$ \\
\hline pm547 & 0.12 & 0.16 & 0.20 & 0.7 & 0.4 & 0.3 & 64 \\
pm554 & 0.03 & 0.05 & 0.12 & 0.3 & 0.2 & 0.3 & 41 \\
pm548 & 0.05 & 0.06 & 0.08 & 0.5 & 0.3 & 0.2 & 37
\end{tabular}




\begin{tabular}{c|ccc|ccc|c} 
pm564 & 0.12 & 0.17 & 0.22 & 1.2 & 0.7 & 0.6 & 36 \\
pm549 & 0.11 & 0.20 & 0.26 & 1.1 & 0.9 & 0.7 & 35 \\
pm586 & 0.07 & 0.13 & 0.22 & 0.8 & 0.7 & 0.7 & 33 \\
pm583 & 0.04 & 0.08 & 0.14 & 0.5 & 0.4 & 0.4 & 30 \\
pm585 & 0.02 & 0.05 & 0.09 & 0.2 & 0.3 & 0.3 & 27 \\
pm580 & 0.05 & 0.10 & 0.15 & 0.7 & 0.6 & 0.6 & 25 \\
pm574 & 0.08 & 0.08 & 0.08 & 1.6 & 0.7 & 0.4 & 18 \\
pm575 & 0.09 & 0.09 & 0.09 & 1.8 & 0.8 & 0.5 & 18 \\
pm573 & 0.01 & 0.01 & 0.01 & 0.3 & 0.1 & 0.1 & 18 \\
pm581 & 0.04 & 0.13 & 0.01 & 1.2 & 1.6 & 0.1 & 13 \\
pm582 & 0.04 & 0.13 & 0.02 & 1.1 & 1.6 & 0.2 & 13 \\
\hline Average & 0.06 & 0.10 & 0.12 & 0.8 & 0.7 & 0.4 &
\end{tabular}

(20)

The standard deviation is independent of the strength of the explosive as expected (LX-14 about $60 \mathrm{~mm}$ at $15 \mu \mathrm{s} ; \mathrm{LX}-17$ about $40 \mathrm{~mm}$ ). The streak distance \% error is \pm 0.4 to $\pm 0.8 \%$ on the average; for LX-14, with care, it might be cut in half. Below are the approximate numbers for the Fabry integration using the equipment as it presently exists. The Fabry is closing on the streak camera as a means of determining distance. This is important because of the cost of streak cameras. It appears that, within a few years, they may not be needed.

We consider other aspects of the measurement. The film is read on a Grant Comparator, an optical film reader with precise stepping motors. ${ }^{9}$ The stage travel is listed in the specifications as being 4 inches $\mathrm{X}$-axis and 18 inches $\mathrm{Y}$-axis. The "positioning resolution" is listed as $\pm 1 \mu \mathrm{m}$. The width of the wall on the photograph is estimated as being about $5 \mu \mathrm{m}$. The precision of measurement appears to be about \pm 10 to $\pm 20 \mu \mathrm{m}$. For $R-R_{0} \sim 20$ photo $\mathrm{mm}$, we would have a maximum measuring error of $\pm 0.2 \%$. This could limit whatever gains are made in more careful measurement.

As a further check for this report, a Bausch \& Lomb transparent eyeloop scale was placed directly in the Grant Comparator for calibration. The results show that the equipment is in calibration. 


\begin{tabular}{ccccc} 
& \multicolumn{4}{c}{ Distance $(\mu \mathrm{m})$} \\
\cline { 2 - 4 } & $\begin{array}{c}\text { Eyeloop } \\
\text { Scale }\end{array}$ & $\begin{array}{c}\text { Grant } \\
\text { Scale }\end{array}$ & $\begin{array}{c}\text { Diff- } \\
\text { erence }\end{array}$ & $\%$ \\
\hline Hori- & 100 & 96 & 4 & 4 \\
zontal & 500 & 498 & 2 & 0.4 \\
& 1000 & 997 & 3 & 0.3 \\
& 20000 & 19999 & 1 & 0.005 \\
\hline Vert- & 100 & 97 & 3 & 3 \\
ical & 500 & 500 & 0 & 0 \\
& 1000 & 1001 & -1 & -0.1 \\
& 20000 & 19999 & 1 & 0.005
\end{tabular}

The manual control knob was also checked and found to be $5 \mu \mathrm{m}$ per click, a small increment.

One issue that does not matter is the difference between the still and static diameters, which can have either sign and averages about $\pm 0.35 \%$. The static and dynamic measurements, made with the candle light, are better than the studio shot, which is taken as a beginning reference only.

The velocity is obtained by differentiation of the streak record. The noise obtained without smoothing is about $\pm 0.1 \mathrm{~mm} / \mu \mathrm{s}$. For a $2 \mathrm{~mm} / \mu \mathrm{s}$ wall velocity, this is $\pm 5 \%$. How far we may go in smoothing is debatable, but halfway seems reasonable, so that

$$
\text { velocity error } \sim \pm 0.05 \mathrm{~mm} / \mu \mathrm{s} \sim \pm 2.5 \%
$$

and

$$
\text { detonation energy error } \sim \pm 5 \% \text {. }
$$

This is one source of the estimate that detonation energies that we slosh around in the JWL's are good to $5 \%$.

\section{Simple Theory of the Cylinder}

We shall try a simple theory of the cylinder based on the old work of $\mathrm{Neal}^{10}$ and the results of the HEMP code runs. This is the case where the geometric angle, $\alpha$, is $90^{\circ}$. Neal assumed that the sideways impact bent the wall out at an angle $\gamma$, thereby creating a gap with new volume. He also assumed that the pressure in the explosive and in the metal were the same, i.e. that there is no reflected wave. If the containment were perfect and no gap was created, then the pressure at the 
wall would be the detonation pressure. Because new volume is created in the gap, the actual pressure is lower. For copper, he got a pressure that was about $2 \mathrm{P}_{\mathrm{j}} / 3$.

Below is the schematic for the cylinder problem.

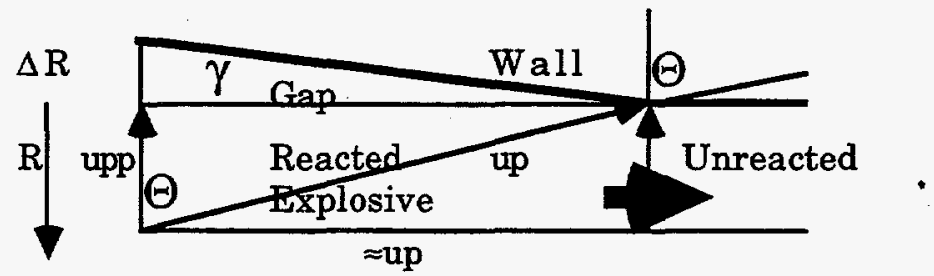

The explosive is detonating left-to-right with the wave velocity, $D$. The mass flow, $\mathrm{u}_{\mathrm{p}}$, near the wall hits it at an angle $\Theta$. This should be reflected in the zone nearest the wall in the code. Because $\Theta$ is close to $90^{\circ}$, the length along the cylinder axis is almost $u_{p}$ and the perpendicular vector into the wall, upp, is

$$
u_{p p}=u_{p} \cos \Theta
$$

Also defined in Eq. 24 is the initial explosive radius $R$ and the increase in radius caused by the gap, $\Delta R$. To relate distance to velocity, we need a time for the "instant of detonation", $\Delta t$. The initial volume of the ring of explosive involved in the "instant", $\mathrm{V}$, is

$$
V \approx \pi u_{p} \Delta t\left[R^{2}-\left(R-u_{p p} \Delta t\right)^{2}\right]
$$

The new volume created by moving the wall is

$$
\Delta V=\frac{1}{2} \pi u_{p} \Delta t\left\{\left[R+\left(u_{p} \Delta t\right) \tan \gamma\right]^{2}-R^{2}\right\}
$$

The detonation relative volume, $\mathrm{vj}$, is modified to the new explosive volume, $v_{e}$, by

$$
\begin{aligned}
v_{e} \approx\left(\frac{V+\Delta V}{V}\right) v_{j} & =\left\{1-\frac{u_{p} \tan \gamma}{2 u_{p p}}\left(\frac{2 R+u_{p} \tan \gamma \Delta t}{2 R-u_{p p} \Delta t}\right)\right\} v_{j} \\
& =\left\{1-\frac{u_{p} \tan \gamma}{2 u_{p p}}\right\} v_{j} .
\end{aligned}
$$


From Appendix $B, \Delta t \approx 0.05 \mu$ s. If we ignore the last terms, we remove $\Delta t$ from the result.

For LX-14, the transverse jump-off velocity derived from the measured free surface velocity is about $0.5 \mathrm{~mm} / \mu \mathrm{s}$. The detonation velocity is $8.8 \mathrm{~mm} / \mu \mathrm{s}$, so that

$$
\gamma=\sin ^{-1}\left(\frac{u_{m}}{D}\right)=\sin ^{-1}\left(\frac{0.5}{8.8}\right) \approx 3.3^{\circ}
$$

where $u_{m}$ is the jump-off velocity of the inner, confined face of the metal plate. It is usually experimentally taken to be one-half the measured velocity of the unconfined outer face. This is true as long as the plate is thin. For LX-14 in Eq. $28, v_{j}=0.735, u_{p p}=u_{m}=0.5 \mathrm{~mm} / \mu$ s and $u_{p}=2.3$ $\mathrm{mm} / \mu \mathrm{s}$, as determined from $\mathrm{v}_{\mathrm{j}}$ and the detonation velocity. Then, we obtain $\mathrm{v}_{\mathrm{e}} \approx 0.87$ and from the $\mathrm{A}=7.65 \mathrm{JWL}, \mathrm{Pe}_{\mathrm{e}} \approx 23 \mathrm{GPa}$, about $62 \%$ of the detonation pressure. We note that we have made another important assumption: that the particle velocities of the explosive and the metal perpendicular to the initial wall are the same for the cylinder.

It would be nice to be able to calculate $u_{m}$ without measuring it first. This can be done for the cylinder by assuming, as Neal did, that the explosives and metal pressures at the interface are equal. 10 Then, we use the mass/momentum condition

$$
P_{e}=P_{m}=\rho_{m} U_{m} u_{m}
$$

which becomes

$$
\rho_{m} S_{1} u_{m}^{2}+\rho_{m} C_{o} u_{m}-P_{m}=0
$$

For copper, the $U_{\mathrm{s}}-u_{\mathrm{p}}$ coefficients are: $\mathrm{C}_{0} 3.94 \mathrm{~mm} / \mu \mathrm{s}$ and $\mathrm{S}_{1}$ 1.489.11 Then

$$
u_{m}=\frac{1}{2 \rho_{m} S_{1}}\left\{-\rho_{m} C_{o}+\left[\left(\rho_{m} C_{o}\right)^{2}+4 \rho_{m} S_{1} P_{m}\right]^{1 / 2}\right\}
$$

The problem may be solved by running a set of estimated angles, $\gamma$, through the process until $u_{m}$ is calculated, which, from Eq. 29, produces $\gamma$ again. The answer occurs where the two $\gamma$ 's are the same. For LX-14, we get: $\mathrm{u}_{\mathrm{m}}=0.575 \mathrm{~mm} / \mu \mathrm{s}, \gamma=3.75^{\circ}, \mathrm{P}_{\mathrm{e}}=24.6 \mathrm{GPa}$ and $\mathrm{v}_{\mathrm{e}}=0.848$. The theory contains no chemistry and assumes that the explosive is capable of detonating at the low value of $25 \mathrm{GPa}$. 
Finally, we calculate the angle $\Theta$ from Eq. 25 and obtain $\cos ^{-1}(0.575 / 2.3) \approx 76^{\circ}$. We recall that this is about the angle of intersection of the detonation front measured at the end of the cylinder for HMX and TATB with copper, although there we used the $90^{\circ}$ complement to the angle of $14^{\circ} .12$ We note that $\Theta$ is different from the expected geometric angle of incidence of $90^{\circ}$.

The HEMP code picture of the cylinder detonation front is shown in Fig. 4-4 for LX-14 described by a JWL. The explosive is at the bottom and the copper at the top. The detonation moves from left to right. Across most of the cylinder, the detonation front is perpendicular to the wall and the nodal vectors are normal. We see the part next to the wall. There is a slight bend in the pressure contour and the nodal vectors are turned to $15^{\circ}$. The pressure in the explosive is a maximum of 25 $\mathrm{GPa}$, about $2 / 3 \mathrm{rds}$ the detonation pressure. There is no reflected shock wave in the explosive. The refracted shock wave in the copper is evident and the nodal vectors are turning towards being perpendicular to the outer wall of the copper.

\section{Jump-Off Velocities for the Cylinder and the Plate}

We now summarize the cylinder jump-off velocities as compared with comparable data from the electric gun 1-D plate shots. Also included are the Sideways Plate Push results. 13 This new test, developed by Craig Tarver, is shown below schematically:

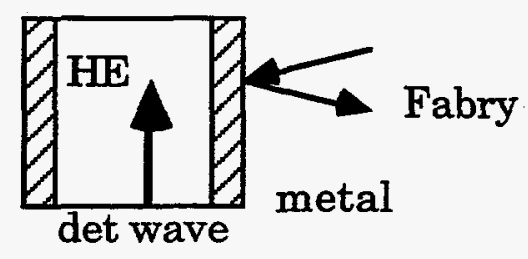

The sample is a planar sandwich. The center is a rectangular solid of $\mathrm{HE}$ of $12.7 \mathrm{~mm}$ length and width and $51 \mathrm{~mm}$ height. The shaded areas are the metal, either $\mathrm{Cu}$ or $\mathrm{Ta}, 20$ mils $(0.51 \mathrm{~mm})$ thick. The detonation wave comes from the bottom and is triggered by the 10 -mil mylar flyer at $4.3 \mathrm{~mm} / \mu \mathrm{s}$ from the electric gun. The flyer is used bare on LX-14 but an LX-10 booster is used in front of the LX-17. The Fabry beam looks at the transverse wall motion about $2 / 3$ rds of the way up the metal plate.

The 1-D electric gun data for LX-14 is extrapolated back from the thicker plates, so that we are using the zero-thickness velocity without any spike effects being present. LX-17 is always in the reaction zone and we have no choice but to extrapolate back to zero-thickness. Both transverse experiments have thicker plates which cannot be corrected back to zero thickness, so that these 
jump-off values are several percent low because of losses in the plate. All the data, with averages, is assembled in Table 4-1. We see that the cylinder jump-off is about $1 / 2$ that of the straight-ahead plate shot. This will be further developed in the next chapter.

\section{References}

1. P. C. Souers and L. C. Haselman, Jr., Detonation Equation of State at LLNL, 1993, LLNL Report UCRL-ID-116113 (March, 1994), pp. 5-5 and 5-6.

2. Laurence E. Fried, CHEETAH 1.0 User's Manual, LLNL Report UCRL-MA-117541 (1994).

3. L. E. Fried and P. C. Souers, CHEETAH: A Next Generation Thermochemical Code, LLNL Report UCRL-ID-117240 (1994).

4. L. E. Fried and P. C. Souers, "BKWC: An Empricial BKW Paramatrization based on Cylinder Test Data, Propellants, Explosives, Pyrotechnics, to be published.

5. M. L. Hobbs and M. R. Baer, "Calibrating the BKW-EOS with a Large Product Species Data Base and Measured C-J Properties," 10th International Detonation Symposium. Boston, MA, to be published.

6. Reference 1, pp. 4-2 to 4-4, 4-8.

7. D. R. Goosman, A. M. Frank, H. H. Chau and N. L. Parker, "Fabry-Perot Velocimetry Techniques: Is Doppler Shift affected by Surface Normal Direction?", High Speed Photography, Videography, and Photonics, Proceedings International Society for Optical Engineering (SPIE), San Diego, CA, August 23-25, 1983, 427, 127 (1983).

8. C. F. McMillan, D. R. Goosman, N. L. Parker, L. L. Steinmetz, H. H. Chau, T. Huen, R. K. Whipkey and S. J. Perry, Rev. Sci. Instruments $\underline{59} 1$ (1988).

9. Grant Instruments, Berkeley, CA, made in the early 1980 's.

10. T. Neal, "Perpendicular Explosive Drive and Oblique Shocks," Proceedings Sixth Symposium (International) on Detonation, Cororado, CA, August 24-27, 1976, p. 602.

11. D. J. Steinberg, Equation of State and Strength Properties of Selected Materials, LLNL Report UCRL-MA-106439 (1991).

12. Reference 1, p. 7-2.

13. C. M. Tarver, W. C. Tao and C. G. Lee, "Sideways Plate Push Test for Detonating Solid Explosives," Propellants, Explosives, Pyrotechnics, to be published. 


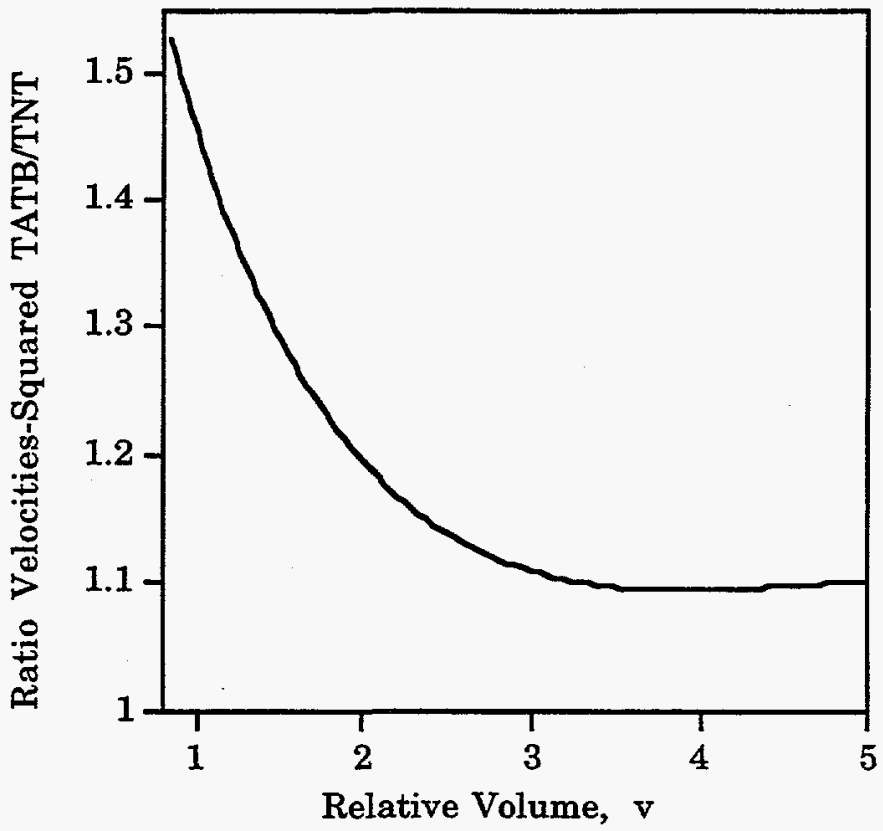

Fig. 4-1. Fitted ratio of cylinder wall velocities-squared for TATB as compared to TNT from the detonation velume $\mathrm{v}=0.85$ to $\mathrm{v}=5$.

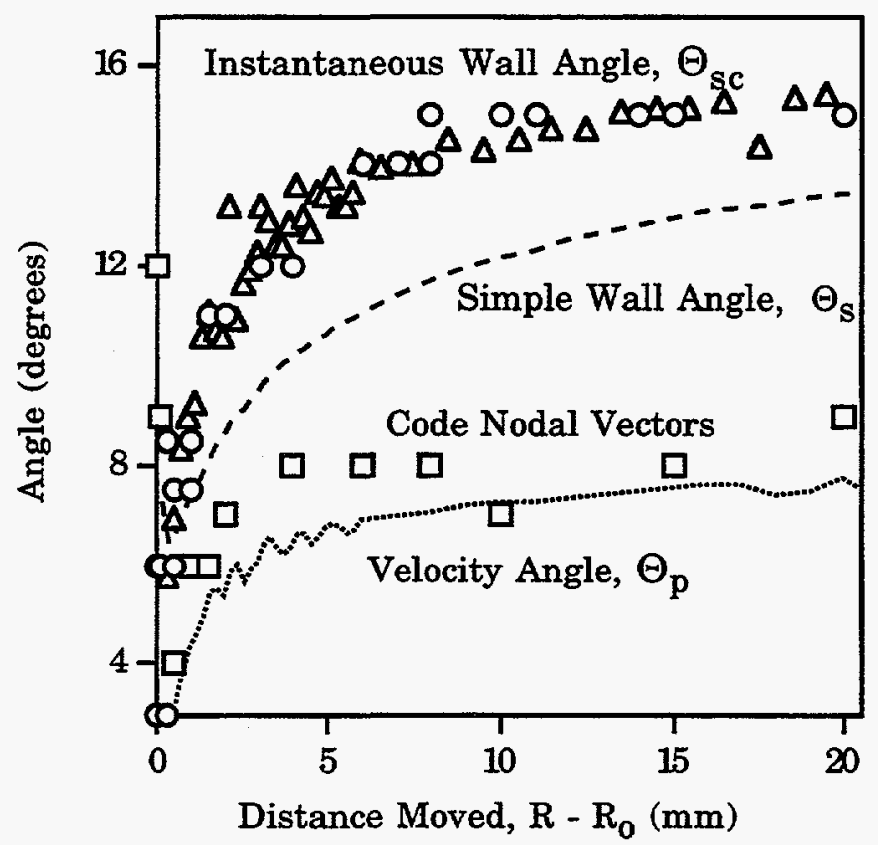

Fig. 4-2. Angles in the Cylinder Test as demonstrated with LX-14. The instantaneous wall angle, $\Theta_{\mathrm{Sc}}$, may be calculated from streak camera data $(\Delta$ ) or the HEMP code ( 0 ). The simple wall angle, $\Theta_{\mathrm{S}}$, is a simple approximation of the same thing but is low by about $2^{\circ}$. The wall velocity has a different angle, as shown by the code's nodal vectors $(\square)$. The dotted line shows the velocity angle, $\Theta_{\mathrm{p}}$, calculation. 


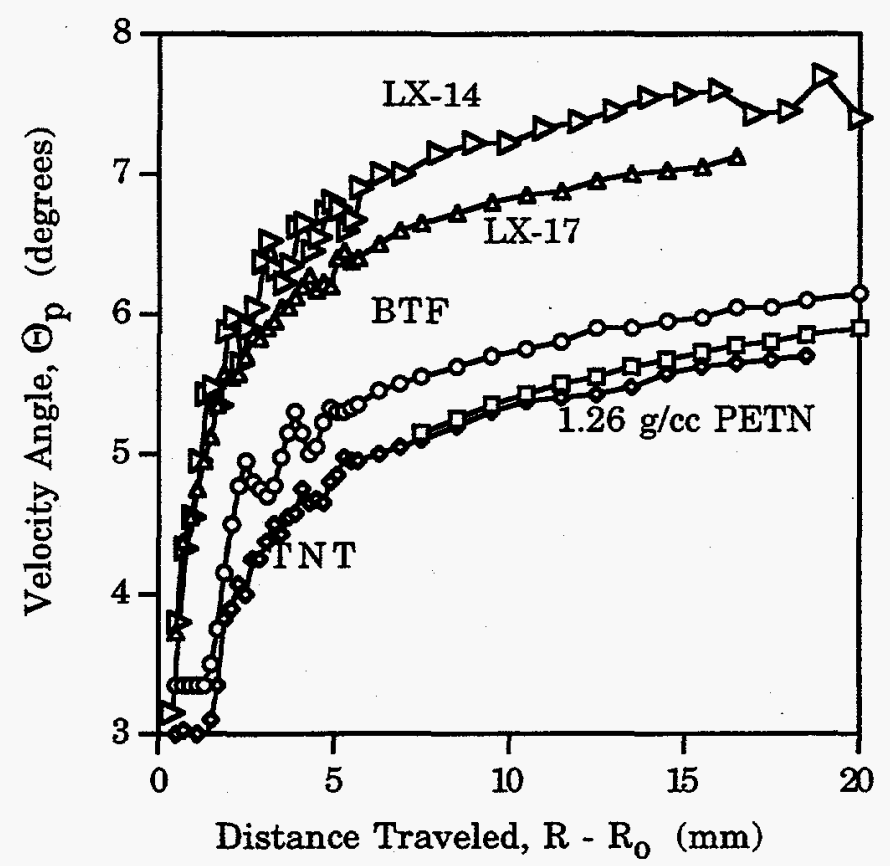

Fig. 4-3. Approximate cylinder velocity angles, $\Theta_{p}$, as calculated from streak camera results.

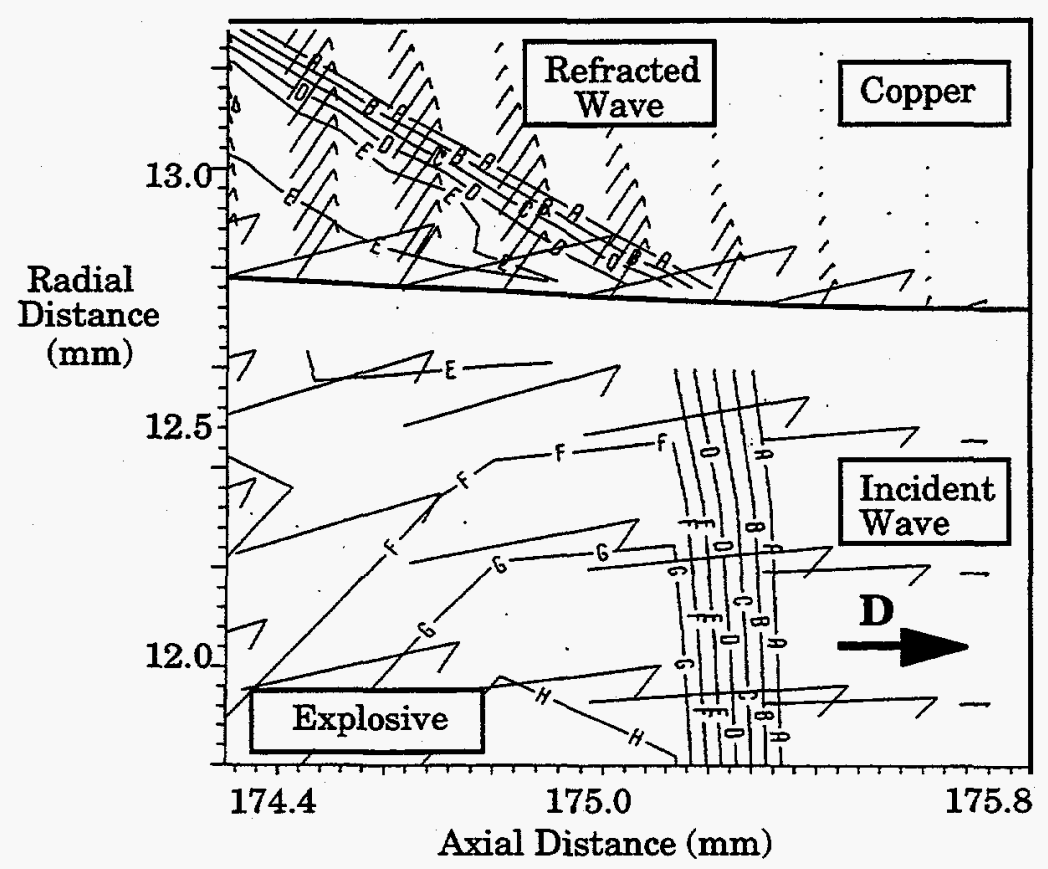

Fig. 4-4. HEMP view of the cylinder instant of impact at steady state. The detonation moves left to right. The LX-14 is below and the copper above. The detonation wave in the explosive is almost normal to the wall, but the nodal vector at the inner wall face is $75^{\circ}$. No reflected shock wave is seen. The node vectors in the copper begin to turn toward being perpendicular to the free surface. 
Table 4-1. Detailed list of comparative LLNL jump-off velocity data for straight-head and transverse shock wave interaction with a metal plate. The velocity is the measured free surface velocity, $u_{f s}$, and must be dividided by 2 to give the approximate inner face velocity, $u_{m}$. The three methods reported here are the 1-D electric gun, the cylinder test and the new sideways plate push. The boldface are the averaged results.

\begin{tabular}{|c|c|c|c|c|c|c|c|c|}
\hline \multirow{4}{*}{$\begin{array}{l}\text { Explo- } \\
\text { sive }\end{array}$} & \multirow[b]{4}{*}{ Metal } & \multirow{3}{*}{$\begin{array}{c}\text { Forward } \\
\text { Plate }\end{array}$} & \multirow[b]{3}{*}{ Cyl } & \multirow{4}{*}{$\begin{array}{c}\text { Sideways } \\
\text { Plate } \\
\text { No. }\end{array}$} & \multicolumn{4}{|c|}{ Jump-Off Velocity (mm/ $/ \mathrm{s})$} \\
\hline & & & & & \multirow{2}{*}{$\begin{array}{l}\text { For- } \\
\text { ward }\end{array}$} & \multicolumn{2}{|c|}{ Transverse } & \multirow{3}{*}{$\begin{array}{c}\text { Sideways } \\
\text { Plate }\end{array}$} \\
\hline & & & & & & Cyl & Cyl & \\
\hline & & Nos. & No. & & Plate & streak & Fabry & \\
\hline \multirow[t]{5}{*}{ LX-14 } & $\mathrm{Cu}$ & phm & 469 & phm94001 & & 1.0 & & 1.043 \\
\hline & & series & 510 & phm94002 & & 1.1 & & 1.05 \\
\hline & & 19 & 520 & & & 1.1 & & \\
\hline & & samples & 521 & & & 1.2 & 0.95 & \\
\hline & & Ave. & & & 2.2 & & & 1.01 \\
\hline \multirow[t]{3}{*}{$\mathrm{LX}-14$} & $\mathrm{Ta}$ & phm- & & phm93013 & & & & 0.85 \\
\hline & & $\begin{array}{c}17 \\
\text { samnles }\end{array}$ & & phm93014 & & & & 0.79 \\
\hline & & Ave. & & & 1.65 & & & 0.82 \\
\hline \multirow[t]{5}{*}{ LX-17 } & $\mathrm{Cu}$ & pta- & 470 & phm94003 & & 0.85 & & 0.95 \\
\hline & & series & 471 & phm94004 & & 0.9 & & 0.98 \\
\hline & & 17 & 522 & & & 1.0 & 0.8 & \\
\hline & & samples & 523 & & & 1.15 & $0.91,1.03$ & \\
\hline & & Ave. & & & 2.1 & & & 0.95 \\
\hline \multirow[t]{3}{*}{ LX-17 } & $\mathrm{Ta}$ & pta85001 & 554 & pta92062 & & 0.8 & 0.65 & 0.71 \\
\hline & & pta85002 & & pta93004 & & & & 0.745 \\
\hline & & Ave. & & & 1.65 & & & 0.70 \\
\hline
\end{tabular}




\section{Chapter 5. Bigplate}

\section{Bigplate: a Oblique Angle EOS Test}

Bigplate consists of a flat disc of explosive, $100 \mathrm{~mm}$ in radius and $20 \mathrm{~mm}$ thick. A schematic is shown in Fig. 5-1 with the list of angles and rays needed for the description shown in Fig. 5-2. In this case, the explosive is LX-14. A $0.5 \mathrm{~mm}$-thick copper plate is glued to it. The copper was probably cut from an unannealed sheet and received no special attention. A point detonator sits in the center of the explosive at the back, so that the detonation spreads outward and creates a 2-dimensional burn. To date, three LX-14 shots, 891-D1, 2 and 3 have been fired. The engineer was Al Watanabe and the ramrods were Jim Janzen and Larry Simmons. Shots 1 and 2 was $1.828 \mathrm{~g} / \mathrm{cc} \mathrm{LX-14}$ and shot 3 was $1.808 \mathrm{~g} / \mathrm{cc}$ explosive. Five Fabry beams hit the outside edge of the plate. In terms of the radial distance, the beams were set to hit at $-10,0,5,10$ and $20 \mathrm{~mm}$. The angles to the horizontal, $\varepsilon$, of these beams were $6^{\circ}, 0^{\circ}, 3^{\circ}, 6^{\circ}$ and $8^{\circ}$ and were set by the physical sizes of the diagnostic parts. In $891 \mathrm{D} 1$, one long Fabry ran for over $15 \mu \mathrm{s}$, giving us a time scale comparable to the cylinder test. In 891D2 and 891D3, two Fabrys ran for 2 and $5 \mu$ s each. The Fabry measurements were done by Rex Avara.

The geometric angle of incidence, $\alpha$, is the angle between the axis and the ray to some radius off-axis. On the axis of symmetry, the detonation runs $20 \mathrm{~mm}$ perpendicular to the plate. For the point where the Fabry hits at the radial distance $y$, the detonation wave runs down the hypotenuse. The time difference for the shock wave reaching the plate is

$$
\Delta t=\frac{2}{D}\left[1-\frac{1}{\cos \alpha}\right]
$$

This difference should appear in the starts of the Fabry traces. We use the slow Fabry traces, where the relative time uncertainty between two points on different curves is listed as $\pm 0.1 \mu \mathrm{s}$.

$$
y-
$$
position

Time Difference, $\Delta \mathrm{t}(\mu \mathrm{s})$

\begin{tabular}{cccc}
\cline { 2 - 4 }$(\mathrm{cm})$ & Calcd & $891 \mathrm{D} 2$ & $891 \mathrm{D} 3$ \\
\hline 0.00 & 0.00 & 0.00 & 0.00 \\
0.50 & 0.07 & 0.09 & 0.08 \\
-1.00 & 0.26 & 0.26 & 0.29 \\
1.00 & 0.26 & 0.25 & 0.24 \\
2.00 & 0.90 & 0.96 & 0.95
\end{tabular}


Within error, we find that the lighting time approach fits, in which we imagine the detonation velocity to immediately reach the steady state value of $8.8 \mathrm{~mm} / \mu \mathrm{s}$ and to proceed straight along a ray.

The most interesting data is the jump-off velocity of the copper plate measured at different distances off-axis. The jump-off velocity decreases the farther we move out.

\begin{tabular}{|c|c|c|c|c|c|c|}
\hline \multirow{2}{*}{$\begin{array}{c}\text { Radial } \\
\text { Distance } \\
(\mathrm{mm})\end{array}$} & \multirow{2}{*}{$\begin{array}{c}\text { Angle } \\
\alpha \\
\text { (degrees) }\end{array}$} & \multicolumn{2}{|c|}{ Jump-Off Velocity } & \multicolumn{2}{|c|}{ Pull-back Minimum } & \multirow{2}{*}{$\begin{array}{c}\text { Jump- } \\
\text { Off } \\
\text { Ratios }\end{array}$} \\
\hline & & 891D2 & $891 \mathrm{D3}$ & 891D2 & 891D3 & \\
\hline 0 & 0.0 & 1.85 & 1.81 & 1.71 & 1.65 & 1.000 \\
\hline 5 & 13.7 & 1.81 & 1.80 & 1.68 & 1.64 & 0.987 \\
\hline 10 & 26.0 & 1.83 & 1.71 & 1.58 & 1.57 & 0.952 \\
\hline 20 & 44.3 & 1.59 & 1.59 & 1.45 & 1.45 & 0.866 \\
\hline
\end{tabular}

Also, we consider the density difference of $0.02 \mathrm{~g} / \mathrm{cc}$ between samples 891D1\&2 and 891D3. These samples were carefully made to test whether the JWL's should be modified for density. Typical results are shown in Fig. 5-3 for data taken on-axis and $20 \mathrm{~mm}$ off. No significant difference as a result of the densities could be seen at these or at the three other positions.

\section{Simple Model of Oblique Contact with a Wall}

There remains the more general problem lying between the straight-ahead and transverse collision considered in Chapter 3. 1 The schematic below illustrates a case with a linear rod of explosive being detonated upward into a metal plate with an angle of incidence $\alpha$. The gap is being created at the left with the angle $\gamma$. The mass vector is again moving into the wall with an angle $\Theta$ that is different from $\alpha$.

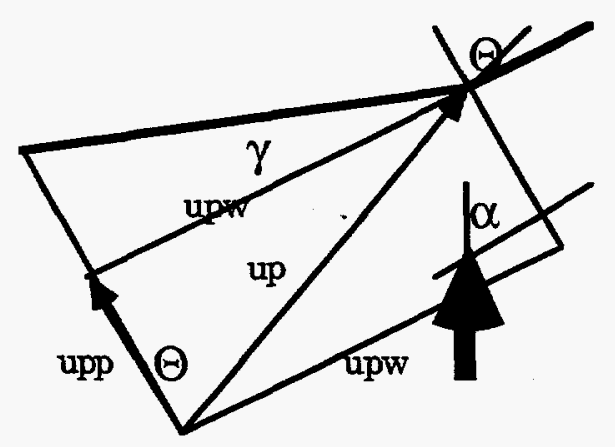


The 2-D volume of initial explosive that participates in the instant of detonation is $u_{p p} u_{p w} \Delta t^{2}$. Because $u_{p p}=u_{p} \cos \Theta$ and $u_{p w}=u_{p} \sin \Theta$, the volume becomes $u_{p}{ }^{2} \sin \Theta \cos \Theta \Delta t^{2}$. The volume added on by wall motion is $\left(u_{p w} \Delta t\right)^{2} \tan \gamma / 2$, which equals $u_{p}{ }^{2} \sin 2 \Theta \tan \gamma / 2$. Then

$$
\mathrm{v}_{\mathrm{e}}=\left(1+\frac{\Delta \mathrm{V}}{\mathrm{V}}\right) \mathrm{v}_{\mathrm{j}}=\left(1+\frac{\tan \Theta \tan \gamma}{2}\right) \mathrm{v}_{\mathrm{j}}
$$

which leads to the calculation of $P_{e}$ by inserting $v_{e}$ into a JWL. Also, the full Snell's Law for the metal particle vector is

$$
\gamma=\sin ^{-1}\left(\frac{u_{m}}{D} \sin \alpha\right)
$$

For an oblique angle, the pairs $P_{m}, P_{e}$ and $u_{e}, u_{m}$ are not equal.

We take the impedance equations derived in Appendix B from Deal ${ }^{2}$ for a plate hit straighton and correct for the angle. We write

$$
P_{m}=P_{e}+\rho_{r} D_{r}\left(u_{p p}-u_{m}\right)=P_{e}+\rho_{0} D \cos \alpha\left(u_{p} \cos \Theta-u_{m}\right)
$$

The same assumption is used to convert the reflected shock wave into terms of the initial explosive. The cosine associated with $\mathrm{D}$ assumes that the reflected and incident waves have the same angle. The two particle velocities being compared are perpendular to the metal wall. The explosive velocity is related back to the total velocity.

We next use the Rankine-Hugoniot explosive momentum/mass conservation corrected for D and $u_{p}$ having different directions. This is

$$
P_{e}=\rho_{0} D u_{p} \cos (\alpha-\Theta)
$$

For the metal, the usual $P_{m}=\rho_{0} U_{m} u_{m}$ still holds. We now solve for $u_{m}$ to get:

$$
\rho_{m} S_{1} u_{m}^{2}+\left(\rho_{m} C o+\rho_{0} D \cos \alpha\right) u_{m}-P_{\ell}\left[1+\frac{\cos \alpha \cos \Theta}{\cos (\Theta-\alpha)}\right]=0 .
$$


The first thought is that $\alpha=\Theta$ and the $P_{e}$ term becomes $1+\cos ^{2} \alpha$. Our simple cylinder problem in Chapter 4 gave $\Theta=75^{\circ}$ for $\alpha=90^{\circ}$. We shall further consider this below, but for now, we take

$$
\begin{array}{ll}
\Theta=\alpha, & 0^{\circ}<\alpha<75^{\circ} \\
\Theta=75^{\circ}, & \alpha>75^{\circ} .
\end{array}
$$

The calculation is summarized with these LX-14/copper results:

\begin{tabular}{cccccc}
$\begin{array}{c}\text { Angle } \\
\alpha(\mathrm{deg})\end{array}$ & $\begin{array}{c}\text { Angle } \\
\Theta(\mathrm{deg})\end{array}$ & $\begin{array}{c}\mathrm{u}_{\mathrm{m}} \\
(\mathrm{mm} / \mu \mathrm{s})\end{array}$ & $\begin{array}{c}\text { Angle } \\
\gamma(\mathrm{deg})\end{array}$ & $\begin{array}{c}\mathrm{P}_{\mathrm{e}} \\
(\mathrm{GPa})\end{array}$ & $\begin{array}{c}\mathrm{P}_{\mathrm{m}} \\
(\mathrm{GPa})\end{array}$ \\
\hline 0 & 0 & 1.13 & 0.0 & 37.3 & 56.5 \\
30 & 30 & 1.00 & 3.3 & 35.6 & 48.5 \\
45 & 45 & 0.87 & 4.0 & 33.9 & 40.9 \\
60 & 60 & 0.74 & 4.2 & 31.4 & 33.3 \\
75 & 75 & 0.60 & 3.8 & 26.7 & 26.0 \\
90 & 75 & 0.61 & 4.0 & 26.3 & 26.3
\end{tabular}

The results of the simple theory are plotted in Fig. 5-4 along with the code results and the few pieces of oblique angle data. The $\mathrm{Y}$-axis is $\mathrm{u}_{\mathrm{m}} / \mathrm{u}_{\mathrm{m}}\left(0^{0}\right)$, so that the straight-ahead data is taken as the reference. Bigplate is unique is that it furnishes its own $0^{\circ}$ reference, then goes on the deliver various oblique angles in the same experiment. At $90^{\circ}$, pure transverse data comes from our cylinder and sideways plate push experiments; these were described in Chapter 3 . We note that no correction is made for the cylinder wall thickness so that the ratio could be low by several percent. Both copper and tantalum plate data is included. In between are several aluminum wedge points from a paper of 35 years ago. 3 The authors had no interest in oblique shocks as such but were designing a method of getting an entire metal Hugoniot in one shot. There is no way to correct their jump-offs and we can but take the data as listed. The small amount of pertinent data is surprising after so many years of explosives research.

\section{Detailed Code Studies}

The problem was modeled using HEMP. Throughout, five zones of metal were used with a zone size of $0.33 \mathrm{~mm}$ (along the metal wall) by $0.10 \mathrm{~mm}$ (30 zones $/ \mathrm{mm}^{2}$ ). A type- 1 slideline was placed between the explosive and the metal. The Steinberg-Guinan material model was used for the copper. The combination beta/program burn was used with point detonation. The cylinder JWL ( $A=$ 
7.65) and the 381 JWL (Table 3-1) were used along wth the CO Look-Up Table (Table 3-2). It is recalled that the cylinder JWL is essentially the 381 , so that no appreciable difference is expected. The program was written to show the velocity and distance along the lines of sight of the five Fabry beams.

Fig. 5-5 shows the results of running $25 \mathrm{~mm}$-radius copper plates with uniform explosive meshes and the $A=7.65$ JWL for LX-14. All variations showed the proper decrease of the jump-off velocity with the distance off-axis, but the absolute height varied considerably. A mesh of 4 zones $/ \mathrm{mm}^{2}$ was too coarse and the jump-off velocity was very low. A mesh of $17 \mathrm{zones} / \mathrm{mm}^{2}$ was adequate as long as the aspect ratio remained between 1 and 2.5 , where the ratio is the side length along the wall divided by the side length perpendicular. An aspect ratio of 0.4 gave low results. A mesh of 64 zones $/ \mathrm{mm}^{2}$ was good, even for the 0.4 aspect ratio, although the aspect ratio of 1.0 was better and 2.5 better yet. The best results are obtained for skinny zones lying with the long axis parallel to the metal wall.

The dynamics of the zoning was studied by setting both the copper and the explosive with uniform meshes of aspect ratio 1.00 . The results in the explosive at $10 \mu$ s are shown here, with the $90 \mathrm{~mm}$ values representing the unchanged mesh.

\begin{tabular}{ccc}
$\begin{array}{c}\text { radius } \\
(\mathrm{mm})\end{array}$ & $\begin{array}{c}\text { aspect } \\
\text { ratio }\end{array}$ & $\begin{array}{c}\text { zones/ } \\
\mathrm{mm}^{2}\end{array}$ \\
\hline 90 & 1.00 & 25 \\
70 & 0.67 & 17 \\
50 & 0.47 & 11 \\
30 & 0.29 & 11 \\
0 & 0.28 & 8
\end{tabular}

The explosive are pulled out in the forward direction because of the mass flow. As one moves near the axis where the problem has run longer, the aspect ratio and the zones/mm $\mathrm{m}^{2}$ both decline. The copper, however, is compressed so that the aspect ratio rises from 1.0 at $90 \mathrm{~mm}$ to 1.8 at $0 \mathrm{~mm}$. The density also rises from 100 to 128 zones $/ \mathrm{mm}^{2}$. A separate run showed that square zones produced a better jump-off in copper than did higher density zoning with a large aspect ratio.

Another problem appeared in an early HEMP run. The mesh contained two different zone sizes: $0.33 \times 0.30 \mathrm{~mm}(10 \mathrm{zones} / \mathrm{mm})$ in the rear of the explosive and $0.33 \times 0.14 \mathrm{~mm}(22 \mathrm{zones} / \mathrm{mm})$ in the $33 \mathrm{~mm}$ nearest the plate. As seen in Fig. 5-6, the jump-off velocities were constant from 0 to 20 $\mathrm{mm}$ distance off-axis; then they decreased as expected. It was caused by refraction at the mesh 
density interface. The flow was directed forward and broke up the jet that will be described below. The extra forward flow pushed the center $40 \mathrm{~mm}$ diameter of the plate evenly, an effect not seen in the experiment. This same effect was seen by Craig Tarver in a 36,000 zone DYNA2D run in which the explosive, although uniformly zoned, had an aspect ratio of about 0.4 , i.e. the zones were skinny perpendicular to the plate. Moreover, no slideline was present and the two materials were merged. In Bigplate modeling, this center-push problem is to be looked for and removed.

\section{Angular Behavior in the Code}

We return to see what the code predicts regarding directions of motion. The various rays and their angles are illustrated in Fig. 5-2. An actual snapshot of Bigplate alligned in the geometry of Fig. 5-2 is shown in Fig. 5-7, where 64 zones $/ \mathrm{mm}^{2}$ have been used. The explosive is at the left and the copper at the right. The pressure contours show where the wave fronts are: the detonation front is at the top center, the reflected wave at the bottom center, and the refracted wave at the bottom right. The angles are all defined as being between the rays and the normals to the initial wall position. This is the same as the wave front with the initial wall position.

The point of impact with the inner wall at $2.5 \mu \mathrm{s}$ in Fig. 5-7 is at the intersection of the wave fronts at about $1.01 \mathrm{~cm}$. The angle of incidence, $\alpha$, is about $30^{\circ}$. We have not defined the angle of refraction, $\beta$, which, from Snells' Law , is given by:

$$
\frac{\mathrm{D}}{\sin \alpha}=\frac{\mathrm{U}_{\mathrm{m}}}{\sin \beta}
$$

where $U_{m}$ is the wave velocity in the copper.

The vectors in Fig. 5-7 are the nodal vectors, which are indicators of the mass flow. The scale has been set equal in the X-and Y-directions so that the direction of these vectors can be better seen. We see that the vectors are perpendicular to the detonation front, as expected. However, right at the inner metal surface, the vectors are suddenly bent upward. Here we see different directions for the mass flow and the wave velocity. This same general direction also occurs in the product gas at the bottom center, which is being injected up along the inner wall. Inside the copper, the vectors have been turned almost perpendicular. By the time the wave reaches the outside wall, its velocity is completely perpendicular to the surface. 
A summary of the code angles at the instant of detonation is shown in Fig. 5-8. LX-14/copper Bigplate is used up to $90^{\circ}$, where the cylinder is put in. The X-axis is the geometric value of $\alpha$ or $\beta$. The code's $\alpha$, when against geometric $\alpha$, is very close up to $75^{\circ}$. At this point, the code's wave front sticks at $75^{\circ}$ as do measured cylinder fronts. For $75^{\circ}<\alpha<90^{\circ}$, $\alpha$ is just a geometric quantity. The angles of refraction also fit when plotted against geometric $\beta$. It may seem surprising that Snell's Law fits so well. The angles of the nodal vectors taken from the explosive 2 or more zones away from the wall also fit geometric $\alpha$. What does not fit are the nodel vectors in the zone next to the wall. These are turned sharply upward in Fig. 5-7 and cause the extreme deviation seen in Fig. 5-8.

The appearance of the nodal vectors in Fig. 5-7 is that product gas is being jetted upward from the center of the plate, where the impact has already taken place. All along this region, the vectors are turned upward to large values. We have noted in Appendix B that a shock wave is about $0.05 \mu \mathrm{s}$ long. The wave velocity moving upward along the wall is about $20 \mathrm{~mm} / \mu \mathrm{s}$ at $10 \mathrm{~mm}$ off-axis. This produces a region of about $1 \mathrm{~mm}$, which is the space sized out in the figure, and which shows a jet coming in behind the reflected wave. The presence of the jet has led to the idea that the explosive flow is "transverse" for $\alpha \approx 30^{\circ}$, i.e. that this case and the cylinder are the same. This seems reasonable as far as the appearance of Fig. 5-7 goes. However, it cannot be true, because the particle velocity of the copper plate is $1 \mathrm{~mm} / \mu \mathrm{s}$ at $30^{\circ}$ but only $0.5 \mathrm{~mm} / \mu \mathrm{s}$ for the cylinder. So they are not the same. This may be checked by looking at the corresponding cylinder picture in Fig. 4-4. There we do not see any reflected wave or the "jet" next to the inner surface.

More detailed behavior is shown in Fig. 5-9, which plots the derivitives of the forward and radial positions during the $0.05 \mu \mathrm{s}$ instant of impact. Inside the explosive 2 or more zones, the radial flow is large, representing the jet at work. But in the nearest zone, next to the slide line, the radial flow is greatly reduced and most of the motion is forward. This is the beginning of the conversion from the angular to the perpendicular flow, which continues in the metal plates. Physically, the pressure moves to where there is the most give, which is the bending metal plate. The angle defined by these derivitives in the second zone is about $51^{\circ}$, in the nearest zone, it is only $16^{\circ}$ so that the average is close to $\alpha \approx 30^{\circ}$. Thus, the nodal vectors do not really show the detailed directional behavior as well as they should. For this reason, we have used $\alpha \approx \Theta$ in the simple calculation.

In the code for the cylinder, the detonation velocity is at $\alpha=90^{\circ}$ but the nodal vector at the surface is at $75^{\circ}$. We recall that the measured angle of incidence of the detonation front is $72^{\circ}$ to $74^{\circ}$ for both HMX and TATB. 4 For HMX, the detonation front lags with a backward blip which, of corse, does not appear in the code. This suggests that $D$ and $u_{p}$ are always parallel but diverge in our current code in the region next to a wall. 


\section{EOS Comparison in the Code}

The mesh that was finally chosen was uniform with $5 \times 400$ zones in the copper (40 zones $/ \mathrm{mm}^{2}$, aspect ratio 1.0 ) and $100 \times 200$ in the explosive (10 zones $/ \mathrm{mm}^{2}$, aspect ratio 2.5 ). The uniform mesh was closen to avoid the refraction effects described above. Zone tangling occured at 17 $\mu \mathrm{s}$ and Steve Anderson fixed this by removing the outermost five zones at $5 \mu \mathrm{s}$.

Figs. 5-10 and 5-11 show the \% comparison at the 0 and $20 \mathrm{~mm}$ radial positions of the Fabry velocity with the $A=7.65$ cylinder JWL and the CO Look-Up Table. The JWL is good at $0 \mathrm{~mm}$ and fair at $20 \mathrm{~mm}$. The Table is not so good at both positions.

\section{Bigplate as an EOS Test}

Could some version of Bigplate be used to replace the cylinder test for EOS? It appears equally as sensitive at long times. The $0.5 \mathrm{~mm}$ copper plate is larger than the reaction zone of the HMX so that the Bigplate configuration is an effective EOS measurement geometry for this ideal explosive. HEMP runs showed that spall effects began with a $1 \mathrm{~mm}$ copper plate and rapidly increased with thickness. The effect of spall is to prolong the first plateau at a lower level with a lower velocity at long times and also to destroy any hope of getting an EOS. The effects of spall are greatest in the center of the plate where pressures are highest. The 1.3 to $2.5 \mathrm{~mm}$ cylinder walls are thicker and will screen out the reaction zones effects of less ideal explosives. Also, the pressures are lower so that spall is likely to occur. Bigplate also lacks the other big cylinder advantage: that the geometric volume is close to the actual explosive volume, because of the confinement. A final advantage to the cylinder is that the approach to steady state is indefinite, so that any slowness in the build-up of the burn model is easy to fix by adding more length. Bigplate, however, has a fixed length and the burn turn-on slowness could be a problem, expecially if the problem were lightly zoned.

Bigplate, however, has already focused attention on the angular behavior of shock waves and on how good the EOS shows jump-off. Jump-off is not important in the cylinder test even though it starts at $\mathrm{v} \approx 0.85$ and is perfectly capable of saying something about small volume behavior. One reason is that the cylinder test has been defined for $2<v<7$, where the wiggles have gone away and the geometric volume is well-defined. Another reason is that it takes at least $12 \mu$ s to reach a steady state jump-off as opposed to $2.2 \mu \mathrm{s}$ in Bigplate. The need for long code runs has discouraged consideration of cylinder jump-offs. 
Bigplate's contribution is to highlight the effects of an oblique plate collision, all in one shot. This may eventually give it the status of a true EOS test.

\section{References}

1. The oblique shock wave hitting a plate is different from the usual "oblique shock wave" problem in gasdynamics. Here, a small wall angle is placed is the way of a supersonic fluid flow and a shock wave results at this point. The same gas flow is involved so that sideways translation and mass continuity are required. A tangent formula results. See A. H. Shapiro, The Dynamics and Thermodynamics of Compressible Fluid Flow (Ronald Press, New York, 1953), I, pp. 532, 535.

2. W. E. Deal, J. Chem. Phys. 27, 796 (1957).

3. S. Katz, D. G. Doran and D. R. Curran, J. Appl. Phys. $\underline{30} 568$ (1959).

4. P. C. Souers and L. C. Haselman, Jr., Detonation Equation of State at LLNL, 1993, LLNL Report UCRL-ID-116113 (March, 1994), p. 7-2. The angle there is offset $90^{\circ}$ from the angle defined here. 


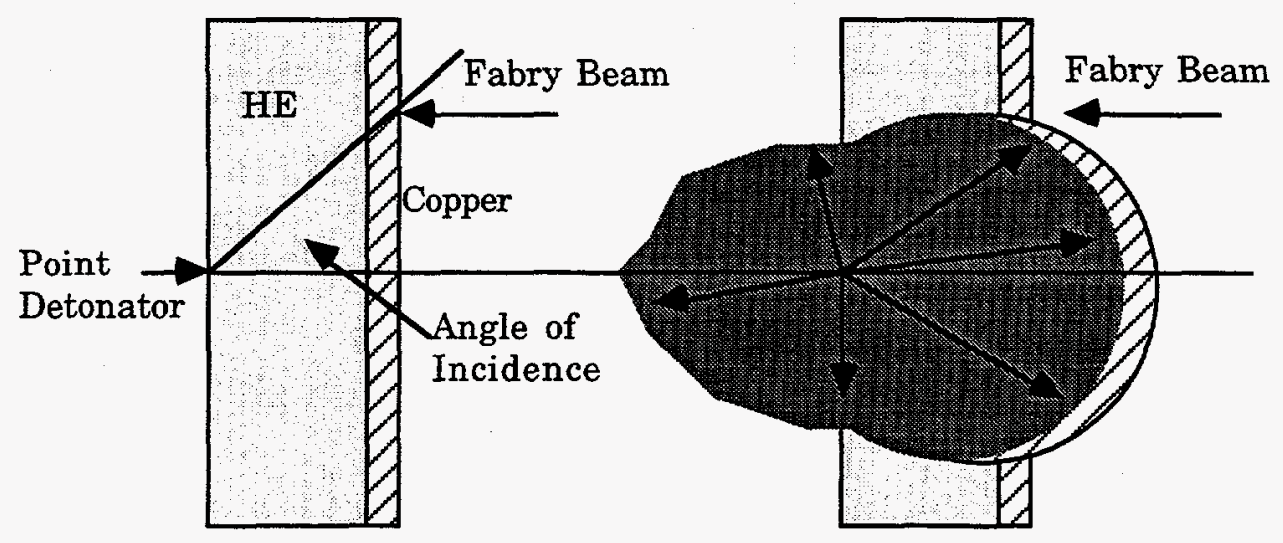

Fig. 5-1. Schematic of the Bigplate experiment- an explosive disk and copper plate. The spherical point detonator sits in the center of the back of the explosive and makes it a 2-D geometry.

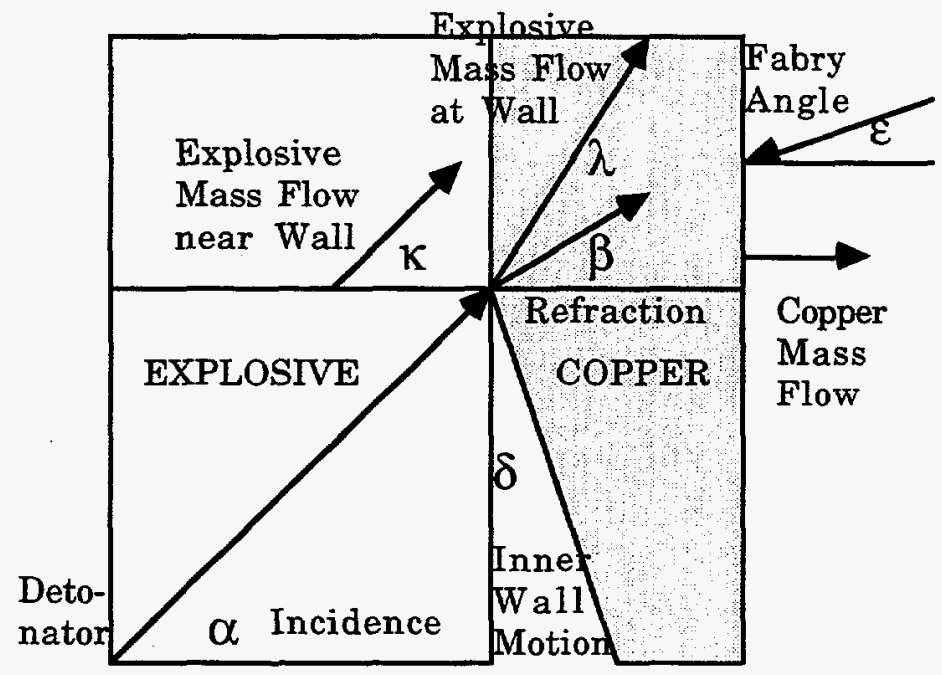

Fig. 5-2. Angles and rays in the description of Bigplate. A sideview is shown. 


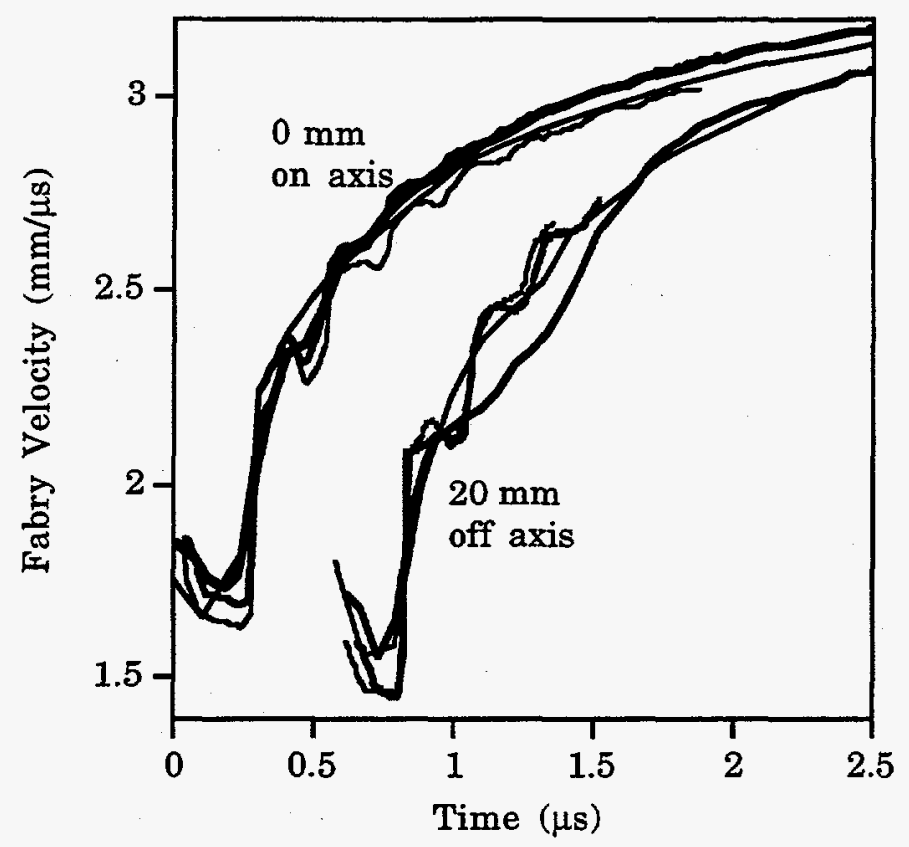

Fig. 5-3. Fabry data from all three samples to see if the $0.02 \mathrm{~g} / \mathrm{cc}$ density difference can be seen onaxis and $20 \mathrm{~mm}$ off-axis. Despite hints at $0 \mathrm{~mm}$, we conclude there is no significant difference. Run $891 D 2$ is the heavy line; 891D3 is the light line.

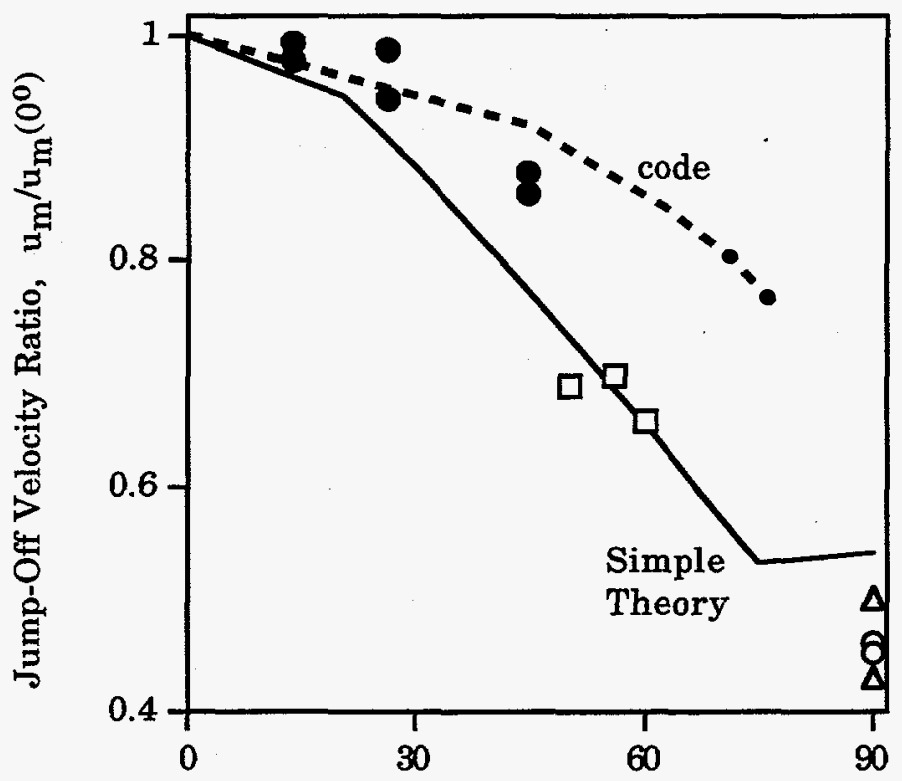

Geometric Angle of Incidence, $\alpha$ (degrees)

Fig. 5-4. Metal plate jump-off velocities as a function of the geometric angle of incidence for shock waves hitting at oblique angles. Bigplate data is at the top; aluminum data in the center and cylinders at the bottom right. 


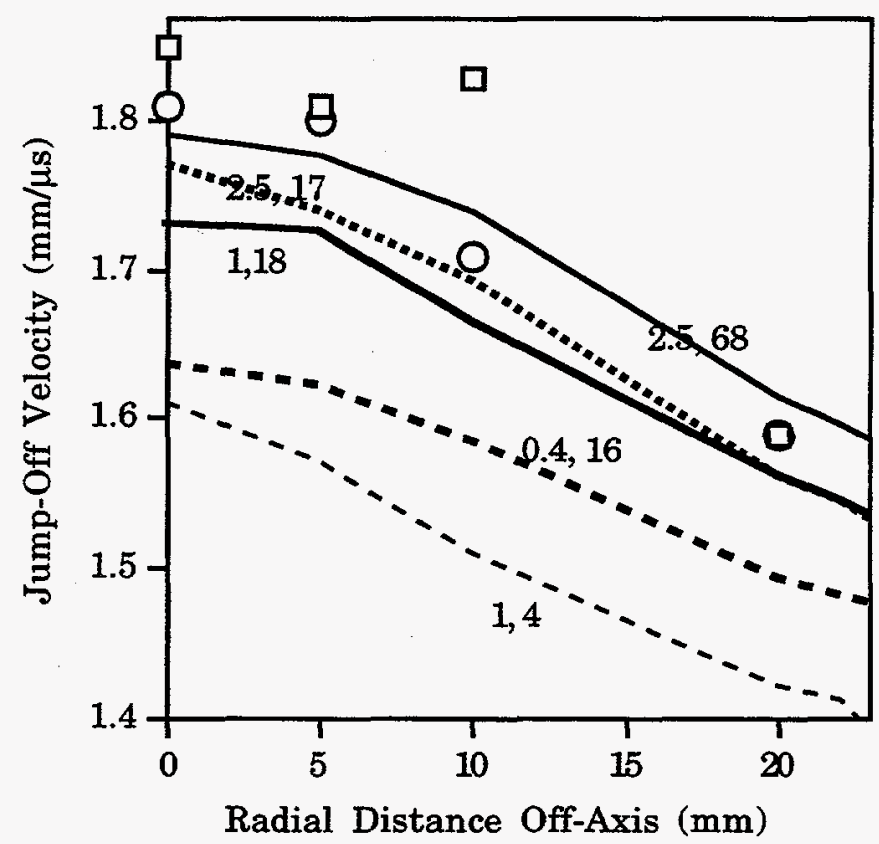

Fig. 5-5. Jump-off velocities in HEMP using a uniformly zoned explosive grid of the listed aspect ratio and number of zones per $\mathrm{mm}^{2}$. The Bigplate data are the circles and squares. The use of 17 zones $/ \mathrm{mm} 2$ is adequate if the aspect raio is 1 or more. Good results are obtained for all aspect ratios with a density of 64 zones $/ \mathrm{mm}^{2}$ although 2.5 is best.

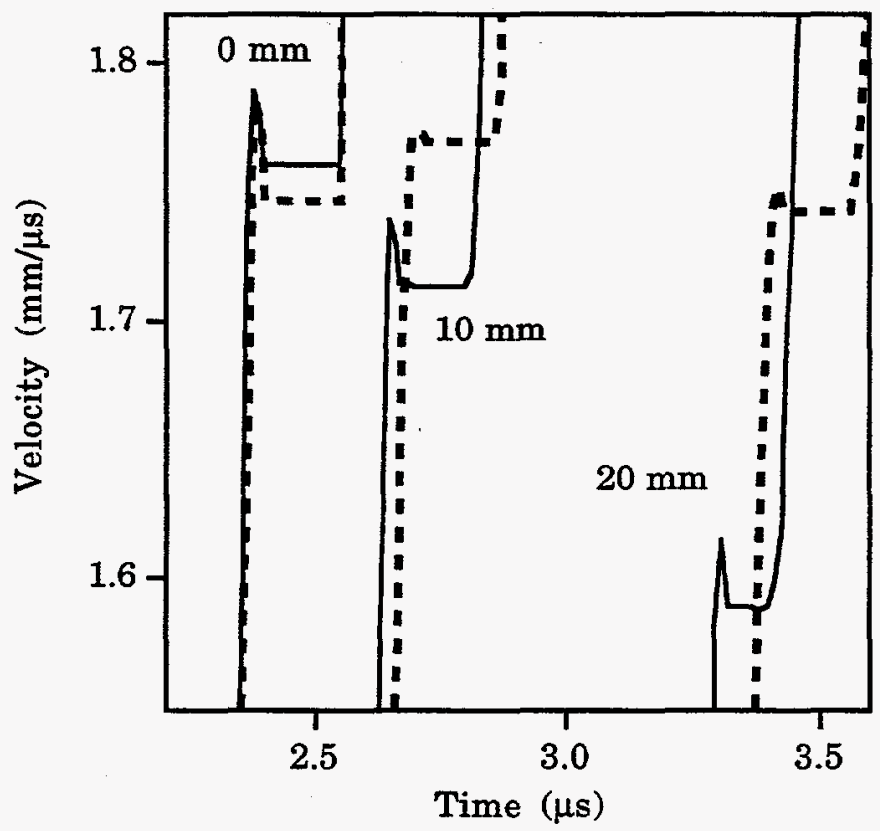

Fig. 5-6. Jump-off behavior in HEMP for an uniformly-sized mesh (full lines) and a mesh with two different explosive zone sizes (dashed). The correct behavior is the decreasing jump-off with off-axis distance, which is listed. 


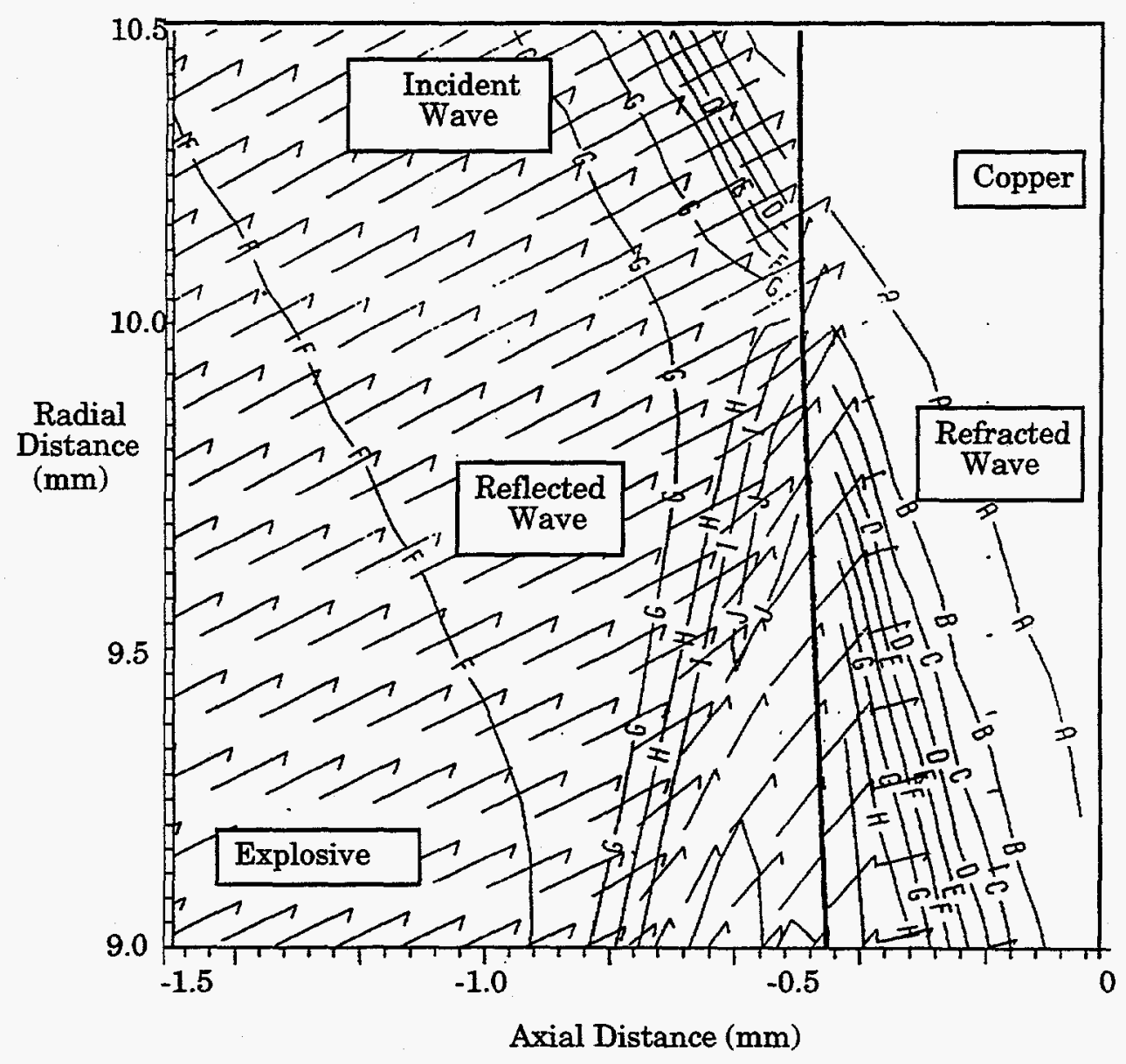

Fig. 5-7. Bigplate view for an angle of incidence of $30^{\circ}$. The explosive is at the left the copper wall at the right. The waves are shown by the pressure contours. The detonation wave is at the center top; the reflected wave is at the center bottom; the refracted wave in the copper is at the lower right. The arrows are the nodel vectors and they are indicative of the mass flow. The $1 \mathrm{~mm}$ length along the wall is about the space covered in a real shock wave "instant of impact". 


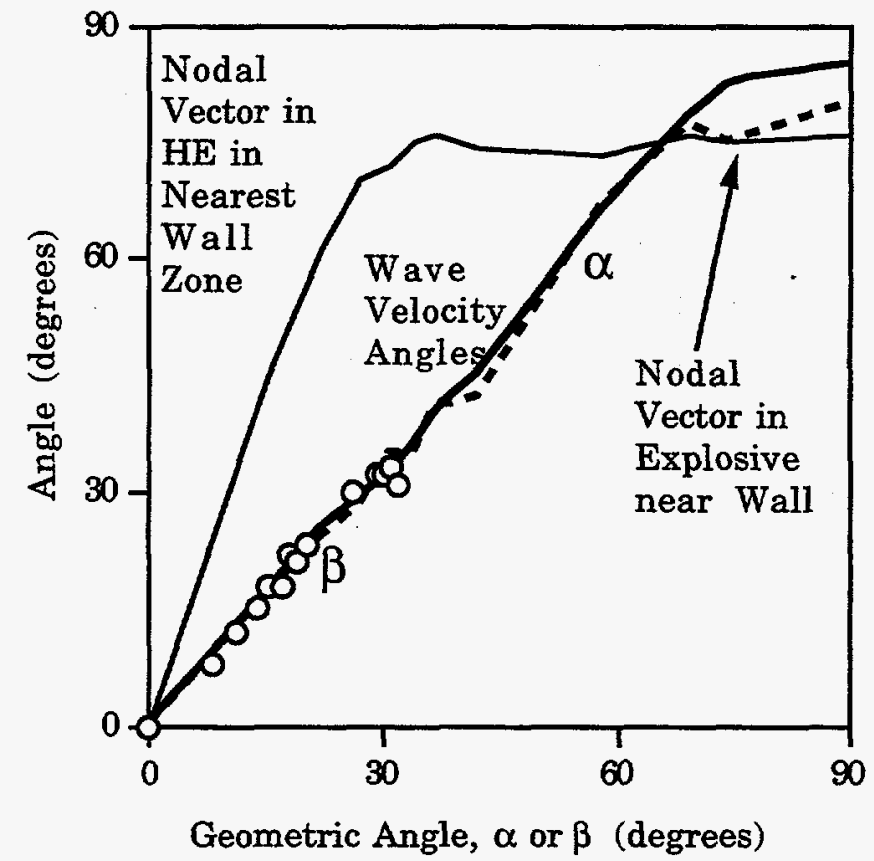

Fig. 5-8. Comparison of the geometric angles with the calculated ones in the HEMP code for Bigplate. All are close except for the mass vector in the explosive zone nearest the wall and the behavior for angles greater than $75^{\circ}$. The cylinder is used for the $90^{\circ}$ point. Note the peak at $30^{\circ}$ in the nodal vector of the explosive nearest the wall.

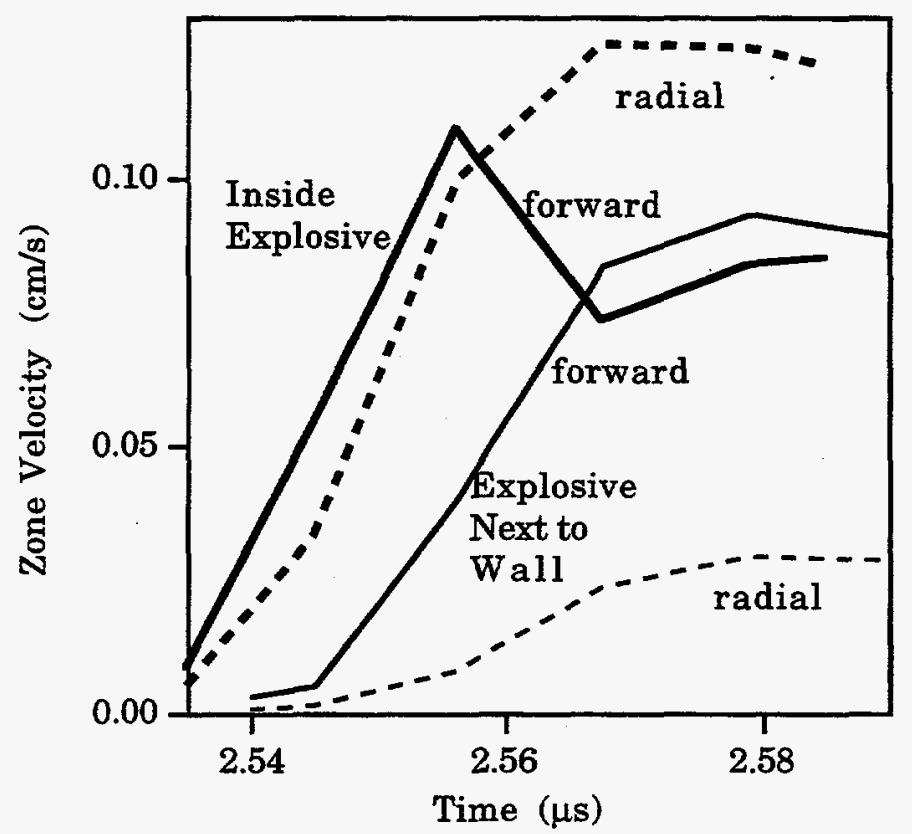

Fig. 5-9. Derivitives of position in the HEMP zones inside the explosive and next to wall as measured for $a \approx 30^{\circ}$. Inside the explosive, the radial flow is considerable. At the wall, it gets jammed forward. 


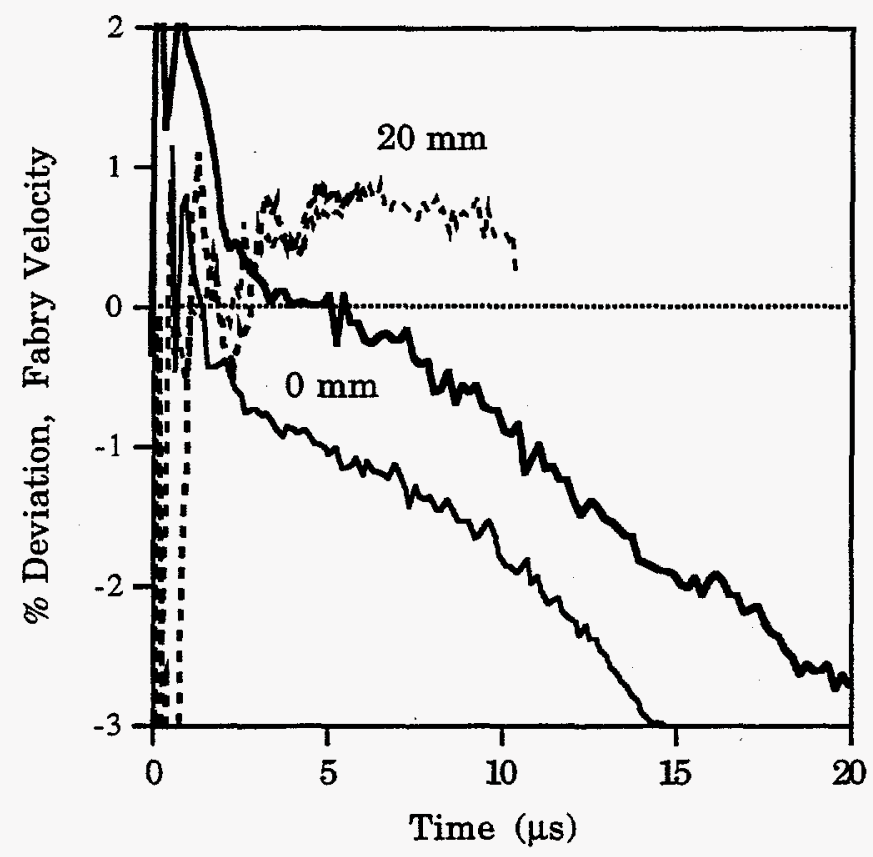

Fig. 5-10. Percent comparison of the LX-14 A = 7.65 cylinder JWL with the data from runs 891D1\&2 and $891 \mathrm{D} 3$ at the radial positions of 0 and $20 \mathrm{~mm}$. The $20 \mathrm{~mm}$ agreement is good; the $0 \mathrm{~mm}$ fair.

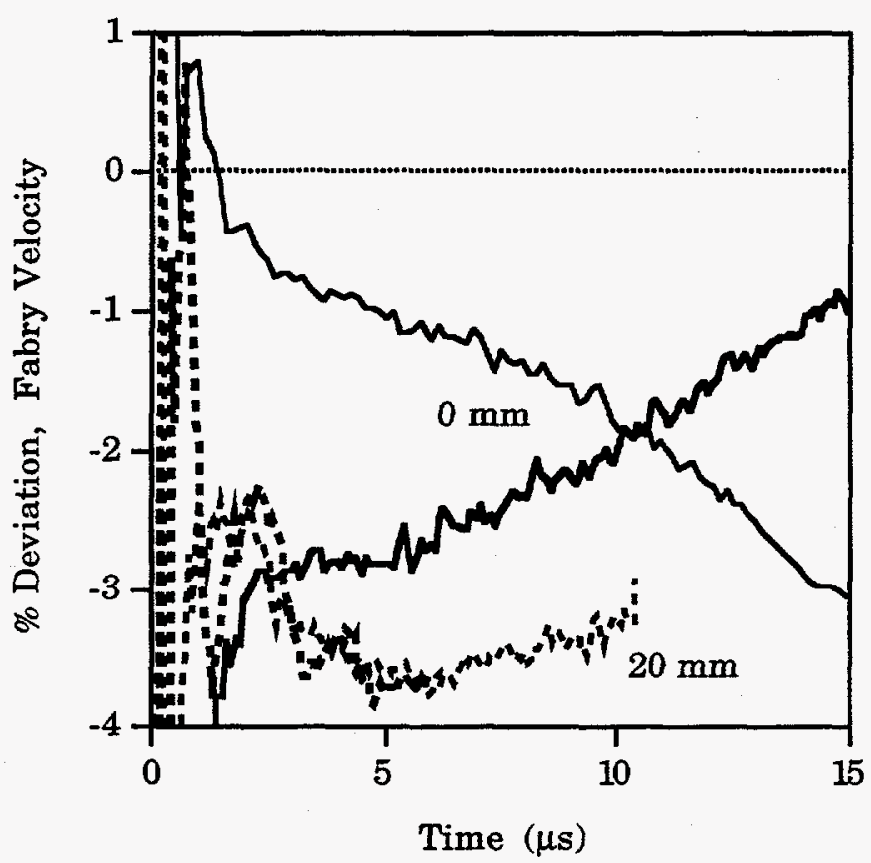

Fig. 5-11. Percent comparison of the LX-14 CO Table with the data from runs 891D1\&2and 891D3. The agreement iis poor at both positions. 


\section{Chapter 6. Plates and the Expanding Sphere}

\section{Electric Gun Flatness}

About 20 years ago, mutiple calibration runs of the electric gun flyer (slapper detonator) were made. ${ }^{1}$ A schematic of the apparatus is shown in Fig. 6-1. A capacitor bank was discharged through an aluminum foil, which vaporized and pushed a thin kapton (plastic) flyer up a gun barrel into a flasher block. The block usually had a $1 \mathrm{~mm}$ notch for velocity calibration. The streak camera looked down from above through the flasher block and was swept at $20 \mathrm{~mm} / \mu \mathrm{s}$ parallel with the direction of the current in the aluminum. The picture showed a bright line as the flyer compressed the air in front of the flasher block. Upon impact, the light was quenched and the picture turned black. The edge allowed a good measurement of the impact with a listed $10 \mathrm{~ns}$ time resolution. A ruler was photgraphed in the picture, thus allowing conversion to the real distance. The runs were made to calibrate the flyer velocity, which appeared to decrease as the capacitor bank aged, then rose again upon capacitor refitting. We are here more interested in considering the flatness of the shots. This is obtained by measuring the oscillations, converting them to real distance and dividing by the sweep speed of $20 \mathrm{~mm} / \mu \mathrm{s}$.

We shall divide the flyer front into three zones: the inner half, the intermediate quarter and the outer quarter near the edge. The quality of the front is defined as follows:

\begin{tabular}{llll}
\multicolumn{1}{c}{ Zone } & \multicolumn{1}{c}{ Good (1) } & \multicolumn{1}{c}{ Average (2) } & \multicolumn{1}{c}{ Bad (3) } \\
\hline Center half & $\begin{array}{l}\text { flatness to } \pm 5 \mathrm{~ns} ; \text { looks } \\
\text { smooth at } 20 \mathrm{~mm} / \mu \mathrm{s}\end{array}$ & $\begin{array}{l}\text { same, or with tilt of } \\
10 \mathrm{~ns} \text { from the center. }\end{array}$ & $\begin{array}{l}\text { Big knify oscilla- } \\
\text { tions of tens of ns }\end{array}$ \\
$\begin{array}{llll}\text { Intermediate } \\
\text { quarter }\end{array}$ & $\begin{array}{l}\text { oscillations of } \pm 10 \text { to } \\
15 \mathrm{~ns} ; \text { looks bumpy. }\end{array}$ & $\begin{array}{l}\text { same, or with tilt of } \\
15 \text { to } 20 \mathrm{~ns} \text { from center }\end{array}$ & $\begin{array}{l}\text { Bad tilt; big knify } \\
\text { oscillations. }\end{array}$ \\
Edge quarter & Flat on both & $\begin{array}{l}100 \mathrm{~ns} \text { lag in outer } \\
\text { half } \\
\text { near the edge }\end{array}$ & $\begin{array}{l}\text { Lag is hundreds of ns } \\
\text { of one side. }\end{array}$
\end{tabular}

Fig. 6-2 shows an example of a good and an average slapper front. Both are acceptable for plate shots. 
Many of the shots were done by Dick Weingart and C. Honodel. From the raw data files of Ron Lee, we find these results, all for 0.25 to $0.30 \mathrm{~mm}$ kapton flyers in lucite gun barrels:

\begin{tabular}{|c|c|c|c|c|c|c|c|c|c|}
\hline \multirow{3}{*}{$\begin{array}{l}\text { Dia. } \\
\text { (mm) }\end{array}$} & \multirow{3}{*}{$\begin{array}{c}\text { Barrel } \\
\text { Length } \\
\text { (mm) }\end{array}$} & \multirow{3}{*}{$\begin{array}{l}\text { Volts } \\
(\mathrm{kV})\end{array}$} & \multirow{3}{*}{$\begin{array}{l}\text { No. } \\
\text { Shots }\end{array}$} & \multirow{2}{*}{\multicolumn{4}{|c|}{ \% Good\& }} & \multirow{3}{*}{$\begin{array}{c}\% \text { Ave. } \\
\text { Edge }\end{array}$} & \multirow{3}{*}{$\begin{array}{l}\text { Where } \\
\text { made }\end{array}$} \\
\hline & & & & & & & & & \\
\hline & & & & Center & Int. & Center & Int. & & \\
\hline 25 & 1.3 & 40 & 4 & 100 & 100 & 100 & 100 & 100 & LLNL \\
\hline 38 & various & 25 to 40 & 8 & 88 & 75 & 100 & 88 & 38 & Bendix \\
\hline 25 & 5.0 & 40 & 63 & 25 & 19 & 79 & 57 & 56 & LLNL \\
\hline 25 & 5.0 & 40 & 48 & 38 & 25 & 75 & 73 & 73 & Bendix \\
\hline 25 & 5.0 & 40 & 9 & 22 & 22 & 67 & 67 & 67 & ELNL \\
\hline 51 & 5.6 & 40 & 10 & 20 & 20 & 60 & 60 & 40 & LLNL \\
\hline 51 & 5.6 & 30 to 40 & 10 & 0 & 0 & 70 & 70 & 60 & Bendix \\
\hline
\end{tabular}

Samples with zero or one measurement notch were used above. Multi-notch shots were too hard to read. Potentials of $25 \mathrm{kV}$ usually produced full velocity although $40 \mathrm{kV}$ soon became the standard. Going to a wider flyer by itself does not ensure flatness. Perhaps the optimum ratio of barrel length to diameter is the quantity that needs to be optimized. The tightness of fit of the barrel to the flyer is given as another intangible. Flyers made in production at Bendix (now Allied-Signal, Kansas City) were no better than the LLNL homemade. Out of ten thousand flyers made over a decade, the quality was just as good at the beginning as at the end. Another small set of 2 to 3 each appeared to say that lucite guns were slightly better than pure metal or metal-lucite composities. The reason for the high incidence of good fronts in the $38 \mathrm{~mm}$ flyers is unknown. We can say from the $6325 \mathrm{~mm}$ shots is that flyer velocity per itself is not the problem. These flyer velocity results were found:

\begin{tabular}{lccc} 
& Good & Intermed. & Poor \\
\cline { 2 - 4 } Average $(\mathrm{mm} / \mu \mathrm{s})$ & 5.23 & 5.31 & 5.45 \\
\% Stdev & 4 & 3 & 5
\end{tabular}

Excluded from the poor list are the bottom 4 cases, where the velocities really are too low. These constitute $6 \%$ of the total. The remaining $94 \%$ shown above all have the same average flyer velocity, with the deviation caused by capacitor bank changes. The reason for poor quality shots appears to be faulty allignment because poor shots are invariably badly tilted. The reason for the oscillations in average shots is unknown. 
We can make two comments about electric gun plate data. First, the 1-D time is not set by the distance to the edge but to about $3 / 4$ of that distance, because of the effects near the edge of the flyer. For a $51 \mathrm{~mm}$ sample, this is about $2 \mu \mathrm{s}$. For a $25 \mathrm{~mm}$ flyer, the $1 \mu$ s time may be too little to get adequate data. The second point is that the overall result is 2 good-plus-average runs out of 3 , which is close to what is seen: one failure in 3 shots. Most of the old data was limited to about 3- 4 shots for each set of dimensions and this is not enough. A set of at least 6 to 8 samples of identical dimensions is needed to get good numbers.

Another variable is the velocity of the flyer. As shown below for kapton, this increases with the increase in the lucite barrel length (with the unexplained exception of the $51 \mathrm{~mm}$ shots).

Presently, a barrel length of $5.6 \mathrm{~mm}$ ( 0.22 inches) is used as the standard. Ron Lee believes that the tendency for the flyer to turn sideways increases with the length of the barrel and suggests that 1.5 $\mathrm{mm}$ might be the best selection for quality. However, the use of a reproduceable way to clamp the assembly together may be the most important factor.

\begin{tabular}{|c|c|c|c|c|c|c|}
\hline \multirow{2}{*}{$\begin{array}{l}\text { Diam. } \\
(\mathrm{mm})\end{array}$} & \multirow{2}{*}{$\begin{array}{l}\text { No. } \\
\text { Shot }\end{array}$} & \multirow{2}{*}{$\begin{array}{c}\text { Flyer } \\
\text { Velocity } \\
(\mathrm{mm} / \mathrm{ss})\end{array}$} & \multicolumn{3}{|c|}{$\begin{array}{c}\text { Flatness } \\
\text { of Flyer Front }\end{array}$} & \multirow{2}{*}{$\begin{array}{l}\text { Barrel } \\
\text { length } \\
(\mathrm{mm})\end{array}$} \\
\hline & & & Cen & Int & Edge & \\
\hline 38 & 1 & 3.5 & 1 & 1 & 3 & 1.3 \\
\hline 38 & 1 & 3.5 & 2 & 2 & 3 & 1.3 \\
\hline 25 & 4 & 3.8 & 2 & 2 & 3 & 1.3 \\
\hline 38 & 1 & 4.1 & 1 & 1 & 2 & 3.2 \\
\hline 25 & 29 & 5.2 & 2 & 2 & 2 & 5.0 \\
\hline 38 & 1 & 4.8 & 1 & 1 & 2 & 5.1 \\
\hline 25 & 8 & 5.7 & 2 & 2 & 2 & 5.6 \\
\hline 51 & 9 & 3.0 & 2 & 2 & 2 & 5.6 \\
\hline 38 & 1 & 5.2 & 1 & 3 & 3 & 12.2 \\
\hline
\end{tabular}

\section{Small Metal Plate Shots and the Reaction Zone}

Fig. 6-3 shows the classic way of determining the width of the explosive reaction zone from 1D metal plate shots. The unconfined-face jump-off velocity (or the impedance-determined detonation pressure) is plotted as a function of the metal thickness. The thin-pulse explosive spike degrades quickly in the metal, if the reaction zone is small, as is true for LX-14. It degrades more slowly for the LX-17, which has a reaction of several $\mathrm{mm}$, so that it might be difficult to distinquish a change of slope. The data of Fig. 6-1 suggests that the reaction zone for LX-14 is about $0.2 \mathrm{~mm}$ and that for 
LX-17 is greater than $2.5 \mathrm{~mm}$. For this method to really work, enough thicknesses of explosive and plate have to be run to be sure the reaction zone region is found. This is rarely done.

What can we learn if all we have is a handful of electric gun shots? We can use the $R_{1}=4.5$ JWL as the "perfect cylinder"-no-reaction-zone "standard." If we use this, we quickly find that many electric gun velocities are higher than expected. This is the result of seeing the reaction-zone through the thin metal plate. The experimental electric gun shot data is assembled in Table 6-1. An example of the effect is shown in Fig. 6-4 for $1.767 \mathrm{~g} / \mathrm{cc}$ PETN. The explosive is $10 \mathrm{~mm}$ thick and was driven at $4.1 \mathrm{~mm} / \mu \mathrm{s}(19 \mathrm{GPa}$ ). The tantalum plate was $0.10 \mathrm{~mm}$ (4 mils) thick with a $0.025 \mathrm{~mm}$ (1 mil) silicone glue joint between it and the explosive. Fabry-Perot interferometry was used to measure the plate velocity.

All this, including the glue joint, was modeled with the LLNL 1-D code. As seen in Fig. 6-4, the data ( $\mathrm{A}$, heavy line) is way above that predicted by the cylinder JWL with $A=6.179$ (C, solid) from the previous chapter. This curve includes the energy of the flyer. If this energy is removed, the curve ( $\mathrm{B}$, dotted) is only slightly lower, so that flyer velocity is not an issue. Another approach is to wonder if additional energy added slightly after the $R_{1}$ term could be present. We, therefore, tried the 3-exponential JWL form

$$
P_{S}=A \exp (-4.5 v)+\operatorname{Eexp}(-3.0 v)+B \exp (-1.5 v)+C / v(1+\omega)
$$

Curve $E$ (dotted) is such a 4-term JWL. There is no way to fit the plate data and still maintain the expected cylinder energies at the volumes of $2.2,4.1$ and 6.5. Curve $D$ (dashed) is a force fit with $A=$ 5.997, $R_{1}=4.5$ and $R_{2}=1.2$. The detailed $J W L$ is listed in Table 6-2. Although we have fit the data, the extrapolated cylinder energies are $20 \%$ too high! This means we have shifted the same energy $\mathrm{E}_{0}$ so we get the plates to move faster. If this behavior continued, the cylinders would be pushed too fast.

Because we cannot use a JWL to describe ZND behavior, and because we have no quantitative data at this stage, we instead use this procedure. We 1) leave $\omega$ and $\mathrm{E}_{0}(\infty)$ the same, 2) decrease $R_{2}$ toward 1.0 changing $\Gamma_{j}+1$ only slightly if necessary, and 3 ) raise $R_{1}$ with $R_{2}=1.0$ as a last resort. We end up with a JWL that looks similiar to what we had, but the energies at the cylinder volumes of $2.2,4.1$ and 6.5 are all higher. We are rearranging the early-time push to match the plate velocity, but the energy, if it continues, would overdrive the cylinder. The \% more of apparent cylinder energy is a qualitative measure of the presence of the reaction zone. One thing that does not change much in this process is the detonation pressure, which rises slightly as $\mathrm{R}_{2}$ 
decreases. We cannot physically add in the spike pressure is a satisfactory way, so we have simply reduced the number of variables.

Another problem of the electric gun is that many powerful explosives are always underdriven. A $40 \mathrm{keV}$ shot produces about $30 \mathrm{GPa}$ from impedance calculations and this lies below the detonation pressure of many explosives. We assume that the time to rise to full power is quick.

All the percent deviations in energy are plotted in Fig. 6-5. The surprises must be taken in concert with the data in Table 6-1. Ron Lee noted that XTX-8003 (80\% PETN) has a critical radius of $0.1 \mathrm{~mm}$ whereas that for PBX-9501 (95\% HMX) is $0.8 \mathrm{~mm} .{ }^{2}$ However, we find that the reaction zone for $\mathrm{LX}-14$ is under $0.25 \mathrm{~mm}$ while that for PETN is under $1.25 \mathrm{~mm}$. The answer is that the $1.76 \mathrm{~g} / \mathrm{cc}$ PETN is so dense that the defects are pressed out and a liquid-like overdriven state occurs. PETN of $1.71 \mathrm{~g} / \mathrm{cc}$ driven near the detonation state shows a cylinder JWL even for a $0.10 \mathrm{~mm}$ thickness, in agreement with expectations. A surprise might be that underdriven LX-14 still has a reaction zone less than $0.25 \mathrm{~mm}$ while RX-39-AC (CL-20) is larger, being less than $1.25 \mathrm{~mm}$. We summarize the spotty data by defining the (non steady-state) reaction zone thickness, $\mathbf{x}_{\mathbf{r}}$, as follows:

$\begin{array}{ll} & \underline{\underline{\mathrm{x}}} \mathbf{\mathrm { mm }}) \\ \text { PETN, } 1.71 \mathrm{~g} / \mathrm{cc} & <0.10 \\ \text { LX-14 } & <0.26 \\ \text { PETN, 1.76 g/cc } & <1.2 \\ \text { RX-39-AB (CL-20) } & <1.2 \\ \text { LX-17 } & >2.5\end{array}$

For the electric gun work, we may say that a true JWL cannot be obtained unless enough points are taken to insure being beyond the reaction zone. The flyer should be fired at near the detonation pressure. For LX-17, an ideal explosive like LX-10 must be used as the booster. The use of larger charges and thicker plates (eg. $0.5 \mathrm{~mm}$ ) should make the likelihood of success higher. If thin plates are indeed of interest, then a ZND-type burn model is needed in the code. This is the reason why no JWL ever fits LX-17.

\section{Effect of the Hugoniot on a Plate Shot}

In the Appendix, we use impedances to calculate the detonation and metal pressures at the instant of the explosive hitting a metal plate. The metal has the higher impedance and its pressure, $P_{m}$, is above the explosive detonation pressure, $P_{j}$. A reflected shock wave starts backward in the reacted explosive and creates more compression. The sum of the reflected pressure and the 
detonation pressure in the explosive equals that in the metal. For LX-14 hitting Ta, the measured free surface velocity, $u_{\mathrm{fs}}$, is $1.65 \mathrm{~mm} / \mu \mathrm{s}$ at jump-off. The metal pressure is

$$
P_{m}=\rho_{m} U_{m} u_{m} \approx \rho_{m}\left[C_{0}+S_{1}\left(u_{f s} / 2\right)\right] u_{f s} / 2 \approx 60.6 \mathrm{GPa}
$$

Here, $u_{m}$ and $U_{m}$ are the particle and wave velocities in the metal.

The reacted gas is being further compressed up its Hugoniot in the reshock. At $60.6 \mathrm{GPa}$, the volume reached is 0.633. Suppose, however, that we used the Principal Adiabat, the way all hydrocodes do. Then, at $\mathrm{v}=0.633, \mathrm{P}=55.3 \mathrm{Gpa}$, we reverse Eq. 7 to get

$$
\mathrm{u}_{\mathrm{fs}}=2 \mathrm{P}_{\mathrm{m}} / \rho_{\mathrm{m}} \mathrm{U}_{\mathrm{m}} \approx 1.51 \mathrm{~mm} / \mu \mathrm{s}
$$

A hydrocode would cause a jump-off velocity that is $8.5 \%$ low. This has been verified by Jack Reaugh, who has altered his 1-D code to convert the Principal Adiabat to the Hugoniot when $v<v_{j}$.

\section{The Expanding Wilkins' Spheres}

In 1965, expanding explosive spheres were fired to a relative volume of about $3 .{ }^{3} \mathrm{~A}$ point detonator was placed at the center, then PBX-9404 and a final spherical aluminum container. The aluminum always had an inner radius of $152.40 \mathrm{~mm}$ and an outer radius of $158.75 \mathrm{~mm}$. However, the explosive was made with different radii with a perfect no-gap fit being the fiducial. Other geometries included air gaps of 5,20 and $38.1 \mathrm{~mm}$. The idea was that the gap would allow the explosive to drop to lower pressure before it pushed, so that the resulting EOS would better define that pressure region. Wilkens believes that the cylinder, with its no-gap fit, cannot deliver a unique EOS because it sees only the higher pressure part of the pressure curve. ${ }^{4}$ He feels that the multigap approach is the only way to pin-point the true EOS.

At that time, Wilkens, et. al. used a pre-JWL EOS of the form

$$
P_{s}=\frac{a}{v^{Q}}+B\left(1-\frac{\omega}{R v}\right) \exp (-R v)+\frac{\omega E_{s}}{v}
$$

with the $\mathrm{Mb}$ constants $\mathrm{a}=0.004563, \mathrm{Q}=4, \mathrm{~B}=6.572, \mathrm{R}=4, \mathrm{w}=0.35$ and $\mathrm{E}_{\mathrm{o}}=0.1343$ for PBX-9404. We have a one-exponential JWL with a total detonation energy much larger than the actual one of 
about $0.1 \mathrm{Mb} \cdot \mathrm{cc}$. The curve has been tweaked with a negative power term to make the adjustment for the gaps. The JWL came shortly after and this EOS did not advance.

The expansion distance of the spheres was measured with a streak camera on the outer surface. This expansion, $R-R_{0}$, is shown in Fig. 6-6 for PBX-9404. As the gap expands, the pressure drops and the distance decreases. Fig. 6-7 shows some percent comparisons of the JWL runs with the data. The data was scanned from figures and it was not possible to match the time zero sections of the curves. Instead, the curves were overlaid at long times. The result was a considerable disagreement at early times, which got worse as the gap increased. In effect, the spheres experimentally did not expand as much as expected at early times but caught up later. The fits for the Wilkens' EOS's were identical to those with the cylinder JWL's for both PBX-9404 and LX-04. At the time, the Wilkens' EOS's were considered wonderful fits. ${ }^{3}$ We may say that the regular cylinder JWL works just as well on the first try, without adjustment. More importantly, the onetrack EOS works for the expanding sphere.

\section{References}

1. R. C. Weingart, R. S. Lee, R. K. Jackson and N. L. Parker, "Acceleration of Thin Flyers by Exploding Metal Foils: Application to Initiation Studies," Proceedings Sixth Symposium (International) on Detonation. Coronado, CA. August 24-27, 1976, p. 653.

2. LASL Explosive Property Data, T. R. Gibbs and A. Popolato, ed. (University of California, Berkeley, 1980), pp. 234-249.

3. M. L. Wilkens, B. Squier and B. Halperin, "Equation of State for Detonation Products of PBX 9404 and LX04-01," Tenth Symposium (International) on Combustion, p. 769 (1965); LLNL Report UCRL-7797 (1964).

4. M. L. Wilkens, Private Communication, 1995. 


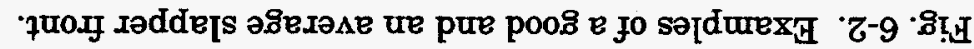

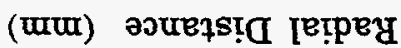

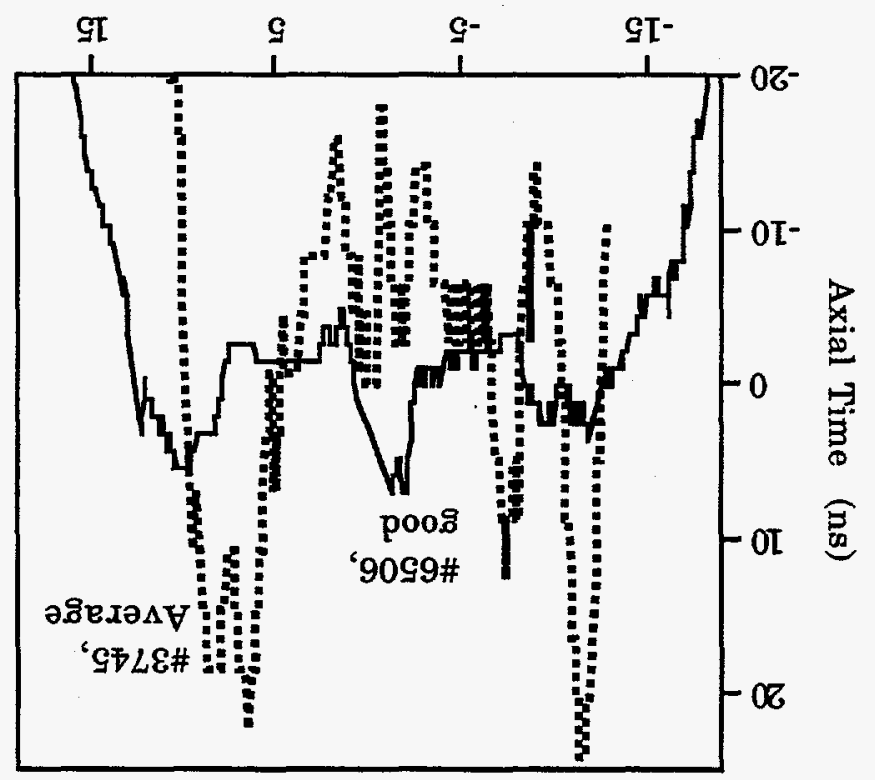

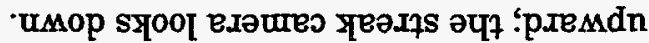

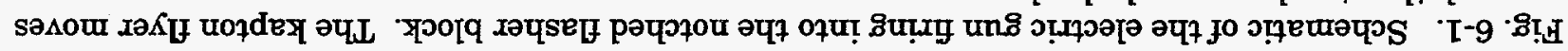

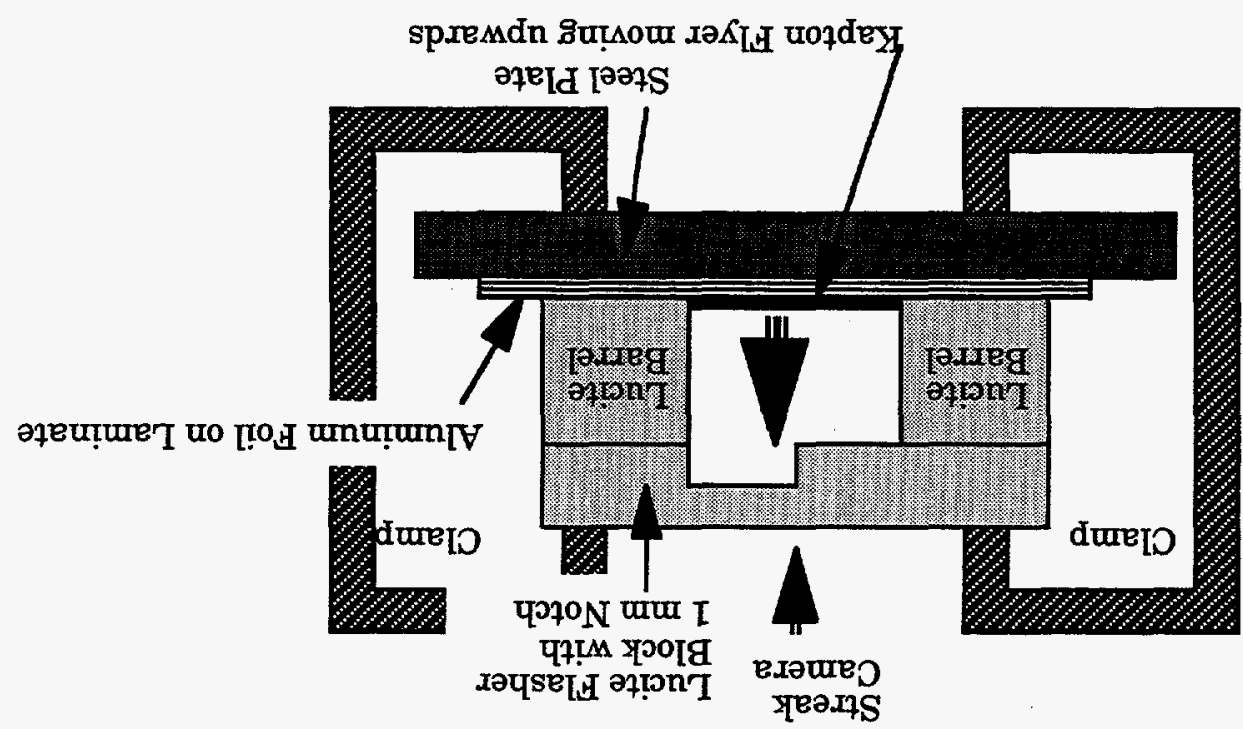




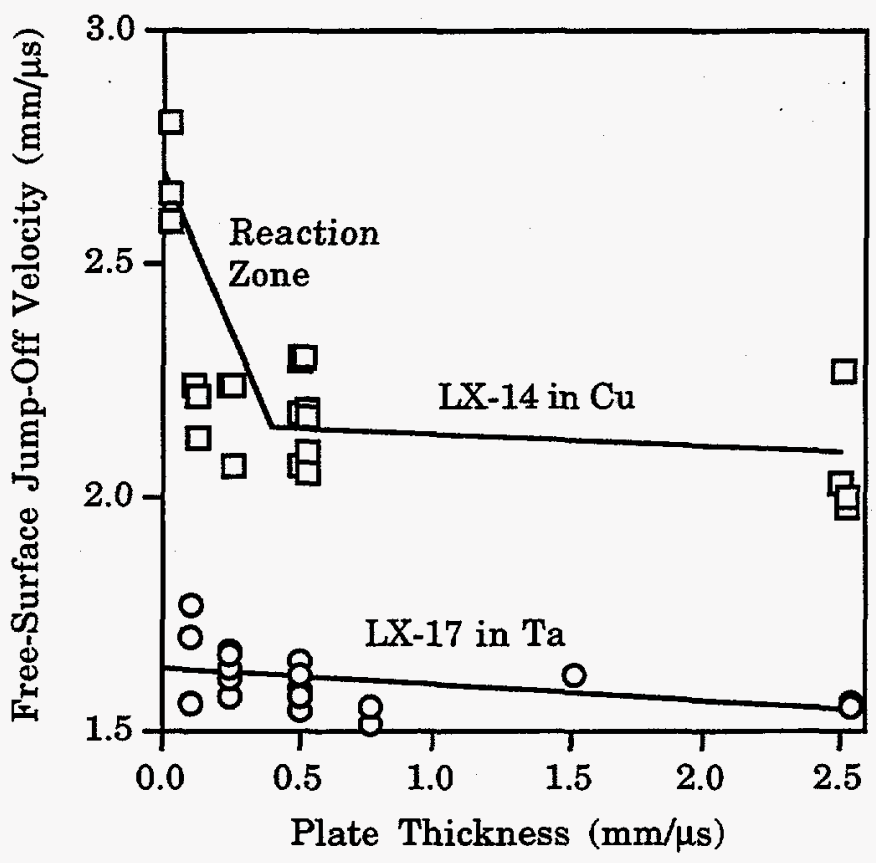

Fig. 6-3. Free-Surface jump-off velocity for $\mathrm{LX}-14$ on copper plates and $\mathrm{LX}-17$ on tantalum plates with initiation by electric gun. The quick drop for LX-14 marks the presence of the reaction zone. Beyond $0.5 \mathrm{~mm}$, the jump-off declines slowly. The LX-17 is always in its reaction zone, which is greater than $2.5 \mathrm{~mm}$ in width.

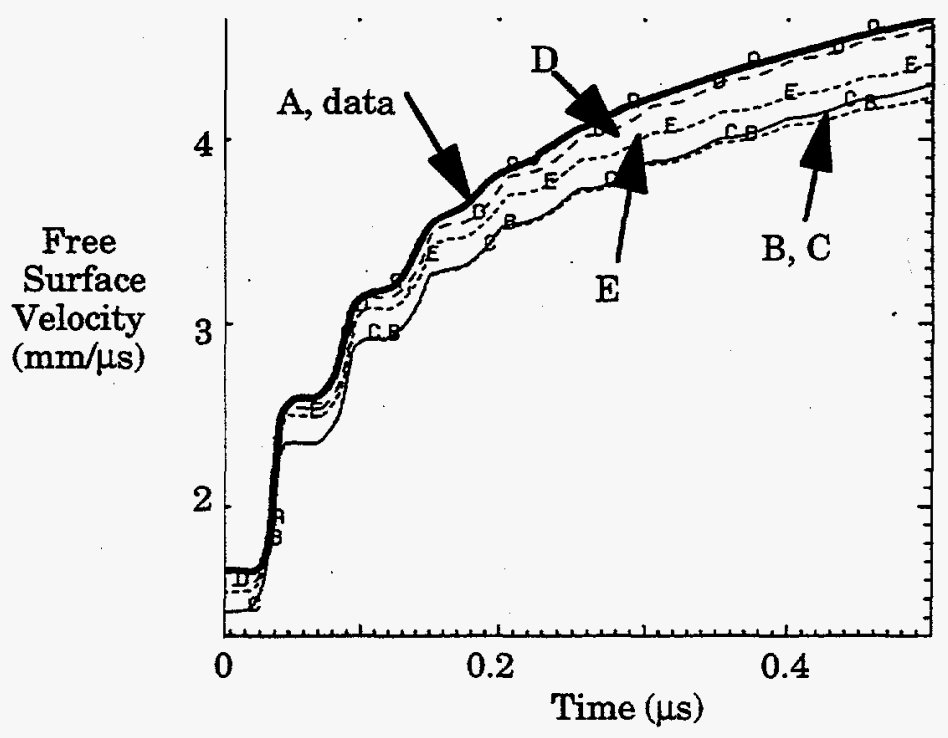

Fig. 6-4. Unconfined free face velocity for a $0.1 \mathrm{~mm}$ (4 mil) tantalum plate driven by $10 \mathrm{~mm} 1.767$ g/cc PETN. The data is higher than the cylinder JWL prediction and a more energetic JWL must be concocted. The curves, from top to bottom, are: A the data from shot ppe86001 (heavy solid line); D $\mathrm{A}=5.997, \mathrm{R}_{1}=4.5, \mathrm{R}_{2}=1.2$ (dashed); $\mathrm{E}$ 4-term JWL (dotted); $\mathrm{C} A=6.179$, the 3-term cylinder JWL (solid); and B same as before but with the flyer velocitiy set to zero (dotted). 


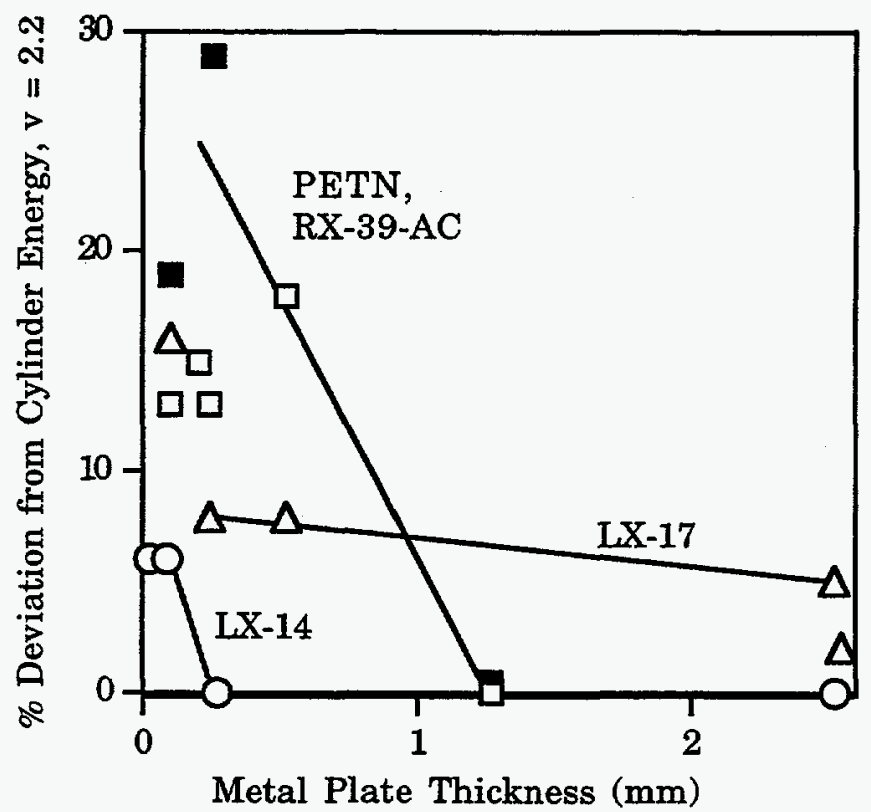

Fig. 6-5. \% deviations in enegy for plate JWL's modified to agree for reaction zone effects. Where the $\%$ goes to 0 , the JWL has no reaction zone effects and is general for the cylinder and thick plates. All data above $0 \%$ produces JWL's that fit that plate thickness only. The samples are: LX-14 (O), LX-17 ( $\Delta$ ), PETN ( $\square$ ) and RX-39-AC ( $\square$ ).

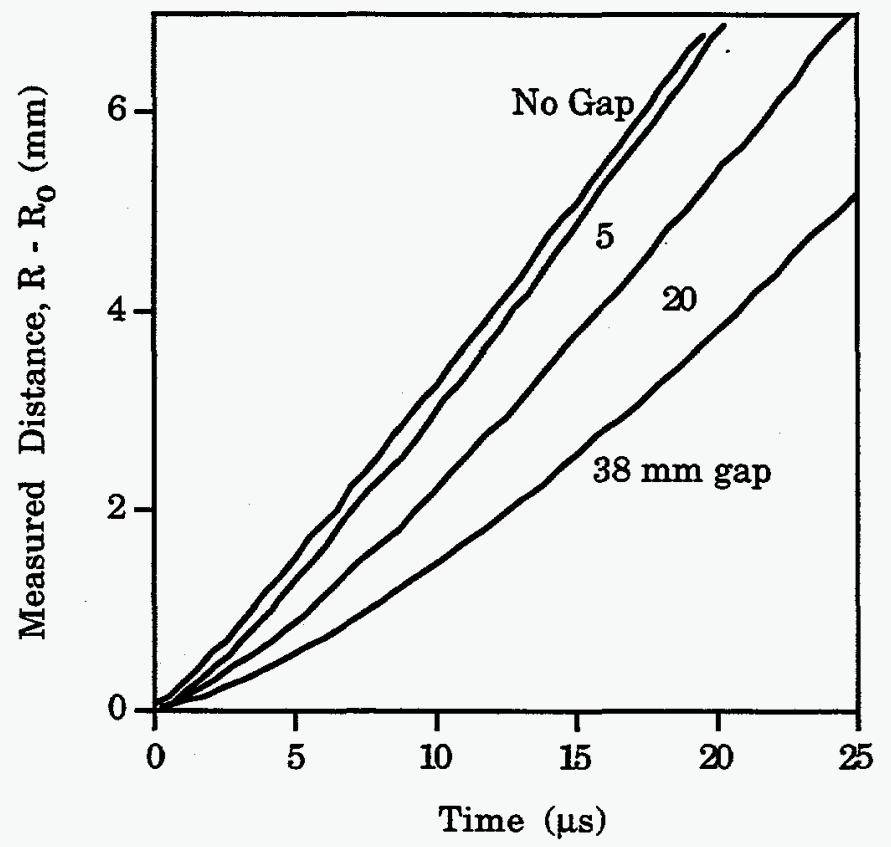

Fig. 6-6. Measured streak camera expansion distance for the outer aluminum surface of the Wilkens' spheres with PBX-9404. The larger the gap between the explosive and the inner aluminum wall, the less is the expansion distance. 


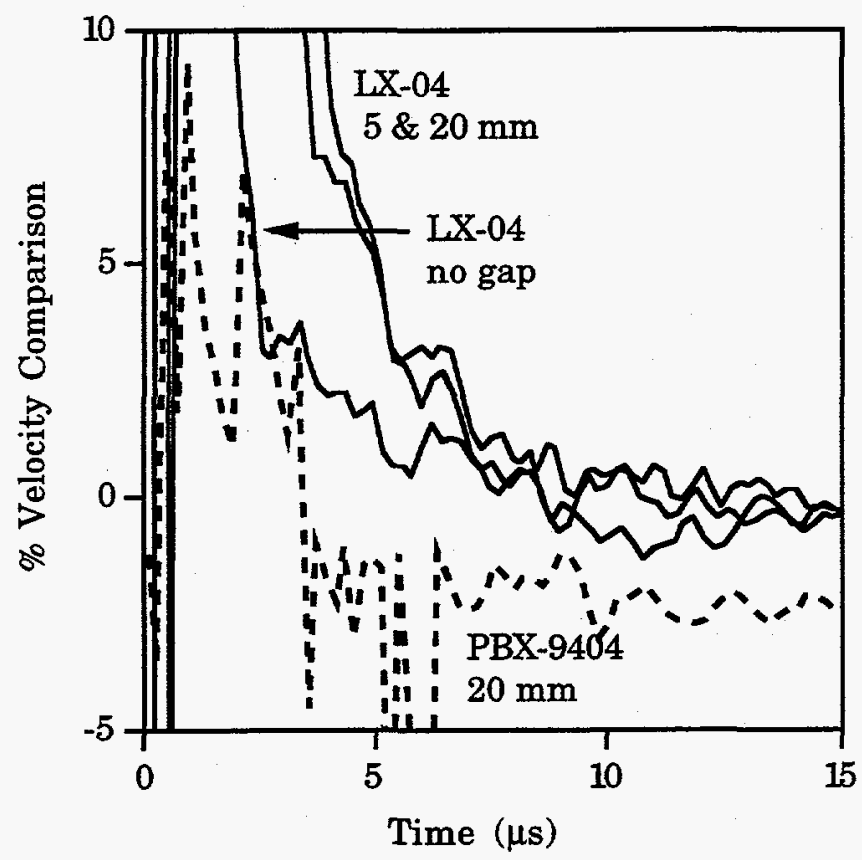

Fig. 6-7. Percent comparison of the measured streak camera distance with the JWL code. The fits are made at long time and the short-time agreement gets worse the larger the gap. The Wilkens EOS and the cylinder JWL fit both explosives equally well. 
Table 6-1. Summary of electric gun shots analyxed for agreement with cylinder JWL's. The pressures are of initiation, with bold face representing near C-J. The LX-14 and LX-17 are $51 \mathrm{~mm}$ diameters; the PETN and RX-39-AB are $25 \mathrm{~mm}$. All flyers are $0.25 \mathrm{~mm}$ (10 mil) mylar. The 97 and $99 \mathrm{~mm}$ explosives were detonated with P-40 lenses.

\begin{tabular}{|c|c|c|c|c|c|c|}
\hline \multirow[b]{2}{*}{$\begin{array}{l}\text { Explo- } \\
\text { sive }\end{array}$} & \multirow[b]{2}{*}{ Shot No. } & \multicolumn{2}{|c|}{ Thickness (mm) } & \multirow{2}{*}{$\begin{array}{l}\text { Pres- } \\
\text { sure } \\
(\mathrm{GPa})\end{array}$} & \multirow[b]{2}{*}{$\begin{array}{c}\% \mathrm{cf} \\
\nabla=2.2\end{array}$} & \multirow[b]{2}{*}{ metal } \\
\hline & & $\begin{array}{l}\text { Explo- } \\
\text { sive }\end{array}$ & Metal & & & \\
\hline LX-14 & phm85047 & 25.6 & 0.0254 & 22 & 6 & $\mathrm{Ta}$ \\
\hline \multirow[t]{4}{*}{$1.825 \mathrm{~g} / \mathrm{cc}$} & phm85001 & 5.5 & 0.095 & 22 & 6 & $\mathrm{Ta}$ \\
\hline & phm85003\&4 & 13.2 & 0.264 & 22 & 0 & $\mathrm{Ta}$ \\
\hline & phm85025,45 & 97 & 2.52 & 32 & 0 & $\mathrm{Cu}$ \\
\hline & cylinders & 210 & 2.54 & 36 & 0 & $\mathrm{Cu}$ \\
\hline PETN & ppe87002, 3 & 5.0 & 0.10 & 32 & 0 & $\mathrm{Ta}$ \\
\hline \multirow[t]{2}{*}{$1.71 \mathrm{~g} / \mathrm{cc}$} & ppe 87004,5 & 5.0 & 0.26 & 32 & 0 & $\mathrm{Ta}$ \\
\hline & ppe88003 & 9.9 & 0.26 & 19 & 13 & $\mathrm{Ta}$ \\
\hline PETN & ppe86001 & 10.0 & 0.1 & 19 & 19 & $\mathrm{Ta}$ \\
\hline \multirow[t]{2}{*}{$1.76 \mathrm{~g} / \mathrm{cc}$} & ppe86004 & 18.9 & 0.26 & 19 & $\sim 30$ & $\mathrm{Ta}$ \\
\hline & cylinder & 210 & 12.7 & 31 & 0 & $\mathrm{Cu}$ \\
\hline $\mathrm{RX}-39-\mathrm{AB}$ & pxa90004 & 5.3 & 0.096 & 22 & 13 & $\mathrm{Ta}$ \\
\hline \multirow[t]{4}{*}{$1.94 \mathrm{~g} / \mathrm{cc}$} & pxa94026 & 19.3 & 0.21 & 22 & 15 & $\mathrm{Ta}$ \\
\hline & pxa90003 & 5.3 & 0.246 & 22 & 13 & $\mathrm{Ta}$ \\
\hline & pxa90002 & 10.4 & 0.52 & 22 & 18 & $\mathrm{Cu}$ \\
\hline & cylinder & 210 & 1.27 & 40 & 0 & $\mathrm{Cu}$ \\
\hline LX-17 & pta86003 \& 8 & 19.9 & 0.102 & 20 & 16 & $\mathrm{Ta}$ \\
\hline \multirow[t]{4}{*}{$1.905 \mathrm{~g} / \mathrm{cc}$} & pta90001 & 10.1 & 0.246 & 20 & 8 & $\mathrm{Ta}$ \\
\hline & pta90003 & 1.5 & 0.52 & 20 & 8 & $\mathrm{Ta}$ \\
\hline & pta85003 \& 4 & 98.7 & 2.52 & 32 & 5 & $\mathrm{Ta}$ \\
\hline & cylinders & 210.0 & 2.54 & 27 & 2 & $\mathrm{Cu}$ \\
\hline
\end{tabular}

Table 6-2. JWL's used to fit plate shots with reaction zone behavior. The $\% \mathrm{E}_{\mathbf{d}}$ is how much more energy is present that would drive a cylinder. The PETN and RX-39-AB JWL's are good only for the plate shots they fit.

\begin{tabular}{ccc|ccc|} 
& $\begin{array}{c}\text { PETN } \\
\text { ppe86001 } \\
\text { Fig. 6-4 }\end{array}$ & $\begin{array}{c}\text { RX-39-AB } \\
\text { pxa94026 }\end{array}$ & & $\begin{array}{c}\text { PETN } \\
\text { ppe86001 } \\
\text { Fig. 6-4 }\end{array}$ & $\begin{array}{c}\text { RX-39-AB } \\
\text { pxa94026 }\end{array}$ \\
\hline$\rho_{\mathrm{o}}$ (g/cc) & 1.766 & 1.942 & $\Gamma_{\mathrm{j}}+1$ & 3.53 & 3.77 \\
$\mathrm{~A}$ (Mbars) & 5.99750 & 9.38544 & $\mathrm{Pj}$ & 0.345 & 0.438 \\
$\mathrm{~B}$ (Mbars) & 0.23067 & 0.18771 & $\mathrm{D}$ & 0.8283 & 0.9208 \\
$\mathrm{C}$ (Mbars) & 0.00706 & 0.00297 & $\mathrm{vj}$ & 0.717 & 0.735 \\
$\mathrm{R}_{1}$ & 4.50 & 4.50 & $\% \mathrm{E}_{\mathrm{d}}$ & & \\
$\mathrm{R}_{2}$ & 1.2 & 1.0 & at $\mathrm{v}=2.2$ & 21 & 15 \\
$\omega$ & 0.29 & 0.34 & at $\mathrm{v}=4.1$ & 22 & 21 \\
$\mathrm{E}_{\mathrm{o}}(\mathrm{Mb} * \mathrm{cc})$ & 0.112 & 0.118 & at $\mathrm{v}=6.5$ & 18 & 19
\end{tabular}




\section{Chapter 7. Imploding Spheres}

\section{Compression and the Hugoniot}

Almost exclusive attention is given to the Principal Adiabat of a given reacted explosive. This is because almost all explosives are under-initiated. By this, we mean that the initial Rayleigh Line is below the Principal Rayleigh Line. Most explosives are hit hard enough that the difference is quickly made up by the run-away action of the explosive itself. There is no practical need to consider the over-driven explosive.

One geometry forces us to break this habit: the compressed sphere. Here, the energy is put into an ever smaller volume so that the detonation velocity increases and the explosive becomes overdriven. Then, the maximum pressure points do not lie on an adiabat but on the reacted Hugoniot. P-v space in a compressed sphere PBX-9404 from $0.55<\mathrm{v}<1.0$ is shown in Fig. 7-1. In HEMP, we looking at the rise in pressure and pressure-plus-artificial viscosity, $P+Q$, in a single cell that is being overdriven. The end point of highest pressure sits on the reacted Hugoniot above the detonation point. When release occurs, this cell will descend a different adiabat than the Principal Adiabat, which is anchored at the detonation point. The deeper into the sphere, the higher in pressure the end point will be. Each cell will have a different release adiabat based on its radial position. This is a true multi-track EOS problem.

\section{Reacted Hugoniot Data from Supracompression}

There are huge quantities of data on the Principal Adiabat but very little for the reacted Hugoniot. The only data is from supracompression experiments. ${ }^{1-6}$ In the most recent LLNL shots, the two-stage gas gun was used to generate pressures at or above C-J. The aluminum sabot diameter was $25 \mathrm{~mm} .{ }^{6}$ A terraced explosive sample was used and $U_{\mathrm{S}}$ was directly measured from pins on adjacent terraces. The less definitive result was the particle velocity $u_{p}$, which was obtained from impedance matching calculations, given the initial velocity of the sabot. As shown in Appendix $A$, the $u_{p}$ result can vary by \pm 3 to $\pm 5 \%$ depending on the choice of the $U_{S}-u_{p}$ EOS and whether it represents the reacted or unreacted explosive. Because of this uncertainty, $u_{p}$ should be directly gauge-measured should supracompressed Hugoniots ever again be measured. The initial velocity, however, was listed as being known to $\pm 0.1 \%$ because of the taking of two pulsed X-ray pictures with 
the impactor in flight. 5 After an initial spike, the large sabot created a pressure plateau stable for several $\mu$ s, although the $U_{\mathrm{S}}$ measurement must be taken before the side effects eat in. 4

We are assuming here that the states of both the compressed sphere and the overdriven planar gun shot are the same and are described by the reacted Hugoniot. Craig Tarver agrees with this but notes that there is probably a kinetic difference between the two experiments. The sphere compresses so quickly that carbon coagulation has no time to take place. The two Hugoniots are, therefore, probably slightly different, but there is no way to distinquish between them at this time.

The usual Rankine-Hugoniot equations we use for the C-J point is for a frictionless piston that allows the reaction products to pass through it. Here, the sabot is a real piston that piles up all the mass in front of it. The real piston can create a wave velocity, $U_{\mathbf{S}}$, that is greater than the explosive's steady state detonation velocity. We have

$$
\mathrm{v}_{\mathrm{h}}=1-\mathrm{u}_{\mathrm{p}} / \mathrm{U}_{\mathrm{s}}
$$

and

$$
P_{h}=\rho_{o} U_{s}^{2}\left(1-v_{h}\right)
$$

From this, we can fit the function $\mathrm{P}_{h}\left(\mathrm{v}_{h}\right)$. The energy of compression at any point on the Hugoniot is

$$
E_{c}=\frac{1}{2} P_{h}\left(1-v_{h}\right)
$$

The chemical energy is the same at $\mathrm{E}_{0}$, so that the total internal energy is

$$
\mathrm{E}_{\mathrm{h}}=\mathrm{E}_{\mathbf{0}}+\mathrm{E}_{\mathrm{c}}
$$

This becomes

$$
-\int\left(P_{h}+Q_{h}\right) d v=\frac{1}{2} P_{h}\left(1-v_{h}\right)+E_{0}
$$

Most folks like to hand-massage their EOS's and Eq. 5 is what they need to do the integration. Eq. 5 is mathematical on the right side with a non-physical $Q$ on the left to make the integration work. 
Initially, in a code, $Q$ pushes and causes acceleration. Later, $Q$ decreases as $P$ grows so that the maximum pressure is indeed $\mathrm{Ph}_{\mathrm{h}}$. This is why $\mathrm{Q}$ does not appear on the right side of Eq. 5 .

\section{Hugoniot EOS's for LX-14 and Others}

We first consider the pressure vs. volume results. The Hugoniots have been run in CHEETAH1.0 and CHEQ2.5 and compared with the supracompression data. ${ }^{1-5}$ The results for four explosives are shown in Fig. 7-2. The data is generally lower than the CHEQ results and often agrees with CHEETAH at lower pressures. The CHEQ results fit as well for PBX-9404 but progressively deviate as we move from HMX toward TATB, ie. in the order PBX-9404 < PETN $<$ RX-26-AF < LX17. Unfortunately, only two data points have been taken for LX-14, but they are close to the PBX9404 curve. ${ }^{5}$ However, CHEETAH clearly does not have the right curve-shape and it undercalculates pressures at the small volumes. So the CHEQ curve-shapes appear to be correct but the curves need to be reset downwards.

The systematic error in the data needed to make the data agree with CHEQ could occur in experimental $u_{p}$, which is itself calculated from impedance. If all values of $u_{p}$ were lowered by $5 \%$, a large but believable amount, we would have agreement.

We have derived in Table 7-1 the compressive "Hugoniot" JWL's for six explosives. The method used was as follows. The combination of $R_{1}=7.7$ and $R_{2}=2.4$ was carried over unchanged from the PBX-9404 case. The Principal Adiabat values of $\omega$ and $E_{0}(\infty)$ were also used. along with the three cylinder detonation energies at volumes of 2.2, 4.1 and 6.5. Then A and B were raised and the JWL is fitted to match the cylinder energies. The calculated $P-v$ curve was compared with measured supracompression data and CHEQ runs. The calculated curve was always expected to lie a few percent below the CHEQ curve. If the result was not good, $\Gamma_{\mathrm{j}}+1$ was changed and the process repeated. The resulting JWL's match all the available data. An explosive like RX-39-AB was believable because its JWL lay just below its calculated CHEQ curve and the explosive is ideal and much like LX-14 in its properties. The hardest to fit, as expected, was LX-17 with its non-ideal behavior.

It should be emphasized that this mongrol process is completely empirical, but it does succeed in grafting a Hugoniot for $\mathrm{v}<\mathrm{v}_{j}$ onto a Principal Adiabat-like curve. These JWL's are sizedependent, for as the explosive becomes small in size, we would expect its JWL to approach our usual $R_{1}=4.5$ Principal Adiabat curve. Our class of $R_{1}=7.7$ curves are an accidental result of the size and thickness of the spherical explosive that was used to generate the data. 
Another issue is considered in Fig. 7-3, where adiabatic $\Gamma$ is plotted. The old Hugoniot was set to have a very flat slope in the above-CJ region. The new Hugoniot has a slope much like the adiabat JWL it copies. The extrapolated adiabat is also shown below the other two. The supracompression data has been smoothed and differentiated, and the results show that the extrapolated asiabat is probably not the right answer. However, the scatter in the data is too great to select the right Hugoniot.

We note that using Ignition \& Growth (Reactive Flow) does not change anything. Although unreacted explosive is added, the net result is still the Principal Adiabat, below the detonation point and the Rayleigh Line above. I\&G is not able to climb up the Hugoniot or to come down an overdriven adiabat.

\section{The French Compressed PBX-9404 Sphere}

Four spheres of plastic-bonded HMX of density $1.836 \mathrm{~g} / \mathrm{cc}$, essentially PBX-9404 but close to LX-14, were fired from the outside surface by Cheret, et. al. ${ }^{7}$ Pins set at various depths give the arrival of the detonation wave. The detonation velocities are calculated between points and the distance runs from the outer surface inwards. From Fig. 7-4, we see that the two $140 \mathrm{~mm}$ radius spheres start at the expected $8.8 \mathrm{~mm} / \mu$ s velocities, but the $150 \mathrm{~mm}$ samples start more slowly. This continues at later times, where the $140 \mathrm{~mm}$ velocities are faster. The under-initiated $150 \mathrm{~mm}$ spheres never catch up. Hence, we shall take the $280 \mathrm{~mm}$ samples as being correct. The detailed method of initiation is not listed in the report. The times at each of the pins is listed but there is no absolute timing relative to initiation on the surface.

The $140 \mathrm{~mm}$ radius \#1 sphere was run in the 1-D code to match the timing of the experiments. Monotonic $Q$ was used. An extra $1 \mathrm{~mm}$ of explosive was added to the outside and volume-burned to serve as a detonator. The pressure increased steadily with no droop occuring just after initiation using this scheme. All calculated times were referenced to the first inside pin. The detailed results are listed in Table 7-2. The time differences are added up as a measure of the fit. The smallest and best number is for the $\mathrm{A}=38.00$ Hugoniot derived above.

\section{Measuring High Pressure Adiabats}

As mentioned above, there will be an infinite number of adiabats for every sphericallycompressed explosive. If a piece of metal moves after compression, we now need to deal with these 
adiabats. Such experiments are rare but one done by Harry Vantine and Don Griswold (Larry Simmons, ramrod) is schematically shown in Fig. 7-5. The system was an inward-driven sphere with LX-14 pushing a steel plate. The LX-14 had an outer radius of $181.02 \mathrm{~mm}$ and an inner radius of $88.76 \mathrm{~mm}$; the steel radii were 88.76 and $86.03 \mathrm{~mm}$, respectively. Fabry-Perot velocity measurements were taken by Rex Avara on the unconfined steel face. Also, a time was measured between the jumpoff of the steel plate and the light from the flasher block, set at a radius of $47.38 \mathrm{~mm}$. In modeling in 1-D, the rapid jump-off of the flasher block is taken, not the slow start caused by the pile-up of the hot gas. In the code written by Don Griswold, the explosive was given 6.8 zones $/ \mathrm{mm}$ with a volume burn being used in the outer $1 \mathrm{~mm}$ ( 7 zones). The pressure increased steadily with no droop occuring just after initiation using this scheme. The steel had 8.4 zones/mm and the Steinberg-Guinan material model was used, there being no evidence of spall in the data. Running the Cochran-Banner model with spall produced no change.

The results are summarized below:

\begin{tabular}{lcc}
\multicolumn{1}{c}{ EOS } & $\begin{array}{c}\text { Jump-Off } \\
\text { Velocity } \\
(\mathrm{mm} / \mu \mathrm{s})\end{array}$ & $\begin{array}{c}\text { Steel-to- } \\
\text { Flasher } \\
\text { Time }(\mu \mathrm{s})\end{array}$ \\
\hline Measured & 2.82 & 7.72 \\
$\mathrm{~A}=38.00$ Hugoniot & 2.98 & 7.77 \\
$\mathrm{~A}=$ 41.85 Hugo/9404 & 3.01 & 7.69 \\
$\mathrm{~A}=7.65$ Adiabat & 3.15 & 7.50
\end{tabular}

The measured jump-off velocity of $2.82 \pm 0.14 \mathrm{~mm} / \mu \mathrm{s}$ is low no matter what EOS is used. We have looked for a low jump-off coupled with a steel-to-flasher time of about the right value. We note some uncertainties in this experiment. An estimated $\pm 0.25 \mu$ sitter existed in the lens so that timing to the start of initiation was not feasible. A pin inside the explosive would have helped establish a zero time earlier than the steel jump-off. The shock front going into the $\mathrm{LX}-14$ and at least $10 \mathrm{~mm}$ inside was known from previous work to be ragged, with a width of 1.5 to $2.0 \mathrm{~mm}$.

The Fabry results are shown in Fig. 7-6. The code results are definitely different between the $\mathrm{A}=7.65$ adiabat and the $\mathrm{A}=38.0$ Hugoniot, showing that the use of the Hugoniot is correct. The latter agrees at jump-off with the highest of the nine Fabry measurements but is slightly above the average. The agreement becomes good at later times. 
Why is the Hugoniot with $R_{1}=7.7$ so different from the $R_{1}=4.5$ adiabat? The answer is that we can put only one equation into the code and we are asking it to do two different things. The first thing is to construct a true Hugoniot as in the French sphere with pins inside the explosive. The second thing is to describe the high pressure adiabats driving a plate in spherical geometry. The final JWL does both these jobs and sums over all possible high pressure adiabats.

\section{Calculating High Pressure Adiabats for LX-14 (with Al Nichols)}

High pressure adiabats for LX-14 were run using the CHEQ code with the $1800 \mathrm{~K}$ freeze. These are really curves at different constant entropies. A Hugoniot is needed to find the intersection with each adiabat. The Prinicipal Adiabat may be considered as a special Hugoniot case at the C-J point of $0.347 \mathrm{Mb}$. We selected three more Hugoniot points at pressures, $\mathrm{P}_{\mathrm{h}}$, of $0.53,0.73$ and 1.00 Mb. Using also the Hugoniot volumes, $v_{h}$, the detonation velocities, $U_{S}$, are calculated from the equation

$$
\mathrm{U}_{\mathrm{s}}=\left[\frac{\dot{\mathrm{P}}_{\mathrm{h}}}{\rho_{\mathrm{o}}\left(1-\mathrm{v}_{\mathrm{h}}\right)}\right]^{1 / 2} \text {. }
$$

This is the intersection of the Hugoniot and the Rayleigh Line but without the C-J condition of tangency. Fig. 7-7 shows the results. Each adiabat is a separate curve to be tracked in a code.

It is possible to create JWL's for these adiabats. Using the method of Chapter 2, we derive $\omega$ and $E_{0}(\infty)$. We see in Table 7-3 that they are almost but not quite constant as the Hugoniot pressure increases.

We consider the Hugoniot point $\left(\mathrm{P}_{\mathrm{h}}, \mathrm{V}_{\mathrm{h}}\right)$ to be a detonation point and we start the JWL downward in pressure from there.

$$
P_{h}=A \exp \left(-R_{1} v_{h}\right)+B \exp \left(-R_{2} v_{h}\right)+\frac{\omega}{v_{h}}\left[E_{o}(\infty)-E_{c}\right]
$$

Here, $\Gamma_{j}+1$ is not equal to $1 /\left(1-v_{h}\right)$ because of the lack of tangency to the Rayleigh Line. It has to be calculated directly from the JWL. The coefficient $\mathrm{C}$ comes from

$$
C=v_{h}^{1+\omega}\left[P_{h}-A \exp \left(-R_{1} v_{h}\right)-B \exp \left(-R_{2} v_{h}\right)\right]
$$


We have set $\mathrm{Ph}_{\mathrm{h}}$ and $\mathrm{vh}$ absolutely at the detonation point. Now we slide down the adiabat to the three cylinder volumes of $2.2,4.1$ and 6.5 where we calculate the detonation energies

$$
E_{d}(v)=E_{o}(\infty)-\left[\left(\frac{A}{R_{1}}\right) \exp \left(-R_{1} v\right)+\left(\frac{B}{R_{2}}\right) \exp \left(-R_{2} v\right)+\frac{C}{\omega v^{\omega}}\right] .
$$

The three cylinder points are so clustered that they really count as one, so the problem, without a tangential Rayleigh Line, is underdetermined. We, therefore, set $\omega$ and $E_{0}(\infty)$ constant and set $\mathrm{A}$ and $\mathrm{B}$ proportional to the initial Hugoniot pressure. The resulting JWL's in Table 7-4 are one possible set, which reproduces the curves closely enough to show how the JWL's look. There are a large number of fitting problems. First, the various models have a considerable slippage in their values. For example, the CHEQ C-J pressure is $0.347 \mathrm{Mb}$ while we generally use $0.373 \mathrm{Mb}$ in the cylinder JWL. CHEQ's detonation velocity is $0.897 \mathrm{~cm} / \mu \mathrm{s}$ whereas $0.878 \mathrm{~cm} / \mu \mathrm{s}$ is the measured value. The next fit is in connecting the Hugoniot seamlessly with the Principal Adiabat and the various high-pressure adiabats. Some sort of general JWL adiabat equation would have to be devised. Connecting these to the Hugoniot would be difficult.

It is possibly surprising to note that the cylinder energies are less as the adiabats start higher up the Hugoniot. This occurs because the compression is great and the initial energy is more negative. The energy has farther to go to reach the positive values we are used to.

\section{References}

1. P. C. Souers and L. C. Haselman, Jr., Detonation Equation of State at LLNL, 1993, LLNL Report UCRL-ID-116113 (March, 1994), pp. 6-8 to 6-13, 6-25 to 6-27.

2 J. H. Kinecke, Jr. and C. E. West, Jr., "Shocked States of Four Overdriven Explosives," Proceedings Fifth Symposium (International) on Detonation, Pasadena, CA, August 18-21, 1970, pp. 533-542.

3. E. L. Lee, M. van Thiel, L. G. Green and A. Mitchell, Detonation Product EOS: The Region above Chapman Jouget Pressure, LLNL Report UCRL-88983 (1983); also given at the American Physical Society 1983 Topical Conference on Shock Waves in Condensed Matter, Santa Fe, NM, July 18-21, 1983.

4. L. Green, E. Lee, A. Mitchell and C. Tarver, "The Supra-Compression of LX-07, LX-17, PBX-9404, and RX-26-AF and the Equations of State of the Detonation Products," Proceedings Eighth Symposium (International) on Detonation, Albuquerque, NM, July 15-19, 1985, pp. 587-595.

5. L. Green, N. Holmes and J. Kury, "Shock Measurements on Explosives in the Supra-Compressive Region," International Symposium on Pyrotechnics and Explosives, Beijing, China, October 12-15, 1987; LLNL Report UCRL-95461 (1987). 
6. L. C. Green, C. M. Tarver and D. J. Erskine, "Reaction Zone Structure in Supracompressed

Detonating Explosive," Proceedings Ninth Symposium (International) on Detonation. Portland, OR, August 28-September 1, 1989, p. 670.

7. R. Cheret, F. Chaisse and J. Zoe, "Some Results on the Converging Spherical detonation in a Solid Explosive," Proceedings Seventh Symposium (International) on Detonation, Annapolis, MD, June 1619,1981, p. 602 .

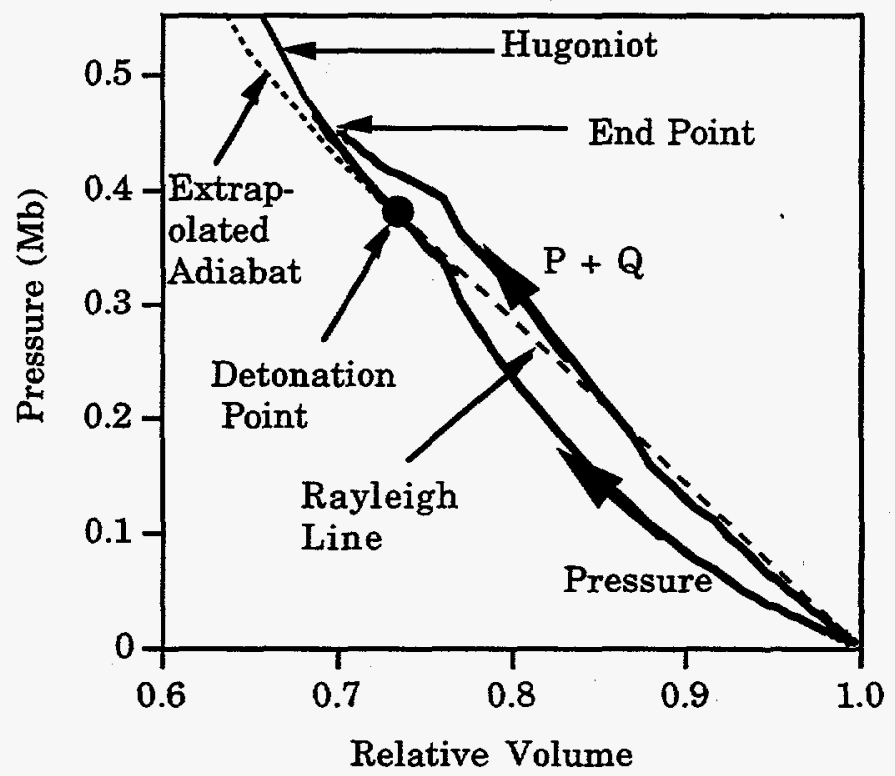

Fig. 7-1. Track of HEMP code pressure in a single cell inside a compressed PBX-9404 sphere. The state is overdriven and lies on the input EOS Hugoniot, which is higher in pressure than the detonation point. The pressure rises just below the Rayleigh Line to the detonation point, after which it follows the Hugoniot. The total pressure, $P+Q$ lies below the Rayleigh Line, then moves above and finally comes to rest at the same point on the Hugoniot. A new adiabat will start downward from the end point on the Hugoniot. 

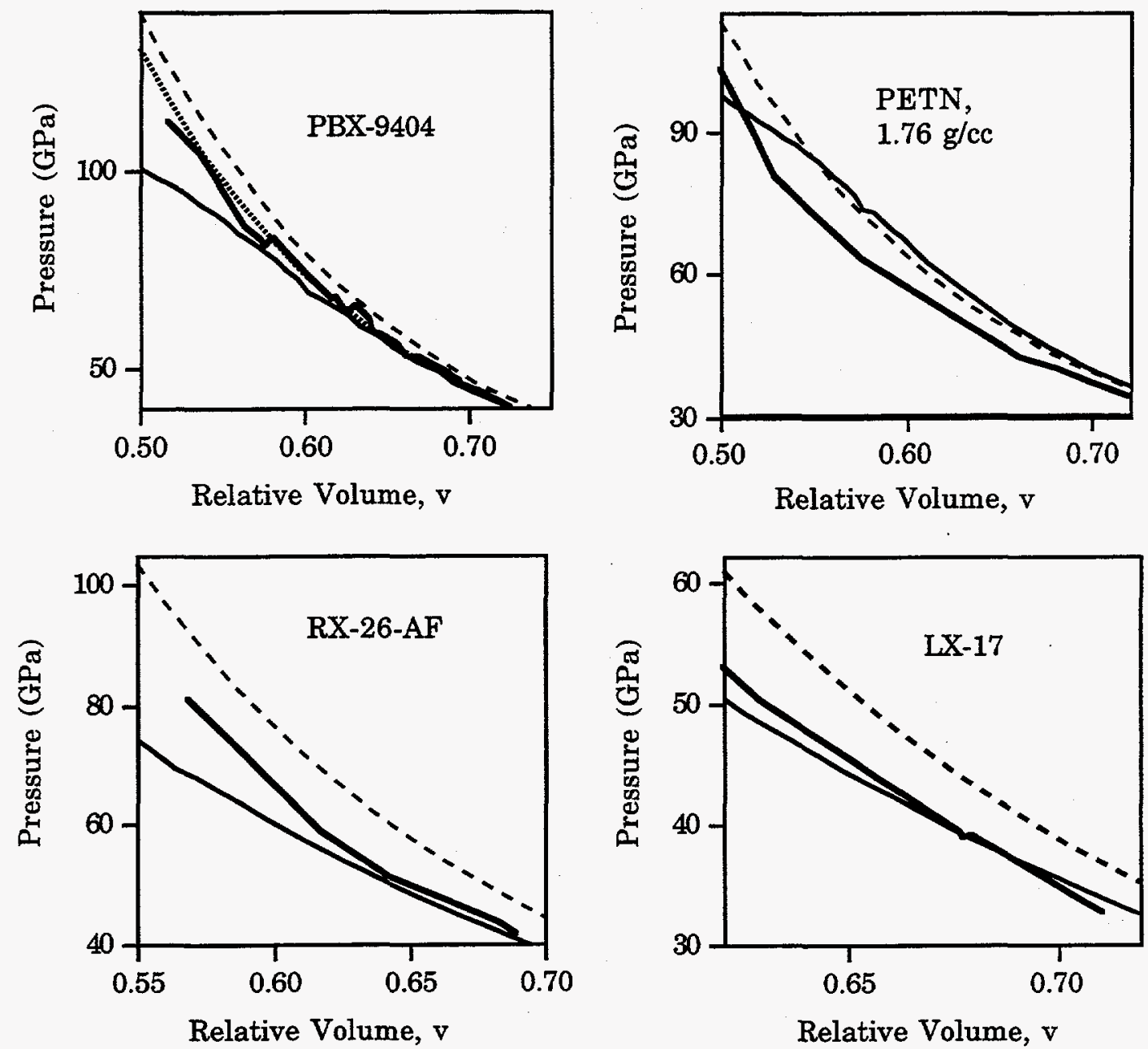

Fig. 7-2. Pressure-volume Hugoniots for four explosives progressing from the most to the least ideal. The lines are: data (heavy line), CHEETAH (solid line), and CHEQ (dashed line). The measured values are lower than the CHEQ values but have the same upward curvature. The dotted line for PBX-9404 is the old JWL. 


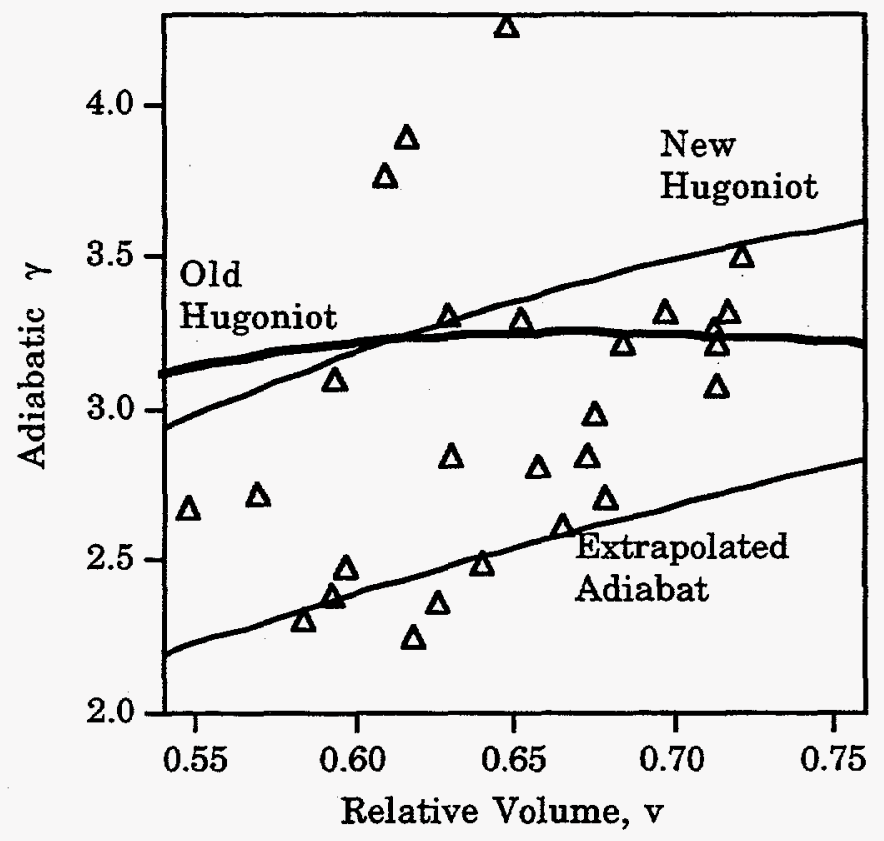

Fig. 7-3. Adiabatic $\Gamma$ for the old and new Hugoniot plus the extrapolated adiabat. The old Hugoniot has a very flat slope. Getting an answer from the differentiated supracompression data (triangles) is a hopeless task.

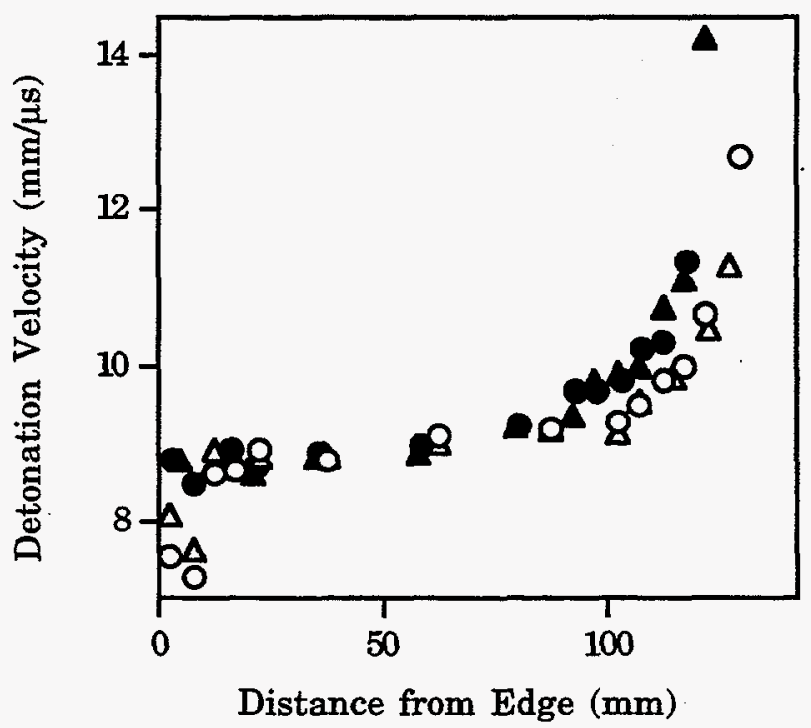

Fig. 7-4. Detonation velocities found from pins inside the four French $1.836 \mathrm{~g} / \mathrm{cc}$ plastic-bonded HMX spheres. The closed symbols are from the two $140 \mathrm{~mm}$ radius spheres, which initiated perfectly. The open symbols are from the $150 \mathrm{~mm}$ spheres, which were underinitiated., 


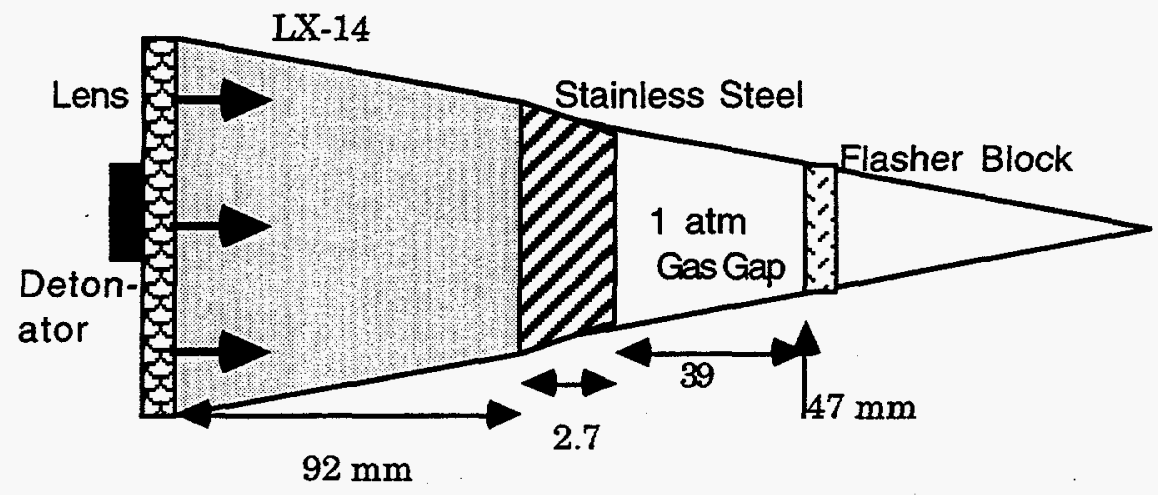

Fig. 7-5. Schematic of the spherical inward-burn compression experiment by Vantine and Griswold. LX-14 drives a steel plate. Detonation moves from left to right.

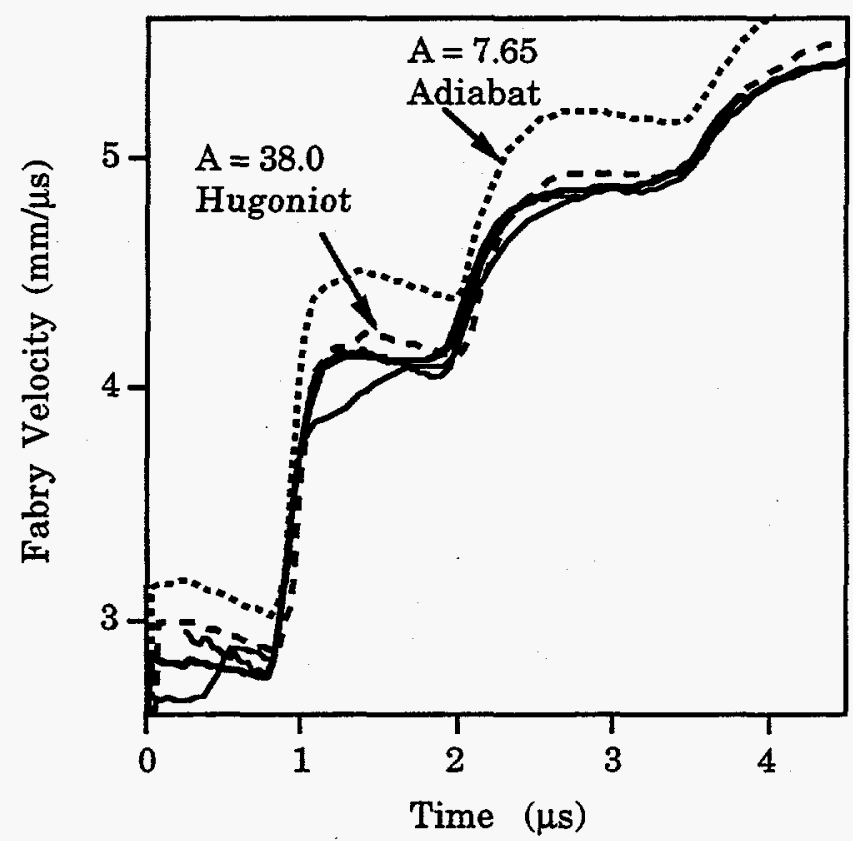

Fig. 7-6. Comparison of the 1-D code with Fabry data from the inward-burning spherical plate push by Vantine and Griswold. The data average is the heavy line, with the two extremes of the nine cameras shown in light full lines. The code results using the $A=7.65$ adiabat (dotted) and the $A=$ 38.0 Hugoniot (dashed) show that the two may be distinquished. 


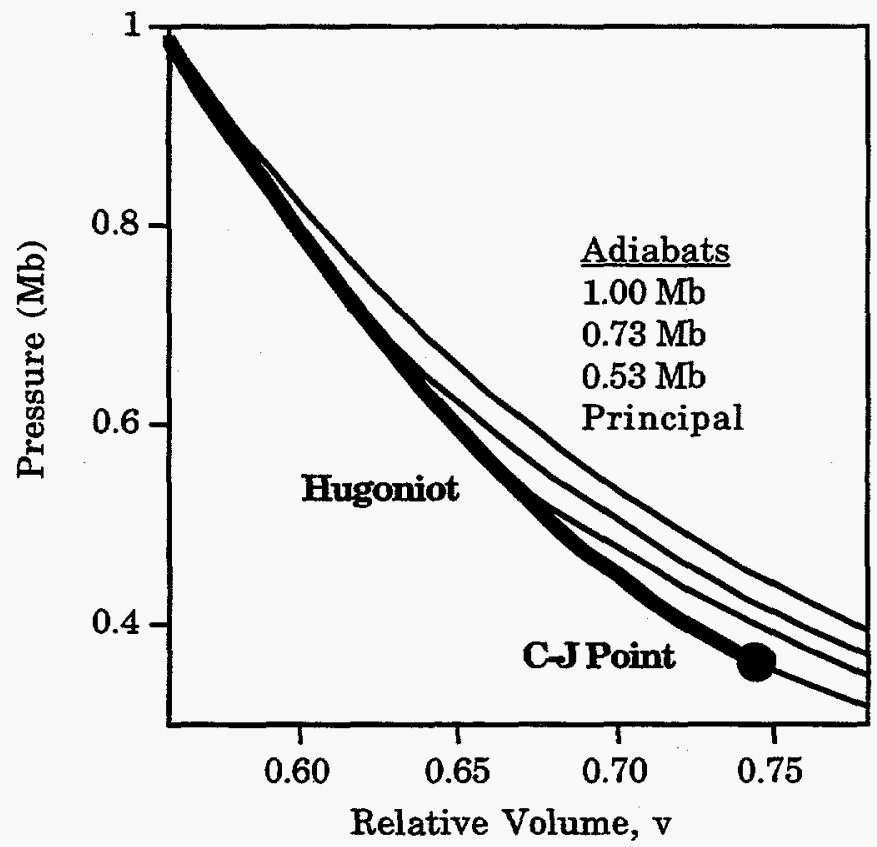

Fig. 7-7. Calculated LX-14 adiabats as they spring from the Hugoniot. The Hugoniot pressures are listed for the curves from top to bottom.

Table 7-1. Set of compression "Hugoniot" JWL's for various explosives in Mb units.

\begin{tabular}{ccccccc} 
& LX-14 & LX-17 & PBX-9404 & PETN & RX-26-AF & RX-39-AC \\
\cline { 2 - 6 }$\rho_{0}(\mathrm{~g} / \mathrm{cc})$ & 1.825 & 1.90 & 1.84 & 1.766 & 1.844 & 1.942 \\
$\mathrm{~A}(\mathrm{Mb})$ & 38.0000 & 36.5300 & 41.8548 & 25.2900 & 42.1950 & 46.8680 \\
$\mathrm{~B}(\mathrm{Mb})$ & 1.2955 & 0.9317 & 1.2553 & 1.2328 & 0.9933 & 1.4810 \\
$\mathrm{C}(\mathrm{Mb})$ & 0.013639 & 0.005881 & 0.014828 & 0.014609 & 0.013682 & 0.014978 \\
$\mathrm{R}_{1}$ & 7.7 & 7.7 & 7.7 & 7.7 & 7.7 & 7.7 \\
$\mathrm{R}_{2}$ & 2.4 & 2.4 & 2.4 & 2.4 & 2.4 & 2.4 \\
$\omega$ & 0.33 & 0.26 & 0.38 & 0.29 & 0.30 & 0.34 \\
$\mathrm{E}_{\mathrm{o}}(\mathrm{Mb} . \mathrm{cc} / \mathrm{cc})$ & 0.1060 & 0.0690 & 0.1020 & 0.1120 & 0.0960 & 0.1180 \\
$\Gamma_{\mathbf{j}}+1$ & 4.10 & 4.40 & 4.20 & 3.80 & 4.40 & 4.17 \\
$\mathrm{D}(\mathrm{cm} / \mu \mathrm{s})$ & 0.8783 & 0.7596 & 0.8832 & 0.8283 & 0.8240 & 0.9210 \\
$\mathrm{P}_{\mathbf{j}}(\mathrm{Mb})$ & 0.343 & 0.249 & 0.342 & 0.319 & 0.285 & 0.395 \\
$\mathrm{v}_{\mathbf{j}}$ & 0.756 & 0.773 & 0.762 & 0.737 & 0.773 & 0.760
\end{tabular}


Table 7-2. Comparison of 1-D code runs with the timing data from the French $1.836 \mathrm{~g} / \mathrm{cc}$ plasticbonded HMX $140 \mathrm{~mm}$ radius sphere \#1. The fit is described by the smallness of the sum of timing differences. The best fit is given by the $\mathrm{A}=38.00$ Hugoniot intended for LX-14.

\begin{tabular}{|c|c|c|c|c|c|c|}
\hline \multirow{2}{*}{$\begin{array}{c}\text { Measd } \\
\text { Radius } \\
(\mathrm{mm})\end{array}$} & \multirow{2}{*}{$\begin{array}{c}\text { Measd } \\
\text { time } \\
(\mu s)\end{array}$} & \multirow{2}{*}{$\begin{array}{l}\text { Zones } \\
\text { in from } \\
\text { center }\end{array}$} & \multirow{2}{*}{$\begin{array}{c}\text { Measd } \\
\text { Zero } \\
\text { Time } \\
(\mu s)\end{array}$} & \multicolumn{3}{|c|}{ Time Difference $(\mu s)$} \\
\hline & & & & $\begin{array}{l}\mathrm{A}=7.65 \\
\text { Adiabat }\end{array}$ & $\begin{array}{l}\mathrm{A}=41.85 \\
\text { Hugoniot }\end{array}$ & $\begin{array}{l}A=38.00 \\
\text { Hugoniot }\end{array}$ \\
\hline 140.00 & 13.838 & 560 & & & & \\
\hline 134.83 & 14.425 & 539 & 0.00 & 0.00 & 0.00 & 0.00 \\
\hline 130.20 & 14.970 & 520 & 0.55 & -0.02 & 0.00 & 0.01 \\
\hline 125.98 & 15.460 & 503 & 1.04 & -0.03 & -0.02 & 0.00 \\
\hline 120.92 & 16.025 & 483 & 1.60 & -0.03 & -0.02 & 0.00 \\
\hline 116.30 & 16.555 & 465 & 2.13 & -0.05 & -0.04 & -0.02 \\
\hline 92.84 & 19.190 & 371 & 4.77 & -0.02 & -0.03 & 0.01 \\
\hline 70.03 & 21.725 & 280 & 7.30 & 0.01 & -0.04 & 0.01 \\
\hline 50.10 & 23.880 & 200 & 9.46 & 0.06 & -0.04 & 0.02 \\
\hline 45.06 & 24.400 & 180 & 9.98 & 0.07 & -0.04 & 0.02 \\
\hline 39.98 & 24.925 & 159 & 10.50 & 0.10 & -0.04 & 0.03 \\
\hline 35.01 & 25.430 & 140 & 11.01 & 0.09 & -0.07 & 0.00 \\
\hline 30.05 & 25.915 & 120 & 11.49 & 0.11 & -0.08 & 0.01 \\
\hline 25.04 & 26.400 & 100 & 11.98 & 0.11 & -0.10 & -0.01 \\
\hline 20.04 & 26.840 & 80 & 12.42 & 0.15 & -0.10 & -0.01 \\
\hline 15.02 & 27.200 & 60 & 12.78 & 0.24 & -0.05 & 0.04 \\
\hline \multicolumn{2}{|c|}{ Sum of Differences } & & & 0.79 & -0.66 & 0.10 \\
\hline
\end{tabular}

Table 7-3. CHEQ output for three LX-14 high pressure adiabats with the $1800 \mathrm{~K}$ freeze. The point at $0.347 \mathrm{Mb}$ is the Principal Adiabat. The CHEQ detonation velocity of $0.897 \mathrm{~cm} / \mu \mathrm{s}$ is slightly higher than the measured $0.878 \mathrm{~cm} / \mu$ s value; CHEQ also gives a detonation pressure of $0.347 \mathrm{Mb}$ whereas our cylinder JWL uses $0.373 \mathrm{Mb}$.

\begin{tabular}{ccccc}
$\begin{array}{c}\text { Detonation } \\
\text { Pressure } \\
\mathrm{P}_{\mathrm{j}}(\mathrm{Mb})\end{array}$ & $\begin{array}{c}\text { Relative } \\
\text { Volume } \\
\mathrm{v}\end{array}$ & $\begin{array}{c}\text { Detonation } \\
\text { Velocity } \\
\mathrm{D}(\mathrm{cm} / \mu \mathrm{s})\end{array}$ & $\omega$ & $\begin{array}{c}\text { Detonation } \\
\text { Energy } \\
E_{\mathrm{o}}(\infty)\end{array}$ \\
\hline 0.347 & 0.764 & 0.897 & 0.326 & 0.106 \\
0.531 & 0.673 & 0.942 & 0.331 & 0.105 \\
0.728 & 0.613 & 1.014 & 0.344 & 0.102 \\
1.000 & 0.558 & 1.111 & 0.350 & 0.098
\end{tabular}

\begin{tabular}{ccccc} 
Cylinder & \multicolumn{4}{c}{ Energy of Detonation $(\mathrm{Mb} \cdot \mathrm{cc} / \mathrm{cc})$} \\
\cline { 2 - 5 } Volume, $\mathrm{v}$ & $0.347 \mathrm{Mb}$ & $0.53 \mathrm{Mb}$ & $0.73 \mathrm{Mb}$ & $1.00 \mathrm{Mb}$ \\
\hline 2.2 & 0.073 & 0.069 & 0.061 & 0.048 \\
4.1 & 0.082 & 0.080 & 0.075 & 0.065 \\
6.5 & 0.086 & 0.084 & 0.080 & 0.071
\end{tabular}


Table 7-4. JWL's for the Prinicipal Adiabat and three high pressure adiabats with the $1800 \mathrm{~K}$ freeze.

\begin{tabular}{ccccc} 
& Principal & $0.53 \mathrm{Mb}$ & $0.73 \mathrm{Mb}$ & $1.00 \mathrm{Mb}$ \\
\cline { 2 - 5 }$\rho_{\mathrm{o}}$ & 1.825 & 1.825 & 1.825 & 1.825 \\
$\mathrm{~A}$ & 7.65434 & 10.87615 & 14.93062 & 20.49784 \\
$\mathrm{~B}$ & 0.21932 & 0.31164 & 0.42781 & 0.58733 \\
$\mathrm{C}$ & 0.01330 & 0.01428 & 0.01640 & 0.02052 \\
$\mathrm{R}_{1}$ & 4.5 & 4.932 & 5.456 & 6.054 \\
$\mathrm{R}_{2}$ & 1.5 & 1.5 & 1.5 & 1.5 \\
$\omega$ & 0.33 & 0.33 & 0.33 & 0.33 \\
$\mathrm{E}_{\mathrm{o}}$ & 0.1060 & 0.1060 & 0.1060 & 0.1060 \\
$\Gamma_{\mathrm{j}}+1$ & 3.787 & 3.754 & 3.712 & 3.667 \\
$\mathrm{D}$ & 0.878 & 0.943 & 1.016 & 1.113 \\
$\mathrm{P}_{\mathrm{h}}$ & 0.373 & 0.531 & 0.728 & 1.000 \\
$\mathrm{Vh}$ & 0.735 & 0.673 & 0.613 & 0.558
\end{tabular}




\section{Chapter 8. Size Effects}

\section{Reaction Zone Definitions}

There are two major size effects in the detonation of a cylinder of explosive. The "forward," ie. the "size" effect, is the reduction of the detonation velocity with the decrease of the radius until detonation finally stops as shown by the list of data in Tables 8-1 and 8-2.1-23 The "transverse" effect is the increase of curvature of the detonation front with decreasing radius as shown by the data assembled in Table 8-3. 14, 20, 24-33 Any successful theory must relate the forward and transverse effects. We here seek to describe the steady state results in a self-consistent way. As usual, the calculation is not from first principles but is calibrated against other data.

The reaction zone length needs to be carefully defined. It is usually defined as $x_{\mathfrak{r}}$, the steady state distance over which an explosive burns all the way to a burn fraction, F, of 1.00 . In Ignition \& Growth (I\&G), it is easier to take the distance as being from the graphical contours $0.1<\mathrm{F}<0.9$. If we have a considerable size effect, so much energy may be lost out the sides of the cylinder that not all of the burn contributes to pushing the detonation front. That part of the zone behind the sonic plane has lost contact with the front. For this case, we define an effective reaction zone length, $\mathrm{x}_{\mathrm{e}}<$ $\mathbf{x}_{\mathbf{r}}$.

From Chapter 1, Eq. 10, we estimate that

$$
\langle\mathrm{F}\rangle \approx \frac{\left\langle\mathrm{E}_{\mathrm{o}}\right\rangle}{\mathrm{E}_{\mathrm{o}}} \approx\left(\frac{\mathrm{U}_{\mathrm{s}}}{\mathrm{D}}\right)^{2}
$$

where $E_{0}$ and $D$ are the total detonation energy and detonation velocity with no size effect; $\left.<E_{0}\right\rangle,<$ $F>$ and $U_{S}$ the average detonation energy, average burn fraction and actual detonation velocity with the size effect. Thus, $<E_{0}>$ becomes defined as energy that affects the forward direction of the detonation front.

\section{Size Effect on Detonation Velocity}

It is well known that the "true" steady state detonation velocity, $\mathrm{D}$, has to be defined for a large sample. If a cylindrical sample is used, the detonation velocity, $U_{S}$, decreases as the radius shrinks until finally, at the failure radius, $R_{f}$, the detonation does not propagate. The wall moves 
out and the explosive detonates at a lower pressure. The explosive near the wall does not burn all the way and cannot sustain the steady state detonation velocity. Energy flows transversely across the detonation front to make up the difference and the entire front is brought to a constant speed, but with a curvature. Finally, the radius becomes so small that the energy cannot get to the edge fast enough and the detonation fizzles.

Table 8-1 shows a variety of size data. The failure radius is the smallest radius for which the cylinder, usually unconfined on the sides, detonated in the opinion of the researcher. In some cases, an actual failure was not reached so that the "failure" radius could be pushed lower. Usually one length of cylinder was run so that the effect of cylinder length is unknown. The smallest measured radius for which detonation propagates is here called the failure radius, for which the detonation velocity is $\mathrm{U}_{\mathbf{s f}}$. The order of the data in Table 8-1 is by increasing reaction zone length, which is calculated in a later section.

Campbell and Engleke fitted a large number of explosives to the form ${ }^{34}$

$$
\frac{U_{s}}{D}=1-\frac{A}{R-R_{c}}
$$

where $R$ is the radius and $A$ and $R_{c}$ are constants. The constant $R_{c}$ was probably intended to represent $R_{f}$, but the fitted results do not agree. The constant $A$ is proportional to the expected reaction zone length but appears smaller by a factor of 10 to 25 for HMX to TATB explosives. The results from Eq. 2 suggest that we should not try to include the failure radius in the equation.

The variation in $U_{s f} f$, shown in Table 8-1, shows how much the explosive can give up and still function. It barely changes for nitromethane but swings over a huge range for ANFO. It is tempting to suggest that the ratio is small for homogenous explosives or those with abundant oxygen and large for inhomogeneous explosives with low oxygen content. However, the following have $U_{s f} f D$ of 0.97 or greater: Comp A, liquid TNT, NM, octol, PBX-9502 and other TATB formulations and XTX-8003. This group has a wide assortment of compositions. It appears that a thermochemical code will be needed to work out the reasons for the observed failure radii.

Eyring, et. al. suggested the Inverse Radius model, which is Eq. 2 without the failure radius. ${ }^{35}$ The underlying reason for this model is as follows. For a bare explosive cylinder, consider a layer near the edge with a thickness, $R_{e}$. All energy in this layer gets sucked out of the sample by the blow-out at the edge and cannot contribute to the forward-moving detonation velocity. All the 
chemical energy $E_{0}$ is available from the rest of the sample. Some of it is shipped to the edge, and the entire sample is averaged to a lower overall value $\left\langle\mathrm{E}_{0}\right\rangle$. We have

$$
\frac{\left.<\mathrm{E}_{\mathrm{o}}\right\rangle}{\mathrm{E}_{\mathrm{o}}}=\left(\frac{\mathrm{R}_{\mathrm{o}}-\mathrm{R}_{\mathrm{e}}}{\mathrm{R}_{\mathrm{o}}}\right)^{2}
$$

Now, we set $\left(E_{a} / E_{0}\right) \approx\left(U_{s} D\right)^{2}$ from $E q$. 1. To further show that this is reasonable, we consider data for eight densities of HNS between 1.00 and $1.68 \mathrm{~g} / \mathrm{cc}^{36}$ The ratio $\mathrm{D}^{2} / \mathrm{E}_{\mathrm{o}}$ is $6.9 \pm 0.2$ or $\pm 3 \%$ for the set.

We convert Eq. 3 to get

$$
\frac{U_{s}}{D}=1-\frac{R_{e}}{R_{o}} \text {. }
$$

We have mentioned that $R_{e}$ is not the reaction zone. From the plate shots of Chapter 6 , we found these approximate $\mathrm{x}_{\mathrm{e}}$ values: PETN $<0.10 \mathrm{~mm}$, HMX (from LX-14) $<0.26 \mathrm{~mm}$ and TATB (from LX17) $>2.5 \mathrm{~mm}$. The rate stick data gives $R_{e}$ values of $0.0008,0.02$ to 0.1 and $0.2 \mathrm{~mm}$ for PETN, HMX and TATB, respectively. The difference is an order of magnitude. The reason for the difference is that $\mathrm{x}_{e}$ and $\mathrm{R}_{e}$ are different lengths perpendicular to one another. The two must be related through a model of the moving wall. We convert the Inverse Radius model into the form

$$
\frac{\mathrm{U}_{\mathrm{s}}}{\mathrm{D}}=1-\frac{<\mathrm{x}_{\mathrm{e}}>}{\sigma \mathrm{R}_{\mathrm{o}}}
$$

where we now have to calculate the constant $\sigma$. The reaction zone length $\left\langle x_{e}\right\rangle$ is an average across the detonation front, where we might expect $\left\langle x_{e}\right\rangle=x_{e}$ at about $R_{0} / 2$.

\section{Calculating the Inverse Radius Model}

We can imagine three basic types of models for the edge layer model depending of whether the reaction zone is short, intermediate or long. A second distinction is whether the explosive is bare or confined in metal.

1. Zone the width of the Shock Wave, $x_{r}=x_{e} \approx x_{S} \ll R_{0}$. We first consider an unconfined explosive cylinder with the reaction zone being the size of the shock front, $x_{S}$, so that the 
"instant" of impact model holds. In this case, the zone length is probably $\mathrm{x}_{\mathbf{r}}$, the real one. The edge layer, $R_{e}$, is perpendicular to the reaction zone as seen below.

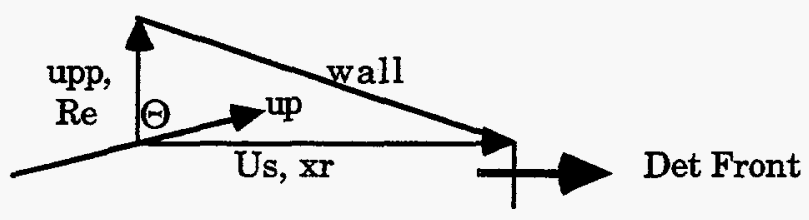

The distances $x_{r}$ and $R_{e}$ are assumed to be proportional to the velocities $U_{s}$ and $u_{p p}$. From the picture above, using $u_{p} / U_{S}=1-v$, we have

$$
R_{e}=[(1-v) \cos \Theta] x_{r}=\frac{x_{r}}{\sigma}
$$

For the unconfined explosive, we estimate that $\Theta \approx 75^{\circ}$ so that $\cos \Theta \approx 1 / 4$. At the C-J point, $(1-v)$ $\approx 1 / 4$, so that $\sigma \approx 16$. If the explosive is confined, we have the problem we had in Chapter 4 . The confinement does not change the angle $\Theta$ but the refraction causes a smaller angle to open up on the inside of the metal wall. This is equivalent to increasing $\Theta$ to perhaps $85^{\circ}$ so that $\sigma \approx 50$. PETN is probably the only explosive we have that really fits in this category.

2. Long Zone. $x_{S} \ll x_{e} \approx R_{0}<x_{r}$. The codes show a rarifaction wave moving inward from the edge. The angle of this wave, $\gamma$, to the original edge is about the same as that of the edge now being pushed, ie. about $15^{\circ}$ for the confined case and $25^{\circ}-30^{\circ}$ for the unconfined. Between the rarifaction wave and the edge, all mass is moving directly outward. The edge layer is calculated from

$$
\tan \gamma=\frac{2 R_{e}}{x_{e}}=\frac{2}{\sigma}
$$

where the "2" comes from the average of the triangular edge blow-out. We calculate $\sigma$ to be 4 for the unconfined case and 7.5 for the confined.

We have to calibrate between the two extreme numbers. We use the plate-measured reaction zones from Chapter 6 as standards: $0.1 \mathrm{~mm}$ for PETN, $0.25 \mathrm{~mm}$ for HMX and $2.5 \mathrm{~mm}$ for TATB. For very long reaction zones, we let $\sigma$ approach 1 . This produces reaction zones of the order of the radius, which is probably the longest they can be. Also, this agrees with the calculated reaction zones done by David Kennedy for HANFO. ${ }^{13}$ The distance that the side rarifaction wave eats in is 
the integral of $\mathrm{Ct}$, where $\mathrm{C}$, the speed of sound, will decrease exponentially as the pressure decreases. We fold all this into the empirical equations

$$
\begin{aligned}
& \sigma(\text { unconfined })=15 \exp \left[-\mathrm{b} \frac{<\mathrm{x}_{\mathrm{e}}>}{\mathrm{x}_{\mathrm{s}}}\right]+1=15 \exp \left(-0.28<\mathrm{x}_{\mathrm{e}}>\right)+1 \\
& \sigma(\text { confined })=49 \exp \left[-\mathrm{b} \frac{<\mathrm{x}_{\mathrm{e}}>}{\mathrm{x}_{\mathrm{s}}}\right]+1=49 \exp \left(-0.28<\mathrm{x}_{\mathrm{e}}>\right)+1
\end{aligned}
$$

The exponential is a function of the ratio of the reaction zone to the shock wave thickness. They are especially useful for HMX explosives, which are almost ideal. For composites, where $\sigma-\rightarrow 1$, the theory approaches the Eyring Inverse Radius in the form that it oftens appears in. Hidden in this is the reduction of $\left\langle x_{e}>\right.$ as one goes from no confinement to confinement.

We now try out the Inverse Radius model where the reaction zone length is

$$
<\mathrm{X}_{e}>=\sigma \mathrm{R}_{\mathrm{o}}\left(1-\frac{\mathrm{U}_{\mathrm{s}}}{\mathrm{D}}\right)
$$

where $\mathrm{D}$ is the infinite-radius velocity for that particular density. The results are listed in Tables 81 and 8-2. The large-radius reaction zone is given as $\left\langle x_{e}(\infty)\right\rangle$. The data for $R_{0}$ and $U_{s}$ listed in Table 8-1 allows recalculation later should the model change. The infinite size detonation velocity $D$

\begin{tabular}{|c|c|c|c|}
\hline & $\begin{array}{c}\mathrm{D} \\
(\mathrm{mm} / \mu \mathrm{s})\end{array}$ & $\begin{array}{c}\left\langle\mathrm{x}_{\mathrm{e}}(\infty)\right\rangle \\
(\mathrm{mm})\end{array}$ & Source \\
\hline \multirow[t]{2}{*}{ ANFO $1.248 \mathrm{~g} / \mathrm{cc}$} & 6.45 & 14 & 1/R extrap. \\
\hline & 6.79 & 14 & Lee \\
\hline \multirow[t]{2}{*}{ HANFO } & 5.35 & 25 & 1/R extrap. \\
\hline & 5.89 & 34 & Kennedy \\
\hline \multirow[t]{3}{*}{ PBXN-111 } & 6.03 & 9 & 1/R extrap. \\
\hline & 6.20 & 13 & Forbes \\
\hline & 7.06 & 20 & CHEETAH \\
\hline
\end{tabular}
is obtained by extrapolating the $U_{S}$ data inversely with the radius. For long reaction zone explosives, this can lead to considerable uncertainty, as seen here:

The high CHEETAH value for PBXN-111 suggests that not all the aluminum is reacting in the cylinder shots. 
Fig. 8-1 shows the basic types of reaction zone vs. radius curves. The short reaction zone explosives with HMX and RDX-Comp B, PBX-9501, Comp A, and PBX-9404 - all show the reaction zone growing larger as the radius decreases. It is not just the short zone, because no change is seen for XTX-8003 and the NM samples. The second basic kind of shape in Fig. 8-1 is shown by the nearly pure TATB, which has almost no change in zone length with radius. This unchangeability of $\left\langle x_{e}>\right.$ with the Inverse Radius model appears in most explosives and especially those with long reaction zones. Octol is unique in showing a large decrease with decreasing radius.

Fig. 8-2 shows the considerable variability for Comp B. ${ }^{3}$ Type 1 consists of $80 \%$ particles less than $400 \mu \mathrm{m}$ while Type 2 is larger, with particles between 417 and $833 \mu \mathrm{m}$. The results show that the larger particles result in a longer reaction zone. Types 3 and 4 appear to be Type 1 with variable cooling rates once the TNT is melted around the RDX. Type 3 has a 5 to 6 hour cool and Type 4 is twice as long. The cooling rate does not affect the basic behavior of the reaction zone.

\section{Detonation Front Curvature Fits}

Another historical approach to the size effect was to use the Wood-Kirkwood equation, ${ }^{37}$ which tries to relate detonation velocity with detonation front curvature. If the curvature is considered spherical, then a radius of curvature near the wall, $S$, may be described. This radius is probably a local one, because the front is not spherical. In any case, if $S \approx R$, and knowing that the reaction zone thickness is present from above, we have

$$
\mathrm{U}_{\mathrm{s}}=\mathrm{D}\left[1-\frac{\mathrm{Bx}_{\mathrm{r}}}{\mathrm{S}}\right]
$$

where B is a constant. The weakness of Eq. 13 is the indefinite nature of the radius of curvature. There is only one detonation velocity but the radius of curvature varies across the front as shown by Forbes, et. al. ${ }^{30-32}$ This ambiguity was discovered long ago, but many attempts were made to forcefit a spherical wavefront even though the ellipse looked better. ${ }^{38,39}$ However, Eq. 13 has led to complex models, which lay "corner-turning" grids onto two-dimensional hydrocodes. ${ }^{40,41}$ These models assume that a total detonation velocity is a function only of the local curvature and that there is a maximum limiting angle the detonation front can make with the wall. As a further problem, the curvature has to be known before these grids can be constructed. 
The detonation front is curved because the edges lag the middle. The available data is summarized in Table 8-3. The measured maximum lags, $\mathrm{L}_{0}$, at the wall are listed, with all the entries being sorted according to increasing $L_{0} / R_{0}$. The wall angle, $\Theta$, is also listed. This is

$$
\Theta=\tan ^{-1}\left(\frac{\Delta \mathrm{R}}{\Delta \mathrm{L}}\right)_{\text {wall }}
$$

as calculated near the wall using the data. We shall consider two fits to the lag data. The first is quadratic with the lag distance, $L$, and the radius, $R$, are in $\mathrm{mm}$ :

$$
\mathrm{L}=\mathrm{AR}^{2}
$$

This is reminiscent of the quadratic velocity distribution for a non-compressible fluid flowing through a smooth pipe with drag at the walls. ${ }^{42}$ If we convert to relative radius units, we have

$$
\frac{L}{R_{0}}=\operatorname{AR}_{\alpha}\left(\frac{R}{R_{0}}\right)^{2}
$$

The angle that the quadratic function makes with the wall is

$$
\Theta(\text { quadratic })=\tan ^{-1}\left(\frac{1}{2 \mathrm{AR}_{\mathrm{o}}}\right)
$$

In Table 8-3, we see that the measured angles are not far from those using the quadratic function, so this simple expression is not bad. Also, we note that both $\Theta$ and $A R_{0}$ fall into the sorting sequence we are using with $\mathrm{L}_{0} / \mathrm{R}_{0}$.

The second fit is elliptical, with the equation

$$
\frac{\mathrm{R}^{2}}{\mathrm{a}^{2}}+\frac{\mathrm{Y}^{2}}{\mathrm{~b}^{2}}=1
$$

where $a$ and $b$ are constants that define the size of the ellipse. The fits for one run of PBX-9502 are shown in Fig. 8-3 and are typical. ${ }^{29}$ The elliptical fit is better and it is a way for the front to bend more sharply to the wall. Let us move the ellipse upward a distance $b$ so that the new origin is on the edge. To the right, we now have the curve looking like the detonation front. Solving for $Y$ we get 


$$
Y=b\left(1-\frac{R^{2}}{a^{2}}\right)^{1 / 2}-b \approx \frac{a}{2 b^{2}} R^{2}
$$

This says that, for small distances off the cylinder axis, we expect the curvature to be quadratic. The fitted constants for the quadratic and elliptical descriptions of the detonation fronts of various explosives are listed in Table 8-3, where the ordering is by the quadratic coefficient. We also list the $x$-coordinate of the positive focus of the ellipse. We note that the detonation intersects the wall long before it reaches the focus. This makes it difficult to physically relate the properties of the ellipse to a zone width. The wall angle for the ellipse is

$$
\Theta(\text { ellipse })=\tan ^{-1}\left\{\frac{a^{2}}{b^{2}}\left[1-\left(\frac{R_{0}}{a}\right)^{2}\right]^{1 / 2}\right\}
$$

From Table 8-3, we see that the quadratic function does not bend enough and the wall angles are too large. The ellipse is generally a better fit.

Another fit is the Bessel function shape 26

$$
L=L_{x}+\operatorname{Mn}\left\{J_{0}\left[N\left(x-x_{0}\right)\right]\right\}
$$

where $M, N$ and $x_{0}$ are constants. The Bessel function is the damped solution for a circular oscillating membrane. It has the same problem as the ellipse, which is that the "membrane" extends far outside the radius of the explosive cylinder so that only the initial slow roll-off part of the function is used. The physical reason for this fit is not obvious.

The wall angles $\Theta$ in Table 8-3 decrease as the ratio $L_{0} / R_{0}$ increases, ie. the more curvature, the smaller the angle. These wall angles come from the curvature of the detonation front and are not the same as the geometric angle of incidence $\alpha$ in Chapter 5. We recall that close examination showed wall angles of $76^{\circ}$ to $78^{\circ}$ for both HMX and TATB explosives. ${ }^{43,44}$ These angles, however, were obtained by trying to measure the angle exactly at the wall without regard for connecting to the curvature of the front. The HMX result came from an obvious glitch close to the wall. This data led Swift to speculate that the $75^{\circ}$ angle might hold for all explosives. ${ }^{43}$ However, the angle $\Theta$ we measure decreases as the relative lag, $L_{0} / R_{0}$, increases. Fig. 8-4 shows this relative lag for the largest and smallest radii of two explosives. The lag increases as the radius decreases. 


\section{Reaction-Zone Model of the Curvature}

The collected detonation lag data is listed in Table 8-3. The variable used for sorting is the relative edge lag, $\mathrm{L}_{0} / \mathrm{R}_{0}$. A reasonable initial assumption is that the lag gets bigger as the effective reaction zone, $x_{e}$, increases. 45 The Inverse Radius model says that there is zero detonation energy in the edge layer. To make up for it, energy flows out from the inside of the cylinder outward.

Because of the cylindrical geometry, the energy flux must go as $R^{2}$. The reason for the curvature is to speed up the transmission of the energy. Energy moving sideways on a detonation front, even with a pressure gradient, would involve mass flow. It is faster to move energy with the wave velocity entering unburned explosive. We can imagine a transverse wave velocity, $U_{t}$, which increases with increases curvature as

$$
\mathrm{U}_{\mathrm{t}} \approx \mathrm{R}_{\mathrm{o}}^{2}
$$

The relation between the radius, lag and angle is

$$
\tan \Theta^{\prime}=\frac{U_{s}}{U_{t}}=\frac{R}{2 L}
$$

where the angle becomes $\Theta$ at the edge. The detonation lag for any radius becomes

$$
\mathrm{L}=\frac{\mathrm{R}}{2} \frac{\mathrm{U}_{\mathrm{t}}}{\mathrm{U}_{\mathrm{s}}} \text {. }
$$

At the edge, we have

$$
\mathrm{L}_{\mathrm{o}}=\left(\frac{\mathrm{R}_{\mathrm{o}}}{2}\right) \frac{\mathrm{U}_{\mathrm{t}}(\max )}{\mathrm{U}_{\mathrm{s}}}
$$

The curvature is caused by the need to send energy to keep the pressure up at the edge. A reaction zone theory may be created by noticing that there is a limit to the size of $U_{t}(\max )$. It is

$$
\left[1-\mathrm{F}_{\mathrm{u}}(\text { edge })\right] \mathrm{D}^{2}=\mathrm{U}_{\mathrm{s}}^{2}+\mathrm{U}_{\mathrm{t}}(\max )^{2}
$$

where $F_{u}$ (edge) is the unburned fraction at the edge. Eq. 26 is based on the energy-velocity-squared relation of Eq. 1. We calculate $U_{t}(\max )$ from $E q .23$ in the form 


$$
\mathrm{U}_{t}(\max )=\frac{\mathrm{U}_{\mathrm{s}}}{\tan [\Theta(\text { quad })]}
$$

There is a range of explosives, where $x_{e}$ is about 1 to $5 \mathrm{~mm}$ where $U_{S}$ is sufficiently different from $D$ to be easily distinquished but where probably all of the explosive is burned. Then, we expect Eq. 26 (with $F_{u}=0$ ) and Eq. 27 to give about the same result.

All of our work in this chapter is based on average values, which occur at $R=R_{d} / 2$. Then $\left.<U_{t}\right\rangle=U_{t}(\max ) / 4$ and the average unburned fraction is

$$
F_{u} \text { (edge) }=1-\frac{1}{D^{2}}\left[U_{s}^{2}+U_{t}(\max )^{2}\right]
$$

In Table 8-3, we use the $\sigma$ values obtained from the Inverse Radius model and calculate $<x_{e}>$ from the measured maximum lags $L_{0}$. We combine Eqs. 5, 24, and 25 to get

$$
\frac{L_{0}}{R_{0}}=\frac{1}{2}\left[\frac{1-F_{u}(\text { edge })}{\left(1-\frac{<x_{e}>}{\sigma R_{0}}\right)^{2}}-1\right]^{1 / 2} \approx\left(\frac{\left.<x_{e}\right\rangle}{2 \sigma R_{0}}\right)^{1 / 2}
$$

The approximate formula at the right holds if $F_{u}$ (edge) $=1$ and $<x_{e}><<\sigma R_{0}$. For a constant reaction zone thickness, $\sigma$ is also constant, so that $I_{0} / R_{0}$ is proportional to $R_{0}{ }^{-1 / 2}$. Eq. 29 predicts that the relative maximum lag will increase as the radius decreases as is seen in practice.

We may solve Eq. 29 for the reaction zone to get

$$
\left\langle x_{e}\right\rangle=\sigma R_{0}\left\{1-\left[\frac{1-F_{u}(\text { edge })}{1+4\left(\frac{L_{0}}{R_{0}}\right)^{2}}\right]^{1 / 2}\right\}
$$

In Table 8-3, we calculate $<\mathrm{x}_{e}>$ from Eq. 29 solved simultaneously with Eqs. 9 and 10 using the measured ratio of $\left(L_{0} / R_{0}\right)$. Using the quadratic ratio (equal to $A R_{0}$ ) would produce slightly larger reaction zones. There is agreement between the reaction zones calculated by the size effect and the maximum detonation lag for the TATB mixtures, the PBNX-111 and the various ANFO's. However, the AP shows a tiny curvature combined with a long reaction zone. The Comp B, octol, and IRX-3A 
all are expected to have small reaction zones but show large curvatures. Admittedly, the data for these explosives was taken separately for the size effect and curvature as it was for the TATB. Only the PBXN-111, the $1.25 \mathrm{~g} / \mathrm{cc}$ ANFO and the HANFO have matched experiments.

It is tempting to try to match the reaction zone with the oxygen balance and then go to the curvature. These three explosives have progressively less oxygen and we might guess their zone lengths as follows.

\begin{tabular}{ccc} 
& \multicolumn{2}{c}{ Reaction Zone, $\mathrm{x}_{\mathrm{r}}(\mathrm{mm})$} \\
\cline { 2 - 3 } PETN & Unconfined & Confined \\
\cline { 2 - 3 } HMX & 0.02 & 0.01 \\
TATB & 0.2 & 0.1 \\
& 2.0 & 2.0
\end{tabular}

The TATB curvature is generally larger than HMX as shown in this scheme, but trying to use Eq. 31 in any quantitative way with binders and mixtures does not work, especially with examples where the reaction zone is between a few tenths of a $\mathrm{mm}$ and $\mathrm{mm}$. It is possible that the properties can be easily changed according to the details of the morphology for these explosives. For zones of several $\mathrm{mm}$ and longer, the agreement is good. Apparently, the disorder reaches a constant level for these composites.

\section{Estimate of the Shadow Velocity}

In a production code, a shadow velocity, $U^{*}$, is used to model corner turning. The greater the curvature, the less will be the shadow velocity in proportion to the bulk velocity $D$.

\begin{tabular}{|c|c|c|c|c|}
\hline & \multicolumn{2}{|c|}{ Det Velocity $(\mathrm{mm} / \mu \mathrm{s})$} & \multirow[b]{2}{*}{ Ratio } & \multirow{2}{*}{$\begin{array}{c}12.7 \mathrm{~mm} \\
\text { meas } \\
\mathrm{L}_{0} / \mathrm{R}_{\mathrm{o}}\end{array}$} \\
\hline & $\begin{array}{c}\text { Main } \\
\text { D }\end{array}$ & $\begin{array}{c}\text { Shadow } \\
U^{*}\end{array}$ & & \\
\hline LX-14 & 0.883 & 0.879 & 0.0045 & \\
\hline $\mathrm{RX}-26-\mathrm{AF}$ & 0.824 & 0.81 & 0.017 & \\
\hline LX-04 & 0.848 & 0.83 & 0.021 & 0.012 \\
\hline LX-20 & 0.806 & 0.78 & 0.032 & \\
\hline RX-08-FO & 0.806 & 0.78 & 0.032 & \\
\hline LX-17 & 0.76 & 0.42 & 0.447 & 0.073 \\
\hline
\end{tabular}


Using the only two data points from $12.7 \mathrm{~mm}$ radius copper cylinders, we have

$$
1-\frac{\mathrm{U}^{*}}{\mathrm{D}}=38\left(\frac{\mathrm{L}_{\mathrm{o}}}{\mathrm{R}_{\mathrm{o}}}\right)^{1.7}
$$

Eq. 33 allows the estimate of a shadow velocity from the measured cylinder curvature.

\section{References}

1. LASL Explosive Property Data, T. R. Gibbs and A. Popolato, ed. (University of California, Berkeley, 1980), pp. 234-249.

2. Y. Kato and C. Brochet, "Cellular Structure of Detonation in Nitromethane containing Aluminum Particles," Proceedings Sixth Symposium (International) on Detonation, Coronado, CA, August 2427, 1976, p. 124.

3. M. E. Malin, A. W. Campbell and C. W. Mautz, "Particle Size Effects in One- and Two-Component Explosives" (Comp B), Second ONR Symposium on Detonation, White Oak, MD, February 11, 1955, p. 478.

4. E. A. Igel and L. B. Seely, Jr., "The Detonation Behavior of Liquid TNT," Second ONR Symposium on Detonation. White Oak, MD, February 11, 1955, p. 439.

5. A. W. Campbell, Propellants, Explosives \& Pyrotechnics $\underline{9} 183$ (1984).

6. W. E. Gordon, "Detonation Limits in Condensed Explosives," Proceedings Fourth Symposium (International) on Detonation, White Oak, MD, October 12-15, 1965, p.179 taken from L. N. Stesik and L. N. Akimova, Russ. J. Phys. Chem., 33 (8) 148 (1959).

7. A. W. Campbell, H. L. Flaugh, A. Popolato and J. B. Ramsey, "Customized Explosives Based on Plastic-Bonded Mixtures to TATB and HMX," Proceedings Seventh Symposium (International) on Detonation, Annapolis, MD. June 16-19, 1981, p. 566.

8. D. Price, A. R. Clairmont, Jr. and J. O. Erkman, Comb. \& Flame 20389 (1973).

9. Clark Bonner, Ireco (Dyno Nobel) Corp., private communication, 1992.

10. W. E. Gordon, "Detonation Limits in Condensed Explosives," Proceedings Fourth Symposium (International) on Detonation. White Oak, MD, October 12-15, 1965, p.179 taken from M. A. Cook, E. B. Mayfield and W. S. Partridge, J. Phys. Chem. 59675 (1955).

11. W. E. Gordon, "Detonation Limits in Condensed Explosives," Proceedings Fourth Symposium (International) on Detonation, White Oak, MD, October 12-15, 1965, p.179 taken from Hino and Yokogawa (not cited).

12. W. E. Gordon, "Detonation Limits in Condensed Explosives," Proceedings Fourth Symposium (International) on Detonation, White Oak, MD, October 12-15, 1965, p.179 taken from W. H. Anderson and R. E. Pesante, Eighth Symposium (International) on Combustion (Williams and Wilkens Co., Baltimore, 1963), p. 705. 
13. J. W. Forbes, E. R. Lemar and R. N. Baker, "Detonation Wave Propagation in PBXW-115," Proceedings Ninth Symposium (International) on Detonation, Portland, OR, August 28- September $\underline{1.1989}$, p. 806.

14. J. W. Forbes, E. R. Lemar, G. T. Sutherland and R. N. Baker, Detonation Wave Curvature, Corner Turning and Unreacted Hugoniot of PBXN-111, Naval Surface Warfare Center Report NSWCDD/TR-92/164, Silver Spring, MD, 1992.

15. Jerry Forbes, Naval Surface Warfare Center, Silver Spring, MD, Private Communication, 1995.

16. M. Finger, F. Helm, E. Lee, R. Boat, H. Cheung, J. Walton, B. Hayes and L. Penn, "Characterization of Commercial Composite Explosives," Proceedings Sixth Symposium (International) on Detonation, Coronado, CA, August 24-27, 1976, p. 729.

17. F. Helm, M. Finger, B. Hayes, E. Lee, H. Cheung and J. Walton, High Explosive Characterization for the Dice Throw Event, LLNL report UCRL-52042 (1976).

18. Jaimin Lee, Detonation Shock Dynamics of Composite Energetic Materials, $\mathrm{PhD}$ Thesis, New Mexico Institute of Mining and Technology, Socorro, New Mexico, 1990. Per-Anders Persson kindly lent a copy of the thesis.

19. W. E. Gordon, "Detonation Limits in Condensed Explosives," Proceedings Fourth Symposium (International) on Detonation, White Oak, MD, October 12-15, 1965, p.179 taken from M. A. Cook and W. S. Partridge, J. Chem. Phys. 59673 (1955).

20. David Kennedy, ICI Australia Operations, Kurri Kurri, New South Wales, Australia, private communication, 1995.

21. Don Craney, Dyno Nobel Corp., private communication, 1993.

22. P. C. Souers, D. B. Larson and C. M. Tarver, Performance Calculations on the ANFO Explosive RX-HD, LLNL Report UCRL-ID-118969 (1995).

23. M. L. Pandow, K. F. Ockert and H. M. Shuey, "Studies of the Diameter-Dependence of Detonation Velocity in Solid Composite Propellants," Proceedings Fourth Symposium (International) on Detonation, White Oak, MD, October 12-15, 1965, p. 96.

24. Copper LLNL cylinder shot data.

25. J. O. Erkman and D. Price, Comparison of Curvature of the Detonation Front in AP with that found in some Conventional Explosives, U.S. Naval Ordnance Laboratory, White Oak, MD, Report NOLTR 69-235 (1970).

26. F. Chaisse' and J. N. Oeconomos, "The Shape Analysis of a Steady Detonation Front in Right Circular Cylinders of High Density Explosive. Some Theoretical and Numerical Aspects," Proceedings Tenth Symposium (International) on Detonation, Boston, MA, July 12-16, 1993, p. 50.

27. E. R. Lemar and J. W. Forbes, "Detonation Wave Curvature of Cast Comp B and PBXN-111," High-Pressure Science and Technology-1993, Proceedings American Physical Society Topical Group on Shock Compression, Colorado Springs, CO. June 28-July 2, 1993, part 2, p. 1385.

28. G. T. Sutherland, E. R. Lamar, J. W. Forbes, E. Anderson, P. Miller, K. D. Ashwell, R. N. Baker, and T. P. Liddiard, "Shock Wave and Detonation Wave Response of Selected HMX Based Research 
Explosives with HTPB Binder Systems," (IRX-1 and IRX-3A), High-Pressure Science and Technology-1993. Proceedings American Physical Society Topical Group on Shock Compression. Colorado Springs, CO. June 28-July 2, 1993, part 2, p. 1413.

29. John Bdzil, Los Alamos Scientific Laboratory, private communication, 1993.

30. E. R. Lemar, J. W. Forbes and G. T. Sutherland, "Detonation Wave Velocity and Curvature of IRX-4 and PBXN-110," Proceedings American Physical Society Topical Conference on Shock Compression of Condensed Matter-1995, Seattle, WA, August 13-18, 1995.

31. J. W. Forbes and E. R. Lemar, "Detonation Wave Velocity and Curvature of Brass Encased PBXN-111," Proceedings American Physical Society Topical Conference on Shock Compression of Condensed Matter- 1995, Seattle, WA, August 13-18, 1995.

32. J. W. Forbes, E. R. Lemar and R. N. Baker, "Detonation Wave Curvature of PBXN-111," HighPressure Science and Technology-1993. Proceedings American Physical Society Topical Group on Shock Compression. Colorado Springs, CO. June 28-July 2, 1993, part 2, p. 1389.

33. W. H. Wilson, J. W. Forbes, P. K. Gustavson, E. R. Lemar and G. T. Sutherland, "Detonation Properties of the Non-Ideal Explosive PBXW-123," Proceedings American Physical Society Topical Conference on Shock Compression of Condensed Matter- 1995. Seattle, WA. August 13-18, 1995.

34. A. W. Campbell and R. Engelke, "The Diameter Effect in High-Density Heterogeneous Explosives," Proceedings Sixth Symposium (International) on Detonation, Coronado, CA, August 24 $-27,1976$, p. 642 .

35. H. Eyring, R. E. Powell, G. H. Duffey and R. B. Parlin, Chem. Rev. 4569 (1949).

36. P. C. Souers and L. C. Haselman, Jr., Detonation Equation of State at LLNL, 1993, LLNL Report UCRL-ID-116113 (March, 1994), p. 5-19.

37. W. W. Wood and J. G. Kirkwood, J. Chem. Phys. 221920 (1954).

38. E. A. Igel and L. B. Seely, Jr., "The Detonation Behavior of Liquid TNT," Second ONR Symposium on Detonation, National Academy of Sciences, Washington, D. C. and U. S. Naval Ordnance Laboratory, White Oak, MD, February 9-11, 1955, p. 439.

39. M. A. Cook, "Detonation Wave Fronts in Ideal and Non-Ideal Detonation," Second ONR Symposium on Detonation, National Academy of Sciences, Washington, D.C. and U. S. Naval Ordnance Laboratory, White Oak, MD, February $9-11,1955$, p. 500.

40. B. D. Lambourn and D. C. Swift, "Application of Whitham's Shock Dynamics Theory to the Propagation of Divergent Detonation Waves," Proceedings Ninth Symposium (International) on Detonation, Portland, OR, August 28-September 1, 1989, p. 784.

41. J. Bdzil, DSD Technology: A Detonation Reactive Huygens Code, LANL Report LA-12235-MS (1992).

42. J. W. Daily and D. R. F. Harleman, Fluid Dynamics (Addison-Wesley, Reading, MA, 1966), pp. 117-118.

43. D. C. Swift, AWE, Aldermaston, England, private communication, 1993.

44. Reference 36, p. 7-2. 
45. C. L. Mader, "Two Dimensional Homogeneous and Heterogeneous Detonation Wave Propagation," Proceedings Sixth Symposium (International) on Detonation, Coronado, CA, August 24-27, 1976, p. 405. 


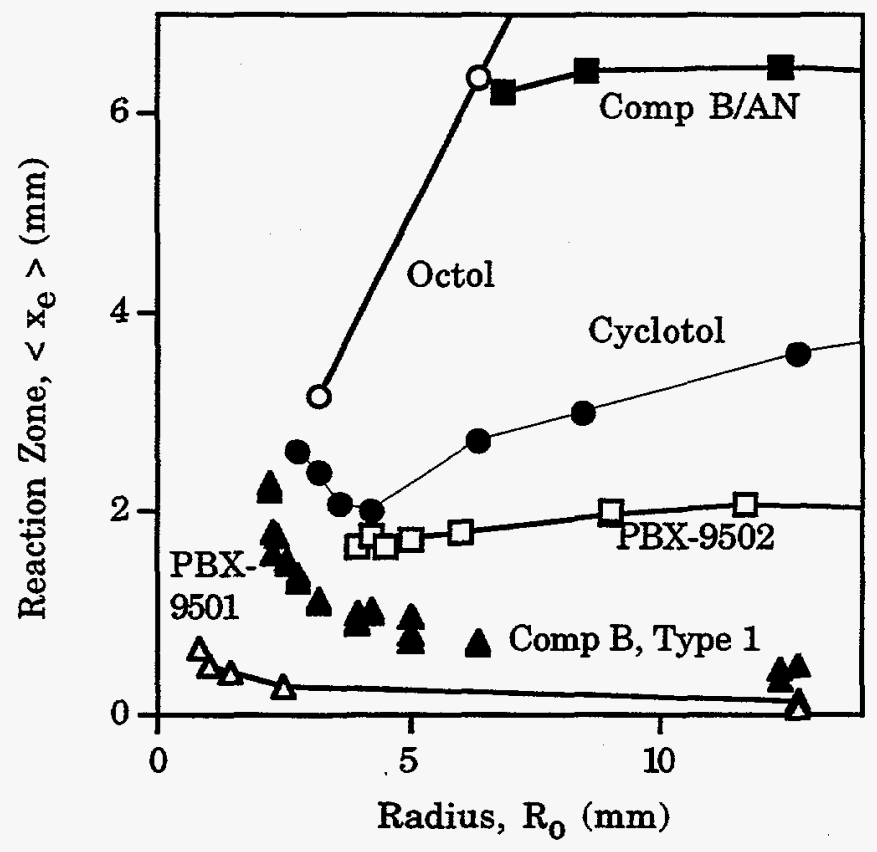

Fig. 8-1. The effective reaction zone length, $x_{e}$, as calculated from rate stick data using the simple Inverse Radius model. RDX and HMX explosives increase with decreasing radius. Many explosives are flat or decrease slightly. Octol is unique in decreasing swiftly.

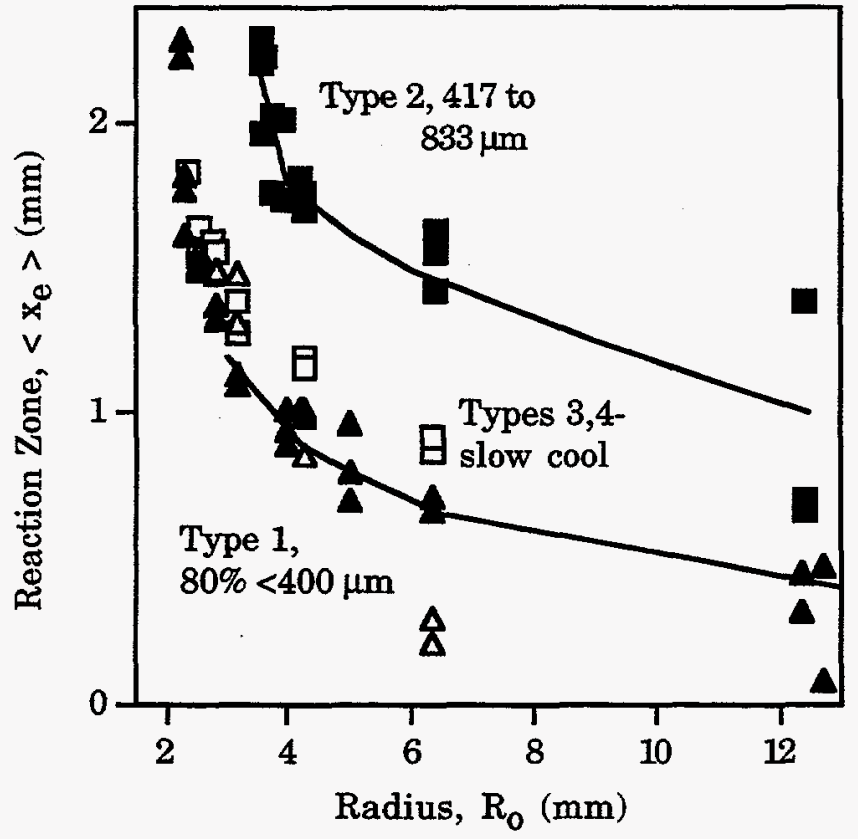

Fig. 8-2. Change of the reaction zone-tadius curve for 4 different blends of Comp B. "Normal" Comp $B$ is type 1 . Types 3 and 4 are type 1 with a slow cool. Big-grained type 2 has a bigger reaction zone than the others. 


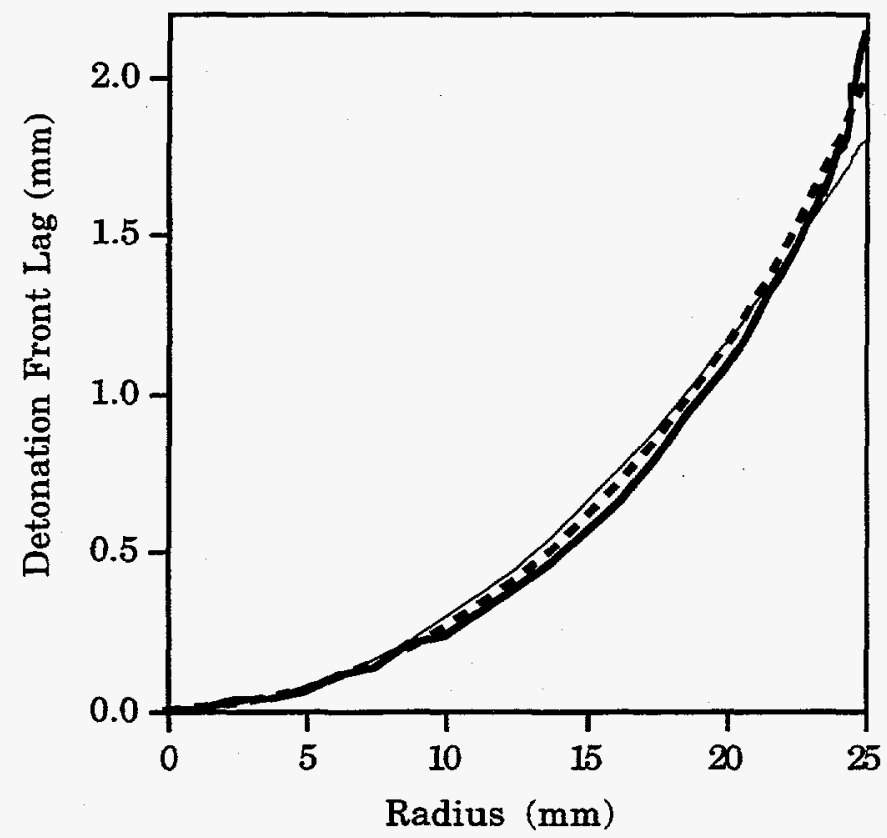

Fig. 8-3. Fitting the detonation velocity lag curve for $25 \mathrm{~mm}$ radius PBX-9502. The data is the heavy line, the quadratic fit is the light line and the ellipse is the dashed line. The ellipse has been moved up a distance $b$ to fit onto the quadratic curve.

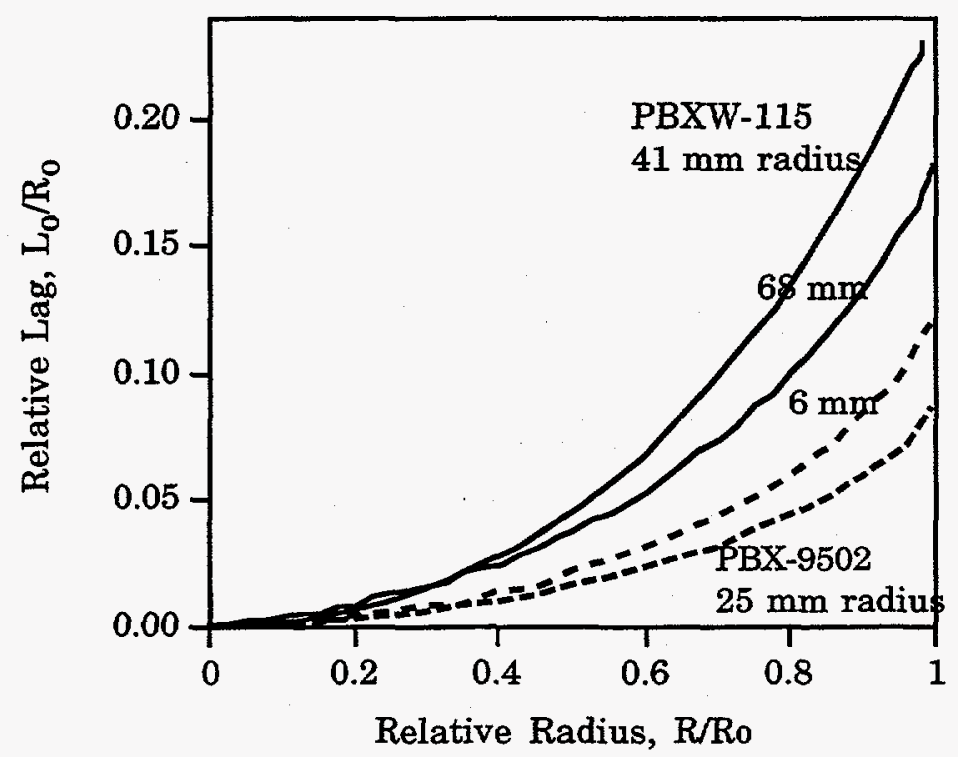

Fig. 8-4. Relative detonation velocity lag for the largest and smallest radii of PBXW-115 and PBX9502. The calculated reaction zone length for PBXW-115 is at least twice that for PBX-9502. It is not known if this affects the amount of relative lag. 
Table 8-1. Size effect and Inverse radius model results for various explosives. The order is from the smallest large-diameter reaction zones to the largest. All rate sticks are unconfined and are at room temperature unless indicated. The symbols are: radius $R_{0}(\mathrm{~mm})$; detonation velocities at $R_{0}, U_{S}$, and for large size, $D,(\mathrm{~mm} / \mu \mathrm{s})$; and the average effective reaction zone $<x_{e}>(\mathrm{mm})$.

\begin{tabular}{|c|c|c|c|c|c|c|c|c|c|c|c|}
\hline $\mathbf{R}_{0}$ & $\mathrm{U}_{\mathrm{s}}$ & $\mathrm{U}_{\mathrm{s}} / \mathrm{D}$ & $\left\langle\mathrm{x}_{\mathrm{e}}\right\rangle$ & $\mathbf{R}_{\mathbf{o}}$ & $\mathrm{U}_{\mathrm{s}}$ & $\mathrm{U}_{\mathbf{s}} \mathrm{D}$ & $\left\langle\mathrm{x}_{\mathrm{e}}\right\rangle$ & $\mathrm{R}_{0}$ & $\mathrm{U}_{\mathrm{s}}$ & $\mathrm{U}_{\mathrm{s}} / \mathrm{D}$ & $\left\langle\mathrm{x}_{\mathrm{e}}\right\rangle$ \\
\hline \multicolumn{2}{|c|}{ XTX-8003 } & & & \multicolumn{3}{|c|}{ Comp A, continued } & & \multicolumn{4}{|c|}{ Comp B Type 1 , continued } \\
\hline large & 7.300 & 1.000 & & 6.35 & 8.254 & 0.998 & 0.19 & 4.24 & 7.725 & 0.981 & 1.0 \\
\hline 0.510 & 7.248 & 0.993 & 0.057 & 4.23 & 8.236 & 0.996 & 0.26 & 4.22 & 7.719 & 0.981 & 1.0 \\
\hline 0.225 & 7.244 & 0.992 & 0.027 & 3.18 & 8.213 & 0.993 & 0.32 & 3.18 & 7.639 & 0.970 & 1.1 \\
\hline 0.130 & 7.167 & 0.982 & 0.038 & 2.54 & 8.172 & 0.988 & 0.43 & 3.17 & 7.650 & 0.972 & 1.1 \\
\hline 0.095 & 7.087 & 0.971 & 0.044 & 2.12 & 8.143 & 0.985 & 0.46 & 2.81 & 7.553 & 0.959 & 1.3 \\
\hline \multicolumn{2}{|c|}{ PBX-9501 } & & & \multicolumn{3}{|c|}{ Comp B, Type 4} & & 2.81 & 7.542 & 0.958 & 1.4 \\
\hline large & 8.795 & 1.000 & & large & 7.9 & & & 2.55 & 7.459 & 0.948 & 1.5 \\
\hline 12.70 & 8.79 & 0.999 & 0.11 & 6.33 & 7.883 & 0.998 & 0.2 & 2.54 & 7.461 & 0.948 & 1.5 \\
\hline 12.70 & 8.791 & 1.000 & 0.09 & 6.33 & 7.875 & 0.997 & 0.3 & 2.33 & 7.317 & 0.929 & 1.8 \\
\hline 12.70 & 8.792 & 1.000 & 0.07 & 4.24 & 7.775 & 0.984 & 0.9 & 2.30 & 7.291 & 0.926 & 1.8 \\
\hline 2.51 & 8.728 & 0.992 & 0.28 & 4.24 & 7.747 & 0.981 & 1.0 & 2.31 & 7.377 & 0.937 & 1.6 \\
\hline 1.42 & 8.612 & 0.979 & 0.42 & 3.18 & 7.562 & 0.957 & 1.5 & 2.22 & 7.088 & 0.900 & 2.2 \\
\hline 1.01 & 8.487 & 0.965 & 0.49 & 3.17 & 7.61 & 0.963 & 1.3 & 2.22 & 7.058 & 0.897 & 2.3 \\
\hline 0.79 & 8.259 & 0.939 & 0.65 & 2.83 & 7.511 & 0.951 & 1.5 & 2.13 & 6.703 & 0.851 & 2.9 \\
\hline \multicolumn{3}{|c|}{ NM, 10\% Al, PMMA } & & 2.82 & 7.516 & 0.951 & 1.5 & \multicolumn{2}{|l|}{$\mathrm{NM}$} & & \\
\hline large & 6.163 & 1.000 & & 2.55 & 7.444 & 0.942 & 1.6 & large & 6.315 & 1.000 & \\
\hline 1.56 & 6.152 & 0.998 & 0.13 & 2.56 & 7.462 & 0.945 & 1.5 & 1.56 & 6.266 & 0.992 & 0.53 \\
\hline 1.25 & 6.144 & 0.997 & 0.18 & \multicolumn{4}{|c|}{ Liquid TNT, $100 \mathrm{oC}$, unconf. } & 1.25 & 6.255 & 0.990 & 0.52 \\
\hline 0.88 & 6.141 & 0.996 & 0.15 & large & 6.560 & 1.000 & & 0.88 & 6.225 & 0.986 & 0.54 \\
\hline 0.65 & 6.135 & 0.995 & 0.14 & 7.56 & 6.549 & 0.998 & 0.19 & 0.65 & 6.227 & 0.986 & 0.41 \\
\hline 0.55 & 6.134 & 0.995 & 0.12 & 4.75 & 6.534 & 0.996 & 0.28 & 0.55 & 6.241 & 0.988 & 0.30 \\
\hline \multicolumn{3}{|c|}{ NM, 5\% Al, PMMA } & & 3.50 & 6.520 & 0.994 & 0.31 & \multicolumn{2}{|c|}{ PBX-9404 } & & \\
\hline large & 6.220 & 1.000 & & 3.25 & 6.515 & 0.993 & 0.33 & large & 8.805 & 1.000 & \\
\hline 1.56 & 6.208 & 0.998 & 0.14 & \multicolumn{3}{|c|}{ Comp B Type 1} & & 73.00 & 8.8 & 0.999 & 0.6 \\
\hline 1.25 & 6.199 & 0.997 & 0.20 & large & 7.872 & & & 73.00 & 8.803 & 1.000 & 0.2 \\
\hline 0.88 & 6.198 & 0.996 & 0.15 & 12.74 & 7.851 & 0.997 & 0.48 & 19.05 & 8.789 & 0.998 & 0.5 \\
\hline 0.65 & 6.188 & 0.995 & 0.16 & 12.72 & 7.869 & 1.000 & 0.08 & 12.70 & 8.774 & 0.996 & 0.6 \\
\hline 0.55 & 6.194 & 0.996 & 0.11 & 12.38 & 7.858 & 0.998 & 0.32 & 12.70 & 8.775 & 0.997 & 0.6 \\
\hline \multicolumn{3}{|c|}{$\mathrm{NM}, 15 \% \mathrm{Al}, \mathrm{PMMA}$} & & 12.38 & 7.858 & 0.998 & 0.32 & 11.45 & 8.793 & 0.999 & 0.2 \\
\hline large & 6.104 & 1.000 & & 12.38 & 7.852 & 0.997 & 0.45 & 8.16 & 8.789 & 0.998 & 0.2 \\
\hline 1.56 & 6.092 & 0.998 & 0.15 & 6.33 & 7.805 & 0.991 & 0.72 & 6.35 & 8.776 & 0.997 & 0.3 \\
\hline 1.25 & 6.092 & 0.998 & 0.12 & 6.33 & 7.810 & 0.992 & 0.67 & 3.19 & 8.731 & 0.992 & 0.4 \\
\hline 0.88 & 6.082 & 0.996 & 0.15 & 5.03 & 7.777 & 0.988 & 0.79 & 1.44 & 8.651 & 0.983 & 0.4 \\
\hline 0.65 & 6.082 & 0.996 & 0.11 & 5.00 & 7.789 & 0.989 & 0.71 & 1.40 & 8.668 & 0.984 & 0.3 \\
\hline 0.55 & 6.076 & 0.995 & 0.12 & 5.00 & 7.751 & 0.985 & 0.97 & 1.00 & 8.525 & 0.968 & 0.5 \\
\hline \multicolumn{2}{|c|}{ Comp A } & & & 3.97 & 7.725 & 0.981 & 0.94 & 0.75 & 8.355 & 0.949 & 0.5 \\
\hline large & 8.270 & 1.000 & & 3.97 & 7.712 & 0.980 & 1.0 & 0.64 & 7.874 & 0.894 & 0.9 \\
\hline 12.69 & 8.262 & 0.999 & 0.19 & 3.97 & 7.733 & 0.982 & 0.9 & 0.61 & 7.279 & 0.827 & 1.2 \\
\hline
\end{tabular}


Table 8-1, part 2

\begin{tabular}{|c|c|c|c|c|c|c|c|c|c|c|c|}
\hline$R_{0}$ & $\mathrm{U}_{\mathrm{s}}$ & $\mathrm{U}_{\mathrm{s}} / \mathrm{D}$ & $\left\langle\mathrm{x}_{\mathrm{e}}\right\rangle$ & $R_{0}$ & $\mathrm{U}_{\mathrm{s}}$ & $\mathrm{U}_{\mathrm{s}} / \mathrm{D}$ & $\left\langle x_{e}\right\rangle$ & $R_{0}$ & $\mathrm{U}_{\mathrm{s}}$ & $\mathrm{U}_{\mathrm{s}} / \mathrm{D}$ & $\left\langle x_{e}\right\rangle$ \\
\hline \multicolumn{4}{|c|}{ Liquid TNT, $100 \circ \mathrm{C}$, confined } & \multicolumn{3}{|c|}{$\mathrm{X}-0219$, continued } & & \multicolumn{4}{|c|}{ TNT, $1.62 \mathrm{~g} / \mathrm{cc}$, continued } \\
\hline large & 6.560 & 1.000 & & 9.00 & 7.397 & 0.977 & 2.0 & 4.20 & 6.940 & 0.980 & 1.0 \\
\hline 7.56 & 6.549 & 0.998 & 0.55 & 7.95 & 7.38 & 0.975 & 2.0 & 3.09 & 6.847 & 0.967 & 1.2 \\
\hline 3.18 & 6.537 & 0.996 & 0.49 & \multicolumn{3}{|c|}{ Comp B, Type 2} & & 2.11 & 6.743 & 0.952 & 1.2 \\
\hline 1.59 & 6.515 & 0.993 & 0.48 & large & 7.820 & 1.000 & & 1.58 & 6.617 & 0.935 & 1.2 \\
\hline \multicolumn{3}{|c|}{ PBX-9502, $75^{\circ} \mathrm{C}$} & & 12.45 & 7.743 & 0.990 & 1.4 & \multicolumn{2}{|l|}{$\mathrm{X}-0344$} & & \\
\hline large & 7.662 & 1.000 & & 12.42 & 7.789 & 0.996 & 0.66 & \begin{tabular}{|l|} 
large \\
\end{tabular} & 8.070 & 1.000 & \\
\hline 25.00 & 7.648 & 0.998 & 0.6 & 12.45 & 7.787 & 0.996 & 0.70 & 20.00 & 8.030 & 0.995 & 1.2 \\
\hline 16.50 & 7.614 & 0.994 & 1.2 & 6.37 & 7.631 & 0.976 & 1.6 & 8.33 & 7.960 & 0.986 & 1.3 \\
\hline 9.00 & 7.548 & 0.985 & 1.5 & 6.37 & 7.643 & 0.977 & 1.6 & 4.54 & 7.880 & 0.976 & 1.3 \\
\hline 6.00 & 7.501 & 0.979 & 1.4 & 6.37 & 7.664 & 0.980 & 1.4 & 4.00 & 7.850 & 0.973 & 1.3 \\
\hline 5.00 & 7.477 & 0.976 & 1.4 & 4.24 & 7.503 & 0.959 & 1.8 & 3.45 & 7.840 & 0.971 & 1.2 \\
\hline 4.00 & 7.439 & 0.971 & 1.3 & 4.24 & 7.516 & 0.961 & 1.7 & 2.94 & 7.810 & 0.968 & 1.1 \\
\hline 3.00 & 7.377 & 0.963 & 1.3 & 4.20 & 7.485 & 0.957 & 1.8 & \multicolumn{3}{|c|}{ TNT, $1.55 \mathrm{~g} / \mathrm{cc}$} & \\
\hline \multicolumn{3}{|c|}{ NM, confined } & & 3.91 & 7.394 & 0.946 & 2.0 & large & 6.905 & 1.000 & \\
\hline 47.63 & 6.210 & 1.000 & & 3.91 & 7.481 & 0.957 & 1.7 & 10.26 & 6.852 & 0.992 & 1.0 \\
\hline 12.70 & 6.201 & 0.999 & 0.75 & 3.75 & 7.372 & 0.943 & 2.0 & 7.74 & 6.787 & 0.983 & 1.5 \\
\hline 12.69 & 6.200 & 0.998 & 0.82 & 3.75 & 7.458 & 0.954 & 1.8 & 5.07 & 6.732 & 0.975 & 1.4 \\
\hline 6.395 & 6.188 & 0.996 & 0.89 & 3.58 & 7.233 & 0.925 & 2.3 & 4.20 & 6.710 & 0.972 & 1.4 \\
\hline 6.350 & 6.190 & 0.997 & 0.82 & 3.60 & 7.268 & 0.929 & 2.2 & 2.61 & 6.589 & 0.954 & 1.4 \\
\hline 3.165 & 6.169 & 0.993 & 0.83 & 3.56 & 7.369 & 0.942 & 2.0 & 1.95 & 6.491 & 0.940 & 1.4 \\
\hline 3.215 & 6.166 & 0.993 & 0.90 & 3.56 & 7.273 & 0.930 & 2.2 & 1.58 & 6.332 & 0.917 & 1.5 \\
\hline 1.525 & 6.125 & 0.986 & 0.83 & \multicolumn{3}{|c|}{ Comp B, Type 3} & & \multicolumn{2}{|l|}{$\mathrm{X}-0343$} & & \\
\hline 1.500 & 6.128 & 0.987 & 0.80 & large & 8.00 & & & large & 7.930 & 1.000 & \\
\hline \multicolumn{2}{|l|}{ Octol } & & & 6.33 & 7.914 & 0.989 & 0.9 & 20.00 & 7.890 & 0.995 & 1.2 \\
\hline large & 8.472 & 1.000 & & 6.33 & 7.908 & 0.989 & 0.9 & 8.33 & 7.800 & 0.984 & 1.5 \\
\hline 25.40 & 8.452 & 0.998 & 0.8 & 4.24 & 7.806 & 0.976 & 1.2 & 4.54 & 7.710 & 0.972 & 1.4 \\
\hline 19.06 & 8.45 & 0.997 & 0.7 & 4.24 & 7.815 & 0.977 & 1.2 & 3.45 & 7.650 & 0.965 & 1.4 \\
\hline 11.45 & 8.415 & 0.993 & 1.0 & 3.17 & 7.717 & 0.965 & 1.3 & 2.94 & 7.600 & 0.958 & 1.4 \\
\hline 11.45 & 8.427 & 0.995 & 0.8 & 3.17 & 7.684 & 0.961 & 1.4 & \multicolumn{2}{|l|}{ Cyclotol } & & \\
\hline 8.15 & 8.402 & 0.992 & 0.9 & 2.79 & 7.566 & 0.946 & 1.6 & 50.80 & 8.290 & 1.000 & \\
\hline 8.15 & 8.4 & 0.992 & 0.9 & 2.83 & 7.583 & 0.948 & 1.6 & 25.40 & 8.217 & 0.991 & 2.1 \\
\hline 6.36 & 8.357 & 0.986 & 1.1 & 2.55 & 7.503 & 0.938 & 1.6 & 12.70 & 8.204 & 0.990 & 1.5 \\
\hline 3.17 & 8.161 & 0.963 & 1.3 & 2.54 & 7.542 & 0.943 & 1.5 & 8.45 & 8.160 & 0.984 & 1.5 \\
\hline \multicolumn{2}{|l|}{$\mathrm{X}-0219$} & & & 2.35 & 7.357 & 0.920 & 1.8 & 6.35 & 8.107 & 0.978 & 1.5 \\
\hline large & 7.573 & 1.000 & & \multicolumn{3}{|c|}{$\mathrm{TNT}, 1.62 \mathrm{~g} / \mathrm{cc}$} & & 4.25 & 8.116 & 0.979 & 1.1 \\
\hline 25.40 & 7.555 & 0.998 & 0.8 & large & 7.080 & 1.000 & & 3.65 & 8.012 & 0.966 & 1.4 \\
\hline 20.60 & 7.531 & 0.994 & 1.3 & 15.76 & 7.000 & 0.989 & 1.7 & 3.20 & 7.859 & 0.948 & 1.7 \\
\hline 12.70 & 7.462 & 0.985 & 1.9 & 11.31 & 7.000 & 0.989 & 1.4 & 2.80 & 7.664 & 0.924 & 2.0 \\
\hline 12.70 & 7.457 & 0.985 & 1.9 & 7.88 & 6.989 & 0.987 & 1.2 & & & & \\
\hline 12.70 & 7.453 & 0.984 & 2.0 & 5.25 & 6.989 & 0.987 & 0.9 & & & & \\
\hline
\end{tabular}


Table 8-1, part 3

\begin{tabular}{|c|c|c|c|c|c|c|c|c|c|c|c|}
\hline $\mathbf{R}_{0}$ & $\mathrm{U}_{\mathrm{s}}$ & $\mathrm{U}_{\mathrm{s}} / \mathrm{D}$ & $\left\langle\mathbf{x}_{\mathrm{e}}\right\rangle$ & $\mathbf{R}_{0}$ & $U_{s}$ & $\mathrm{U}_{\mathrm{s}} / \mathrm{D}$ & $\left\langle\mathrm{x}_{\mathrm{e}}\right\rangle$ & $\mathrm{R}_{\mathrm{o}}$ & $\mathrm{U}_{\mathrm{s}}$ & $\mathrm{U}_{\mathrm{S}} / \mathrm{D}$ & $\left\langle\mathrm{x}_{e}\right\rangle$ \\
\hline \multicolumn{2}{|l|}{$\mathrm{X}-0342$} & & & $\mathrm{X}-0341$ & & & & \multicolumn{2}{|l|}{ QM-100 } & & \\
\hline large & 7.880 & 1.000 & & large & 7.830 & 1.000 & & large & 6.25 & & \\
\hline 20.00 & 7.820 & 0.992 & 1.6 & 20.00 & 7.750 & 0.990 & 2.0 & 152.40 & 6.2 & 0.992 & 6 \\
\hline 8.33 & 7.720 & 0.980 & 1.8 & 8.33 & 7.640 & 0.976 & 2.0 & 76.20 & 6.195 & 0.991 & 5 \\
\hline 4.54 & 7.600 & 0.964 & 1.7 & 4.54 & 7.480 & 0.955 & 2.0 & 50.80 & 5.805 & 0.929 & 4 \\
\hline 4.00 & 7.570 & 0.961 & 1.7 & 4.00 & 7.420 & 0.948 & 2.0 & 38.10 & 5.52 & 0.883 & 5 \\
\hline 3.45 & 7.500 & 0.952 & 1.7 & X-0309 & & & & 31.75 & 5.405 & 0.865 & 4 \\
\hline \multicolumn{3}{|c|}{ PBX-9502, room temp } & & large & 6.774 & 1.000 & & 25.40 & 5.09 & 0.814 & 5 \\
\hline 54.00 & 7.729 & 1.000 & & 50.80 & 6.743 & 0.995 & 2.2 & 19.05 & 3.26 & 0.522 & 10 \\
\hline 25.00 & 7.664 & 0.992 & 2.0 & 50.80 & 6.737 & 0.995 & 2.4 & \multicolumn{2}{|c|}{ TNT, $0.8 \mathrm{~g} / \mathrm{cc}$} & & \\
\hline 25.00 & 7.667 & 0.992 & 2.0 & 38.10 & 6.698 & 0.989 & 3.0 & large & 4.310 & 1.000 & \\
\hline 15.75 & 7.624 & 0.986 & 2.1 & 25.40 & 6.653 & 0.982 & 3.0 & 15.49 & 4.192 & 0.973 & 3.8 \\
\hline 11.65 & 7.586 & 0.981 & 2.1 & 25.40 & 6.654 & 0.982 & 3.0 & 10.43 & 3.841 & 0.891 & 6.1 \\
\hline 9.00 & 7.557 & 0.978 & 2.0 & 25.40 & 6.671 & 0.985 & 2.9 & 7.11 & 3.379 & 0.784 & 5.5 \\
\hline 9.00 & 7.552 & 0.977 & 2.0 & 15.88 & 6.560 & 0.968 & 3.1 & 5.03 & 2.830 & 0.657 & 5.1 \\
\hline 6.00 & 7.503 & 0.971 & 1.8 & \multicolumn{2}{|c|}{ TNT, $1.0 \mathrm{~g} / \mathrm{cc}$} & & & 4.52 & 2.192 & 0.509 & 4.0 \\
\hline 5.00 & 7.475 & 0.967 & 1.7 & large & 4.860 & 1.000 & & 4.03 & 1.863 & 0.432 & 3.9 \\
\hline 4.50 & 7.456 & 0.965 & 1.7 & 15.71 & 4.742 & 0.976 & 2.9 & \multicolumn{3}{|c|}{ AP/Al, $0.62 \mathrm{~g} / \mathrm{cc}$} & \\
\hline 4.25 & 7.42 & 0.960 & 1.8 & 10.43 & 4.555 & 0.937 & 3.1 & large & 3.050 & 1.000 & \\
\hline 4.00 & 7.421 & 0.960 & 1.7 & 7.64 & 4.203 & 0.865 & 2.4 & 38.10 & 2.940 & 0.964 & 6 \\
\hline \multicolumn{3}{|c|}{ PBX-9502, -55oC } & & 5.05 & 3.940 & 0.811 & 2.6 & 25.40 & 2.880 & 0.944 & 6 \\
\hline large & 7.73 & 1.000 & & 4.52 & 3.423 & 0.704 & 2.1 & 17.45 & 2.810 & 0.921 & 6 \\
\hline 25.00 & 7.67 & 0.992 & 1.9 & \multicolumn{2}{|c|}{ AP/Al $0.90 \mathrm{~g} / \mathrm{cc}$} & & & 12.70 & 2.740 & 0.898 & 6 \\
\hline 25.00 & 7.665 & 0.992 & 2.1 & \begin{tabular}{|l} 
large \\
\end{tabular} & 3.940 & 1.000 & & 12.70 & 2.700 & 0.885 & 5 \\
\hline 16.50 & 7.616 & 0.985 & 2.3 & 38.10 & 3.790 & 0.962 & 6 & \multicolumn{4}{|c|}{ Comp B/AN $71 / 29$ cast $1.59 \mathrm{~g} / \mathrm{cc}$} \\
\hline 9.00 & 7.546 & 0.976 & 2.1 & 25.40 & 3.540 & 0.898 & 4 & large & 7.700 & 1.000 & \\
\hline 7.15 & 7.485 & 0.968 & 2.2 & 25.40 & 3.640 & 0.924 & 5 & 69.87 & 7.518 & 0.976 & 6 \\
\hline 6.00 & 7.465 & 0.966 & 2.0 & 12.70 & 3.330 & 0.845 & 4 & 51.23 & 7.551 & 0.981 & 7 \\
\hline 5.50 & 7.424 & 0.960 & 2.1 & \multicolumn{3}{|c|}{$\mathrm{AP} / \mathrm{Al}, 1.10 \mathrm{~g} / \mathrm{cc}$} & & 38.43 & 7.518 & 0.976 & 6 \\
\hline 5.25 & 7.398 & 0.957 & 2.1 & large & 4.400 & 1.000 & & 25.91 & 7.518 & 0.976 & 5 \\
\hline \multicolumn{3}{|c|}{ TNT, $1.46 \mathrm{~g} / \mathrm{cc}$} & & 38.10 & 4.230 & 0.961 & 6 & 19.05 & 7.335 & 0.953 & 6 \\
\hline large & 6.590 & 1.000 & & 38.10 & 4.160 & 0.945 & 4 & 12.40 & 7.103 & 0.923 & 6 \\
\hline 22.06 & 6.551 & 0.994 & 1.5 & 25.40 & 4.150 & 0.943 & 6 & 8.54 & 6.855 & 0.890 & 6 \\
\hline 16.34 & 6.491 & 0.985 & 2.3 & 25.40 & 4.070 & 0.925 & 5 & 6.82 & 6.722 & 0.873 & 6 \\
\hline 10.26 & 6.535 & 0.992 & 1.1 & 17.45 & 3.980 & 0.905 & 5 & \multicolumn{3}{|c|}{ ANFO, $0.95 \mathrm{~g} / \mathrm{cc}$} & \\
\hline 7.88 & 6.447 & 0.978 & 1.8 & 17.45 & 3.930 & 0.893 & 5 & large & 4.720 & 1.000 & \\
\hline 5.25 & 6.387 & 0.969 & 1.7 & 12.70 & 3.780 & 0.859 & 5 & 49.21 & 4.433 & 0.939 & 8.2 \\
\hline 4.20 & 6.338 & 0.962 & 1.7 & 12.70 & 3.800 & 0.864 & 5 & 42.79 & 4.392 & 0.930 & 8.3 \\
\hline 3.13 & 6.108 & 0.927 & 2.2 & 12.70 & 3.800 & 0.864 & 5 & 44.24 & 4.269 & 0.904 & 6.0 \\
\hline 2.61 & 5.943 & 0.902 & 2.4 & & & & & 39.37 & 4.269 & 0.904 & 6.5 \\
\hline 2.37 & 5.757 & 0.874 & 2.6 & & & & & 36.79 & 4.296 & 0.910 & 7.4 \\
\hline
\end{tabular}


Table 8-1, part 4

\begin{tabular}{|c|c|c|c|c|c|c|c|c|c|c|c|}
\hline \multicolumn{4}{|c|}{ Table 8-1, part 4} & \multirow[b]{2}{*}{$R_{0}$} & \multirow[b]{2}{*}{$\mathrm{U}_{\mathrm{s}}$} & \multirow[b]{2}{*}{$\mathrm{U}_{\mathrm{s}} \mathrm{D}$} & \multirow[b]{2}{*}{$\left\langle\mathrm{x}_{\mathrm{e}}\right\rangle$} & & & & \\
\hline $\mathrm{R}_{\mathrm{o}}$ & $\mathrm{U}_{\mathrm{s}}$ & $\mathrm{U}_{\mathrm{s}} / \mathrm{D}$ & $\left\langle\mathrm{x}_{\mathrm{e}}\right\rangle$ & & & & & $\mathrm{R}_{0}$ & $\mathrm{U}_{\mathrm{s}}$ & $\mathrm{U}_{\mathrm{s}} / \mathrm{D}$ & $\left\langle\mathrm{x}_{\mathrm{e}}\right\rangle$ \\
\hline \multicolumn{4}{|c|}{ ANFO, $0.95 \mathrm{~g} / \mathrm{cc}$, continued } & 37.80 & 3.936 & 0.895 & 8 & \multicolumn{3}{|c|}{ HANFO, $1.069 \mathrm{~g} / \mathrm{cc}$} & \\
\hline 35.15 & 4.159 & 0.881 & 6.0 & 32.02 & 3.803 & 0.864 & 8 & large & 5.350 & 1.000 & \\
\hline 31.25 & 4.063 & 0.861 & 5.9 & 25.62 & 3.784 & 0.860 & 9 & 126.85 & 4.302 & 0.804 & 25 \\
\hline 30.05 & 4.132 & 0.875 & 6.5 & 19.05 & 3.661 & 0.832 & 10 & 92.40 & 3.732 & 0.698 & 28 \\
\hline 26.60 & 4.077 & 0.864 & 6.7 & 12.53 & 2.998 & 0.681 & 8 & 92.40 & 3.512 & 0.656 & 32 \\
\hline 24.01 & 3.967 & 0.840 & 6.4 & 9.49 & 2.866 & 0.651 & 10 & 76.35 & 3.277 & 0.613 & 30 \\
\hline 21.17 & 3.844 & 0.814 & 6.2 & 8.01 & 2.354 & 0.535 & 9 & 60.05 & 2.904 & 0.543 & 28 \\
\hline 19.11 & 3.762 & 0.797 & 6.3 & 6.39 & 1.815 & 0.412 & 9 & 50.40 & 2.581 & 0.482 & 26 \\
\hline 16.40 & 3.707 & 0.785 & 6.9 & \multicolumn{3}{|c|}{$\mathrm{AP}, 1.24 \mathrm{~g} / \mathrm{cc}$} & & 50.40 & 2.657 & 0.497 & 26 \\
\hline 14.47 & 3.474 & 0.736 & 6.4 & large & 4.278 & & & 44.85 & 2.364 & 0.442 & 25 \\
\hline 13.21 & 3.406 & 0.722 & 6.6 & 12.70 & 2.650 & 0.619 & 10 & 40.15 & 2.386 & 0.446 & 23 \\
\hline 12.15 & 3.241 & 0.687 & 6.4 & 31.75 & 3.843 & 0.898 & 14 & 35.10 & 2.288 & 0.428 & 21 \\
\hline \multicolumn{3}{|c|}{$\mathrm{AP} / \mathrm{Al}, 1.36 \mathrm{~g} / \mathrm{cc}$} & & \multicolumn{3}{|c|}{$\mathrm{ANFO}, 0.8 \mathrm{~g} / \mathrm{cc}$} & & 35.10 & 2.164 & 0.404 & 22 \\
\hline large & 5.310 & 1.000 & & large & 4.74 & & & 30.30 & 1.917 & 0.358 & 20 \\
\hline 38.10 & 4.910 & 0.925 & 7 & 146.00 & 4.56 & 0.962 & 11 & 29.00 & 1.932 & 0.361 & 20 \\
\hline 25.40 & 4.700 & 0.885 & 7 & 146.00 & 4.55 & 0.960 & 10 & \multicolumn{2}{|c|}{$\mathrm{RX}-\mathrm{HD}$} & & \\
\hline 17.45 & 4.460 & 0.840 & 7 & 51.00 & 3.89 & 0.821 & 11 & large & 6.9 & 1.000 & \\
\hline 12.70 & 4.120 & 0.776 & 7 & 25.50 & 3.25 & 0.686 & 10 & 84.38 & 5.2 & 0.754 & 22 \\
\hline 12.70 & 4.100 & 0.772 & 7 & \multicolumn{3}{|c|}{ ANFO, $1.248 \mathrm{~g} / \mathrm{cc}$} & & 84.38 & 4 & 0.580 & 35 \\
\hline 12.70 & 4.100 & 0.772 & 7 & large & 6.450 & 1.000 & & 62.50 & 4.4 & 0.638 & 23 \\
\hline \multicolumn{3}{|c|}{$\mathrm{AP} 13 \mu \mathrm{m}, 1.0 \mathrm{~g} / \mathrm{cc}$} & & 101.35 & 6.156 & 0.954 & 10 & & & & \\
\hline large & 3.660 & 1.000 & & 51.15 & 5.835 & 0.905 & 10 & & & & \\
\hline 55.45 & 3.337 & 0.912 & 10 & 38.95 & 5.575 & 0.864 & 9 & & & & \\
\hline 34.54 & 3.049 & 0.833 & 8 & 26.25 & 5.275 & 0.818 & 10 & & & & \\
\hline 24.01 & 2.816 & 0.770 & 9 & 20.45 & 4.862 & 0.754 & 9 & & & & \\
\hline 17.12 & 2.282 & 0.624 & 8 & 15.95 & 4.044 & 0.627 & 8 & & & & \\
\hline \multicolumn{2}{|c|}{ PBXN-111 } & & & \multicolumn{4}{|c|}{ TNT/AN, 50/50, $1.53 \mathrm{~g} / \mathrm{cc}$} & & & & \\
\hline large & 6.03 & & & large & 7.050 & 1.000 & & & & & \\
\hline 49.95 & 5.63 & 0.934 & 8 & 69.87 & 6.276 & 0.890 & 12 & & & & \\
\hline 34.55 & 5.54 & 0.919 & 10 & 39.08 & 6.033 & 0.856 & 15 & & & & \\
\hline 24.95 & 5.315 & 0.881 & 9 & 25.06 & 5.426 & 0.770 & 15 & & & & \\
\hline 24.85 & 5.331 & 0.884 & 9 & 18.74 & 4.880 & 0.692 & 15 & & & & \\
\hline 24.80 & 5.365 & 0.890 & 10 & \multicolumn{3}{|c|}{$\mathrm{DNT}, 0.95 \mathrm{~g} / \mathrm{cc}$} & & & & & \\
\hline 24.80 & 5.421 & 0.899 & 11 & large & 4.210 & 1.000 & & & & & \\
\hline 22.25 & 5.228 & 0.867 & 9 & 104.80 & 3.694 & 0.877 & 15 & & & & \\
\hline 20.55 & 5.19 & 0.861 & 9 & 82.34 & 3.558 & 0.845 & 15 & & & & \\
\hline 19.35 & 5.036 & 0.835 & 9 & 64.04 & 3.476 & 0.826 & 15 & & & & \\
\hline \multicolumn{4}{|c|}{ TNT/AN 50/50, $1.00 \mathrm{~g} / \mathrm{cc}$} & 53.62 & 3.123 & 0.742 & 16 & & & & \\
\hline large & 4.400 & 1.000 & & 31.16 & 2.742 & 0.651 & 15 & & & & \\
\hline 51.23 & 4.059 & 0.923 & 8 & 25.06 & 2.389 & 0.567 & 15 & & & & \\
\hline 46.11 & 3.926 & 0.892 & 7 & & & & & & & & \\
\hline
\end{tabular}


Table 8-2. List of size-effect data for cylinders of explosive, mostly unconfined. The Inverse Radius model is used to obtain and order the effective reaction zone length for a large radius, $\left\langle\mathbf{x}_{\mathbf{e}}(\infty)\right\rangle$.

\begin{tabular}{|c|c|c|c|c|c|c|c|c|c|}
\hline \multirow[b]{2}{*}{ Explosive } & \multirow[b]{2}{*}{$\begin{array}{c}\text { Den- } \\
\text { sity } \\
(\mathrm{g} / \mathrm{cc})\end{array}$} & \multirow[b]{2}{*}{$\begin{array}{c}\text { Det } \\
\text { Veloc. } \\
\text { Ratio } \\
\mathrm{U}_{\mathrm{sf}} \mathrm{D}\end{array}$} & \multicolumn{2}{|c|}{ Smallest } & Max. & \multirow[b]{2}{*}{$\begin{array}{c}\left\langle\mathrm{x}_{\mathrm{e}}\right\rangle \\
\text { at } \infty \\
(\mathrm{mm})\end{array}$} & \multirow[b]{2}{*}{$\begin{array}{l}\text { Length } \\
(\mathrm{mm})\end{array}$} & \multirow[b]{2}{*}{ Ref. } & \multirow[b]{2}{*}{ Remarks } \\
\hline & & & $\begin{array}{c}\text { Radius } \\
\mathbf{R}_{\mathrm{f}} \\
(\mathrm{mm})\end{array}$ & $\begin{array}{r}\text { Deto } \\
\text { Vel } \\
\text { (m }\end{array}$ & $\begin{array}{l}\text { ation } \\
\text { ity } \\
\mu \mathrm{s})\end{array}$ & & & & \\
\hline$\overline{\mathrm{XTX}}-8003$ & 1.53 & 0.97 & 0.095 & 7.09 & 7.30 & 0.04 & 200 & 1 & PETN \\
\hline PBX-9501 & 1.83 & 0.94 & 0.79 & 8.26 & 8.80 & 0.1 & 47 & 1 & HMX 95 \\
\hline NM/ PMMA/Al & 1.18 & 0.996 & 0.55 & 6.19 & 6.22 & 0.15 & 290 & 2 & $5 \% \mathrm{Al}, \mathrm{con}$ \\
\hline NM/ PMMA/Al & 1.22 & 0.995 & 0.55 & 6.13 & 6.16 & 0.15 & 290 & 2 & $10 \% \mathrm{Al}, \mathrm{con}$ \\
\hline NM/ PMMA/Al & 1.25 & 0.995 & 0.55 & 6.08 & 6.10 & 0.15 & 290 & 2 & $15 \% \mathrm{Al}$, con \\
\hline Comp A & 1.69 & 0.985 & 2.12 & 8.14 & 8.27 & 0.2 & - & 1 & $\mathrm{RDX}$ \\
\hline Comp B, Type 4 & 1.70 & 0.94 & 2.56 & 7.46 & 7.90 & 0.2 & & 3 & RDX/ TNT \\
\hline Liquid TNT, unc. & & 0.993 & 3.25 & 6.52 & 6.56 & 0.2 & 500 & 4 & $100^{\circ} \mathrm{C}$ \\
\hline Comp B, Type 1 & 1.70 & 0.85 & 2.13 & 6.70 & 7.87 & 0.3 & 50 & 3 & RDX/TNT \\
\hline PBX-9404 & 1.84 & 0.827 & 0.6 & 7.28 & 8.81 & 0.5 & 25 & 1 & HMX 94 \\
\hline Liquid TNT & & 0.993 & 0.95 & 6.52 & 6.56 & 0.5 & $8 R_{0}$ & 4 & $\operatorname{con}, 100^{\circ} \mathrm{C}$ \\
\hline NM confined & 1.15 & 0.981 & 0.55 & 6.19 & 6.32 & 0.5 & 290 & 2 & with PMMA \\
\hline NM confined & 1.03 & 0.986 & 1.5 & 6.13 & 6.21 & 0.75 & 775 & 1 & NM \\
\hline PBX-9502, $75^{\circ} \mathrm{C}$ & 1.89 & 0.96 & 3.0 & 7.38 & 7.66 & 0.6 & $50^{\circ}$ & 5 & TATB \\
\hline Octol & 1.82 & 0.96 & 3.2 & 8.16 & 8.47 & 0.8 & 510 & 1 & HMX/ TNT \\
\hline $\mathrm{X}-0219$ & 1.91 & 0.975 & 8.0 & 7.38 & 7.57 & 0.8 & 300 & 1 & TATB \\
\hline Comp B, Type 2 & 1.70 & 0.93 & 3.56 & 7.27 & 7.82 & 0.9 & 60 & 3 & $\mathrm{RDX} / \mathrm{TNT}$ \\
\hline Comp B, Type 3 & 1.70 & 0.92 & 2.35 & 7.36 & 8.00 & 0.9 & & 3 & \\
\hline TNT, pressed & 1.62 & 0.93 & 1.576 & 6.617 & 7.08 & 1.2 & & 6 & \\
\hline $\mathrm{X}-0344$ & 1.89 & 0.97 & 2.9 & 7.81 & 8.07 & 1.2 & & 7 & TATB/HMX \\
\hline TNT, pressed & 1.55 & 0.92 & 1.6 & 6.33 & 6.91 & 1.4 & & 6 & \\
\hline $\mathrm{X}-0343$ & 1.90 & 0.96 & 2.9 & 7.60 & 7.93 & 1.4 & & 7 & TATB/HMX \\
\hline Cyclotol & 1.74 & 0.92 & 3.2 & 7.66 & 8.29 & 1.6 & - & 1 & RDX / TNT \\
\hline$x-0290$ & & 0.89 & 5 & 6.9 & 7.71 & 1.7 & - & 1 & TATB \\
\hline $\mathrm{X}-0342$ & 1.90 & 0.95 & 3.5 & 7.50 & 7.88 & 1.7 & & 7 & TATB/HMX \\
\hline $\mathrm{X}-0341$ & 1.90 & 0.95 & 4.0 & 7.4 & 7.83 & 2.0 & & 7 & TATB/HMX \\
\hline PBX-9502 & 1.89 & 0.97 & 5.0 & 7.41 & 7.65 & 2.0 & 300 & 5 & TATB \\
\hline PBX-9502, $-55^{\circ} \mathrm{C}$ & 1.89 & 0.96 & 5.2 & 7.40 & 7.73 & 2.0 & 50 & 5 & TATB \\
\hline TNT, pressed & 1.46 & 0.87 & 2.4 & 5.76 & 6.59 & 2 & & 6 & \\
\hline $\mathrm{X}-0309$ & 1.70 & 0.97 & 15.9 & 6.56 & 6.77 & 2.2 & 380 & 1 & TNT \\
\hline TNT, loose & 1.00 & 0.70 & 4.5 & 3.42 & 4.86 & 3 & & 6 & \\
\hline $\mathrm{AP} 90, \mathrm{Al} 10$ & 0.90 & 0.85 & 12.7 & 3.33 & 3.94 & 5 & & 8 & \\
\hline $\mathrm{AP} 90, \mathrm{Al} 10$ & 1.10 & 0.86 & 12.7 & 3.79 & 4.40 & 5 & & 8 & \\
\hline QM-100 & 1.26 & 0.52 & 19 & 3.3 & 6.25 & 5 & - & 9 & $\mathrm{ANFO} / \mathrm{H} 2 \mathrm{O}$ \\
\hline TNT, loose & 0.80 & 0.43 & 4.0 & 1.86 & 4.31 & 5 & & 6 & \\
\hline $\mathrm{AP} 90, \mathrm{Al} 10$ & 0.62 & 0.89 & 12.7 & 2.72 & 3.05 & 6 & & 8 & \\
\hline Comp B/AN, 71/29 & 1.59 & 0.87 & 6.8 & 6.72 & 7.70 & 6 & & 10 & \\
\hline
\end{tabular}


Table 8-2, part 2

\begin{tabular}{|c|c|c|c|c|c|c|c|c|c|}
\hline \multirow[b]{2}{*}{ Explosive } & \multirow[b]{2}{*}{$\begin{array}{l}\text { Den- } \\
\text { sity } \\
(\mathrm{g} / \mathrm{cc})\end{array}$} & \multirow{2}{*}{$\begin{array}{c}\text { Det } \\
\text { Veloc. } \\
\text { Ratio } \\
\mathrm{U}_{\mathrm{sf}} \mathrm{D}\end{array}$} & \multicolumn{2}{|c|}{ Smallest } & Max. & \multirow[b]{2}{*}{$\begin{array}{c}\langle x e> \\
\text { at } \infty \\
(\mathrm{mm})\end{array}$} & \multirow[b]{2}{*}{$\begin{array}{c}\text { Length } \\
(\mathrm{mm})\end{array}$} & \multirow[b]{2}{*}{ Ref. } & \multirow[b]{2}{*}{ Remarks } \\
\hline & & & $\begin{array}{c}\text { Radius } \\
\mathrm{R}_{\mathrm{f}} \\
(\mathrm{mm})\end{array}$ & & $\begin{array}{l}\text { ation } \\
\text { city } \\
\mu s)\end{array}$ & & & & \\
\hline ANFO & 0.95 & 0.69 & 12.15 & 3.24 & 4.72 & 7 & & 11 & AN/ FO \\
\hline $\mathrm{AP} 90, \mathrm{Al} 10$ & 1.36 & 0.77 & 12.7 & 4.11 & 5.31 & 7 & & 8 & \\
\hline AN/TNT, $50-50$ & 1.00 & 0.40 & 6 & 1.8 & 4.40 & 8 & & 10 & \\
\hline $\mathrm{AP}$, loose & 1.00 & 0.62 & 17.1 & 2.3 & 3.66 & 9 & & 12 & \\
\hline PBXN-111 & 1.79 & 0.84 & 19.4 & 5.04 & 6.03 & 9 & 153 & $\begin{array}{l}13- \\
15\end{array}$ & $\mathrm{AP} / \mathrm{Al} / \mathrm{RDX}$ \\
\hline TNT/AN, 50/50 & 1.00 & 0.41 & 6.4 & 1.82 & 4.40 & 9 & & 10 & \\
\hline AN/TNT, $80 / 20$ & 1.61 & 0.85 & 12.7 & 6.53 & 7.67 & 10 & 400 & 1 & \\
\hline AP & 1.24 & 0.62 & 12.7 & 2.65 & 4.30 & 10 & & 12 & $\mathrm{AP}$ \\
\hline ANFO & 0.80 & 0.69 & 25.5 & 3.25 & 4.74 & 11 & 300 & $\begin{array}{r}16 \\
17\end{array}$ & $\mathrm{AN} / \mathrm{FO}$ \\
\hline ANFO & 1.25 & 0.62 & 15.95 & 4.0 & 6.45 & 14 & $610-1520$ & 18 & $\mathrm{ANFO} / \mathrm{H} 2 \mathrm{O}$ \\
\hline TNT/AN, 50/50 & 1.53 & 0.69 & 18.7 & 4.88 & 7.05 & 14 & & 10 & \\
\hline $\mathrm{DNT}, 175 \mu \mathrm{m}$ & 0.95 & 0.57 & 25.1 & 2.39 & 4.21 & 15 & & 19 & \\
\hline HANFO & 1.06 & 0.36 & 30.3 & 1.9 & 5.35 & 25 & 1000 & 20 & $\mathrm{ANFO} / \mathrm{H} 2 \mathrm{O}$ \\
\hline RX-HD & 1.32 & 0.61 & 62 & 4.2 & 6.90 & 25 & & 21, & $\mathrm{ANFO} / \mathrm{H} 2 \mathrm{O}$ \\
\hline $\mathrm{AN}, 200 \mu \mathrm{m}$ & 1.04 & 0.42 & 85 & 1.4 & 3.30 & 50 & & 23 & \\
\hline
\end{tabular}


Table 8-3. Detonation curvature constants for various explosives. The ordering by increasing values of the relative measured lag at the edge of the cylinder, $\mathrm{L}_{0} / R_{0}$. " $C$ " is confined; " $U$ " is unconfined.

\begin{tabular}{|c|c|c|c|c|c|c|c|c|c|c|}
\hline \multirow[b]{2}{*}{ Explosive } & \multirow{2}{*}{$\begin{array}{l}\text { Den- } \\
\text { sity } \\
(\mathrm{g} / \mathrm{cc})\end{array}$} & & \multirow{2}{*}{$\begin{array}{c}\text { Expl. } \\
\text { Radius } \\
R_{o} \\
(\mathrm{~mm})\end{array}$} & \multirow{2}{*}{$\begin{array}{l}\text { Meas. } \\
\text { Lag, } \\
\mathrm{L}_{\mathrm{o}} \\
(\mathrm{mm})\end{array}$} & \multirow{2}{*}{$\begin{array}{c}\text { Meas. } \\
\text { Rel. } \\
\text { Gap } \\
\mathrm{L}_{\mathrm{d}} / \mathrm{R}_{\mathrm{o}}\end{array}$} & \multicolumn{2}{|c|}{$\begin{array}{l}\text { Edge Angle, } \\
\Theta \text { (degree) }\end{array}$} & \multicolumn{2}{|c|}{$\begin{array}{l}\text { Quadratic } \\
\text { Constants }\end{array}$} & \multirow{2}{*}{$\begin{array}{c}\mathrm{F}_{\mathrm{u}} \\
\text { (edge) }\end{array}$} \\
\hline & & & & & & Meas & Quad & $\mathrm{A}$ & $\mathrm{AR}_{\mathrm{o}}$ & \\
\hline LX-10 & 1.86 & $\mathrm{C}$ & 23.4 & 0.044 & 0.0019 & 90 & 90 & 0.00003 & 0.0007 & \\
\hline $\mathrm{AP}$ & 1.00 & $\mathrm{U}$ & 25.0 & 0.047 & 0.0019 & 90 & 90 & 0.00007 & 0.0017 & \\
\hline $\mathrm{AP}$ & 1.40 & $U$ & 25.4 & 0.059 & 0.0023 & 90 & 90 & 0.00011 & 0.0028 & \\
\hline LXX-04 & 1.87 & $\mathrm{C}$ & 23.1 & 0.28 & 0.012 & 86 & 89 & 0.00034 & 0.0079 & \\
\hline $\mathrm{T} 2$ & 1.86 & $\mathrm{U}$ & 50 & 2.92 & 0.058 & 81 & 84 & 0.0011 & 0.055 & \\
\hline $\mathrm{RX}-52-\mathrm{AD}$ & 1.77 & $\mathrm{C}$ & 25.4 & 1.69 & 0.066 & 77 & 83 & 0.0024 & 0.061 & \\
\hline $\mathrm{RX}-52-\mathrm{AE}$ & 1.78 & $\mathrm{C}$ & 25.2 & 1.73 & 0.069 & 79 & 83 & 0.0024 & 0.060 & \\
\hline $\mathrm{LX}-17$ & 1.91 & $\mathrm{C}$ & 23.4 & 1.72 & 0.073 & 79 & 82 & 0.0029 & 0.068 & \\
\hline Comp B & 1.67 & $\mathrm{U}$ & 25.43 & 1.88 & 0.074 & 80 & 82 & 0.0028 & 0.071 & \\
\hline IRX-3A & & $\mathrm{U}$ & 25 & 1.9 & 0.076 & 70 & 83 & 0.0024 & 0.060 & \\
\hline $\mathrm{T} 2$ & 1.86 & $\mathrm{U}$ & 25 & 1.92 & 0.077 & 79 & 82 & 0.0027 & 0.068 & \\
\hline Comp B & 1.67 & $\mathrm{C}$ & 25.43 & 2.0 & 0.077 & 79 & 82 & 0.00293 & 0.075 & \\
\hline PBX-9502 & 1.89 & $\mathrm{U}$ & 24.9 & 2.14 & 0.086 & 66 & 82 & 0.0029 & 0.072 & \\
\hline IRX-1 & & $\mathrm{U}$ & 25 & 2.4 & 0.095 & 73 & 80 & 0.0035 & 0.088 & \\
\hline PBXN-110 & 1.68 & $\mathrm{U}$ & 24.95 & 2.53 & 0.101 & 68 & 81 & 0.0031 & 0.077 & \\
\hline PBX-9502 & 1.89 & $\mathrm{U}$ & 9.0 & 0.95 & 0.106 & 69 & 80 & 0.0101 & 0.091 & \\
\hline Octol & 1.81 & $\mathrm{C}$ & 12.7 & 1.37 & 0.108 & NG & 84 & 0.0042 & 0.053 & \\
\hline PBXN-111 & 1.79 & $\mathrm{C}$ & 23.5 & 2.72 & 0.116 & 74 & 78 & 0.0045 & 0.106 & 0.35 \\
\hline PBX-9502 & 1.89 & $\mathrm{U}$ & 6.0 & 0.71 & 0.118 & 72 & 78 & 0.0172 & 0.103 & \\
\hline PBXN-111 & 1.79 & $\mathrm{C}$ & 33.96 & 4.1 & 0.121 & 74 & 77 & 0.0033 & 0.112 & 0.31 \\
\hline PBXN-111 & 1.79 & $\mathrm{C}$ & 24.23 & 3.0 & 0.124 & 75 & 76 & 0.0052 & 0.126 & 0.33 \\
\hline IRX-4 & 1.50 & $\mathrm{U}$ & 24.4 & 3.76 & 0.154 & 62 & 76 & 0.0052 & 0.127 & 0.18 \\
\hline PBXN-111 & 1.79 & U & 34.13 & 5.75 & 0.168 & 62 & 73 & 0.0046 & 0.157 & 0.32 \\
\hline ANFO & 1.248 & $\mathrm{U}$ & 51.15 & 8.67 & 0.170 & 64 & 73 & 0.00305 & 0.156 & 0.56 \\
\hline PBXW-123* & 1.92 & $\mathrm{C}$ & 38.5 & 6.55 & 0.170 & 65 & 71 & 0.0044 & 0.169 & 0.32 \\
\hline ANFO & 1.248 & $\mathrm{U}$ & 38.95 & 7.15 & 0.184 & 63 & 71 & 0.00439 & 0.171 & 0.74 \\
\hline ANFO & 1.248 & $\mathrm{U}$ & 20.45 & 4.05 & 0.198 & 60 & 70 & 0.00910 & 0.186 & 0.34 \\
\hline PBXN-111 & 1.79 & $U$ & 24.01 & 4.8 & 0.201 & 61 & 68 & 0.0085 & 0.204 & 0.34 \\
\hline PBXN-111 & 1.79 & $\mathrm{U}$ & 24.06 & 4.86 & 0.202 & 60 & 69 & 0.0080 & 0.192 & 0.35 \\
\hline PBXN-111 & 1.79 & $\mathrm{U}$ & 20.52 & 4.29 & 0.209 & 62 & 68 & 0.0098 & 0.201 & 0.38 \\
\hline Pentolite & 1.56 & U & 25.4 & 5.70 & 0.224 & 68 & 66 & 0.0089 & 0.226 & \\
\hline HANFO & 1.07 & $\mathrm{U}$ & 126.85 & 29.1 & 0.229 & 52 & 67 & 0.0017 & 0.216 & 0.37 \\
\hline PBXN-111 & 1.79 & $\mathrm{U}$ & 20.45 & 4.81 & 0.235 & 59 & 65 & 0.0112 & 0.229 & 0.36 \\
\hline HANFO & 1.06 & $\mathrm{U}$ & 76.35 & 18.6 & 0.244 & 55 & 68 & 0.0027 & 0.206 & 0.64 \\
\hline HANFO & 1.06 & $\mathrm{U}$ & 60.05 & 15.4 & 0.256 & $N G$ & 66 & 0.0038 & 0.228 & 0.71 \\
\hline HANFO & 1.07 & $\mathrm{U}$ & 40.2 & 12.2 & 0.304 & & & & & \\
\hline
\end{tabular}

*probably a decaying detonation 
Table 8-3, part 2

\begin{tabular}{|c|c|c|c|c|c|}
\hline Reaction Zone & Meas & $\mathrm{U}_{\mathrm{t}}(\max )$ & D & & \\
\hline$\left\langle\mathrm{x}_{\mathrm{e}}\right\rangle(\mathrm{mm})$ & $\mathrm{U}_{\mathrm{s}}$ & $(\mathrm{mm} / \mu \mathrm{s})$ & at $\infty$ & Elliptical & Elliptical \\
\hline Inverse $\quad$ f. Det & $(\mathrm{mm} /$ & om from & $(\mathrm{mm} /$ & Constants & Focus \\
\hline
\end{tabular}

\begin{tabular}{|c|c|c|c|c|c|c|c|c|c|c|c|}
\hline Explosive & Radius & Lag & $\mu s)$ & $\mathrm{D}^{2}$ & angle & $\mu s)$ & $\mathrm{a} / \mathrm{R}_{0}$ & $\mathrm{~b} / \mathrm{R}_{\mathrm{o}}$ & $(\mathrm{mm})$ & (deg) & Ref. \\
\hline LX-10 & 0.1 & 0.008 & 8.82 & & 0.01 & 8.82 & 7.91 & 0.085 & 185 & 90 & 24 \\
\hline $\mathrm{AP}$ & 7 & 0.003 & 3.66 & & & 3.66 & 12.0 & 0.489 & 300 & 90 & 25 \\
\hline $\mathrm{AP}$ & 10 & 0.004 & 4.82 & & & 4.80 & 9.1 & 0.469 & 230 & 89 & 25 \\
\hline LX-04 & 0.1 & 0.31 & 8.46 & & 0.13 & 8.46 & 6.45 & 0.723 & 148 & 88 & 24 \\
\hline $\mathrm{T} 2$ & 2 & 2.7 & 7.63 & & 0.84 & 7.63 & 1.50 & 0.220 & 74 & 79 & 26 \\
\hline $\mathrm{RX}-52$ & & 3.9 & 7.55 & & 0.93 & 7.55 & 2.40 & 0.673 & 58 & 77 & 24 \\
\hline $\mathrm{RX}-52$ & & 4.0 & 7.57 & & 0.92 & 7.57 & 2.38 & 0.679 & 58 & 77 & 24 \\
\hline LX-17 & 2 & 4.1 & 7.63 & & 1.03 & 7.63 & 2.36 & 0.739 & 52 & 75 & 24 \\
\hline Cmp B & $0.3-0.9$ & 2.4 & 7.86 & 0.68 & 1.12 & 7.89 & 2.02 & 0.554 & 49 & 75 & 14,27 \\
\hline IRX-3A & 0.5 & 2.4 & 7.79 & 1.14 & 0.93 & 7.87 & 1.88 & 0.404 & 46 & 77 & 28 \\
\hline $\mathrm{T} 2$ & 2 & 2.5 & 7.62 & & 1.03 & 7.62 & 1.28 & 0.193 & 32 & 77 & 26 \\
\hline Cmp B & $0.3-0.9$ & 4.5 & 7.86 & 0.35 & 1.17 & 7.87 & 2.04 & 0.590 & 50 & 74 & 14 \\
\hline 9502 & 2.0 & 2.8 & 7.67 & 0.99 & 1.11 & 7.73 & 1.23 & 0.191 & 30 & 76 & 29 \\
\hline IRX-1 & & 3.2 & 7.49 & 1.65 & 1.31 & 7.67 & 1.60 & 0.404 & 39 & 72 & 28 \\
\hline$N-110$ & & 3.4 & 8.39 & & 1.30 & 8.39 & 1.64 & 0.389 & 40 & 74 & 30 \\
\hline 9502 & 2.0 & 1.9 & 7.55 & 1.63 & 1.37 & 7.73 & 2.21 & 0.892 & 18 & 70 & 29 \\
\hline Octol & 0.8 & 4.4 & 8.42 & 0.87 & & 8.46 & 2.29 & 0.642 & 28 & 76 & 24 \\
\hline N-111 & 15 & 12 & 5.60 & & 1.18 & 7.08 & 2.27 & 1.04 & 47 & 68 & 14,31 \\
\hline 9502 & 1.8 & 1.7 & 7.50 & 1.86 & 1.54 & 7.73 & 2.53 & 1.34 & 13 & 67 & 29 \\
\hline $\mathrm{N}-111$ & 15 & 14 & 5.76 & & 1.29 & 7.08 & 1.26 & 0.306 & 42 & 69 & 14,31 \\
\hline N-111 & 15 & 12 & 5.61 & & 1.41 & 7.08 & 1.42 & 0.442 & 33 & 66 & 14,31 \\
\hline IRX-4 & & 8 & 5.62 & & 1.43 & 6.42 & 1.35 & 0.410 & 31 & 66 & 30 \\
\hline $\mathrm{N}-111$ & 15 & 12 & 5.57 & & 1.75 & 7.08 & 1.43 & 0.568 & 45 & 61 & 14,32 \\
\hline ANFO & 16 & 20 & 4.30 & & 1.34 & 6.79 & 1.17 & 0.353 & 57 & 63 & 18 \\
\hline W-123 & & 15 & 5.56 & & 1.88 & 7.12 & 1.03 & 0.278 & 38 & 62 & 33 \\
\hline ANFO & 17 & 21 & 3.28 & & 1.12 & 6.79 & 1.16 & 0.376 & 43 & 61 & 18 \\
\hline ANFO & 19 & 10 & 5.15 & & 1.92 & 6.79 & 1.47 & 0.724 & 26 & 56 & 18 \\
\hline N-111 & 15 & 11 & 5.31 & & 2.17 & 7.08 & 1.70 & 1.08 & 31 & 53 & 14,32 \\
\hline $\mathrm{N}-111$ & 15 & 11 & 5.31 & & 2.04 & 7.08 & 1.51 & 0.781 & 31 & 56 & 14,32 \\
\hline$N-111$ & 15 & 10 & 5.16 & & 2.07 & 7.08 & 1.22 & 0.497 & 23 & 56 & 14,32 \\
\hline Pentolite & & 7 & 7.19 & & 3.25 & 7.19 & 1.19 & 0.539 & 27 & 53 & 30 \\
\hline HANFO & 34 & 35 & 4.30 & & 1.85 & 5.89 & 1.18 & 0.512 & 135 & 54 & 20 \\
\hline $\mathrm{N}-111$ & 15 & 10 & 5.15 & & 2.36 & 7.08 & 1.17 & 0.504 & 22 & 54 & 14,32 \\
\hline HANFO & 34 & 35 & 3.28 & & 1.35 & 5.89 & 1.31 & 0.616 & 88 & 54 & 20 \\
\hline HANFO & 31 & 31 & 2.90 & & 1.32 & 5.89 & 1.67 & 1.199 & 69 & 49 & 20 \\
\hline HANFO & & & 2.39 & & & 5.89 & & & & & 20 \\
\hline
\end{tabular}





\section{Chapter 9. More Reaction Zones}

\section{Time Constant on the Principal Rayleigh Line}

The Principal Rayleigh Line defines full detonation (connecting to the "C-J" point). The time for the detonation to turn on near steady state has been studied by means of direct gauge measurements of the particle velocity. The first two runs by Hayes and Tarver used a point detonator driving a $25 \mathrm{~mm}$ TNT booster which drove $62 \mathrm{~mm}$ of either LX-14 or LX-17. 1,2 The $25 \mu \mathrm{m}$ copper gauges were placed $12 \mathrm{~mm}$ before the end, so the final explosive run distance was $50 \mathrm{~mm}$. The sample diameter was $51 \mathrm{~mm}$. In the other set of experiments by Urtiew, two-inch diameter cylinders of explosive were allowed to run 20 to $25 \mathrm{~mm}$ before encountering the gauge with 8 to $15 \mathrm{~mm}$ of remaining explosive. The LX-17 was boosted with $5 \mathrm{~mm}$ of $\mathrm{LX}-10$ or RX-26-AF. 3

The best set of data, for RX-26-AH, is shown in Fig. 9-1. The rise time is assumed to have the form

$$
\mathrm{u} \approx \mathrm{u}_{\max }\left[1-\exp \left(-\mathrm{t} / \tau_{\mathrm{ss}}\right)\right]
$$

The maximum value measured is $\mathbf{u}_{\max }$. Thereafter, the particle velocity declines as the products relax down the adiabat. The summarized results are in Table 9-1, where $\tau_{\mathrm{SS}}$ near steady state is about $0.02 \mu \mathrm{s}$. The fact that all explosives show the same result, despite their obvious differences, suggest that we are looking at the turn-on of the gauges. ${ }^{3}$ We may suggest that the time constant to steady state, $t_{\mathrm{SS}}$, of these explosives on the Principal Rayleigh Line is

$$
\tau_{\mathrm{SS}}<20 \mathrm{~ns}
$$

\section{Walker-Wasley and Reaction Zones}

We now want to extend our analysis below steady state detonation, but we first need to develop some new ideas. The well-known Walker-Wasley rule sought to find an input energy that created detonation. 4 We shall try a different approach with the same equation. Consider a burn with the exponential form 


$$
F=1-\exp \left(-4 x / x_{r}\right)
$$

It will take 4 to 5 reaction zone lengths, $x_{r}$, for $F$ to reach about 1 . Because the burn goes exponentially, we try the idea that detonation will occur if we supply the compression energy for the distance $x_{r} / 4$. If $P$ is the initation pressure, we now substitute the total energy of compression, $2 \mathrm{E}_{\mathrm{c}}$ (the sum of the internal and kinetic energies):

$$
\mathrm{E}(\mathrm{in}) \approx \mathrm{E}_{\mathrm{c}} \mathrm{x}_{\mathrm{r}} / 2=\mathrm{P}(1-\mathrm{v}) \mathrm{x}_{\mathrm{r}} / 2 \text {. }
$$

$\mathrm{E}_{\mathrm{c}}$ is an energy per cc where we assume a $1 \mathrm{~cm}^{2}$ area. The time to move this distance is

$$
t=x_{r} / 4 U_{S}
$$

We also need the Rayleigh Line

$$
1-\mathrm{v}=\mathrm{P} / \mathrm{\rho}_{\mathrm{O}} \mathrm{U}_{\mathrm{S}}{ }^{2}
$$

We substitute these into Eq. 4 to get the Walker-Wasley relation

$$
E(i n)=\frac{2 P^{2} \tau}{\rho_{o} U_{s}}
$$

Walker and Wasley interpreted E(in) as being per a critical energy unit area impacted, rather than an energy per unit volume. to be put in. Given the mix of variables, there is no reason to expect that $E$ (in) should be a constant over a range of pressures.

\section{Run-to-Detonation Distance}

The important part of the Walker-Wasley relation is the idea that we need to keep a "thick" pulse on for a time $t$ so it can compress a distance $x_{r} / 4$. The place to go to develop this is the run-todetonation distance (Pop plot) obtained from wedge tests. Here, the initiation pressure of the thick pulse is related to a run distance, $x$, and a run time, $t$, by

$$
\log \mathrm{P}=\mathrm{J}-\mathrm{K} \log \mathrm{x}
$$

where $\mathrm{J}$ and $\mathrm{K}$ are positive constants. A new and better fit has been found by Cooper: ${ }^{5}$ 


$$
\mathrm{p}^{2} \mathrm{x}=\mathrm{a}
$$

where $a$ is a constant for each explosive over the range of initiation pressures.

In Table 9-2, 6-9 we include the constants for the two run-to-detonation equations and extrapolate to the approximate detonation pressures. We find that the reaction zone at steady state is well approximated by the relation

$$
\mathbf{x}_{\mathbf{r}}{ }^{0} \approx \mathbf{x} / 4
$$

The extrapolation process works better with the Cooper equation for explosives with small reaction zones.

If thin-pulse initiation is present, then the distance-to-detonation rises rapidly while the initation pressure declines slightly. So the measured minimum distance at a given pressure is going to be close to the thick-pulse result. ${ }^{8}$ Put another way, if we do not hold the pulse on long enough to cover the true $\mathrm{x}_{\mathrm{r}} / 4$, detonation may still occur, but $\mathrm{x}_{\mathrm{r}} / 4$ gets stretched out much longer.

\section{Reaction Zones in Ignition \& Growth (with Craig Tarver)}

Ignition \& Growth (I\&G) offers the only time-dependent view in the codes. We consider the messy composite RX-25-BF/BP, a mixture of HMX, AP and $\mathrm{ZrH}_{2}$ using DYNA2D with square zones $0.25 \mathrm{~mm}$ on a side. 10 Here, we expect both a big reaction zone and a large curvature. Fig. 9-2 shows the I\&G results, using the usual 3-term version on unconfined cylinders, for the $3.18 \mathrm{~mm}$ radius, which is close to the failure radius. The shock wave is thin but the reaction zone, taken here as the $0.1<\mathrm{F}<0.9$ distance, is much longer. The pressure drops rapidly behind the shock wave and only about 0.25 of the explosive has reacted at the maximum pressure. This is a perfect example of an explosive with a spike pressure but no CJ point. Because JWL's are used, there is a tendency to nevertheless list the reacted C-J pressure of $0.28 \mathrm{Mb}$, which is nothing more than a fictional marker in this problem. The reaction zone may be measured along the axis to which it is parallel. The reaction zone length out to the edge lies at a steep angle to the direction of detonation.

This problem was rerun in DYNA2D for unconfined cylinder radii of $3.18,6.35$ and $25.4 \mathrm{~mm}$ with 64 zones across the radius. The output was taken $35 \mathrm{~mm}$ deep into the explosive after assuring that it was at steady state. The results are seen below. 


\begin{tabular}{|c|c|c|c|c|}
\hline \multirow{2}{*}{$\begin{array}{c}\text { Outer } \\
\text { Radius } \\
\mathbf{R}_{\mathbf{o}}(\mathrm{mm})\end{array}$} & \multirow{2}{*}{$\begin{array}{c}\mathrm{Lag} \\
\mathrm{L}_{0} \\
(\mathrm{~mm})\end{array}$} & \multirow{2}{*}{$\begin{array}{l}\text { Ratio } \\
\mathrm{L}_{0} / \mathrm{R}_{\mathrm{o}}\end{array}$} & \multicolumn{2}{|c|}{$\begin{array}{l}\text { Reaction Zone, } \mathrm{x}_{\mathbf{r}} \\
(\mathrm{mm}), 0<\mathrm{F}<0.9\end{array}$} \\
\hline & & & center & edge \\
\hline 25.4 & 2.15 & 0.085 & 1.5 & 2.0 \\
\hline 6.35 & 1.45 & 0.23 & 2.2 & 3.4 \\
\hline 3.175 & 0.9 & 0.27 & 3.5 & 3.6 \\
\hline
\end{tabular}

We see that the absolute value of the edge lag increases with the radius, but the relative lag, $L_{0} / R_{0}$, increases with decreasing radius. As the radius decreases, the reaction zone stretches out with most of the burn in the early part. The reaction zone ratio of the edge to the center is larger for the large radii. In this case, the center reaction zone is close to complete confinement whereas the edge is at a much lower pressure. For a small radius, the entire sample is detonating at a lower pressure. The reaction zone is so stretched out that a little more doesn't matter.

The detonation pressure varies across the shock front. Fig. 9-3 shows the calculated detonation front at the points of maximum pressure along with the burn fraction. Both are close to being constant across much of the front, but they then drop precipitously near the edge. This qualitatively agrees with the Inverse Radius model (Chapter 8 ) and the edge layer of thickness $R_{e}$ is fairly easy to see. The pressure crudely goes as

$$
P_{\max }-P \approx R^{4}
$$

where the relation fails for the last few zones near the edge. The detonation lag, however, builds up gradually across the radius and fits an $\mathrm{R}^{2}$ function quite well.

From Chapter 8, we write for the detonation front

$$
E_{0}-<E_{o}>=\left(\frac{E_{o}}{D^{2}}\right)\left(D^{2}-U_{s}^{2}\right)=\left(\frac{E_{0}}{D^{2}}\right) U_{t}(\max )^{2} \approx \frac{1}{2} \Delta P_{\max }(1-v)
$$

where $v$ is considered constant. The energy difference is going sideways out of the cylinder and the result is a lower pressure at the edge. We generalize this across the detonation front to

$$
\frac{1}{2} P_{\max }(1-v) \approx \frac{1}{2} P(1-v)+\left(\frac{E_{o}}{D^{2}}\right) U_{t}^{2}
$$


Because $U_{t} \approx R^{2}$, we derive the fourth power relationship of Eq. 12 .

\section{Simplest I\&G Rate Term that causes Curvature (with Craig Tarver)}

We are interested in reproducing the detonation front curvature in a steady state cylinder using the simplest possible rate equation. A possible form is to use only one growth term with no initiation. Then, we have

$$
\frac{\partial \mathrm{F}}{\partial \mathrm{t}}=\mathrm{AP}
$$

Below, we compare the results for unconfined RX-25-BP for the original 3-term I\&G with the model running with various values of $A$, with the same unreacted and reacted JWL's in place. The value of $\mathrm{A}=15(\mathrm{Mb} \cdot \mu \mathrm{s})^{-1}$ is the closest to reproducing the original run as far as the lag and the reaction zone are concerned. The reaction zone with Eq. 15 does not change across the front, whereas we has a factor of 2 before, but we could not measure this difference with present technology anyway. We see that both the maximum lag and the reaction zone decrease with increasing $A$.

\begin{tabular}{|c|c|c|c|c|c|c|c|c|}
\hline \multirow{2}{*}{$\begin{array}{l}\text { Model } \\
\text { Type }\end{array}$} & \multirow[b]{2}{*}{ Coeff } & \multirow{2}{*}{$\begin{array}{c}\text { Lag } \\
\mathrm{L}_{0} \\
(\mathrm{~mm})\end{array}$} & \multirow{2}{*}{$\begin{array}{l}\text { Ratio } \\
\mathrm{L}_{0} / \mathrm{R}_{0}\end{array}$} & \multicolumn{2}{|c|}{$\begin{array}{l}\text { Reaction Zone, } \mathrm{x}_{\mathbf{r}} \\
(\mathrm{mm}), 0<\mathrm{F}<0.9\end{array}$} & \multirow{2}{*}{$\begin{array}{l}\text { Edge-to } \\
\text { Center } \\
\text { Ratio }\end{array}$} & \multirow{2}{*}{$\begin{array}{c}P_{k} \\
(\mathrm{Mb})\end{array}$} & \multirow{2}{*}{$\begin{array}{c}\text { Meas } \\
\mathrm{F}_{1} \text { at } \\
\mathrm{P}_{\mathrm{k}} \\
\end{array}$} \\
\hline & & & & center & edge & & & \\
\hline I\&G & I\&G & 2.2 & 0.08 & 1.5 & 2.0 & 1.3 & 0.35 & 0.3 \\
\hline $\mathrm{AP}$ & 2 & 5.5 & 0.22 & 9.4 & 11 & 1.2 & 0.28 & 0.18 \\
\hline $\mathrm{AP}$ & 5 & 4.2 & 0.17 & 3.2 & 4.8 & 1.5 & 0.39 & 0.3 \\
\hline $\mathrm{AP}$ & 10 & 3.0 & 0.12 & 2.1 & 2.8 & 1.3 & 0.40 & 0.3 \\
\hline $\mathrm{AP}$ & 15 & 2.3 & 0.09 & 1.7 & 2.2 & 1.3 & 0.40 & 0.3 \\
\hline $\mathbf{A P}$ & 20 & 1.8 & 0.07 & 1.5 & 2.0 & 1.3 & 0.42 & 0.3 \\
\hline $\mathrm{BP}^{2}$ & 55 & 2.7 & 0.11 & 1.4 & $\overline{2.4}$ & 1.7 & & \\
\hline
\end{tabular}

Suppose the pressure initially rises exponentially as

$$
P=0.368 P_{k} \exp \left(t / \tau_{1}\right)
$$

where $P_{k}$ is the maximum spike pressure, $\tau_{1}$ is the turn-on time constant. and the time will run for one time constant, which is the reason for the 0.368 . The amount burned after the time $\tau_{1}$ is

$$
F_{1}=A P_{k} \tau_{1} .
$$


For $\mathrm{RX}-25-\mathrm{BD}$, we have $\mathrm{A} \approx 15(\mathrm{Mb} \cdot \mu \mathrm{s})^{-1}$ and $\mathrm{F}_{1} \approx 0.3$; then we get

$$
\tau_{1} \approx 50 \mathrm{~ns}
$$

This agrees with the steady state time constant measured in the first section above. The pressure rise is so fast with prompt detonation that we can ignore the time passed (but not the fraction burned) and proceed to the second part.

For the second part, we try a pressure decay according to

$$
\frac{\partial F_{2}}{\partial t}=A P=A P_{k} \exp \left(-\frac{t}{\tau_{2}}\right)
$$

We know this can't really be right, because the I\&G had two growth terms, so we should really use two exponential terms. For simplicity, we press on with one term, which integrates to

$$
\mathrm{F}_{2}=\mathrm{F}_{1}+\mathrm{AP}_{\mathrm{k}} \tau_{2}\left[1-\exp \left(-\frac{\mathrm{t}}{\tau_{2}}\right)\right]
$$

With complete reaction, we have

$$
1-\mathrm{F}_{1} \approx \mathrm{AP}_{\mathrm{k}} \tau_{2}
$$

For our case, we get $\tau_{2} \approx 0.12 \mu \mathrm{s}$. With $\mathrm{U}_{\mathrm{S}}=7.5 \mathrm{~mm} / \mu \mathrm{s}$, the reaction zone, as defined to $\mathrm{F}=0.9$, becomes

$$
<\mathrm{x}_{\mathrm{r}}>\approx 2.2 \tau_{2} \mathrm{U}_{\mathrm{S}} \approx 1.9 \mathrm{~mm}
$$

which may be compared with about $2.0 \mathrm{~mm}$ from Eq. 16 .

We combine Eqs. 22 and 23 to get

$$
\mathrm{A}<\mathrm{x}_{\mathrm{r}}>=\frac{2.2 \mathrm{U}_{\mathrm{s}}\left(1-\mathrm{F}_{1}\right)}{\mathrm{P}_{\mathrm{k}}}
$$


As $A$ changes, so does everything else. The result, however, is that $A<x_{r}>$ is almost constant as seen here. This is an empirical happenstance that proves useful in visualizing the experiment.

\begin{tabular}{|c|c|c|c|c|c|c|c|c|}
\hline \multirow{2}{*}{$\begin{array}{c}\text { Coeff. } \\
\text { A in } \\
\text { AP }\end{array}$} & \multicolumn{2}{|c|}{ Product $A<x_{r}>$} & \multicolumn{2}{|c|}{ Product $\mathrm{AL}_{0}{ }^{2}$} & \multirow{2}{*}{$\begin{array}{c}\text { Meas } \\
U_{\mathrm{s}} \\
(\mathrm{mm} / \mu \mathrm{s})\end{array}$} & \multirow{2}{*}{$\begin{array}{c}\text { uncon- } \\
\text { fined } \\
\sigma\end{array}$} & \multirow{2}{*}{$\begin{array}{c}\text { distance } \\
\text { to } \mathrm{P}_{\mathrm{k}} \\
(\mathrm{mm})\end{array}$} & \multirow[b]{2}{*}{$\beta$} \\
\hline & Meas & Calc & Meas & Calc & & & & \\
\hline 2 & 20 & 42 & 61 & 60 & 6.47 & 4 & 2.2 & 0.5 \\
\hline 5 & 20 & 27 & 88 & 59 & 6.88 & 6 & 1.5 & 1.0 \\
\hline 10 & 25 & 28 & 90 & 41 & 7.22 & 9 & 1.1 & 1.0 \\
\hline 15 & 29 & 29 & 79 & 38 & 7.53 & 10 & 1.2 & 1.0 \\
\hline 20 & 35 & 28 & 65 & 35 & 7.62 & 10 & 1.1 & 1.0 \\
\hline
\end{tabular}

We take Eq. 29 from Chapter 8, which relates $\left\langle x_{e}>\right.$ to $L_{0}{ }^{2}$. Because we get $\left\langle x_{r}>\right.$ from I\&G, we define the fraction $\beta$ equal to $\left\langle x_{e}\right\rangle /\left\langle x_{r}\right\rangle$. This fraction should equal 1 for short reaction zones and become small for long zones. We obtain

$$
\mathrm{AL}_{\mathrm{o}}^{2}=\left(\frac{\beta \mathrm{R}_{\mathrm{o}}}{\sigma}\right) \mathrm{A}<\mathrm{x}_{\mathrm{r}}>
$$

Because $A<x_{r}>$ is almost constant, we expect $\mathrm{AL}_{0}{ }^{2}$ also to be nearly constant. $A$ fine point is that Eq. 26 has been multiplied by 2 . This is because our $\left\langle x_{r}\right\rangle$ is defined for $F=0.9$ whereas the equation wants the more complete zone length out to 0.99 or 4 to 5 time constants. In order match the $\mathrm{AL}_{0}{ }^{2}$ values, we find we must adjust $\beta$ in the way we expected.

It is possible that the pressure in Eq. 15 could be raised to some power. We have found that I\&G in DYNA2D does not work with fractional powers of $P$ unless $F$ terms are included. This appears to be programming problem. However, the relation

$$
\frac{\partial \mathrm{F}}{\partial \mathrm{t}}=\mathrm{BP}^{2}
$$

runs and the results are shown above in Eq. 16. It, too, can reproduce the steady state detonation curvature. Which form is correct requires a further test. However, it appears that a simplified form of I\&G may work for prompt detonation problems.

\section{References}


1. B. Hayes and C. M. Tarver, "Interpolation of Detonation Parameters from Experimental Particle Velocity Records," Proceedings Seventh Symposium (International) on Detonation. Annapolis, MD. June 16-19, 1981, p. 1029.

2. Craig Tarver, private communication, 1995.

3. Paul Urtiew, unpublished data, private commmunication, 1995.

4. F. E. Walker and R. J. Wasley, Explosivestoff 17, 9 (1969).

5. P. W. Cooper, "A New Look at the Run Distance Correlation and Its Relationship to Other NonSteady-State Phenomena," Proceedings Tenth International Detonation Symposium, Boston, MA. July 12-16, 1993 , p. 690 .

6. LASL Explosive Property Data, T. R. Gibbs and A. Popolato, ed. (University of California, Berkeley, 1980), pp. 297-379.

7. B. M. Dobratz and P. C. Crawford, LLNL Explosives Handbook, LLNL report UCRL-52997 revision 2 (1985), pp. 9-50, 9-51.

8. B. D. Trott and R. G. Jung, "Effect of Pulse Duration on the Impact Sensitivity of Solid Explosives," Proceedings Fifth Symposium (International) on Detonation, Pasadena, CA, August 1821, 1970, p. 191.

9. C. M. Tarver, R. L. Simpson and P. A. Urtiew, "Shock Initiation of an $\varepsilon-C L-20$ Estane Formulation," APS Topical Conference on Shock Compression of Condensed Mattter, Seattle, WA. August 13 - 18, 1995.

10. M. J. Murphy, R. L. Simpson, R. D. Breithaupt and C. M. Tarver, "Reactive Flow Measurements and Calculations for $\mathrm{ZrH}_{2}$-Based Composite Explosives," Proceedings Ninth Symposium (International) on Detonation, August 28-September 1, 1989, Portland, OR, vol. I, p. 525. 


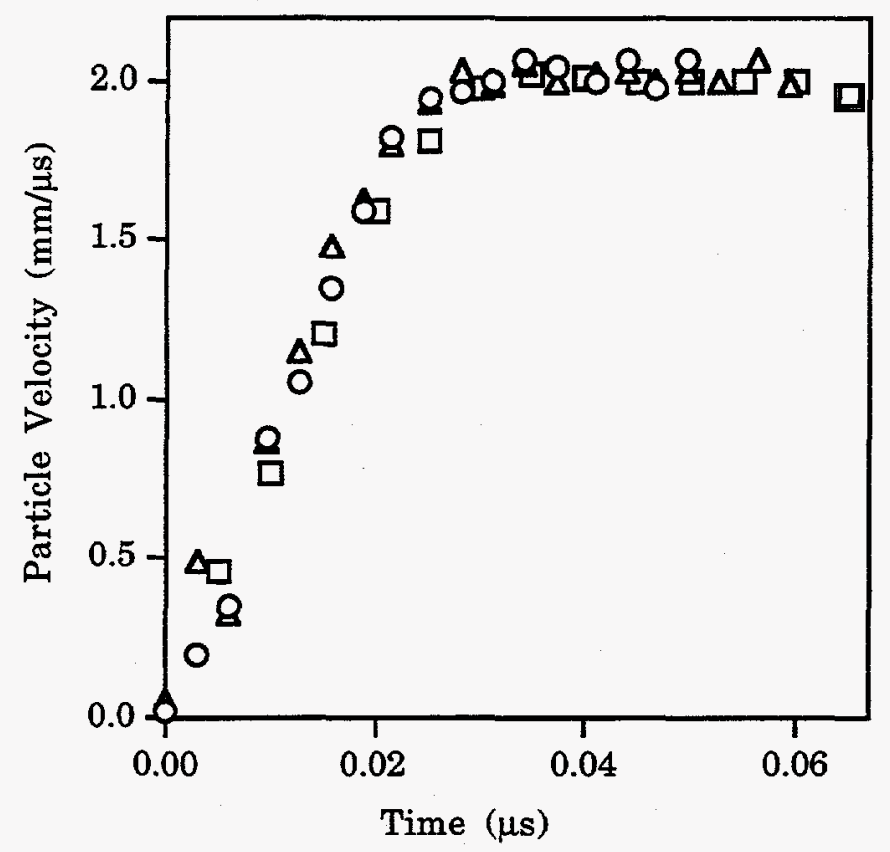

Fig. 9-1. Gauge-measured particle velocity turn-on for RX-26-AH at steady state. Three runs are overlaid. The time constant, caused by the gauge, is less than $20 \mathrm{~ns}$.

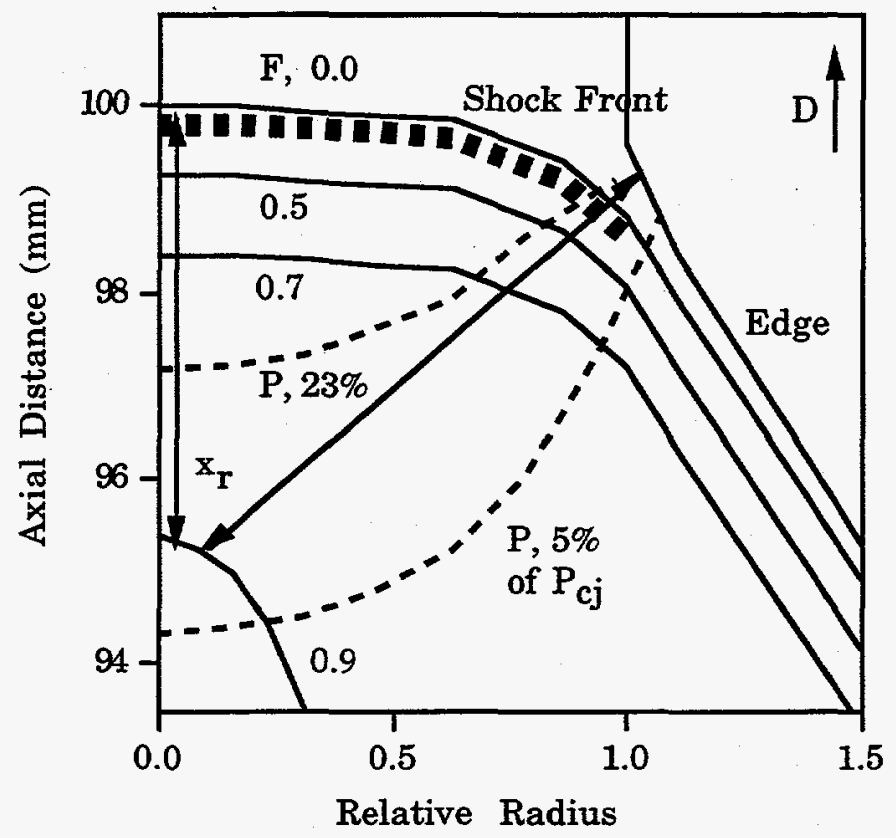

Fig. 9-2. Ignition \& Growth calculations for the composite explosive RX-25-BF/BP, showing the narrow shock front and the long reaction zone. The pressure declines rapidly behind the front but the explosive is still burning. The detonation moves upward. The solid lines are the fraction burned, $F$. The dashed lines are pressure in percent of the $0.28 \mathrm{Mb} P_{c j}$ value. The reaction zone, $x_{r}$, is shown along the axis and to the edge. 


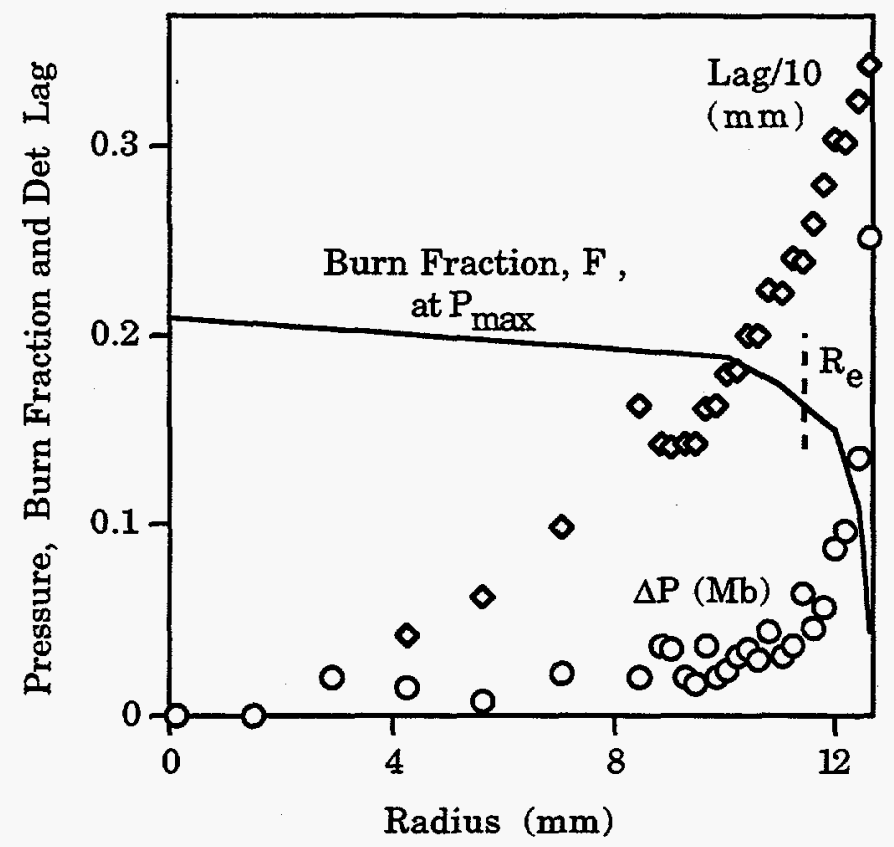

Fig. 9-3. Ignition \& Growth results for the largest $12.7 \mathrm{~mm}$ radius calculation of the composite explosive RX-25-BF/BP. All results are at steady state on the detonation front at the point of maximum pressure. The lag is divided by 10 to get it on the same scale. $R_{e}$ is the approximate edge layer thickness.

Table 9-1. Summary of particle velocity turn-on time constants for four explosives as measured directly by in-situ gauges.

\begin{tabular}{ccccc} 
Explosive & shot series & $\begin{array}{c}\text { no. in } \\
\text { series }\end{array}$ & $\tau_{\mathrm{ss}}(\mu \mathrm{s})$ & $\begin{array}{c}\mathrm{u}_{\max } \\
(\mathrm{mm} / \mu \mathrm{s})\end{array}$ \\
\hline LX-14 & 4176 & 3 & 0.021 & 2.0 \\
LX-14 & Tarver & 1 & 0.012 & 2.4 \\
LX-04 & 4204 & 3 & 0.019 & 2.15 \\
RX-26-AH & 4177 & 3 & 0.015 & 2.0 \\
LX-17 & 4158 & 3 & 0.017 & 2.1 \\
LX-17 & Tarver & 1 & 0.022 & 2.3 \\
LX-17 & misc & 4 & $\frac{0.01 \text { to } 0.04}{1.4}$ to 2.2 \\
\cline { 1 - 1 } Average & & & &
\end{tabular}


Table 9-2. Reaction zones obtained by extrapolation of run-to-detonation distances.

\begin{tabular}{|c|c|c|c|c|c|c|c|c|}
\hline & \multirow{3}{*}{$\begin{array}{c}\text { Density } \\
(\mathrm{g} / \mathrm{cc})\end{array}$} & \multicolumn{3}{|c|}{ Reaction Zone, $\mathbf{x}_{\mathbf{e}}{ }^{\circ}(\mathrm{mm})$} & \multirow{3}{*}{$\begin{array}{c}\mathrm{P}^{2} \mathrm{x} \\
\text { constant } \\
\mathrm{a}\end{array}$} & & & \multirow{3}{*}{$\begin{array}{c}\mathrm{P}_{\mathrm{cj}} \\
(\mathrm{GPa})\end{array}$} \\
\hline & & \multirow{2}{*}{$\begin{array}{l}\text { from } \\
\mathrm{P}^{2} \mathrm{x}\end{array}$} & \multirow{2}{*}{$\begin{array}{l}\text { from } \\
\log - \\
\log \end{array}$} & \multirow{2}{*}{$\begin{array}{c}\text { from } \\
\text { Inverse } \\
\text { Radius }\end{array}$} & & \multicolumn{2}{|c|}{$\log -\log$ constants } & \\
\hline & & & & & & $\mathrm{A}$ & $\mathrm{B}$ & \\
\hline PETN & 1.00 & 0.016 & 0.000 & & 0.30 & -0.39 & 0.29 & 8.5 \\
\hline PETN & 1.40 & 0.037 & 0.008 & & 2.7 & 0.14 & 0.40 & 17 \\
\hline PETN & 1.60 & 0.038 & 0.039 & & 5.9 & 0.39 & 0.50 & 25 \\
\hline PETN & 1.72 & 0.1 & 0.2 & & 16.7 & 0.65 & 0.60 & 31 \\
\hline PETN & 1.75 & 0.080 & 0.025 & & 18.1 & 0.57 & 0.41 & 30 \\
\hline Tetryl & 1.40 & 0.2 & 1 & & 14.9 & 0.84 & 0.99 & 19 \\
\hline PBX-9404 & 1.72 & 0.2 & 0.8 & & 39.5 & 0.96 & 0.71 & 29 \\
\hline Tetryl & 1.50 & 0.2 & 1 & & 14.7 & 0.75 & 0.81 & 16 \\
\hline $\mathrm{LX}-19$ & 1.94 & 0.3 & & & 115 & & & 41 \\
\hline Tetryl & 1.70 & 0.3 & 0.1 & & 47.1 & 0.79 & 0.42 & 26 \\
\hline XTX-8003 & 1.53 & 0.3 & 0.2 & 0.01 & 43.9 & 0.80 & 0.46 & 24 \\
\hline PBX-9404 & 1.84 & 0.3 & 0.9 & & 112 & 1.12 & 0.67 & 36 \\
\hline PBX-9501 & 1.83 & 0.4 & 0.7 & 0.25 & 113 & 1.10 & 0.59 & 35 \\
\hline HMX & 1.89 & 0.4 & 0.8 & 0.25 & 178 & 1.18 & 0.59 & 40 . \\
\hline LX-04 & 1.86 & 1 & 2 & 0.25 & 113 & 1.34 & 0.66 & 30 \\
\hline PBX-9501 & 1.84 & 0.5 & 0.5 & & 156 & 1.10 & 0.51 & 35 \\
\hline PBX-9011-06 & 1.79 & 1 & 1 & & 176 & 1.18 & 0.66 & 32 \\
\hline Comp B & 1.72 & 1 & 0.9 & 0.2 & 294 & 0.99 & 0.77 & 30 \\
\hline TATB, superfine & 1.81 & 3 & 2 & & 425 & 1.31 & 0.43 & 26 \\
\hline TATB, micronizd & 1.81 & 5 & 4 & & 794 & 1.41 & 0.38 & 26 \\
\hline TATB & 1.88 & 6 & 4 & 2 & 943 & 1.42 & 0.40 & 26 \\
\hline PBX-9502 & 1.90 & 8 & 3 & 2 & 1391 & 1.39 & 0.31 & 27 \\
\hline Baratol & 2.61 & 12 & 7 & 5 & 567 & 1.24 & 0.34 & 14 \\
\hline NQ, commercial & 1.69 & 13 & 13 & & 1909 & 1.51 & 0.26 & 24 \\
\hline TNT, cast & 1.64 & 18 & 10 & 5 & 1605 & 1.40 & 0.32 & 19 \\
\hline NQ, large grain & 1.70 & 22 & 10 & & 3230 & 1.44 & 0.15 & 24 \\
\hline PBX-9407 & 1.60 & & 0.060 & & & 0.57 & 0.49 & 29 \\
\hline TNT, pressed & 1.63 & & 1 & 1 & & 1.08 & 0.39 & 19 \\
\hline Tetryl & 1.30 & & 3 & & & 0.87 & 1.11 & 12 \\
\hline LX-17 & 1.90 & & 5 & 2 & & 1.49 & 0.57 & 27 \\
\hline
\end{tabular}





\section{Chapter 10. Temperature and Thermochemical Codes}

\section{Direct Temperature Measurements (by Choong-Shik Yoo and Neil Holmes)}

Recent experiments on the LLNL two-stage gas gun have provided direct and modern detonation temperature measurements suitable for use as a calibration C-J temperature in CHEETAH in the future. ${ }^{1}$ The gun pushes the detonating explosive in front of it, creating a microsecond-long plateau of constant pressure. Using transparent explosives, the emitted optical radiation is measured between 350 and $700 \mathrm{~nm}$ with time and spectral resolution of $1 \mathrm{~ns}$ and $2 \mathrm{~nm}$. respectively. An aluminum sabot is fired into an aluminum baseplate behind which is the explosive. At the other end of the explosive is a sapphire window through which the radiation is observed. The experiment was one-dimensional for the time it took for the edge effects to move down the radius, $R$, to the center at the speed of sound in the reacted products, which is roughly 3D/4. The 1-D time is

$$
1-\mathrm{D} \text { time } \approx 4 \mathrm{R} / 3 \mathrm{D}
$$

For NM, TNM and PETN, this time was $2.0,2.0$ and $1.5 \mu \mathrm{s}$.

A well-behaved example is shown in Fig. 10-1 for nitromethane. The intent is to hit the sample above the detonation pressure and gradually work down until the sample fails to detonate. The lowest detonation pressure should be close to being a C-J point. Table 10-1 lists the measured plateau temperatures, which decline slightly as the flyer velocity is decreased and the pressure drops. The pressure was calculated first assuming an unreacted Hugoniot using impedance matching, then using a reacted JWL in the LLNL 1-D hydrocode. The cylinder JWL from Chapter 3 was used and both sets of pressures are given in Table 10-1. The reacted values are better for the cases where the NM detonated, so that $12.8 \mathrm{GPa}$ is a good estimate of the detonation pressure. We see that it takes longer for it to reach the plateau here than at the higher pressures. The lowest shot did not detonate and the impedance pressure of $8.8 \mathrm{GPa}$ is probably the best estimate. The plateau state is not a pure adiabat state but contains energy given by the sabot. Hence, the mental jump from this plateau state to estimating the C-J temperature requires that the measured temperatures be largely constant. We summarize our best estimates of the measured "C-J" temperature for three explosives with the calculated C-J temperatures from CHEETAH and CHEQ: 


\begin{tabular}{cccc} 
Expl. & Measd. & CHEETAH & CHEQ \\
\hline NM & 3900 & 3600 & 3700 \\
TNM & 2920 & 2700 & 2300 \\
PETN & 4200 & 4300 & 4400
\end{tabular}

All shots show a spike caused by the initial impact of the sabot. One NM shot showed a hint of a pre-spike initiation, which was larger for TNM. The PETN single crystal shots are shown in Fig. 10-2. At the left are two shots on the insensitive $<110>$ crystal direction. The $34 \mathrm{GPa}$ shot, just above the $31 \mathrm{GPa}$ detonation point, caused detonation but the lower pressure shot failed. At the right are two underdriven shots on the sensitive $\langle 100\rangle$ crystal direction. The underinitiated $\langle 100\rangle$ shots show a droop in the temperature following the spike, which represents the initiation period. Once detonation begins, it is very fast. Oddly enough, the underinitiated $<110>$ shot shows no temperature droop; the process just fizzles out.

The TNM JWL was taken from Chapter 3. The $1.766 \mathrm{~g} / \mathrm{cc}$ PETN JWL was modified to give this estimated single crystal, theoretical density JWL:

\begin{tabular}{lc|lc}
$\rho_{\mathrm{o}}(\mathrm{g} / \mathrm{cc})$ & 1.78 & $\omega$ & 0.29 \\
$\mathrm{~A}(\mathrm{Mb})$ & 6.43609 & $\mathrm{E}_{\mathrm{o}}(\mathrm{Mb})$ & 0.1129 \\
$\mathrm{~B}(\mathrm{Mb})$ & 0.20655 & $\Gamma_{\mathrm{j}}+1$ & 3.69 \\
$\mathrm{C}(\mathrm{Mb})$ & 0.015421 & $\mathrm{D}(\mathrm{cm} / \mathrm{s})$ & 0.833 \\
$\mathrm{R}_{1}$ & 4.50 & $\mathrm{P}_{\mathrm{j}}(\mathrm{Mb})$ & 0.334 \\
$\mathrm{R}_{2}$ & 1.50 & $\mathrm{v}_{\mathrm{j}}$ & 0.729
\end{tabular}

\section{Semi-Empirical Calibration of the CHEQ Intermolecular Potentials (by Matt van Thiel and Francis Ree)}

All explosive prediction begins with a thermochemical code. The two mentioned often in this report are CHEETAH and CHEQ. CHEETAH, the descendent of TIGER, is well known as an empirical code. It describes pressure in terms of an analytic compressibility function, which contains four global coefficients plus a co-volume for each reaction product species. These coefficients have been brute-force calibrated to detonation pressures, cylinder test energies and total detonation energies. Less well known is that CHEQ also has an empirical heart, created by considerable toil. ${ }^{2}$ Despite its wealth of scientific detail, the ab-initio science needed to make detonation calculations converge to the correct answers, has not yet been found. 
In CHEQ, the intermolecular potentials are used for the gas-phase components are of the exponential repulsive and inverse sixth power attractive form, $3-4$

$$
\phi=\frac{\varepsilon}{\alpha-6}\left\{6 \exp \left[\alpha\left(1-\mathrm{r} / \mathrm{r}^{*}\right)\right]-\alpha\left(\mathrm{r}^{*} / \mathrm{r}\right)^{6}\right\}
$$

This so called (exp-6) potential which has been the preferred form for high pressure work for some time. For most systems we use a constant $\varepsilon$. A more general form of the pair parameter $\varepsilon$ is

$$
\varepsilon_{i i}=\varepsilon_{i i}^{\prime}(1+\lambda T)
$$

with $\lambda_{\mathrm{ii}} \neq 0$ for polar molecules. The reason is the decreased accuracy of the spherically symmetric potential at low temperatures, where dipole-dipole alignment becomes a factor. The like-pair parameter $\varepsilon_{\mathrm{ii}}$ can be found by pressure experiments on the specific substance.

In a mixture, there will be more unlike-pair parameters than like. But they can be expressed as

$$
\begin{aligned}
& \varepsilon_{i j}=k_{i j} \sqrt{\varepsilon_{i i} \varepsilon_{j j}} \\
& r_{i j}^{*}=l_{i j}\left(r_{i i}^{*}+r_{i j}^{*}\right) / 2 \\
& \alpha_{i j}=m_{i j} \sqrt{\alpha_{i j} \alpha_{j j}}
\end{aligned}
$$

where ii and jj refer to like molecules and ij to two unlike molecules. The unlike-pair parameters are given in terms of the like-pair constants $\mathrm{r}_{\mathrm{ij}}^{*}$, $\varepsilon_{\mathrm{ii}}$ and $\alpha_{\mathrm{ii}}$ and small multiplicative constants $\mathrm{k}_{\mathrm{ij}}$, $\mathrm{l}_{\mathrm{ij}}$, $m_{\mathrm{ij}}$, which can be fixed if appropriate theoretical or experimental data are available. Otherwise, we set $\mathrm{k}_{\mathrm{ij}}=\mathrm{l}_{\mathrm{ij}}=\mathrm{m}_{\mathrm{ij}}=1$, commonly referred to as the Lorentz-Berthelot rule. This rule allows us to proceed even though the data we need to establish the unlike-pair parameters is not available.

We assume that mixtures of chemical species interacting with exp-6 potentials can be represented by a hypothetical one-component system with exp- 6 parameters that are composition $\left(x_{i}\right)$-weighted averages of the parameters of the component pair potentials, as follows: 


$$
\begin{aligned}
& \left(r^{*}\right)^{3}=\sum x_{i} x_{j}\left(r_{i j}^{*}\right)^{3}, \\
& \varepsilon=\sum x_{i} x_{j} \varepsilon_{i j}\left(r_{i j}^{*} / r^{*}\right)^{3}, \\
& \alpha=\sum x_{i} x_{j} \alpha_{i j} \frac{\varepsilon_{i j}\left(r_{i j}^{*}\right)^{3}}{\varepsilon\left(r^{*}\right)^{3}} .
\end{aligned}
$$

The pair parameters are obtained from shock wave experiments. CHEQ, for example calculates the shock wave velocity for $\mathrm{N}_{2}$; then the result is compared with the actual experiment. Pure samples do not stay that way. Because nitrogen disocciates in the shock wave, we have:

$$
\mathrm{N}_{2}<--->2 \mathrm{~N}
$$

which means that the parameters for $\left(\mathrm{N}_{2}-\mathrm{N}_{2}\right),\left(\mathrm{N}_{2}-\mathrm{N}\right)$ and $(\mathrm{N}-\mathrm{N})$ are involved. The same is true for oxygen. $\mathrm{CO}_{2}$ comes apart into $\mathrm{CO}$ and $\mathrm{O}$. The atomic oxygen can form $\mathrm{O}_{2}$, so that 10 parameters are involved, not counting the possible formation of a solid carbon phase.

It would seem easy to get the unlike parameters by mixing the components, eg. $\mathrm{N}_{2}$ and $\mathrm{H}_{2} \mathrm{O}$. But these two gases are immiscible unless about $1000 \mathrm{~K}$ is applied. So a mixing chamber of high temperature and high pressure is needed as a starter, which makes such experiments expensive.

With unlike pair experiments generally unavailable, the next step is to find explosives that come apart into simple unlike pairs. This is like a mixture experiment but with all the energy of detonation added. There are only two such explosives. $\mathrm{RX}-23-\mathrm{AB}$ is a liquid mixture of hydrazine nitrate, hydrazine and water, which comes apart mainly into water and nitrogen.

$$
\mathrm{H}_{3.1} \mathrm{NO}_{1.5} \rightarrow 3 / 2 \mathrm{H}_{2} \mathrm{O}+1 / 2 \mathrm{~N}_{2}
$$

HNB is a highly oxidized explosive that spilts into carbon dioxide and nitrogen.

$$
\mathrm{C}_{6} \mathrm{~N}_{6} \mathrm{O}_{12} \rightarrow 6 \mathrm{CO}_{2}+3 \mathrm{~N}_{2} \text {. }
$$

Calorimetry confirms that these explosives form mostly the expected ingredients. 5 The RX-23 series was made of unpleasant liquids, whose mixture properties were somehat uncertain, despite all the work done on them. HNB, the most powerful explosive ever made, was hyper-sensitive and the 
result of a unique synthesis at China Lake. So neither of these explosives constitutes a handy ongoing standard and the cylinder shots were made years ago.

What remains are the explosives that don't come apart into handy pairs. BTF, for example, looks like a natural winner with

$$
\mathrm{C}_{6} \mathrm{~N}_{6} \mathrm{O}_{6}-- \text { want---> } 3 \mathrm{~N}_{2}+6 \mathrm{CO} \text {. }
$$

However, the $\mathrm{C}+\mathrm{CO}_{2}<--->2 \mathrm{CO}$ equilibrium undoes this, so that calorimetry finds $1 / 3 \mathrm{CO}_{2}$ and 2/3CO, along with considerable precipitated carbon.

Another explosive used in calibration is PETN, which is, at least, easy to get. PETN is a carbon dioxide-water generator. What we want is

$$
\mathrm{C}_{5} \mathrm{H}_{8} \mathrm{~N}_{4} \mathrm{O}_{12}-\text {-want---> } 5 \mathrm{CO}_{2}+4 \mathrm{H}_{2} \mathrm{O}+2 \mathrm{~N}_{2} \text {. }
$$

PETN is two oxygens short of being able to handle both the carbon and the hydrogen at once. Also; the presence of nitrogen adds an additional complication. The calorimetry for PETN shoes 3.4 mols/mol of $\mathrm{CO}_{2}, 1.7 \mathrm{CO}, 3.5 \mathrm{H}_{2} \mathrm{O}, 2.0 \mathrm{~N}_{2}$ and $0.4 \mathrm{H}_{2}$ (but no solid carbon). 5 So PETN is beginning to degrade down into the complicated mixture we were hoping the code would explain. The addition of solid carbon, present in most reacted oxygen-poor experiments, complicates the results even more.

Tables 10-2 and 10-3 summarize the results of the authors' self-consistent approach, where they first established like-pair parameters from all available Hugoniot data, then moved on to estimate unlike-pair parameters as best they could using explosive results. This considerable labor has brought the computation of detonation at the intermolecular potential level to the point where it now exists. It is also important that research continue into the measurement of the unlike pair potentials. The importance of continuing to collect the needed semi-empirical input is perhaps not fully appreciated.

\section{Carbon not the Reason for the Freeze (by Francis Ree, W. J. Pitz, and Matt van Thiel)}

In Chapter 2, we mentioned the freeze put into every thermochemcial code run. This consists of setting the reaction products as fixed at some temperature as the explosive moves down the adiabat. In CHEQ, the traditional $1800 \mathrm{~K}$ is used. In CHEETAH, a value of $2145 \mathrm{~K}$ was selected as a result of optimizing the various coefficients. Neither temperature is a hard value, but some freeze 
must be done in order to match the calorimetric total energies of detonation and the overabundance of $\mathrm{CO}$ found in the calorimeter product recovery.

The first idea was that we are not modeling the solid carbon properly: that some fraction of solidified carbon is out of equilibrium with the rest of detonation products, i.e., effectively removed from chemical reactions. Removal of such nonequilibrium carbon shifts the equilibrium composition of remaining species by the law of mass action to account for the observed overabundance of CO.

To explain why this is not the case, consider the key reaction,

$$
\mathrm{C}(\mathrm{s})+\mathrm{CO}_{2}<-\cdots>2 \mathrm{CO}
$$

between solid carbon $\mathrm{C}(\mathrm{s})$ with gaseous detonation products at low pressure $(\mathrm{P})$ and low temperature (T). Let us assume that $\mathrm{C}(\mathrm{s})$ is in equilibrium with $\mathrm{CO}_{2}$ and $\mathrm{CO}$, with equilibrium constant $\mathrm{K}$ given by

$$
\mathrm{K}=[\mathrm{CO}]^{2} /[\mathrm{C}(\mathrm{s})]\left[\mathrm{CO}_{2}\right]
$$

Let us permanently remove a small $(\Delta)$ amount of solid carbon. The removal will shift the equilibrium compositions of remaining species including that $[C(s)-\Delta]$ of the solid carbon clusters. If we define $\Delta^{\prime}$ to be a change in concentration of the remaining carbon clusters, we wish to know if $\Delta^{\prime}$ is positive or negative. The mass conservation requires

$$
\begin{aligned}
& {[\mathrm{C}(\mathrm{s})]-->[\mathrm{C}(\mathrm{s})]-\Delta+\Delta^{\prime},} \\
& {\left[\mathrm{CO}_{2}\right]-->\left[\mathrm{CO}_{2}\right]+\Delta^{\prime},} \\
& {[\mathrm{CO}]-\rightarrow[\mathrm{CO}]-2 \Delta^{\prime},}
\end{aligned}
$$

and the corresponding equilibrium constant $\mathrm{K}^{\prime}$ is given by

$$
\mathrm{K}^{\prime}=\left([\mathrm{CO}]-2 \Delta^{\prime}\right)^{2} /\left([\mathrm{C}(\mathrm{s})]-\Delta+\Delta^{\prime}\right)\left(\left[\mathrm{CO}_{2}\right]+\Delta^{\prime}\right)
$$

At fixed pressure and temperature, $K=K^{\prime}$. This condition is satisfied only if $\Delta^{\prime}$ is positive. Namely, removal of any $\Delta$ amount of carbon in a nonequilibrium fashion increases the amount of $\mathrm{CO}_{2}$ and reduces the $\mathrm{CO}$ concentration. This decrease in $\mathrm{CO}$ is inconsistent with the calorimetry data. 
4. Temperature Quenching is the Reason for the Freeze (by Francis Ree, W. J. Pitz, Matt van Thiel)

We now consider the detonation of $1.74 \mathrm{~g} / \mathrm{cc}$ PETN. ${ }^{6}$ The CHEQ code predicts the state corresponding to the adiabatic expansion to $\mathrm{v}=370$ to be at $791 \mathrm{~K}$ and $9.3 \mathrm{~atm}$. At this point, the products hit the wall of the bomb. The kinetic energy is turned into internal energy. Using the CHEQ heat capacity, we find that the products jump from 789 to $2078 \mathrm{~K}$ upon this collision. It takes less than $1 \mathrm{~ms}$ to reach this point, which is the real start of our story. CHEQ prdicts the products, in $\mathrm{mol} / \mathrm{mol}$, to be $1.63 \mathrm{CO}, 3.37 \mathrm{CO}_{2}, 3.63 \mathrm{H}_{2} \mathrm{O}, 2.00 \mathrm{~N}_{2}$, and $0.37 \mathrm{H}_{2}$.

We next used the HCT code, ${ }^{7}$ developed for LLNL combustion research to integrate the species conservation equations as a function of time. The gaseous mixture in the calorimetry bomb was treated as homogenous, so that no spatial variation in temperature, pressure and composition is considered. The chemical kinetic mechanism used included $\mathrm{CO}, \mathrm{CO}_{2}, \mathrm{H}_{2} \mathrm{O}, \mathrm{N}_{2}, \mathrm{H}_{2}, \mathrm{CH}_{4}, \mathrm{O}_{2}$, and 152 additional gaseous species and 1002 chemical reactions with rate constants expressed in an Arrhenius form. ${ }^{8}$ The present reaction mechanism neglects reactions leading to the solidification of carbon, as its coagulation rate is presently not well-known.

The calculations allowed a convective heat transfer between detonation products within the calorimetry bomb at temperature $\mathrm{T}$ and the water reservoir at temperature $\mathrm{T}_{0}$ with the total heat flow q given by

$$
q=h A\left(T-T_{0}\right)
$$

where $A$ is the surface area of the bomb and $h$ is the convective heat transfer coefficient. For the free convection of air, $\mathrm{h}$ is 6 to $30 \mathrm{watts} / \mathrm{m}^{2} \cdot \mathrm{K}$ and, for the forced convection, it is 30 to $300 \mathrm{watts} / \mathrm{m}^{2} \cdot \mathrm{K} .^{9}$ The present calculation used $\mathrm{h}=30 \mathrm{watt} / \mathrm{m}^{2} \cdot \mathrm{K}$.

The composition of $\mathrm{CO}$ slightly decreases during the first 4 seconds or so by the overall "water-gas" reaction,

$$
\mathrm{CO}+\mathrm{H}_{2} \mathrm{O} \longrightarrow \mathrm{H}_{2}+\mathrm{CO}_{2}
$$

to produce $\mathrm{H}_{2}$ and $\mathrm{CO}_{2}$. Eq. 18 is really a pair of chain reactions 


$$
\begin{aligned}
& \mathrm{CO}+\mathrm{OH}-\cdots->\mathrm{H}+\mathrm{CO}_{2}, \\
& \mathrm{H}+\mathrm{H}_{2} \mathrm{O}-\cdots>\mathrm{OH}+\mathrm{H}_{2} .
\end{aligned}
$$

The forward rate constant for Eq. 19 is $1.5 \times 10^{7} \mathrm{~T}^{1.3} \exp (+7.65 \mathrm{cal} / \mathrm{RT}) \mathrm{cm}^{3} / \mathrm{mole.sec}$ and has no kinetic barrier, while that for Eq. 20 is $9.3 \times 10^{8} \mathrm{~T}^{1.51} \exp (-18,580 \mathrm{cal} / \mathrm{RT}) \mathrm{cm}^{3} / \mathrm{mole.sec}$ which will considerably slow down the reaction for temperatures below $1000 \mathrm{~K}$. The rate for Eq. 20 was obtained from the reverse rate and the principle of detailed balancing.

Table 10-4 shows that the composition of chemical species remains nearly the same after 4 sec. The convective heat loss to the water reservoir causes a rapid cooling in temperature to $1350 \mathrm{~K}$ below which the rate constant for Eq. 18 becomes very small. At later time ( $>4 \mathrm{sec}$.), the concentration of $\mathrm{CO}$ still changes but slowly and in very small amount ( $\leq 0.02$ mole) until about 7 sec., or until the system cools down to about $1000 \mathrm{~K}$. So, for all practical purposes, $\mathrm{CO}$ can be considered to be effectively frozen (i.e., quenched) below an "apparent" freeze-out temperature of $1350 \mathrm{~K}$.

Incidentally, Table 10-4 shows that the calculation matches better with experiment at an earlier time close the initial conditions we started. It suggests that the actual freeze-out occurs at higher temperature than the prediction $(1350 \mathrm{~K})$.

Had freezing not occured, and equilbrium chemistry continued, the "water-gas" reaction would have continued down to $900 \mathrm{~K}$. Then, more $\mathrm{CO}$ would have been used up between 900 and 750 $\mathrm{K}$ in the reaction

$$
2 \mathrm{CO}+2 \mathrm{H}_{2}-\mathrm{CH}_{4}+\mathrm{CO}_{2}
$$

To form $\mathrm{CH}_{4}$ from $\mathrm{CO}$ requires the breaking of a double bond. This process requires reaction steps with very high activation energies ( 50 to $100 \mathrm{kcal} / \mathrm{mole})$. These steps proceed very slowly at temperatures below $1000 \mathrm{~K}$. Consequently, even though the formation of $\mathrm{CH}_{4}$ is favored thermodynamically, the reactants have insufficient energy to overcome the energy barriers to proceed to methane.

Finally, below $750 \mathrm{~K}$, we would have lost $\mathrm{CO}_{2}$ in the reaction

$$
2 \mathrm{CO}_{2}+\mathrm{CH}_{4}+2 \mathrm{H}_{2}-\cdots \text { graphite }+4 \mathrm{H}_{2} \mathrm{O}
$$


which does not affect the CO.

The convective heat flow was altered to different rates but the overall results were not affected.

\section{Variability of the Freeze?}

In the previous section, we saw that the freezing occurs when the declining temperature turns off the $\mathrm{CO}+\mathrm{H}_{2} \mathrm{O}$ reaction. The temperature decline may depend on the dimensions of the system. The calorimeter samples were typically $12.7 \mathrm{~mm}$ in diameter and $114 \mathrm{~mm}$ long. The gold wall thickness was also about $13 \mathrm{~mm}$ as were the thicknesses of the end caps, one of which held the detonator. 5 . Thus, the calorimeter samples were similiar to a 1-inch cylinder but with heavier confinement, so that applying the calorimeter-derived $1800 \mathrm{~K}$ freeze to the Cylinder Test seemed reasonable.

The test that was not run was to sample the products as a function of the wall thickness. Would a thin-wall gold wall have resulted in freezing at a higher temperature? Similarly, would a larger charge keep burning longer? That there is a significant difference is shown in Table 10-5, where $1800 \mathrm{~K}$ freeze and equilibrium results for the JWL coefficients $\omega$ and $E_{0}(\infty)$ are shown from CHEQ calculations.

The freeze keeps the explosive from totally burning. It is not like the size effect, where the inhibition occurs at the start of the burn. Here, it occurs at the end, so that it is similiar but is the reverse of "afterburning", where air diffuses in at late time and adds energy. Further studies of the explosive size and the $\mathrm{CO}$ formation should undertaken in the future.

\section{References}

1. C. S. Yoo and N. C. Holmes, "Shock Initiation of Nitromethane," in the proceedings of the Joint Conference of High Pressure Technology and the APS Shock Compression of Condensed Matter, Colorado Springs, CO, June 28-July 2, 1993. To be presented at the APS Shock Compression conference of 1995 at Seattle.

2. M. van Thiel, F. H. Ree and L. C. Haselman, Accurate Determination of the Pair Potentials for a $\underline{\mathrm{C}}_{\mathrm{W}} \underline{\mathrm{H}}_{\mathrm{X}} \underline{\mathrm{N}}_{\mathrm{Y}} \mathrm{O}_{\mathrm{Z}}$ System of Molecules: a Semiempirical Method, LLNL Report UCRL-ID-120096 (1995).

3. F. H. Ree, "Postdetonation Behavior of Condensed High Explosives by Modern Methods of Statistical Mechanics," Proceedings Seventh Symposium (International) on Detonation, Annapolis. MD. June 16-19, 1981, p. 646.

4. F. H. Ree, J. Chem. Phys. 81, 1251 (1984). 
5. D. L. Ornellas, Calorimetric Determinations of the Heat and Products of Detonation for Explosives: October 1961 to April 1982, LLNL Report UCRL-52821 (1982).

6. F. H. Ree, W. J. Pitz, M. Van Thiel and P. C. Souers, Overabundance of Carbon Monoxide in Calorimetry Tests, LLNL report UCRL-JC-121205 (1995); submitted J. Phys. Chem.

7. C. M. Lund (revised by L. L. Chase), HCT: A General Computer Program for Calculating TimeDependent Phenomena Involving One-Dimensional Hydrodynamics, Transport, and Detailed Chemical Kinetics, Lawrence Livermore National Laboratory, Livermore, CA, UCRL-52504 (1995).

8. R. A. Yetter, F. L. Dryer, and H. Rabitz, "A Comprehensive Reaction Mechanism for the Carbon Monoxide/Hydrogen/Oxygen Kinetics", Combustion, Science and Teehnology 7997 (1991).

9. F. Kreith, Principles of Heat Transfer (Intext Educational Pub., New York, 1973), 3rd edition, p. 14.

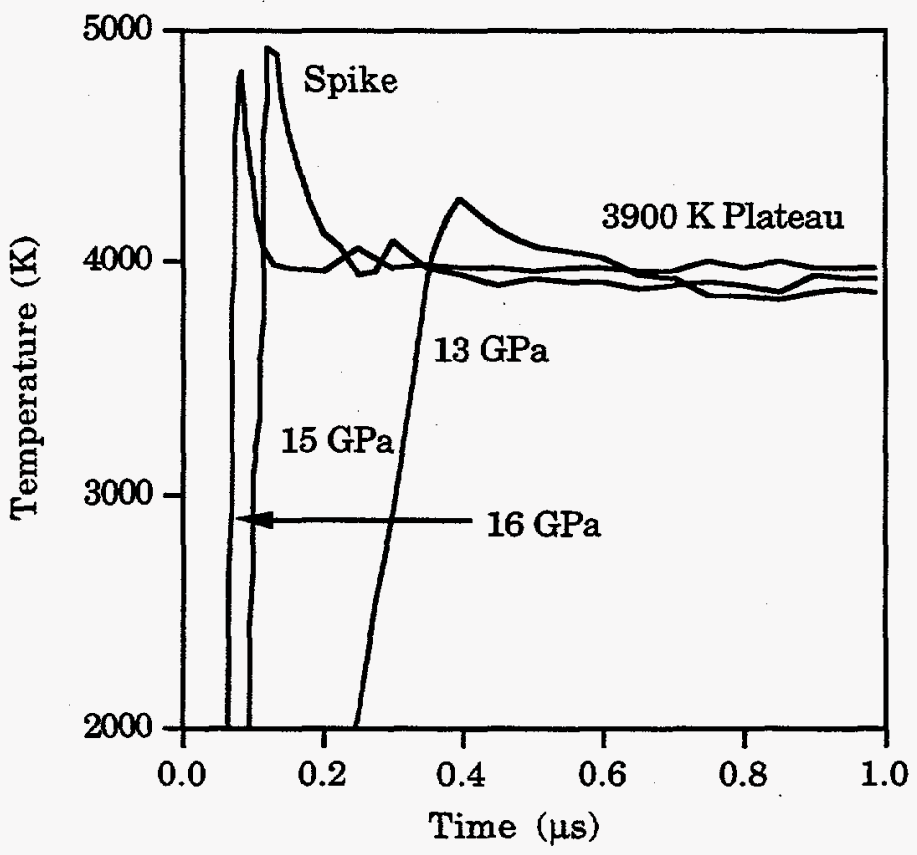

Fig. 10-1. Temperatures of nitromethane at three pressures just above the detonation point. The level microsecond-long plateau is the place to measure. The lowest pressure at which detonation occurs gives the best detonation temperature. 

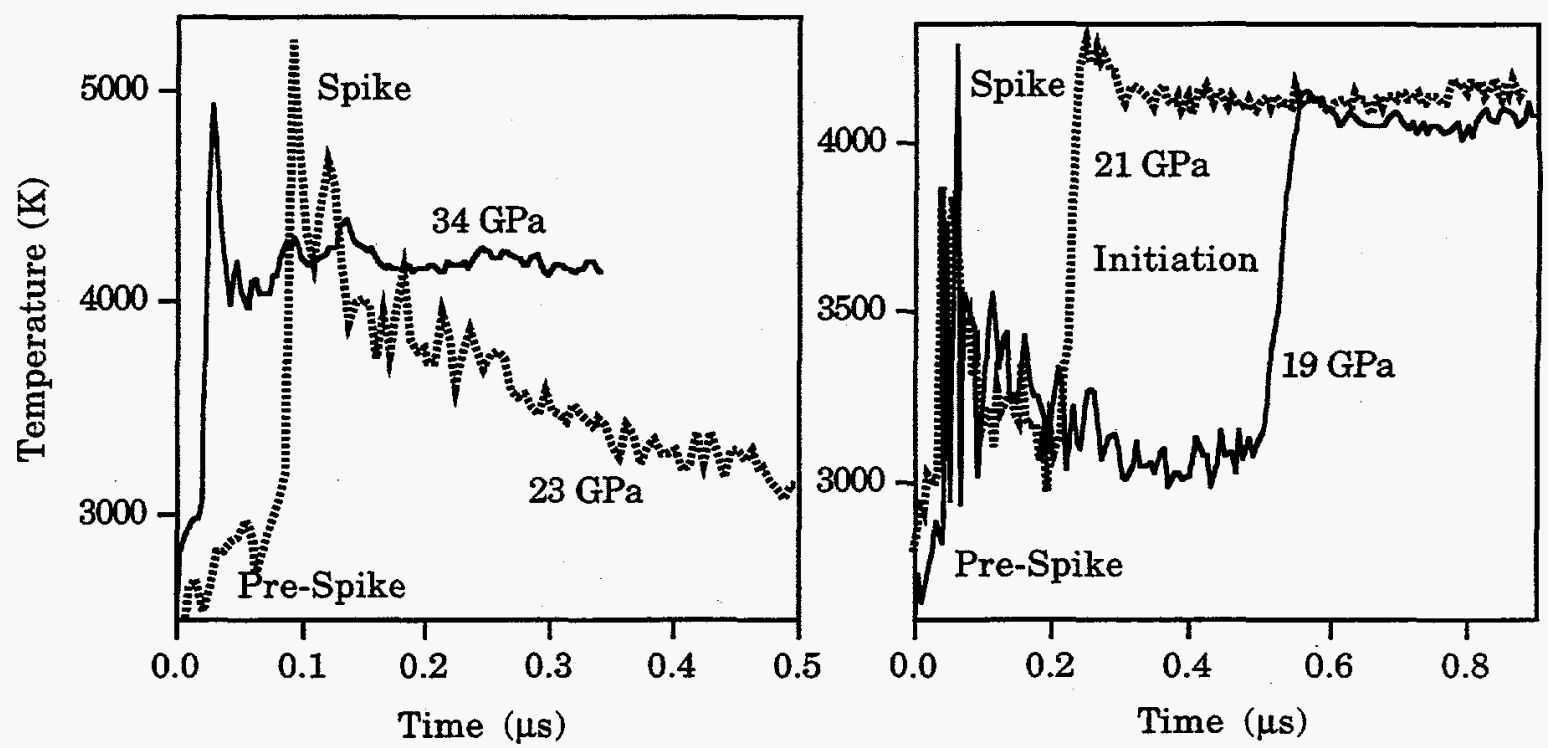

Fig. 10-2. Measured temperatures ofr PETN single crystals for left) the insensitive $\langle 110\rangle$ direction, and right) the sensitive $\langle 100\rangle$ direction. The $23 \mathrm{GPa}$ shot at the left did not detonate. The three others all gave compabable detonation temperatures. The two shots at the right are underdriven and initiate to detonation.

Table 10-1. Summary of the Yoo temperature experiments. The error bars are precision.

\begin{tabular}{ccccccc}
$\begin{array}{c}\text { Shot } \\
\text { No. }\end{array}$ & $\begin{array}{c}\text { Sabot } \\
\text { Velocity } \\
(\mathrm{mm} / \mathrm{\mu s})\end{array}$ & $\begin{array}{c}\text { Explosive } \\
\text { Thickness } \\
(\mathrm{mm})\end{array}$ & $\begin{array}{c}\text { Explosive Pressure }(\mathrm{Mb}) \\
\text { Unreacted } \\
\text { Impedance }\end{array}$ & $\begin{array}{c}\text { Reacted } \\
\text { Hydrocode }\end{array}$ & $\begin{array}{c}\text { Plateau } \\
\text { Temp } \\
(\mathrm{K})\end{array}$ & $\begin{array}{c}\text { Detonation } \\
\text { Temp }\end{array}$ \\
\hline nm2 & 3.11 & 6.00 & 0.146 & 0.159 & $3970 \pm 20$ & \\
nm1 & 2.88 & 6.00 & 0.129 & 0.146 & $3900 \pm 20$ & \\
nm3 & 2.55 & 5.98 & 0.107 & 0.128 & $3890 \pm 40$ & 3900 \\
nm4 & 2.24 & 6.00 & 0.088 & 0.112 & No detonation & \\
tnm5 & 2.88 & 6.03 & 0.193 & 0.194 & $3180 \pm 50$ & \\
tnm4 & 2.58 & 6.09 & 0.168 & 0.172 & $2930 \pm 30$ & \\
tnm1 & 2.31 & 6.03 & 0.148 & 0.154 & $2810 \pm 80$ & \\
tnm3 & 2.15 & 7.97 & 0.136 & 0.143 & $3030 \pm 140$ & \\
tnm2 & 2.06 & 7.96 & 0.129 & 0.137 & $2920 \pm 120$ & 2920 \\
tnm6 & 1.66 & 7.99 & 0.100 & 0.112 & No detonation & \\
petn3 & 4.00 & 3.20 & 0.313 & 0.345 & $4200 \pm 50$ & 4200 \\
petn4 & 3.21 & 3.17 & 0.228 & 0.273 & No detonation & \\
petn2 & 2.40 & 7.39 & 0.152 & 0.207 & $4140 \pm 40$ & \\
petn1 & 2.18 & 7.41 & 0.133 & 0.191 & $4070 \pm 40$ &
\end{tabular}

Sample Radius: $9.5 \mathrm{~mm}$; Sabot Thickness $4 \mathrm{~mm}$; Baseplate and sapphire window thicknesses $2 \mathrm{~mm}$. 
Table 10-2. Summary of like-pair exponential-6 coefficients. sources. The asterisk indicates the constants selected as best.

\begin{tabular}{lcccc} 
Material & $\varepsilon_{\mathrm{ii}} / \mathrm{k} \mathrm{K}$ & $\mathrm{r}_{\mathrm{ii}}{ }^{*}(\AA)$ & $\alpha_{\mathrm{ii}}$ & $\lambda_{\mathrm{ii}}(\mathrm{K})$ \\
\hline $\mathrm{H}_{2} \mathrm{O}$ & 356 & 3.06 & 13 & 996 \\
$\mathrm{H}_{2} \mathrm{O}^{*}$ & 300 & 3.06 & 13.1 & 1290 \\
$\mathrm{~N}_{2}$ & 101.9 & 4.09 & 13.2 & 0 \\
$\mathrm{~N}_{2}$ & 101.9 & 4.15 & 13.2 & 0 \\
$\mathrm{~N}_{2}{ }^{*}$ & 293 & 3.91 & 11.5 & 0 \\
$\mathrm{~N}$ & 600 & 2.39 & 10 & 0 \\
$\mathrm{~N}^{*}$ & 600 & 2.47 & 10 & 0 \\
$\mathrm{CO}_{2}{ }^{*}$ & 245.6 & 4.28 & 13 & 0 \\
$\mathrm{CO}_{2}$, & 245.6 & 4.2 & 13.5 & 0 \\
$\mathrm{CO}_{2}$, & 245.6 & 4.17 & 13.5 & 0 \\
$\mathrm{CO}_{2}$ & 245.6 & 4.17 & 13 & 0 \\
$\mathrm{CO}$ & 108.3 & 4.12 & 13 & 0 \\
$\mathrm{O}_{2}$ & 125 & 3.86 & 13.2 & 0 \\
$\mathrm{O}$ & 700 & 2.4 & 11 & 0 \\
$\mathrm{O}_{3}$ & 211 & 4.82 & 13 & 0 \\
$\mathrm{NO}$ & 112.9 & 3.97 & 12.2 & 0 \\
$\mathrm{NO}_{2}$ & 348.4 & 4.27 & 13 & 0 \\
$\mathrm{~N}_{2} \mathrm{O}$ & 250 & 4.257 & 13 & 0 \\
$\mathrm{H}_{2}$ & 36.4 & 3.43 & 11.1 & 0 \\
$\mathrm{NH}_{3}$ & 474 & 3.44 & 13 & 441 \\
$\mathrm{CH}_{4}$ & 154.1 & 4.22 & 13 & 0
\end{tabular}

Table 10-3. Unlike-pair Lorenz-Berthelot coefficients. a

\begin{tabular}{lccc} 
System & $\mathrm{k}_{\mathrm{ij}}$ & $\mathrm{l}_{\mathrm{ij}}$ & $\mathrm{m}_{\mathrm{ij}}$ \\
\hline $\mathrm{N}_{2}-\mathrm{N}$ & 1 & 1 & 0.9 \\
$\mathrm{NO}-\mathrm{N}$ & 1 & 1 & 0.93 \\
$\mathrm{O}_{2}-\mathrm{O}$ & 1 & 1 & 0.98 \\
$\mathrm{~N}_{2}-\mathrm{NO}$ & 1 & 1 & 1 \\
$\mathrm{~N}_{2},-\mathrm{O}_{2}$ & 1 & 1 & 1.04 \\
$\mathrm{O}_{2}-\mathrm{NO}$ & 1 & 1 & 0.93 \\
$\mathrm{CO}_{2}, \mathrm{O}$ & 1 & 0.905 & 1 \\
$\mathrm{~N}_{2}-\mathrm{H}_{2} \mathrm{O}$ & 1 & 1.045 & 1 \\
$\mathrm{~N}_{2}-\mathrm{CO}_{2}$ & 1 & 1.035 & 1 \\
$\mathrm{CO}_{2}-\mathrm{H}_{2} \mathrm{O}$ & 1 & 0.9765 & 0.9 \\
$\mathrm{CO}_{2}-\mathrm{H}_{2} \mathrm{O}$ & 1 & 0.983 & 0.9 \\
$\mathrm{NH}_{3}-\mathrm{H}_{2} \mathrm{O}$ & 1 & $0.972 ?$ & $0.95 ?$
\end{tabular}


Table 10-4. Calculated changes with time of chemical species (in mol/mol) after detonation of $F$ in a calorimetry test. The initial time $(t=0)$ corresponds to the time when the expanding gases stopped by the wall of the calorimetry bomb and the resulting shock waves heat the gas to an in temperature of $2078 \mathrm{~K}$. Experimental data are for a heavily confined calorimatry sample is give "Eqm" indicates chemical equilibrium at all temperatures.

\begin{tabular}{ccccccccc}
$\begin{array}{c}\text { Time } \\
(\mathrm{s})\end{array}$ & $\begin{array}{c}\text { Temp. } \\
(\mathrm{K})\end{array}$ & graphite & $\mathrm{CO}$ & $\mathrm{CO}_{2}$ & $\mathrm{CH}_{4}$ & $\mathrm{H}_{2}$ & $\mathrm{H}_{2} \mathrm{O}$ & $\mathrm{N}_{2}$ \\
\hline 0 & 2078 & 0 & 1.63 & 3.38 & $4.1 \mathrm{E}-09$ & 0.37 & 3.64 & 2.0 \\
0.2 & 2035 & 0 & 1.61 & 3.40 & $3.6 \mathrm{E}-09$ & 0.39 & 3.62 & 2.0 \\
1.2 & 1832 & 0 & 1.56 & 3.46 & $1.8 \mathrm{E}-08$ & 0.45 & 3.57 & 2.0 \\
4.1 & 1351 & 0 & 1.36 & 3.65 & $5.4 \mathrm{E}-07$ & 0.65 & 3.37 & 2.0 \\
5.4 & 1186 & 0 & 1.34 & 3.68 & $5.8 \mathrm{E}-07$ & 0.67 & 3.34 & 2.0 \\
10.9 & 684 & 0 & 1.33 & 3.68 & $5.8 \mathrm{E}-07$ & 0.67 & 3.34 & 2.0 \\
22.0 & 349 & 0 & 1.33 & 3.68 & $5.8 \mathrm{E}-07$ & 0.67 & 3.34 & 2.0 \\
39.3 & 300 & 0 & 1.33 & 3.68 & $5.8 \mathrm{E}-07$ & 0.67 & 3.34 & 2.0 \\
100.0 & 298 & 0 & 1.33 & 3.68 & $5.8 \mathrm{E}-07$ & 0.67 & 3.34 & 2.0 \\
\hline Eqm & 298 & 0.94 & $1.0 \mathrm{E}-10$ & 4.03 & 0.03 & $9.8 \mathrm{E}-06$ & 3.94 & 2 \\
expt & 298 & 0 & $1.61(1)$ & $3.33(7)$ & 0 & $0.34(1)$ & $3.68(2)$ & $1.95(2)$
\end{tabular}

a. Numbers within parentheses denote experimental uncertainties of the last digits in data, e.g., $1.61(1)=1.61 \pm 0.01$.

Table 10-5. CHEQ calculations for $\omega$ and $E_{0}(\infty)$ with the $1800 \mathrm{~K}$ freeze and equlibrium (Eqm.) at all temperatures. The difference is significant. The numbers in parentheses are densities in $\mathrm{g} / \mathrm{cc}$.

\begin{tabular}{ccc|cc} 
& \multicolumn{2}{c|}{$\omega$} & \multicolumn{2}{c}{$\mathrm{E}_{\mathrm{o}}(\infty)(\mathrm{kJ} / \mathrm{cc})$} \\
\cline { 2 - 5 } Explosive & Freeze & Eqm. & Freeze & Eqm. \\
\hline TNM & 0.37 & 0.37 & 3.7 & 3.7 \\
RDX & 0.35 & 0.20 & 10.2 & 11.9 \\
RX-39-AB & 0.34 & 0.20 & 11.8 & 13.4 \\
HMX (1.89) & 0.34 & 0.24 & 11.4 & 12.8 \\
BTF & 0.33 & 0.12 & 11.2 & 14.5 \\
LX-14 & 0.33 & 0.22 & 10.6 & 12.0 \\
PETN $(1.76)$ & 0.29 & 0.18 & 11.2 & 12.6 \\
HNS $(1.66)$ & 0.27 & 0.11 & 8.1 & 11.0 \\
LX-17 & 0.26 & 0.19 & 8.6 & 9.4 \\
TATB & 0.25 & 0.18 & 8.4 & 9.4 \\
HNB & 0.21 & 0.21 & 15.2 & 15.2
\end{tabular}




\section{Appendix A: Explosives and their Properties}

The explosives used for detailed JWL analysis in this report are described in the next two tables. Table A-1 lists the mixture names, many particular to LLNL, and their compositions. The explosives and binders in these compositions and then listed with short names. In table A-2, the pure explosive and binder compounds are described: chemical composition, density (hopefully, the theoretical material density -TMD), molecular weight, and the heat of formation. Included are pure components that are seen only in solutions, eg. the RX-23 series, FM-1 and NNE. Also included are impurities like water and methanol that get into and degrade many of the liquid solutions.

Table A-3 lists pure explosive calorimetric total heats of detonation. These are converted linearly into cylinder density heats and hence into energies. This requires a small correction for PV and a larger one to convert liquid water to the gaseous form. CL-20 and TNAZ are recent measurements. ${ }^{1}$ The cylinder energies are linearly transformed into the expected energies at the theoretical maximum density (TMD), which allows direct comparison. A linear change of $\mathrm{E}_{0}$ seems legimate over a narrow range of densities, even though only high oxygen explosives actually follow this rule (see Chapter 2).

\section{References}

1. Measured by Don Ornellas, LLNL (unpublished). 
Table A-1. List of explosive and binder compositions.

\begin{tabular}{|c|c|c|c|}
\hline Explosive & Composition of Mixtures (wt\%) & Explosive & Composition of Mixtures (wt\%) \\
\hline AFX-902 & NQ 95, Viton-A 5 & $\mathrm{RX}-45-\mathrm{AA}$ & ANTA 95, Kel-F 5 \\
\hline Amatol & TNT 80-40, AN 20-60. Also Amatex & RX-47-AA & CL-14 92.514, Kel-F 7.486 \\
\hline ANFO & AN 94, diesel fuel oil 6 & RX-48-AA & ADNBF 92.37, Kel-F 7.63 \\
\hline ANFO-1.248 & AN 77, H2O 16, mineral oil 6, emulsifier 1 & $\mathrm{RX}-52-\mathrm{AD}$ & TATB 65, FEFO 31, pcl 1.6, pvf 1.4, n-100 1.1 \\
\hline Baratol & Ba nitrate 76 , TNT 24 & $\mathrm{RX}-52-\mathrm{AE}$ & TATB 65, FEFO, 32, pcl, 1.6, pvf, 1.4 \\
\hline $\mathrm{C}-4$ & RDX 91, DOS, 5.3, PIB 2.1, motor oil 1.6 & RX-HD & AN 79, Ca nitrate 6 , water 9 , fuel oil 6 \\
\hline Comp A & $\mathrm{RDX} 91$, wax 9 & $\mathrm{~T} 2$ & TATB 97, unknown binder 3 \\
\hline Comp B & RDX 63, TNT 36, Wax 1 & $\mathrm{X}-0219$ & TATB 90, kel-F 10 \\
\hline Cyclotol & RDX 75, TNT 25 & $\mathrm{X}-0290$ & see $\mathrm{PBX}-9502$ \\
\hline FM-1 & FEFO 23, MF-1 52, BDNPF 25 & $\mathrm{X}-0309$ & TNT $75, \mathrm{Al} 19$, wax 5 , acetylene black 2 \\
\hline HANFO & AN 72.4, FO 4.6, AN/water/emulsion 23 & $\mathrm{x}-0341$ & TATB 90, HMX 5, Kel-F 5 \\
\hline IRX-1 & HMX 53, НTPB 47 & $\mathrm{X}-0342$ & TATB 85, HMX 10, Kel-F 5 \\
\hline IRX-3A & HMX 58, Al 6, HTPB 36 & $\mathrm{X}-0343$ & TATB 81, HMX 14, Kel-F 5 \\
\hline $\operatorname{IRX}-4$ & HMX 30, AP 24, Al 16, HTPB 30 & $\mathrm{X}-0344$ & TATB 71, HMX 24, Kel-F 5 \\
\hline LX-04 & HMX 85, Viton-A 15 & XTX-8003 & PETN 80 , silicone rubber 20 \\
\hline $\mathrm{LX}-10$ & HMX 95, Viton-A 5 & & \\
\hline $\mathrm{LX}-14$ & HMX 95.5, estane 4.5 & Abbrev & Short Explosive Name \\
\hline $\mathrm{LX}-17$ & TATB 92.5, kel-F 7.5 & ADNBF & amino-dinitro-benz-oxadiazole \\
\hline LXं-19 & see RX-39-AB. Also was RX-39-AC & AN & ammonium nitrate \\
\hline NNE & NM 39, NP 56, ED 5 & ANTA & amino-nitro-triazole \\
\hline Octol & HMX 75, TNT 25 & AP & ammonium perchlorate \\
\hline PBX-9011 & HMX 90, estane 10 & BTF & benzotri-furoxan \\
\hline PBX-9404 & HMX 94, NC 3, CEF 3 & CL-14 & diamino-dinitro-benzofuroxan \\
\hline PBX-9407 & RDX 94, Exon(461) 6 & CL-20 & $\varepsilon$-heaxnitroazaiso-wurzitane \\
\hline PBX-9501 & HMX 95, estane 2.5, BDNPA-F 2.5 & 1,2-DP & bis-difluoroamino-propane \\
\hline PBX-9502 & TATB 95, Kel-F 5 & DNT & dinitro-toluene \\
\hline PBXN-110 & HMX 88, HTPB 12 & ED & ethylene diamine \\
\hline PBNX-111 & RDX 20, AP 43, Al 25, HTPB 12 & FEFO & bis-fiuoro-dinitroethyl-formal \\
\hline PBXW-115 & see $\mathrm{PBXN}-111$ & FO & fuel oil \\
\hline PBXW-123 & AP $45, \operatorname{Al} 30$, TMETN 19, other 6 & HMX & octahydro-tetranitro-tetraocine \\
\hline Pentolite & PETN 50, TNT 50 & $\mathrm{HNX}$ & high nitrogen explosive (LANL) \\
\hline QM-100 & AN 75 , water 18 , fuel oil 5 , plastic balloons 2 & $\mathrm{HNNB}$ & hexanitro-benzene \\
\hline $\mathrm{RX}-23-\mathrm{AA}$ & hydrazine nitrate 79 , hydrazine 21 & HNS & hexanitro-stilbene \\
\hline $\mathrm{RX}-23-\mathrm{AB}$ & hyd nitrate 69 , hydrazine 5 , water 26 & $\mathrm{~K}-6$ & oxo-trinitro-triazocyclohexane \\
\hline $\mathrm{RX}-23-\mathrm{AC}$ & hyd nitrate 32 , hydrazine 68 & MF-1 & fluoro-dinitoethyl-propyl-formal \\
\hline $\mathrm{RX}-26-\mathrm{AF}$ & HMX 49.3, TATB 46.6 , estane 4.1 & NM & nitro-methane \\
\hline $\mathrm{RX}-27-\mathrm{AD}$ & TACOT 92.5, kel-F 7.5 & NP & nitro-propane \\
\hline $\mathrm{RX}-36-\mathrm{AH}$ & HMX 51.3, BTF 43.7, Viton-A 5.0 & $\mathrm{NQ}$ & nitro-guanidine \\
\hline $\mathrm{RX}-39-\mathrm{AB}$ & CL-20(epsilon) 95.8, estane 4.2 & PETN & pentaerythritol tetranitrate \\
\hline $\mathrm{RX}-41-\mathrm{AB}$ & K-6 95, Viton-A 5 & $\mathrm{PF}$ & picryl-fluoride \\
\hline $\mathrm{RX}-43-\mathrm{AC}$ & HNX, 95, DER, 5 & RDX & hexahydro-trinitro-triazine \\
\hline
\end{tabular}


Table A-1, part 2

\begin{tabular}{ll|ll} 
Explosive & Composition of Mixtures $($ wt $\%)$ & Abbrev & Short Binder/Filler Name \\
\hline TACOT & tetranitro-benzotriazol-hydroxide & DOS & di(2-ethylhexyl)stearate \\
TATB & triamino-trinitro-benzene & estane & polyrurethane solution system \\
Tetryl & methyl, tetranitrobenzamine & HTBP & hydroxy-terminated polybutadiene \\
TNAZ & trinitro-azetidine & kel-F & chlorotrifluoroethylene/ vinylidine polymer \\
TNGU & tetranitro-gylcoluril & NC & nitrocellulose \\
TNT & trinitro-toluene & PCL & polycaprolactone \\
& & PIB & poly-isobutylene \\
Abbrev & Short Binder/Filler Name & PMMA & poly-methyl-methacrylate \\
BDNPA-F & mix of bis-dinitropropyl acetal/formal & PVF & poly-vinyl-formal \\
CEF & tris-b-chloroethyl phosphate & Viton-A & vinylidine fluoride/hexa-fluoropropylene copolymer \\
DER & DER-332 (epoxy) & &
\end{tabular}

Table A-2. Detailed data on individual compounds. $\mathrm{S}$ is a solid; $\mathrm{L}$ a liquid.

\begin{tabular}{|c|c|c|c|c|c|c|c|c|c|c|c|}
\hline $\begin{array}{l}\text { Common } \\
\text { Name }\end{array}$ & Formula & & $\begin{array}{l}\rho_{0} \text { or } \\
\mathrm{TMD}^{*} \\
\text { (g/cc) }\end{array}$ & $\begin{array}{l}\text { Molec- } \\
\text { ular } \\
\text { Weight } \\
\text { (g/mol) }\end{array}$ & $\begin{array}{c}\text { Heat of } \\
\text { Forma- } \\
\text { tion } \\
(\mathrm{kJ} / \mathrm{mol})\end{array}$ & $\begin{array}{l}\text { Common } \\
\text { Name }\end{array}$ & Formula & & $\begin{array}{l}\rho_{0} \text { or } \\
\mathrm{TMD}^{*} \\
\text { (g/cc) }\end{array}$ & $\begin{array}{l}\text { Molec- } \\
\text { ular } \\
\text { Weight } \\
\text { (g/mol) }\end{array}$ & $\begin{array}{l}\text { Heat of } \\
\text { Forma- } \\
\text { tion } \\
(\mathrm{kJJ} / \mathrm{mol})\end{array}$ \\
\hline$\overline{\mathrm{ADNBF}}$ & C6H3N5O6 & $\overline{\mathbf{S}}$ & 1.91 & 241.12 & 153.9 & $\mathrm{NM}$ & CH3NO2 & $\bar{L}$ & $1.13^{*}$ & 61.04 & -113.1 \\
\hline $\mathrm{AN}$ & $\mathrm{H} 4 \mathrm{~N} 2 \mathrm{O} 3$ & $\mathbf{S}$ & 1.72 & 80.04 & -365.1 & NP & C3H7NO2 & $\mathrm{L}$ & 0.988 & 89.09 & -180.3 \\
\hline BTF & C6N606 & $S$ & $1.901^{*}$ & 252.11 & 601.7 & PF & $\mathrm{C} 6 \mathrm{H} 2 \mathrm{~N} 3 \mathrm{O} 6 \mathrm{~F}$ & $\mathbf{L}$ & $1.833^{*}$ & 231.1 & -259.4 \\
\hline CL-14 & $\mathrm{C} 6 \mathrm{H} 4 \mathrm{~N} 6 \mathrm{O} 6$ & $\mathbf{S}$ & $1.942 *$ & 256.13 & 86.2 & RDX & C3H6N6O6 & $\mathbf{S}$ & $1.806^{*}$ & 222.1 & 61.6 \\
\hline CL-20 & C6H6N12O12 & $\mathbf{S}$ & $2.044^{*}$ & 438.19 & 377.2 & TACOT & C12H4N8O8 & $\mathbf{S}$ & $1.85^{*}$ & 388.2 & 462.3 \\
\hline DNB & $\mathrm{C} 6 \mathrm{H} 4 \mathrm{~N} 2 \mathrm{O} 4$ & $\mathbf{S}$ & $1.575^{*}$ & 168.11 & -25.94 & TATB & $\mathrm{C} 6 \mathrm{H} 6 \mathrm{~N} 6 \mathrm{O} 6$ & $\mathbf{S}$ & $1.938^{*}$ & 258.16 & -154.2 \\
\hline HN & C0.1H4.9N2.7O2.8 & $\mathbf{L}$ & 1.665 & 88.32 & -250.2 & CEF & C6H12Cl3O4P & $\mathbf{L}$ & 1.425 & 285.5 & -1255 \\
\hline HNB & C6N6012 & $\mathbf{S}$ & $2.02 *$ & 348.11 & 65.69 & ED & $\mathrm{C} 2 \mathrm{H} 8 \mathrm{~N} 2$ & $\mathbf{L}$ & 0.900 & 60.11 & -63.01 \\
\hline HNO3 & HNO3 & $\mathbf{L}$ & 1.503 & 63.01 & -173.2 & estane & C5.1H7.5N0.2O1.8 & $\mathbf{S}$ & 1.16 & 194.7 & -773 \\
\hline HNS & C14H6N6O12 & $\mathbf{S}$ & $1.74^{*}$ & 450.3 & 78.24 & $\mathrm{H} 2 \mathrm{O}$ & $\mathrm{H} 20$ & $\mathbf{L}$ & 1.00 & 18.015 & -285.8 \\
\hline $\mathrm{HNX}$ & C8H5N13O6 & $\mathbf{S}$ & $1.84^{*}$ & 379.21 & 437.2 & Kel-F & C8H2F11Cl3 & $\mathbf{S}$ & 2.02 & 413.5 & -2418 \\
\hline HYD & $\mathrm{H} 4 \mathrm{~N} 2$ & $\mathbf{L}$ & 1.011 & 31.88 & 46.23 & $\mathrm{NC}$ & C6.0H7.3N2.7O10.4 & $\mathbf{S}$ & 1.656 & 283.9 & -682 \\
\hline $\mathrm{K}-6$ & C3H4N6O7 & $\mathbf{S}$ & 1.932 & 236.1 & -41.84 & Viton A & $(\mathrm{C} 5 \mathrm{H} 3.5 \mathrm{~F} 6.5) \mathrm{n}$ & $\mathbf{S}$ & 1.85 & $(187.8) \mathrm{n}$ & -1392 \\
\hline MF-1 & C6H9N4O10F & $\mathbf{L}$ & 1.534 & 316.16 & -669.9 & & & & & & \\
\hline
\end{tabular}


Table A-3. List of calorimetrically-derived energies of detonation.

\begin{tabular}{|c|c|c|c|c|c|c|c|}
\hline \multirow[b]{2}{*}{ Explosive } & \multirow{2}{*}{$\begin{array}{c}\text { Theoret. } \\
\text { Material } \\
\text { Density } \\
\text { TMD } \\
\text { (g/cc) }\end{array}$} & \multirow{2}{*}{$\begin{array}{c}\text { Calori- } \\
\text { metric } \\
\text { Density } \\
(\mathrm{g} / \mathrm{cc})\end{array}$} & \multirow{2}{*}{$\begin{array}{c}\text { Measured } \\
\text { Confined } \\
\text { Heat of } \\
\text { Detonation } \\
(\mathrm{kJ} / \mathrm{cc})\end{array}$} & \multirow{2}{*}{$\begin{array}{c}\text { Cyl- } \\
\text { inder } \\
\text { Densities } \\
(g / c c)\end{array}$} & \multirow{2}{*}{$\begin{array}{c}\text { Adjusted } \\
\text { Cylinder } \\
\text { Heat of } \\
\text { Detonation } \\
(\mathrm{kJ} / \mathrm{cc})\end{array}$} & \multicolumn{2}{|c|}{$\begin{array}{c}\text { Energy of } \\
\text { Detonation } \\
(\mathrm{kJ} / \mathrm{cc})\end{array}$} \\
\hline & & & & & & $\begin{array}{l}\text { Cyl- } \\
\text { inder }\end{array}$ & $\begin{array}{c}\text { at } \\
\text { TMD }\end{array}$ \\
\hline HNB & 2.02 & 1.918 & 13.27 & 1.965 & 13.6 & 13.7 & 14.4 \\
\hline e-CL-20 & 2.044 & 1.956 & 12.19 & 1.942 & 12.1 & 11.8 & 12.3 \\
\hline BTF & 1.901 & 1.86 & 10.98 & 1.852 & 10.9 & 11.2 & 11.4 \\
\hline $\mathrm{HMX}$ & 1.905 & 1.89 & 11.70 & 1.893 & 11.7 & 11.0 & 11.1 \\
\hline TNAZ & 1.83 & 1.83 & 11.21 & 1.83 & 11.2 & 11.0 & 11.0 \\
\hline PETN & 1.78 & 1.732 & 10.81 & 1.763 & 11.0 & 10.3 & 10.6 \\
\hline PETN & 1.78 & 1.496 & 9.41 & 1.503 & 9.5 & 8.9 & \\
\hline FEFO & 1.607 & 1.6 & 8.56 & 1.607 & 8.6 & 8.3 & 8.3 \\
\hline HNS & 1.74 & 1.649 & 7.58 & 1.655 & 7.6 & 7.5 & 7.9 \\
\hline TATB & 1.938 & 1.87 & 7.96 & 1.86 & 7.9 & 7.5 & 7.7 \\
\hline $1,2-\mathrm{DP}$ & 1.26 & 1.265 & 7.09 & 1.26 & 7.1 & 7.2 & 7.2 \\
\hline TNT & 1.654 & 1.533 & 7.01 & 1.632 & 7.5 & 7.1 & 7.7 \\
\hline $\mathrm{RX}-23-\mathrm{AA}$ & 1.424 & 1.424 & 7.98 & 1.424 & 8.0 & 6.5 & 6.5 \\
\hline HMX & 1.905 & 1.20 & 6.63 & 1.188 & 6.6 & 6.2 & \\
\hline $\mathrm{NM}$ & 1.13 & 1.13 & 5.80 & 1.13 & 5.8 & 5.2 & 5.2 \\
\hline$R X-23-A B$ & 1.356 & 1.356 & 6.24 & 1.356 & 6.2 & 4.3 & 4.3 \\
\hline RX-23-AC & 1.136 & 1.136 & 4.66 & 1.136 & 4.7 & 4.1 & 4.1 \\
\hline HNS & 1.74 & 1.017 & 3.74 & 1.001 & 3.7 & 3.7 & \\
\hline
\end{tabular}




\section{Appendix B. Impedance Calculations}

\section{Simple Flyer into Explosive}

The original calculation to determine the pressure created by a mylar flyer of initial velocity $\mathrm{u}$ at the instant of detonation is as follows. 1,2 The density, resulting wave velocity and resulting particle velocity of flyer and explosive are $\rho_{f}, U_{f}$, and $u_{f}, \rho_{o}, U_{S}$ and $u_{p}$. The assumption is made that the pressures of the flyer and the explosive are equal, ie. that

$$
P_{f}=P_{e}
$$

This is true only if the mylar and unreacted explosive have identical impedances. At impact, we have the impedance relations

$$
\rho_{f} U_{f} u_{f}=\rho_{o} U_{s} u_{p}
$$

where

$$
u_{f}=u-u_{p}
$$

The Hugoniot of the mylar (density $1.41 \mathrm{~g} / \mathrm{cc}$ ) is $U_{f}=A+B u_{f}$, where $A=2.27 \mathrm{~mm} / \mu \mathrm{s}$ and $B$ $=1.56 .{ }^{3}$ The Hugoniot of the unreacted explosive is $U_{S}=C_{0}+S_{1} u_{p}$. We substitute and eliminate uf

$$
\rho_{f}\left[A+B\left(u-u_{p}\right)\right]\left(u-u_{p}\right)=\rho_{o}\left(C_{o}+S_{1} u_{p}\right) u_{p}
$$

This becomes

$$
\left(\rho_{f} B-\rho_{o} S_{1}\right) u_{p}^{2}-\left[\rho_{f}(A+2 B u)+\rho_{o} C_{o}\right] u_{p}+\rho_{f} u(A+B u)=0
$$

The negative root of the quadratic equation is the solution for $\mathbf{u}_{\mathbf{p}}$. From this, everything else may be calculated using Hugoniots and impedance relations. 


\section{Simple Forward Detonation into a Plate}

We digress to a second classic impedance problem because we need one of its assumptions to continue work on the first problem. This problem is an explosive hitting a metal plate head-on. Because the impedance of the metal is higher than that of the explosive, we expect a high pressure in the metal and a reflected shock wave. The pressures at the instant of collision are:

$$
P_{m}=P_{e}+P_{r}
$$

where the terms refer to metal, the explosive's spike state and the reflected wave.

The next assumption is to set the reflected pressure proportional to 4,5

$$
P_{r} \sim \rho_{r} U_{r}\left(u_{p}-u_{m}\right)
$$

where $\rho_{r}$ and $U_{r}$ are the density and wave velocity in the reflected wave and $u_{m}$ is the velocity of the metal plate where struck. The Acoustic Approximation is made at this point, ie

$$
\rho_{\mathrm{r}} \mathrm{U}_{\mathrm{r}}(\text { reacted }) \sim\left(4 / 3 \rho_{0}\right)(3 / 4 \mathrm{D}) \sim \rho_{\mathrm{O}} \mathrm{D} .
$$

Using the momentum equations $P_{m}=\rho_{m} U_{m} u_{m}$ and $u_{p}=P d / \rho_{0} U_{S}$ we assemble all the above into

$$
P_{e}=\left(\rho_{o} D+\rho_{m} U_{m}\right) \frac{u_{m}}{2}
$$

Using $u_{m}=u_{S} / 2$, the measured free-face jump-off velocity, the calculated value of $P_{e}$ constituted the first measurement of the detonation pressure of an explosive. The above process has the big advantage that $u_{p}$ never need be known.

Originally, Eq. 9 was used with the assumption that all the explosive is reacted, even though we never had to select a Hugoniot. The assumption that $F=1$ made here contrasts with the usual $F$ $=0$ assumption made in section 1 for the reverse case with the flyer. Applying Eq. 9 gives the right detonation pressure for LX-14 but a high result of $34 \mathrm{GPa}$ for $\mathrm{LX}-17$. This is the result of seeing the spike so that $\mathbf{F}$ cannot really be 1 . 
Suppose that, at the instant of impact, that $F=0$. Then $\rho_{r} U_{r}=\rho_{0} D$ again, because the front of the reaction is moving at the detonation velocity, $\mathrm{D}$. Also, $\mathrm{u}_{\mathrm{p}}=\mathrm{P}_{\mathrm{e}} / \rho_{\mathrm{o}} \mathrm{D}$ at the detonation point and $u_{k}=P_{k} / \rho_{o} D$ at the spike point. Thus, in an explosive at steady state, $u_{k}>u_{p}$. We then get the same result, except we used unburned variables. The calculation, being centered on $u_{m}$ cannot tell whether any explosive is burned or not.

Eqs. 7 and 9 are valid also for the case of the explosive hitting a plate of lower impedance. In this case, $u_{m}>u_{f}$ and $P_{r}$ will be negative. This is the rarifaction that results when the impedance goes from higher to lower.

\section{Improved Flyer to Explosive Problem}

We now take part of section 2 and feed it back into the problem of section 1 . The impedances (density times wave velocity, $\rho U$ ) of the flyer and explosive are never the same. We have not considered the effect of the reflected pressure or rarifaction waves. For mylar moving into an explosive, the pressure generated in the explosive will be higher than in the flyer and a reflected pressure wave, $P_{\mathbf{r}}$, will move backward in the flyer from the interface. For a metal hitting an explosive, a rarifaction (with negative pressure) will be relflected back. ${ }^{6}$ At the instant of impact, the pressures will be:

$$
P_{f}+P_{r}=P_{e}
$$

From Deal's treatment of the pressure of an explosive hitting a metal plate head-on, ${ }^{5}$ we describe the reflected wave in terms of the particle velocities we know.

$$
P_{r}=\rho_{f} U_{f} u_{r}=\rho_{f} U_{f}\left(u_{f}-u_{p}\right)
$$

where $u_{f}>u_{p}$ so that

$$
\rho_{f} U_{f}\left(u-u_{p}\right)+\rho_{f} U_{f}\left(u_{f}-u_{p}\right)=\rho_{o} U_{s} u_{p}
$$

Because $u_{f}=u-u_{p}$, we have

$$
u_{f}-u_{p}=u-2 u_{p}
$$

The explosive Hugoniot becomes 


$$
U_{s}=(1-F)\left(C_{0}+S_{1} u_{p}\right)+F\left(M+N u_{p}\right)
$$

where $F$ is the burn fraction and $M$ and $N$ are the coefficients of the reacted explosive. For $L X-17$, the reacted Hugoniot is $U_{\mathrm{S}}=5.85+0.83 \mathrm{u}_{\mathrm{p}}$ for $2.2<\mathrm{u}_{\mathrm{p}}<3.2 \mathrm{~mm} / \mu \mathrm{s}$ as obtained from

supracompressed gun experiments. 7 This approach ignores the creation of a detonation pressure and treats the reacted products as being inert.

Substituting, we have the quadratic equation

$$
\begin{aligned}
\rho_{f}\left\{\left[A+B\left(u-u_{p}\right)\right]\left(u-u_{p}\right)+\right. & {\left.\left[A+B\left(u-2 u_{p}\right)\right]\left(u-2 u_{p}\right)\right\}=} \\
& \rho_{o}\left[(1-F)\left(C_{o}+S_{1} u_{p}\right)+F\left(M+N u_{p}\right)\right] u_{p}
\end{aligned}
$$

which we rearrange into the form

$$
a u_{p}^{2}+b u_{p}+c=0
$$

where

$$
\begin{aligned}
& a=5 \rho_{f} B-\rho_{o}\left[(1-F) S_{1}+F N\right] \\
& b=-\left\{3 \rho_{f}(A+2 B u)+\rho_{o}\left[(1-F) C_{o}+F M\right]\right\} \\
& c=2 \rho_{f} u(A+B u)
\end{aligned}
$$

Again, the negative root is the solution.

Some comparative results shown in Table B-1 for mylar, aluminum and steel on LX-17, illustrating the effect of having different impedances. A mylar flyer is closely matched in impedance to the explosive and there is little difference between the unreacted and reacted calculations. If these calculations are done correctly, the resulting $\mathrm{P}-\mathrm{v}$ equation for the $\mathrm{F}=0$ explosive should be the one obtained from the $U_{S}-u_{p}$ Hugoniot. This is 8

$$
P_{e}=\rho_{0} C_{o}^{2} \frac{1-v}{\left[S_{1}(v-1)+1\right]^{2}}
$$


where

$$
v=1-u_{p} / U_{s}
$$

The solutions are listed in Table B-1. The impedances diminish in the order of steel $>$ aluminum $>$ reacted explosive $>$ unreacted explosive $>$ mylar. The steel solution works only $u_{p}$ to $u=3.8 \mathrm{~mm} / \mu \mathrm{s}$, then $b^{2}$ - 4 ac becomes negative. This represents the break-down of the too simplistic two-term $U_{\mathrm{S}^{-}}$ $u_{p}$ EOS when the impedances become very different.

Table B-2 is the kind of result generated for general Electric Gun use. It is for LX-14 over a broad range of 10-mil Mylar flyer velocities. An unreacted Hugoniot of $U_{S}=2.9+2.06 u_{p}$ is used. 9 Use of a reacted Hugoniot lowers the pressures by only about 1 or $2 \mathrm{GPa}$. The pulse time, $\tau_{\mathrm{f}}$, is taken as the time for the waye velocity to travel from back and forth across the compressed flyer. For $x_{f}$ and $v_{f}$, the width and compressed relative volume of the flyer, the pulse time is

$$
\tau_{f} \approx \frac{2 v_{f} x_{f}}{U_{f}}=\frac{2 x_{f}}{U_{f}}\left(1-\frac{u_{f}}{U_{f}}\right) .
$$

The resulting explosive pressure, $\mathrm{P}_{\mathrm{e}}$, and the pulse time, $\tau_{\mathrm{f}}$, may be used as direct input into a hydrocode. The initial flyer velocity relates it to a real experiment. The $u_{f}$ and $U_{f}$ quantities come from the impedance calculations done above.

Finally, we compare the impedance results with those from program burn/beta burn and Ignition \& Growth (I\&G). All are in agreement at high pressures. The impedance calculations are lower from the detonation pressure region on down. Below the detonation pressure, the spikes created in the two codes are different. I\&G appears to be generous in putting in pressure at low flyer velocities. The real problem is trying to be sure of what the first real pressure is in an explosive. As we see, the results can be uncertain. 


\begin{tabular}{ccccccc} 
& \multicolumn{6}{c}{ Impact Pressures, $\mathrm{P}_{\mathrm{e}}$} \\
\cline { 2 - 7 } $\mathrm{u}$ & unreact & react & $\begin{array}{c}\text { Impedance } \\
\text { spike }\end{array}$ & $\begin{array}{c}\text { PB/BB } \\
\text { plateau }\end{array}$ & $\begin{array}{c}\text { I\&G } \\
\text { spike }\end{array}$ & $\begin{array}{c}\text { I\&G } \\
\text { plateau }\end{array}$ \\
\hline 2.3 & 16 & 21 & 26 & 25 & 30 & 25 \\
3.0 & 27 & 30 & 34 & 33 & 34 & 33 \\
3.8 & 44 & 45 & 45 & 43 & 44 & 44
\end{tabular}

\section{Defining the Instant of Impact}

All impedance calculations hold for the instant of impact, but how long is that? Fig. B-1 shows data from two cylinder and two electric gun thin-metal plate shots. The cylinder samples are PETN (\#511) and LX-17(\#523). The plates are PETN (ppe88003) and LX-17 (pta90002). The Fabry data was differentiated to get the acceleration. Finding good plate shots for this was difficult and smoothing was required before differentiation. The figure shows the jump-off in the rare cases where data was available. The second jump was also observed and noticed to be comparable and a little wider.

The total linewidths in Fig. B-1 are about $0.05 \mu$ s (about $0.25 \mathrm{~mm}$ in the metal) with the halfwidths at halfheight being about $0.02 \mu \mathrm{s}$. This holds whether the shot was straight-ahead or sideways and whether the explosive was ideal or not. The $\mathrm{LX}-17$ reaction zone width of about 0.25 $\mu \mathrm{s}$ is much larger than the width of the shock wave. It appears that the shock wave comes from a zone of constant thickness of about $1 / 4 \mathrm{~mm}$ in the explosive.

We would then expect the same explosive to deliver the same shock wave energy to a metal plate, ie. $E=\rho m^{u}{ }^{2} / 2$. Then, we expect

$$
u_{\mathrm{fs}} \approx \rho_{\mathrm{m}}{ }^{1 / 2}
$$

Fig. B-2 shows this plot for LX-14 and LX-17 without any concern for plate thickness. On the basis of this small sample, the relation is approximately true.

\section{References}

1. J. H. Kinecke, Jr. and C. E. West, Jr., "Shocked States of Four Overdriven Explosives", Proceedings Fifth Symposium (International) on Detonation, Pasadena, CA, August 18-21, 1970, p. 533. 
2. V. M. Boyle and D. L. Palarski, "Shock Initiation Sensitivity of Multiply-Shocked Pressed TNT," Proceedings Seventh Symposium (International) on Detonation, Annapolis, MD. JUne 16-19, 1981, p. 906.

3. F. Davies, Hugoniot Equation of State of Mylar, Boeing Co. report, No. 81205, D2-125304-1 (1969).

4. R. E. Duff and E. Houston, J. Chem. Phys. 231268 (1955).

5. W. E. Deal, J. Chem. Phys. 27796 (1957).

6. M. L. Wilkens, Introduction to Hydrodynamics, LLNL Report UCIR-660 (1972).

7. L. Green, E. Lee, A. Mitchell and C. Tarver, "The Supra-Compression of LX-07, LX-17, PBX-9404 and RX-26-AF and the equations of State of the Detonation Products," Proceedings Eighth Symposium (International) on Detonation, Albuquerque, NM, July 15-19, 1985, p. 587.

8. Previous report, p. 7-15.

9. B. M. Dobratz and P. C. Crawford, LLNL Explosives Handbook, LLNL Report UCRL-52997 revision 2 (1985), p. 7-32.

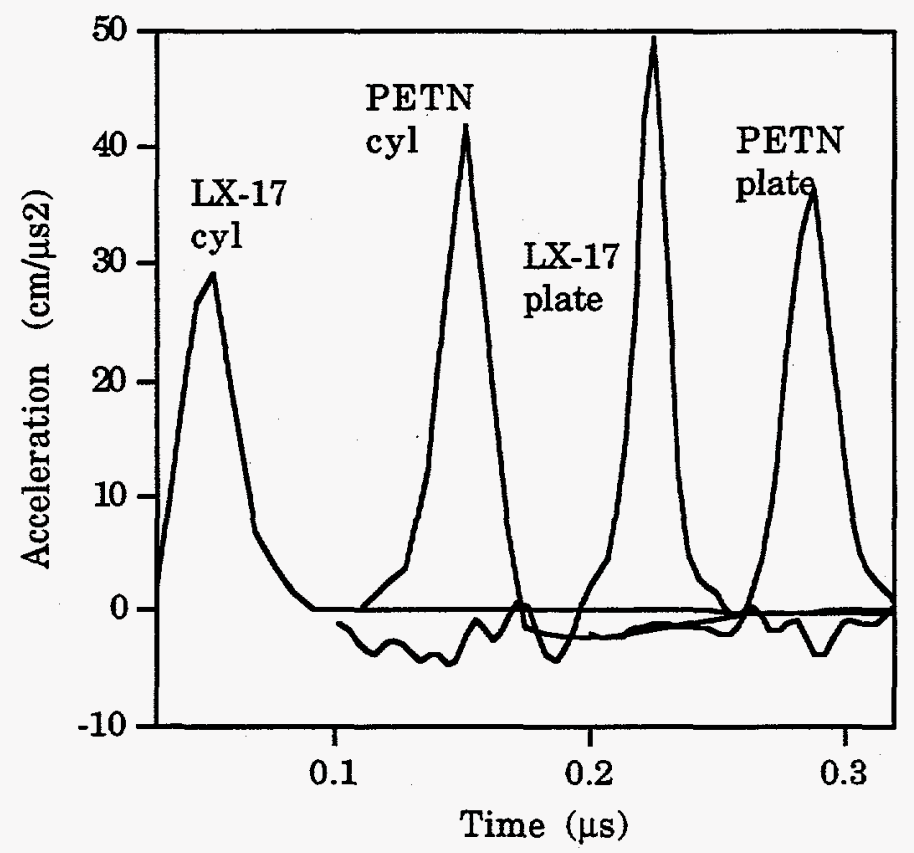

Fig. B-1. Shockwidths taken from LX-17 and PETN cylinder and plate shots. The Fabry data has been differentiated to acceleration. The halfwidths are about $0.02 \mu \mathrm{s}$. 


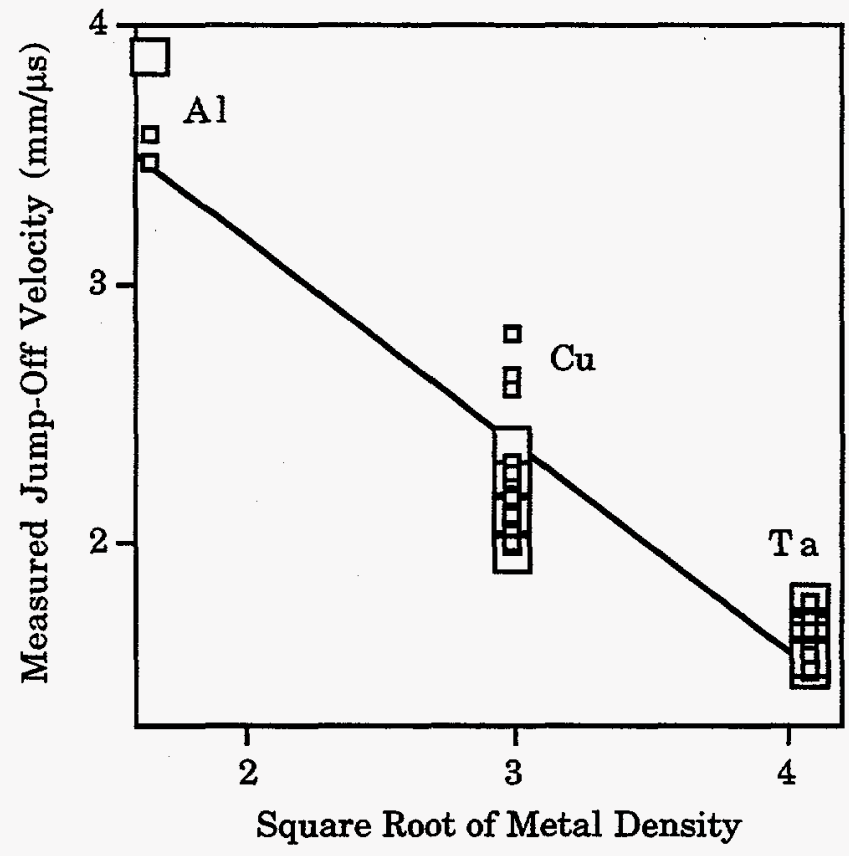

Fig. B-2. Plot of the measured unconfined face jump-off velocity versus the square root of the metal plate density for LX-14 (small squares) and LX-17 (large squares). The notion of a constant delivered shock wave energy is approximately true.

Table B-1. Calculated explosive properties using impedance formulas for three flyers on unreacted LX-17. All EOS's are two-term $U_{s}-u_{p}$.

\begin{tabular}{ccccccccc} 
& \multicolumn{2}{c}{ Velocity $(\mathrm{mm} / \mu \mathrm{s})$} & \multicolumn{3}{c}{ Velocity $(\mathrm{mm} / \mu \mathrm{s})$} & \multicolumn{3}{c}{ Pressure (GPa) } \\
\cline { 2 - 9 } Flyer & $\mathrm{u}$ & $\mathrm{u}_{\mathrm{f}}$ & $\mathrm{u}_{\mathrm{p}}$ & $\mathrm{U}_{\mathrm{f}}$ & $\mathrm{U}_{\mathrm{s}}$ & $\mathrm{P}_{\mathrm{f}}$ & $\mathrm{P}_{\mathrm{f}}$ & $\mathrm{P}_{\mathrm{e}}$ \\
\hline Mylar & 3.0 & 1.7 & 1.3 & 4.9 & 4.9 & 11 & 1 & 13 \\
& 4.0 & 2.2 & 1.8 & 5.7 & 5.8 & 18 & 2 & 20 \\
& 5.0 & 2.8 & 2.2 & 6.6 & 6.7 & 26 & 3 & 28 \\
& 6.0 & 3.3 & 2.7 & 7.5 & 7.6 & 35 & 3 & 39 \\
& 7.0 & 3.9 & 3.1 & 8.4 & 8.5 & 46 & 4 & 50 \\
& 8.0 & 4.5 & 3.5 & 9.2 & 9.4 & 58 & 5 & 63 \\
\hline Aluminum & 3.0 & 1.3 & 1.7 & 7.0 & 5.7 & 24 & -6 & 19 \\
& 4.0 & 1.7 & 2.3 & 7.7 & 6.8 & 36 & -7 & 29 \\
& 5.0 & 2.2 & 2.8 & 8.3 & 7.9 & 49 & -7 & 42 \\
& 6.0 & 2.7 & 3.3 & 8.9 & 9.0 & 65 & -8 & 57 \\
& 7.0 & 3.2 & 3.8 & 9.6 & 10.1 & 82 & -8 & 74 \\
& 8.0 & 3.6 & 4.4 & 10.2 & 11.1 & 101 & -8 & 92 \\
\hline Steel & 3.0 & 0.9 & 2.1 & 6.4 & 6.6 & 43 & -16 & 27 \\
& 3.5 & 0.9 & 2.6 & 6.5 & 7.5 & 47 & -11 & 36 \\
& 3.8 & 0.9 & 2.9 & 6.5 & 8.1 & 47 & -2 & 44
\end{tabular}


Table B-2. Calculated electric gun values for LX-14 using an unreacted Hugoniot. Results from a reacted Hugoniot are a couple of GPa lower. The explosive pressure, $P_{e}$, and the pulse time, $t_{f}$, can be used as input to a hydrocode. The initial velocity relates it to a 10-mil Mylar flyer.

\begin{tabular}{|c|c|c|c|c|c|c|c|}
\hline \multicolumn{4}{|c|}{10 mil Mylar Flyer } & \multicolumn{3}{|c|}{ Explosive } & \multirow{2}{*}{$\begin{array}{l}\text { Flyer } \\
\text { Pulse } \\
\text { Time } \\
t_{f}(\mu \mathrm{s})\end{array}$} \\
\hline $\begin{array}{c}\text { Initial } \\
\text { Velocity } \\
\mathbf{u}\end{array}$ & $\begin{array}{c}\text { Wave } \\
\text { Velocity } \\
U_{f}\end{array}$ & $\begin{array}{c}\text { Particle } \\
\text { Velocity } \\
u_{f}\end{array}$ & $\begin{array}{c}\text { Pres- } \\
\text { sure } \\
\mathrm{P}_{\mathrm{f}}\end{array}$ & $\begin{array}{c}\text { Wave } \\
\text { Velocity } \\
\mathrm{U}_{\mathrm{S}}\end{array}$ & $\begin{array}{c}\text { Particle } \\
\text { Velocity } \\
u_{p}\end{array}$ & $\begin{array}{c}\text { Pres- } \\
\text { sure } \\
\mathrm{P}_{\mathrm{e}}\end{array}$ & \\
\hline 3.0 & 4.9 & 1.7 & 12 & 5.6 & 1.30 & 13 & 0.068 \\
\hline 3.2 & 5.1 & 1.8 & 13 & 5.8 & 1.39 & 15 & 0.064 \\
\hline 3.4 & 5.3 & 1.9 & 14 & 5.9 & 1.48 & 16 & 0.061 \\
\hline 3.6 & 5.4 & 2.0 & 16 & 6.1 & 1.57 & 18 & 0.058 \\
\hline 3.8 & 5.6 & 2.1 & 17 & 6.3 & 1.65 & 19 & 0.056 \\
\hline 4.0 & 5.8 & 2.3 & 18 & 6.5 & 1.74 & 21 & 0.054 \\
\hline 4.2 & 6.0 & 2.4 & 20 & 6.7 & 1.83 & 22 & 0.051 \\
\hline 4.4 & 6.1 & 2.5 & 22 & 6.8 & 1.92 & 24 & 0.049 \\
\hline 4.6 & 6.3 & 2.6 & 23 & 7.0 & 2.00 & 26 & 0.047 \\
\hline 4.8 & 6.5 & 2.7 & 25 & 7.2 & 2.09 & 28 & 0.046 \\
\hline 5.0 & 6.7 & 2.8 & 27 & 7.4 & 2.18 & 29 & 0.044 \\
\hline 5.2 & 6.8 & 2.9 & 28 & 7.6 & 2.27 & 31 & 0.042 \\
\hline 5.4 & 7.0 & 3.0 & 30 & 7.8 & 2.35 & 33 & 0.041 \\
\hline 5.6 & 7.2 & 3.2 & 32 & 7.9 & 2.44 & 35 & 0.040 \\
\hline 5.8 & 7.4 & 3.3 & 34 & 8.1 & 2.53 & 38 & 0.038 \\
\hline 6.0 & 7.5 & 3.4 & 36 & 8.3 & 2.62 & 40 & 0.037 \\
\hline 6.2 & 7.7 & 3.5 & 38 & 8.5 & 2.71 & 42 & 0.036 \\
\hline 6.4 & 7.9 & 3.6 & 40 & 8.7 & 2.79 & 44 & 0.035 \\
\hline 6.6 & 8.1 & 3.7 & 42 & 8.8 & 2.88 & 47 & 0.034 \\
\hline 6.8 & 8.2 & 3.8 & 45 & 9.0 & 2.97 & 49 & 0.033 \\
\hline 7.0 & 8.4 & 3.9 & 47 & 9.2 & 3.06 & 51 & 0.032 \\
\hline
\end{tabular}

